\title{
Nonlinear dynamics of \\ tides and sandbars in tidal channels
}

Tjebbe Hepkema 


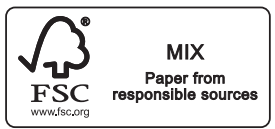

Coastal and Shelf Sea Dynamics group

Institute of Marine and Atmospheric research Utrecht

Department of Physics

Utrecht University

ISBN: 978-94-6416-513-5

Printing: Ridderprint, www.ridderprint.nl

Cover photos: Simone Engelen, www.simone-engelen.com 


\section{Nonlinear dynamics of tides and sandbars in tidal channels}

Niet-lineaire dynamica van getij en zandbanken in getijdengeulen (met een samenvatting in het Nederlands)

Proefschrift

ter verkrijging van de graad van doctor aan de Universiteit Utrecht op gezag van de rector magnificus, prof.dr. H.R.B.M. Kummeling, ingevolge het besluit van het college voor promoties in het openbaar te verdedigen op maandag 12 april 2021 des middags te 2.30 uur

door

\section{Tjebbe Marten Hepkema}

geboren op 21 oktober 1991 te Eelde 
Promotoren: Prof. dr. H.E. de Swart

Prof. dr. H.M. Schuttelaars 
Committee: Prof. dr. GertJan F. van Heijst

Prof. dr. Giovanna Vittori

Prof. dr. Henk A. Dijkstra

Prof. dr. Maarten G. Kleinhans

Dr. Maitane Olabarrieta 



\section{Contents}

1 Introduction 1

1.1 Tides, bars, boats and birds in tidal channels . . . . . . . . 1

1.2 Hydrodynamics in tidal channels . . . . . . . . . . . . . . 2

1.3 Tidal bar dynamics in tidal channels . . . . . . . . . . . . . 3

1.4 Knowledge gaps, objectives and approach . . . . . . . . 8

2 Momentum dissipation on tidal flats 13

2.1 Introduction . . . . . . . . . . . . . . . 15

2.2 Model formulation . . . . . . . . . . . . . . . . . . 16

2.3 Model analysis . . . . . . . . . . . . . . . . . . . . . . . . 19

2.4 Results . . . . . . . . . . . . . . . . . . . . . . . 25

2.5 Discussion . . . . . . . . . . . . . . . . 35

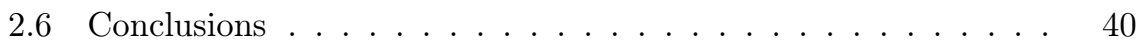

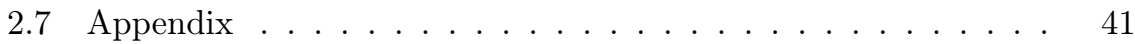

3 Sensitivity of tidal bar wavelength to channel width 45

3.1 Introduction . . . . . . . . . . . . . . . . . 47

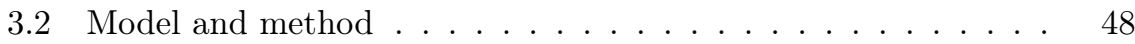

3.3 Results . . . . . . . . . . . . . . . . . . . . . . 54

3.4 Discussion . . . . . . . . . . . . . . . 63

3.5 Conclusions . . . . . . . . . . . . . . . . . 67

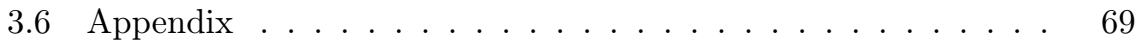

4 Oblique sand ridges in confined tidal channels $\quad 73$

4.1 Introduction . . . . . . . . . . . . . . . . 75

4.2 Model . . . . . . . . . . . . . . . . . . 76

4.3 Results . . . . . . . . . . . . . . . . . . . . . . 81

4.4 Discussion . . . . . . . . . . . . . . . 85

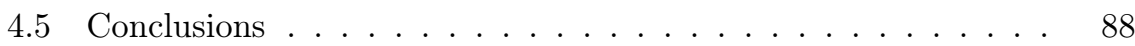


4.6 Appendix . . . . . . . . . . . . . . . . . . 88

5 Local nonlinear tidal bar dynamics $\quad 91$

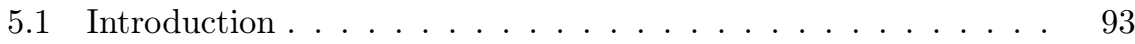

5.2 Methods . . . . . . . . . . . . . . . . . . 95

5.3 Results . . . . . . . . . . . . . . . . . . . . 105

5.4 Discussion . . . . . . . . . . . . . . . . . . . . . 113

5.5 Conclusions . . . . . . . . . . . . . . . . . . . 119

6 Summary and outlook 121

6.1 Summary . . . . . . . . . . . . . . . . . . . . 121

6.2 Outlook . . . . . . . . . . . . . . . . . . 124

$\begin{array}{ll}\text { Bibliography } & 134\end{array}$

$\begin{array}{ll}\text { Nederlandse samenvatting } & 135\end{array}$

$\begin{array}{ll}\text { Acknowledgements } & 139\end{array}$

$\begin{array}{ll}\text { Curriculum vitae } & 141\end{array}$ 


\section{Introduction}

When tidal currents flow over a sandy bottom, the bottom topography of the channel influences the flow. And vice versa: the flow shapes the bottom topography by eroding sediments and depositing them somewhere else. This thesis discusses this nonlinear interaction between the bottom topography and the water motion in straight tidal channels with a length similar to that of the tidal wave.

\subsection{Tides, bars, boats and birds in tidal channels}

All around the globe one finds channels in which the hydrodynamics are driven by tides. Examples are the Western Scheldt in the Netherlands, the Exe Estuary in England, the Ord River Estuary in Australia and channels in the Venice Lagoon in Italy. The bottom of such tidal channels is seldom flat. Often it consists of a combination of bedforms with different length scales: on the centimeter scale one finds ripples, on the decimeter to decameter scale dunes/megaripples and on the hectometer to kilometers scale one finds bars (Dalrymple and Rhodes, 1995, Leuven et al., 2016). In this thesis the focus is on the dynamics of the latter: tidal bars. Examples of these bars are shown in Figure 1.1 and 1.5. These shallow, sometimes intertidal and/or vegetated, areas have been quantitatively described by van Veen (1950), Ahnert (1960) and Dalrymple and Choi (2007). They are important for the local ecosystem (e.g., Bouma et al., 2005) and they protect the coast against extreme water levels during storm surges by dissipating large amounts of wave and tidal energy. Besides the flora and fauna, one often finds intensive marine traffic in tidal channels (see caption Figure 1.2). This is especially true in tidal channels 


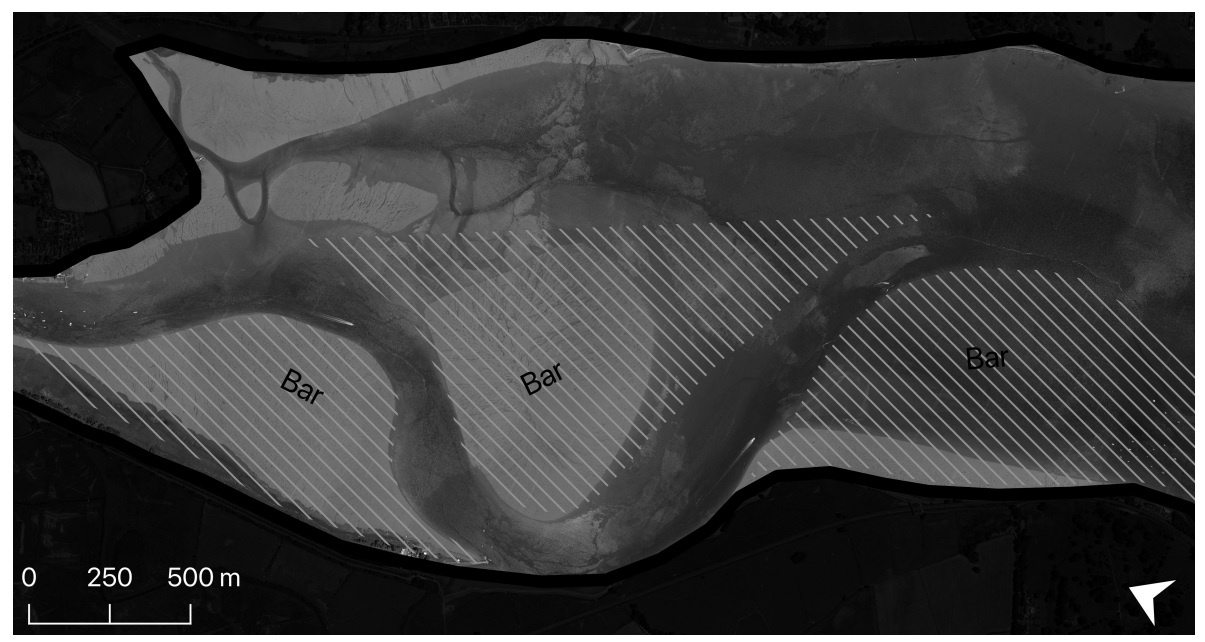

Figure 1.1: Tidal bars (hashed regions) in a tidal channel, Exe Estuary (England). The small white stripes in the deeper, dark areas are ships. Satellite image from Google Earth.

connected to a river (estuaries) with a large harbour. The (cargo)ships that sail trough the channels need to navigate around the tidal bars to avoid grounding (Figure 1.2). To maintain such shipping routes, it is important to understand the formation and evolution of such bars. For example, if one would consider widening the navigation channel to allow more boats to pass, the tidal bar pattern may react to this human intervention. The widening would defeat its purpose if, because of the widening, a new tidal bar pops up in the middle of a navigation channel. Furthermore, sea levels are changing due to the changing climate (IPCC, 2019). This leads to changes in the mean depth, in the tides and also in the bars in the channels (van der Wegen, 2013, Nnafie et al., 2018, Talke and Jay, 2020). Only when one has a solid understanding of how these bars form and how sensitive their dynamics is to changes in the system, proper maintenance of both the ecosystem and the navigation channels is possible.

\section{$1.2 \quad H y d r o d y n a m i c s$ in tidal channels}

Bottom patterns, such as tidal bars, result from convergence of the net (i.e., tidally averaged) transport of sediment. In tidal channels, this sediment is primarily eroded from the bed and transported by tidal currents. So, before one can understand the dynamics of bottom patterns in tidal channels, a thorough understanding of the tides in such channels is needed. One of the simplest models for the hydrodynamics at a certain location in a tidal channel result in sinusoidal functions with the period of the dominant tidal constituent for 


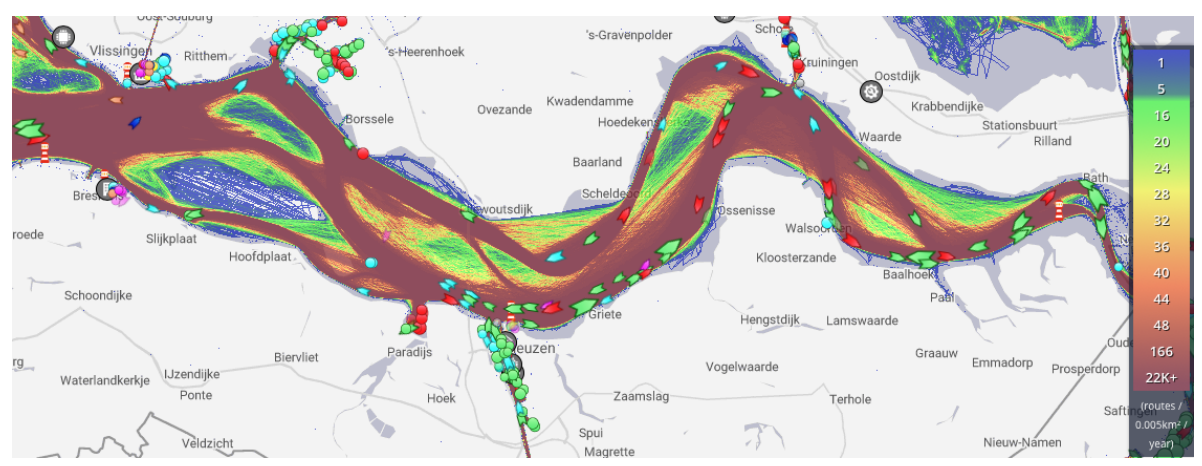

Figure 1.2: Marine traffic in Western Scheldt (the Netherlands) on 6th of October 2020. The colors denote the density of the marine traffic. Areas of low density, away from the coastlines, are tidal bars. Image from https://www.marinetraffic.com.

both the current velocity and the water level (with possibly a phase difference) (e.g., Gerkema, 2019). In many places, for example, in the North Sea and the Wadden Sea (Europe), this period would be half a lunar day. While this simple model gives a first idea about when to expect the high and low waters in a tidal channel or embayment, it fails to explain many other phenomena. For example, the time between high water and low water is not always the same as the time between low and high water (Figure 1.3). Similarly, the strength of maximum ebb current may differ from the strength of the maximum flood current. A simple formulation for sediment transport is the cube (or some other power) of the current velocity (e.g., Bailard, 1981). A distorted sinusoidal tidal curve (i.e., a graph of current speed or water level versus time), may then result in sediment being transported mainly in one direction of the channel. Moreover, spatial variations in, for example, channel depth or width lead to spatial variations in the currents. They in turn cause accumulation of sediments at certain locations in the channel and consequently, bottom patterns form. Hence, the distortions of tidal curves are important for net sediment transport (Aubrey, 1986, Boon and Byrne, 1981, van de Kreeke and Robaczewska, 1993). The distortions of the sinusoidal tidal curves are due to nonlinear hydrodynamic processes (Parker, 1991). Models, which are more complicated than those that yield simple sinusoidal curves, but still mathematically tractable, provide insight in the origin of these distortions (e.g., Friedrichs and Aubrey, 1988, 1994, Ridderinkhof et al., 2014).

\subsection{Tidal bar dynamics in tidal channels}

In the studies mentioned above, the considered timescale (days) is short compared to that of the dynamics of the bottom and hence the bottom evolution 


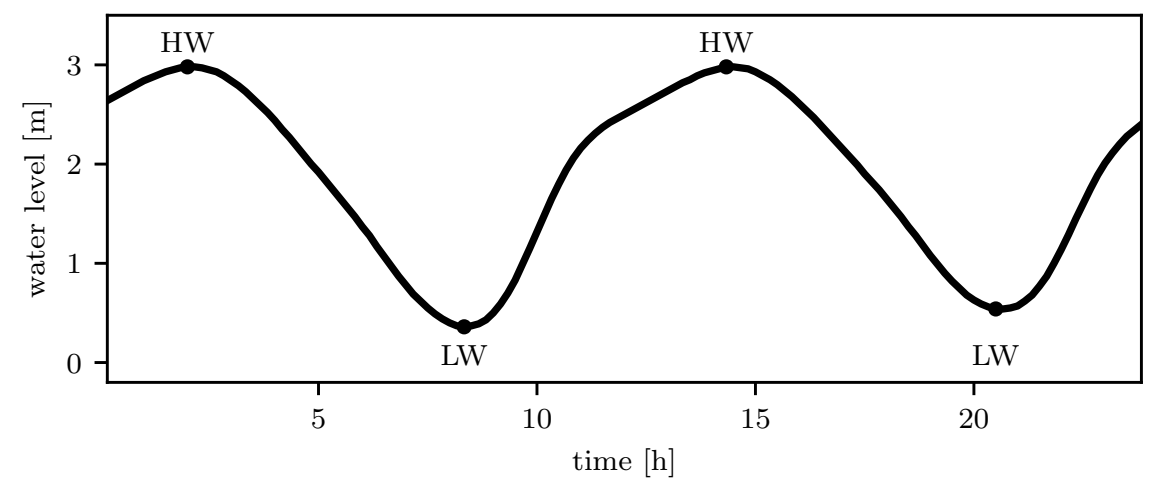

Figure 1.3: Water level with respect to Lowest Astronomical Tide (LAT) versus time at Lauwersoog (the Netherlands) on 21st of October 2020. Data from Rijkswaterstaat (waterinfo.rws.nl).

was not taken into account. On a longer time scale (years to decade) the bottom will evolve. Seminara and Tubino (2001) developed a model consisting of the $3 \mathrm{D}$ shallow water equations coupled with sediment transport equations and an equation for the evolution of the bottom. Their model is a so-called local model, meaning that they consider only a short section of long channel with periodic (along-channel) boundary conditions. The section is short compared to the tidal wavelength and the length scale on which variations in the channel width occur. The channel itself is long such that at certain locations the effect of the (along-channel) boundaries can be neglected. These assumptions have two important advantages. The first is that the patterns are studied in absence of the effects by closed landward or open seaward boundaries. Secondly, in such a model set-up, a horizontally flat bottom with a spatially uniform tidal current flowing over it can be shown to be a morphodynamic equilibrium. That is, it is a bottom pattern that does not change over time. And since the bottom height changes at locations where net sediment transport converges, this implies that in an equilibrium state, the net sediment transport convergence vanishes.

However, a morphodynamic equilibrium is not necessarily stable. This can be understood as follows. When a spatially uniform tidal current flows over a flat sandy bed and either the hydrodynamics or the bottom height (or both) are perturbed, the system may enter a positive feedback loop: the perturbations grow. Seminara and Tubino (2001) demonstrated that the initial formation of tidal bars may be interpreted as the result of such a positive feedback loop. They showed that, of all the possible perturbations of the flat bottom, the ones that grow the fastest, form tidal bar patterns with wavelengths that roughly correspond to observed wavelengths in the field. Later, Schramkowski et al. (2002) and Garotta et al. (2006) showed that this is also possible in 


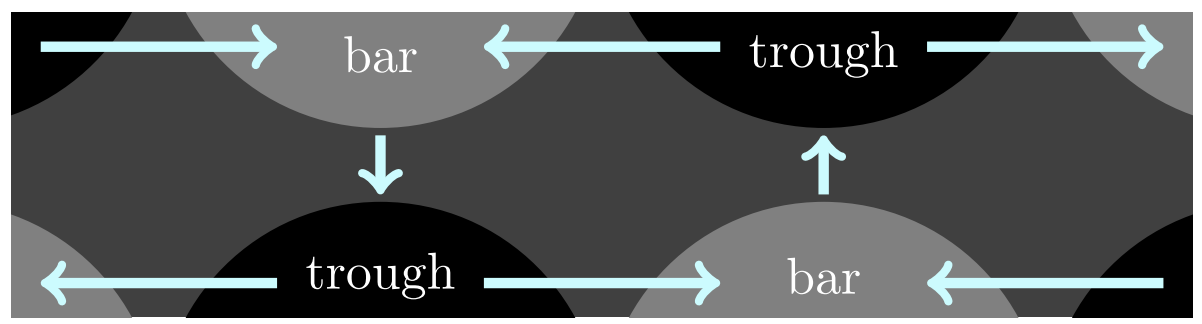

Figure 1.4: Residual currents (arrows) induced by alternating tidal bars, as found with a local model that considers a stretch of a tidal channel that is short compared to its total length. Note the convergence of residual along-channel currents above the bars.

a depth-averaged model and that the three spatial dimensions considered by Seminara and Tubino (2001) are not needed. Moreover, Schramkowski et al. (2002) added the local inertial term in the momentum balance, which becomes relevant when the channel width is of the same order of magnitude as the tidal excursion length and friction does not dominate the flow. Garotta et al. (2006) investigated the formation of tidal bars when the current is not forced by a perfectly sinusoidal pressure gradient force (as was done by Seminara and Tubino (2001) and Schramkowski et al. (2002)). Instead, they consider a distorted one, consisting of both a principle frequency and its first overtide. This asymmetric forcing resulted in migrating tidal bars when the maximum current in one direction was stronger than in the other direction. Together, these studies revealed two key factors for the formation of tidal bars: depth-dependent bottom friction and the advection of vorticity. Zimmerman (1981) already explained how (in a variety of systems) the joint appearance of these factors results in the generation of a residual circulation to which tidal bars owe their existence (Figure 1.4).

The studies above discuss the 'initial' formation of tidal bars. The word 'initial' is a bit ambiguous, as it assumes that first there was a channel with a flat bottom and then the bars emerged. However, it does explain why a certain equilibrium, the flat bottom with an spatially uniform current flowing over it, can be unstable. And since the fastest growing bottom perturbation resembles to a certain extent observed patterns, studying the stability of this rather trivial equilibrium was insightful. Moreover, these models are fast: the fastest growing bottom perturbation is calculated in a fraction of seconds. This allows for extensive sensitivity studies of model results to changes in the values of model parameters. A glance at a book filled with sediment transport formulations (Soulsby, 1997), reveals that the answer to the question how does sediment transport depends on tidal current, is not indefinitely answered. This, in combination with the numerous simplifications and assumptions made in the studies mentioned above, shows that sensitivity analyses are important to make 
sure that the conclusions do not disappear in the uncertainty. A major limitation of models that are restricted to initial formation of bars is that they assume the height of these bars to be very small with respect to water depth. However, natural bars do not obey this condition.

Moreover, there are reasons to believe that, besides the flat bottom with a spatially uniform tidal current flowing over it, other (less trivial) bottom and flow patterns that result in a vanishing bottom evolution exist. These equilibria are searched for and studied with numerical models that do not have such restrictions on the bar height and they provide a global, rather than a local description of the bars/tidal channel/system (Hibma et al., 2003, 2004, van der Wegen and Roelvink, 2008, Tambroni et al., 2010, van der Wegen and Roelvink, 2012, van der Wegen, 2013, Dam et al., 2016, Xie et al., 2017, Nnafie et al., 2018, Olabarrieta et al., 2018). Their domains represent an entire channel from an open boundary at the seaward side up to a closed landward boundary (or river), with imposed tidal motion at the open boundary. Subsequently, a numerical time integration (over centuries) is performed until the bottom patterns hardly change. The latter correspond to states that are (potentially close to) nontrivial equilibria (i.e., equilibria different from the flat bottom). When choosing a realistic channel geometry, these numerical models simulate bottom patterns that compare reasonably well with observed bottom patterns (Figure 1.5). However, their complexity hinders a detailed analysis of the dynamics, since many processes happen at the same time. In particular, due their complexity and included processes that become important at long time scales, the 'equilibria' found by the complex models are often still evolving, albeit slowly. Moreover, there is no guarantee that if one were to continue the simulation for a longer time, the pattern would not change in a possibly completely different pattern.

Complementary to the models described above, tidal bars were also studied in a laboratory by Tambroni et al. (2005) and Leuven and Kleinhans (2019). Tambroni et al. (2005) report that the tidal bars that they simulated in their laboratory have characteristics that are consistent with those predicted by the theoretical model of Seminara and Tubino (2001), although the tidal bar wavelength was slightly smaller than that expected from the latter study. The channel of Tambroni et al. (2005) had one open end and one closed end. In contrast, the set-up of Leuven and Kleinhans (2019) was as close as possible to a local model, by using a periodically tilting flume with two open sides. Besides a correspondence with the theory of Schramkowski et al. (2002) during the initial formation of the bars, they reproduced the formation of so-called sills. The latter are narrow straight 'bridges' between the alternating tidal bars. Moreover, Leuven and Kleinhans (2019) attributed the differences between the initial formation and the long-term behavior to nonlinear processes and highlighted the need of a further development of the theory of nonlinear tidal bar dynamics to explain their observations.

There is one modelling study of nontrivial equilibria in a local model: 

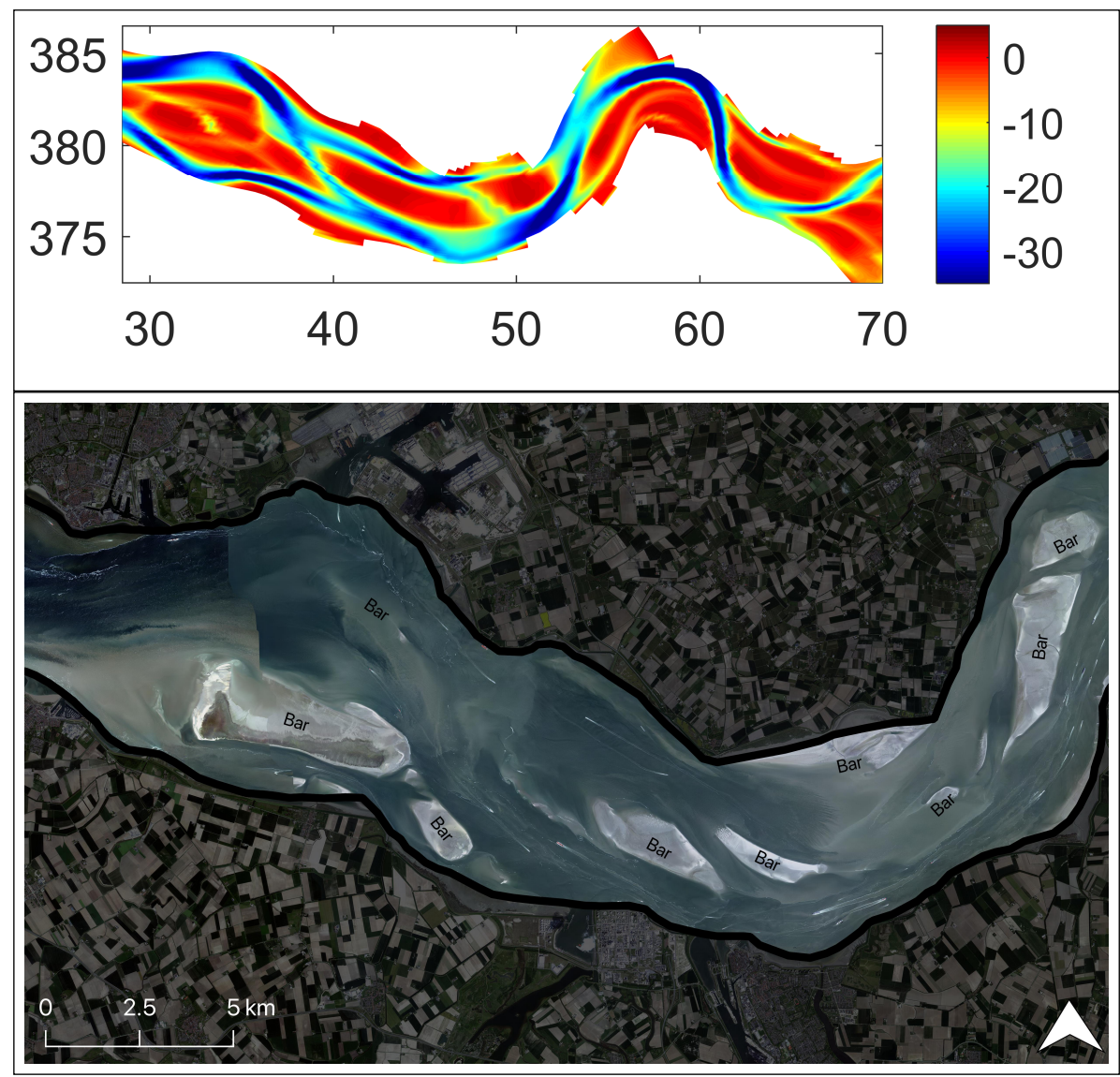

Figure 1.5: Top panel: simulated bottom height by the complex numerical model of Nnafie et al. (2018). The colors denote the channel depth in meters, the numbers on the horizontal and vertical axes represent distance in kilometers. Bottom panel: satellite image of tidal bars in the Western Scheldt (the Netherlands). Note the correlations between areas where tidal bars occur and areas of low traffic density in Figure 1.2. Satellite image from: NSO Satellietdataportaal (C)21AT 2017, distribution 21AT, all rights reserved, https://satellietdataportaal.nl. 
Schramkowski et al. (2004). They studied the equilibria and stability of mature tidal bars by projecting the governing equations on Fourier modes and performing a bifurcation analysis with the channel width and the friction parameter as bifurcation parameters. Besides the simplicity due to the local nature of the model, their bifurcation analysis poses several advantages over the numerical models discussed above. In contrast to the numerical models that employ a time integration methodology, Schramkowski et al. (2004) used a Newton method to find stationary bottom patterns. Their model allowed them to explicitly calculate the stability of the morphodynamic equilibria. That is, they performed a linear stability analysis of the equilibria. Moreover, in contrast to time integration methods, their methodology allows one to find unstable equilibria, which are helpful in understanding the dynamics (of which the unstable flat bottom is the proof). The resulting dynamical structure is rich: multiple equilibria for the same parameter values and periodic solutions. However, their model was limited to weak friction. To calculate equilibria with (realistically) large friction parameters, many (but unclear which) Fourier modes were needed. This made the continuation to realistic values of bottom friction unfeasible.

\subsection{Knowledge gaps, objectives and approach}

The above motivates the main goal of this thesis: contribute to a deeper understanding of the dynamics of tides and tidal bars in tidal channels. This goal is met through four specific objectives. The first concerns the tides in a channel, the second and third concerns the initial formation of tidal bars and the fourth is about the long-term tidal bar dynamics.

\subsubsection{Gap, objective and approach 1}

Section 1.2 concluded that knowledge of nonlinear hydrodynamical processes in tidal channels is needed. One of the nonlinear hydrodynamical process that is not yet well understood is the momentum dissipation on tidal flats. When the water level rises in a tidal channel consisting of a deep main channel and shallow adjacent tidal flats, water flows from the channel onto the adjacent tidal flats, carrying with it longitudinal momentum. The mass is temporarily stored on the flats, until it flows back into the channel during the falling tide. The momentum, on the other hand, dissipates rapidly due to high friction on the tidal flats. That is, the flats act like mass storages and momentum sinks (Dronkers and Schönfeld, 1959, Dronkers, 1964, Speer, 1984). The effect of the mass storage on tidal characteristics, such as ebb-flood asymmetry, has been investigated before (Speer and Aubrey, 1985, Friedrichs and Aubrey, 1994, Ridderinkhof et al., 2014). However, the effect of the dissipation of momentum on the tidal flats is less well understood. This is the first objective: 
1. Understand and quantify the sensitivity of tidal characteristics (such as times of high and low water, tidal range, ratio between maximum flood and ebb currents, etc.) to momentum dissipation on tidal flats.

This will be done by extending the cross-sectionally averaged shallow water model of Speer and Aubrey (1985) to include the effects of the momentum sink. Moreover, their model domain is generalized to a double inlet (i.e., a channel that is open on two sides) rather than a semi-enclosed basin. In the double inlet system the hydrodynamics are forced by the tides on both open boundaries. An example of such an inlet is the Marsdiep-Vlie system in the Wadden Sea. This model domain is a generalization of the one in Speer and Aubrey (1985) in the sense that, when the tides at the two open boundaries are identical, the dynamics in half the double inlet reduce to that in a semienclosed basin. The model describes the hydrodynamics in a long tidal channel. So, in contrast to the local morphodynamical models discussed earlier, here the 'global' dynamics is modelled and the bottom topography is fixed. The parameter regime considered is that where the Froude number, which measures the degree of nonlinearities in the system, is small $(\approx 0.1)$. By exploiting this small parameter, a perturbation analysis provides a semi-analytical investigation of the effect of the momentum sink on the tidal characteristics.

\subsubsection{Gap, objective and approach 2}

When analyzing the characteristics of tidal bars in the field and in laboratory settings, the (wave)length of tidal bars seems to correlate with the channel width (Dalrymple and Rhodes, 1995, Leuven et al., 2016). Moreover, results obtained with numerical studies (e.g., Hibma et al., 2004, van der Wegen and Roelvink, 2008, van de Lageweg and Feldman, 2018) revealed that the tidal bar wavelength depends on the current velocity, channel depth and the channel width. However, in the local model of Schramkowski et al. (2002), the modelled tidal bar wavelength hardly depends on the channel width. Since this model was fruitful in unraveling the mechanism of the initial tidal bar growth, it is worth investigating what causes the inconsistency between Schramkowski et al. (2002) on one hand and the observations and numerical studies on the other hand. Solving this inconsistency is the second objective:

2. Extend the model of Schramkowski et al. (2002) such that the tidal bar wavelength does depend on the channel width, while still roughly reproducing natural (observed) tidal bar patterns, and understand what crucial physics is added to resolve this inconsistency.

The approach is to extend the model of Schramkowski et al. (2002) by including the effects of turbulent exchange of momentum and sediment in the horizontal plane. This is motivated by the fact that these terms are present in numerical models, while they are neglected in Schramkowski et al. (2002). Moreover, to 
ensure the conclusions are trustworthy and to gain additional insight in the sensitivity of the tidal bar wavelength to the model parameters, a sensitivity analysis is performed to the erosion parameter, background current velocity amplitude, channel depth, channel width, bed slope effect, settling velocity, horizontal eddy diffusivity and viscosity and the critical velocity for erosion.

\subsubsection{Gap, objective and approach 3}

Seminara and Tubino (2001) considered relatively narrow tidal channels (few 100 meters wide) with currents of approximately $1 \mathrm{~m} \mathrm{~s}^{-1}$ and at latitudes of $45^{\circ} \mathrm{N}$, such as in the Venice Lagoon. These parameters result in a so-called Rossby number much larger than one. As a result, the Coriolis effect is negligible. Schramkowski et al. (2002) considered wider channels and based their model set-up on the Western Scheldt. This channel is at $51^{\circ} \mathrm{N}$, has a width of approximately $5 \mathrm{~km}$, a depth of $10 \mathrm{~m}$ and currents with amplitudes of $1 \mathrm{~m} \mathrm{~s}^{-1}$. In contrast to the parameter values representative for the tidal channels in the Venice Lagoon, result these values in a Rossby number close to one. That is, channel is not narrow anymore: the the dynamics are significantly influenced by the Coriolis effect. So, even though the simulated patterns of Schramkowski et al. (2002) resembled the observed patterns reasonably well, it is necessary to retain the Coriolis effect. Or, at least one needs to be sure its effect is minimal before neglecting it. In fact, the Coriolis effect has been shown to be relevant for the hydro-morphodynamics in certain tidal channels (Valle-Levinson, 2008, Winant, 2008, Xie et al., 2017, Olabarrieta et al., 2018). However, the role of the Coriolis effect on the initial formation of tidal bars was never investigated. This is the third objective:

3. Study the role of the Coriolis effect on the initial formation of bottom patterns in a tidal channel.

The specific interest is in the spatial pattern and growth rate of the fastest growing bottom pattern. After the analysis concerning the second objective, it turned out that the sediment transport formula used in that chapter can be further simplified. Using those simplifications, but adding the Coriolis effect, the linear stability analysis is repeated to meet the third objective.

\subsubsection{Gap, objective and approach 4}

As stated above, when tidal bars mature and their heights are no longer small compared to the water depth, their dynamics become nonlinear. The long-term evolution of tidal bars is studied in laboratory settings and in numerical models. Only one of these numerical models is a local model, which was bound to consider weak friction. To bridge the gap between the local models that study the initial formation, the local model that is bound to weak friction and the global numerical models that study the long-term dynamics, a local model that 
systematically finds nontrivial tidal bar equilibria in physically relevant parameter regimes is needed. Field observations suggest that the channel width is an important parameter for the tidal bar dynamics, a finding that is also observed in the available local models and complex numerical models (see earlier citations). Moreover, numerical studies (Xie et al., 2017, Olabarrieta et al., 2018) show that there are physically relevant parameter regimes where the Coriolis force significantly affects the initial growth of the spatial patterns of tidal bars. The fourth objective is therefore:

4. Investigate the long-term dynamics of tidal bars, including the possible presence of nontrivial morphodynamic equilibria in a local model, compare the long-term dynamics with the initial formation and assess the sensitivity of the results to the Coriolis parameter and channel width.

This is done by developing a model for bars in a tidal channel, which is a modification of that of Yuan et al. (2016) to study tidal sand ridges on the continental shelf. The new local model has the option to integrate in time, such as the other (non local) numerical models. This allows one to study the transient behavior of the tidal bars and compare this with the evolution of tidal bars in a laboratory described by Leuven and Kleinhans (2019). Moreover, by using a root-finding algorithm, the model finds morphodynamic equilibria and their stability directly.

\subsubsection{Overview of the following chapters}

Chapters $2-5$ focus on the objectives $1-4$ of this thesis, respectively. The final Chapter 6 presents a summary and outlook. 
Chapter 1. Introduction 


\section{Momentum dissipation on tidal flats}

T. M. Hepkema, H. E. de Swart, A. Zagaris and M. Duran-Matute.

Sensitivity of tidal characteristics in double inlet systems to momentum dissipation on tidal flats: a perturbation analysis. Ocean Dynamics 68, 439-455 (2018). https://doi.org/10. $1007 /$ s10236-018-1142-z 


\begin{abstract}
In a tidal channel with adjacent tidal flats along-channel momentum is dissipated on the flats during rising tides. This leads to a sink of along-channel momentum. Using a perturbative method, it is shown that the momentum sink slightly reduces the $\mathrm{M}_{2}$ amplitude of both the sea surface elevation and current velocity and favours flood dominant tides. These changes in tidal characteristics (phase and amplitude of sea surface elevations and currents) are noticeable if widths of tidal flats are at least of the same order as the channel width, and amplitudes and gradients of along-channel velocity are large. The $\mathrm{M}_{2}$ amplitudes are reduced because stagnant water flows from the flats into the channel, thereby slowing down the current. The $\mathrm{M}_{4}$ amplitudes and phases change because the momentum sink acts as an advective term during the fall of the tide, such a term generates flood dominant currents. For a prototype embayment that resembles the Marsdiep-Vlie double-inlet system of the Western Wadden Sea, it is found that for both the sea surface elevation and current velocity, including the momentum sink leads to a decrease of approximately $2 \%$ in $\mathrm{M}_{2}$ amplitudes and an increase of approximately $25 \%$ in $\mathrm{M}_{4}$ amplitudes. As a result, the net import of coarse sediment is increased by approximately $35 \%$, while the transport of fine sediment is hardly influenced by the momentum sink. The impact of the momentum sink on tidal characteristics becomes larger with increasing ratio of intertidal area and channel area, and is in a much lesser extent sensitive to the slope of the flats, drag coefficient, embayment length and the phase difference between incoming tidal waves at the boundary of the embayment. For the Marsdiep-Vlie system, the $\mathrm{M}_{2}$ sea surface amplitude obtained from the idealized model is similar to that computed with a realistic three-dimensional numerical model whilst the comparison with regard to $\mathrm{M}_{4}$ improves if momentum sink is accounted for.
\end{abstract}




\section{$2.1 \quad$ Introduction}

In shallow coastal seas, such as the Wadden Sea in Northern Europe, the tidal motion is highly nonlinear, as is evident from the presence of residual and higher harmonics in the primary tide (Buijsman and Ridderinkhof, 2007). Well-known sources of these nonlinear tides are the advection of momentum, quadratic bottom stress, depth dependent friction, divergence of excess mass (i.e. mass stored between mean sea level and actual sea surface) and the hypsometry of the embayment (e.g. Parker, 1991, Friedrichs and Aubrey, 1994, and references therein). As a result, the sinusoidal shape of the curves of the sea surface height and current velocity at a fixed location is distorted. This tidal asymmetry leads to a net transport of fine and coarse sediment (Groen, 1967, Aubrey, 1986, Boon and Byrne, 1981, van de Kreeke and Robaczewska, 1993).

In this study, the focus is on nonlinear tides by hypsometry and in particular by dissipation of momentum on tidal flats. When the water level rises, water flows from the channel onto the flats, carrying with it longitudinal momentum. The mass is temporarily stored on the flats, until it flows back into the channel during the falling tide. The momentum, on the other hand, dissipates rapidly due to high friction on the tidal flats. That is, the flats act like mass storages and momentum sinks (Dronkers and Schönfeld, 1959, Dronkers, 1964).

In contrast to mass storage, which has been extensively studied (Speer and Aubrey, 1985, Friedrichs and Aubrey, 1994, Ridderinkhof et al., 2014), the role of the momentum sink on tidal dynamics is less well understood. Numerical model studies (e.g. Brown and Davies, 2010) show that tidal asymmetry strongly depends on the distribution of channels and flats, thereby suggesting the importance of processes such as the momentum sink. De Swart et al. (2011) and Alebregtse (2015) studied numerically the momentum sink in a semi-enclosed embayment. They demonstrated that the amount by which the momentum sink deforms tidal curves depends on the ratio of flat-to-channel area, the shape of the tidal flats and the distribution of flats along the channel. However, no details about the underlying processes were given.

The considerations above motivate further investigation of the generation of overtides by a momentum sink, which is conducted here. Similar to Alebregtse (2015), the model of Speer and Aubrey (1985) is extended with a momentum sink term. The model of Speer and Aubrey (1985) has been successfully validated against field data (see also Friedrichs and Aubrey, 1988, 1994). Here, two main new aspects are introduced. First, the approach is analytic in nature, and approximate solutions of the cross-sectionally averaged shallow water equations are explicitly constructed using perturbation methods that exploit the smallness of the Froude number. This enables the assessment of the impact of the momentum sink and quantification of the difference between solutions that do and do not account for it. Second, a double inlet system (rather than a semi-enclosed bay) with an $\mathrm{M}_{2}$ tidal wave entering at both ends is considered. 
In particular, this permits investigating what happens in double inlet systems when the two waves entering at opposite sides differ in amplitude or phase, as typically occurs in nature. Examples of such embayments are the Western Wadden Sea in the Netherlands, with the Marsdiep and Vlie inlets (e.g. Ridderinkhof, 1988, also considered here), the Laguna de Términos in México (David and Kjerfve, 1998) and the Santa María La Reforma in California (Serrano et al., 2013).

This study has two specific aims. The first is to assess changes in tidal characteristics of sea surface elevation and current velocity due to the momentum sink in double inlet systems with different parameter settings. The parameters that will be varied are the width of the tidal flats and the channel width, the drag coefficient, the slope of the flats, the phase difference between the incoming tidal waves and the length of the embayment. The second aim is to understand the mechanisms behind these changes in tidal characteristics.

This chapter is organized as follows. In Section 2.2, the model is presented. In Section 2.3, a perturbation method is used to find approximate solutions to the cross-sectionally averaged shallow water equations. The results are presented in Section 2.4, followed by a discussion in Section 2.5. Here, mechanisms leading to the model results are explained together with a qualitative comparison with the model output of a complex numerical model. Section 2.6 contains the conclusions.

\subsection{Model formulation}

\subsubsection{Physical domain and geometry}

The tidal embayment considered in this study consists of a channel of constant width $b_{c}^{*}$ with adjacent tidal flats. The embayment is connected to an open sea on both sides, has length $L^{*}$ and geometry symmetric with respect to the centerline of the channel (see figure 2.1).

The along-channel coordinate is $x^{*}$, the lateral coordinate $y^{*}$, the vertical coordinate $z^{*}$ and the time coordinate $t^{*}$. The origin is placed in the middle of the embayment at the height of the undisturbed sea level. The undisturbed water depth on the flats is $d_{f}^{*}$ and in the channel it is $h_{c}^{*}$. The width of the channel or of the flats henceforth refers to the width of the wetted part of the channel or flats. The total width $b^{*}$ of the embayment is dependent on the sea surface elevation $\zeta^{*}$, and hence also on $t^{*}$ and $x^{*}$. The geometry of the cross section is based on that of Speer and Aubrey (1985). It is assumed that the lowest part of the flats are located at the level of low water. The total width reads

$$
b^{*}=b_{\max }^{*}-\frac{2 d_{f}^{*}}{\tan \varphi}\left(1-\frac{\zeta^{*}}{d_{f}^{*}}\right),
$$



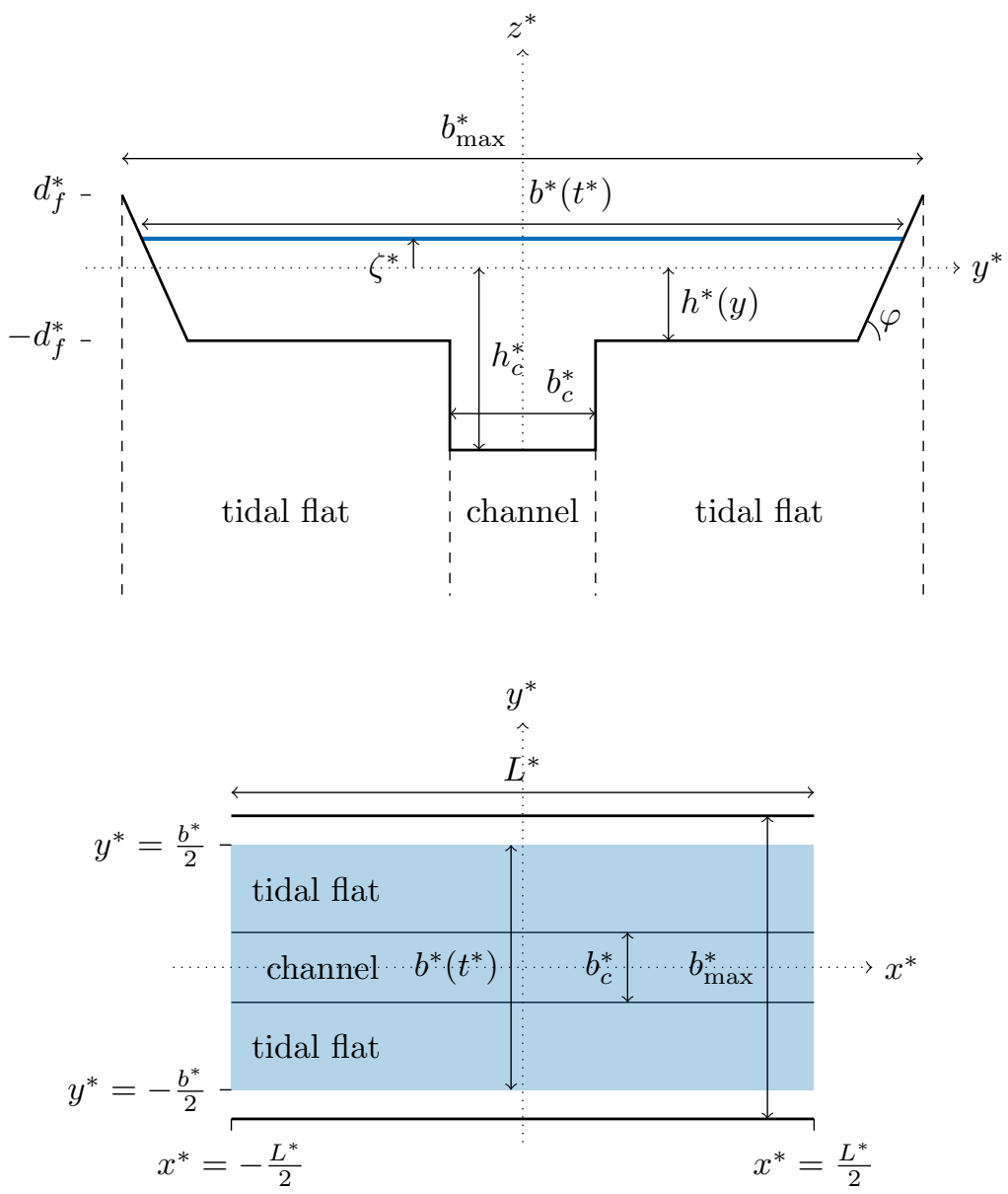

Figure 2.1: Geometry of the open embayment (a: side view at any longitudinal position, b: top view). Here, $b_{\max }^{*}$ is the width of the embayment including the dry parts of the flats, $b^{*}\left(t^{*}\right)$ the width of the wetted part of the embayment and $b_{c}^{*}$ the width of the channel. Furthermore, $h^{*}$ is the undisturbed depth (being $h_{c}^{*}$ in the channel and $d_{f}^{*}$ on the flats), $\varphi$ the angle of inclination of the flats, $L^{*}$ the length of the embayment and $\zeta^{*}$ the sea surface elevation. The origin is at the intersection of the dotted lines. In (a), the $x^{*}$ axis points into the paper. The blue area in (b) is the wetted part. 
where $d_{f}^{*}$ is the water depth on the flats, $b_{\max }^{*}$ is the width of the embayment including the dry parts of the flats and $\varphi$ the angle of inclination of the flats as in figure 2.1. It follows from the figure that partly horizontal flats with a kink are possible if

$$
\varphi \geq \tan ^{-1}\left(\frac{4 d_{f}^{*}}{b_{\max }^{*}-b_{c}^{*}}\right) .
$$

This motivates the introduction of a parameter $q \geq 1$ defined as

$$
q=\tan (\varphi) \frac{b_{\max }^{*}-b_{c}^{*}}{4 d_{f}^{*}} .
$$

The total width of the embayment now reads

$$
b^{*}=b_{\max }^{*}-\frac{b_{\max }^{*}-b_{c}^{*}}{2 q}\left(1-\frac{\zeta^{*}}{d_{f}^{*}}\right) .
$$

The parameter $q \geq 1$ determines flat steepness; $q=1$ means linear flats and larger $q$-values imply steeper flats (in figure $2.1, q=5$ ).

\subsubsection{Governing equations}

The hydrodynamics in the embayment are modelled by the cross-sectionally averaged shallow water equations. They read

$$
\begin{aligned}
& \frac{b^{*}}{b_{c}^{*}} \frac{\partial \zeta^{*}}{\partial t^{*}}+\frac{\partial\left(\zeta^{*}+h_{c}^{*}\right) u^{*}}{\partial x^{*}}=0 \\
& \frac{\partial u^{*}}{\partial t^{*}}+u^{*} \frac{\partial u^{*}}{\partial x^{*}}+g^{*} \frac{\partial \zeta^{*}}{\partial x^{*}}+\frac{r^{*} u^{*}}{\zeta^{*}+h_{c}^{*}}=M^{*}
\end{aligned}
$$

with

$$
M^{*}=\frac{b^{*}-b_{c}^{*}}{b_{c}^{*}} \frac{u^{*}}{\zeta^{*}+h_{c}^{*}} \frac{\partial \zeta^{*}}{\partial t^{*}} \mathcal{H}\left(-\frac{\partial \zeta^{*}}{\partial t^{*}}\right) .
$$

Here, $u^{*}\left(\mathrm{~m} \mathrm{~s}^{-1}\right)$ is the current velocity averaged over the cross-section of the channel (excluding the flats) in the along-channel $\left(x^{*}\right)$ direction, $\zeta^{*}(\mathrm{~m})$ is the sea surface elevation, $g^{*}\left(\mathrm{~m} \mathrm{~s}^{-2}\right)$ is the gravitational acceleration, $r^{*}\left(\mathrm{~m} \mathrm{~s}^{-1}\right)$ is a friction parameter and $\mathcal{H}$ is the step or Heaviside function, which is one (zero) when its argument is positive (negative). Further, $M^{*}$ is the momentum sink term which is derived and put into context of previous studies in Appendix 2.7.1.

In equations (2.2.3)-(2.2.5), it is assumed that the channel width $b_{c}^{*}$ is constant, the water in the channel has a constant density, there is no effect of wind or waves and the stresses that result from averaging nonlinear momentum fluxes over the cross section can be ignored. On the tidal flats, the velocity in the along-channel direction is assumed to be zero. Instead of a quadratic bottom 
stress $\tau_{b}^{*}=\rho^{*} c_{d}\left|u^{*}\right| u^{*}$, with $c_{d}$ a drag coefficient and $\rho^{*}$ the water density, a linear stress $\tau_{b}^{*}=\rho^{*} r^{*} u^{*}$ is used to allow for analytical approximations. The friction parameter $r^{*}$ is determined by demanding that the energy dissipated in one tidal cycle (averaged over the spatial domain) is to be the same in both formulations (e.g. Lorentz, 1922, Terra et al., 2005). Thus,

$$
\int_{-L^{*} / 2}^{L^{*} / 2}\left\langle c_{d}\left|u^{*}\right| u^{* 2}\right\rangle d x^{*}=\int_{-L^{*} / 2}^{L^{*} / 2}\left\langle r^{*} u^{* 2}\right\rangle d x^{*},
$$

in which $\langle\cdot\rangle$ denotes the average over one tidal cycle. As, a priori, $u^{*}$ is not known, $r^{*}$ is determined iteratively.

The system of equations (2.2.1)-(2.2.5) will be considered in the weakly nonlinear regime, i.e. magnitudes of nonlinear terms are small compared to those of linear terms. In that case, as will be shown in Section 2.3, solutions consist of waves that propagate in both the positive and negative $x$-direction. The boundary conditions are that at both open sides of the embayment, a prescribed $T^{*}$-periodic tidal wave (where $T^{*}=2 \pi / \sigma^{*}$, with $\sigma^{*}$ the $\mathrm{M}_{2}$ tidal frequency) propagates into the embayment and that waves may freely exit the domain. This choice implies that boundary conditions are independent of the dynamics inside the domain.

Furthermore, it is imposed that the average volume of water over one tidal period is constant and that there is no net transport of water through the embayment. The former poses a condition for the residual sea surface elevation and the latter a condition for the residual current velocity.

\subsection{Model analysis}

Equations (2.2.3)-(2.2.5) are nonlinear and cannot be solved analytically, necessitating the derivation of approximate solutions, either numerical or perturbative. The former have the advantage that they remain valid over extended regions in parameter space, as they do not require nonlinear terms to be much smaller than the linear ones. The benefit of perturbative methods is that they result in closed form solutions that yield information about the effect of different physical forcing terms on solutions. As mentioned in the Introduction, here a perturbation analysis is made. For this, dimensionless equations containing a small parameter are needed in which all variables are of order 1 . Let

$$
u=\frac{u^{*}}{\mathcal{U}^{*}}, \quad \zeta=\frac{\zeta^{*}}{\mathcal{Z}^{*}}, x=\frac{x^{*}}{\mathcal{L}^{*}} \text { and } t=\frac{t^{*}}{\mathcal{T}^{*}}
$$

be dimensionless variables, where

$$
\mathcal{U}^{*}=\mathcal{Z}^{*} \sqrt{\frac{g^{*}}{h_{c}^{*}}}, \quad \mathcal{Z}^{*}=d_{f}^{*}, \quad \mathcal{L}^{*}=\frac{\sqrt{g^{*} h_{c}^{*}}}{\sigma^{*}} \text { and } \mathcal{T}^{*}=\frac{1}{\sigma^{*}}
$$


The scales $\mathcal{U}^{*}$ and $\mathcal{Z}^{*}$ are typical values for the current velocity and the sea surface elevation amplitude, respectively. The scales $\mathcal{L}^{*}$ and $\mathcal{T}^{*}$ are typical values for a frictionless tidal wave length and period of the principal constituent (both divided by $2 \pi$ ), quantifying the length and time scale of the dominant dynamics. The scaled versions of equations (2.2.3)-(2.2.4) are

$$
\begin{array}{r}
(1+\beta-\beta \alpha \varepsilon(1-\zeta)) \frac{\partial \zeta}{\partial t}+\frac{\partial}{\partial x}[(1+\varepsilon \zeta) u]=0, \\
\frac{\partial u}{\partial t}+\varepsilon u \frac{\partial u}{\partial x}+\frac{\partial \zeta}{\partial x}+\frac{r u}{1+\varepsilon \zeta}-\varepsilon \beta \frac{u}{1+\varepsilon \zeta} \frac{\partial \zeta}{\partial t} \mathcal{H}\left(-\frac{\partial \zeta}{\partial t}\right)=0 .
\end{array}
$$

The Froude number is denoted by $\varepsilon$ :

$$
\varepsilon=\frac{\mathcal{Z}^{*}}{h_{c}^{*}}=\frac{\mathcal{U}^{*}}{\sqrt{g^{*} h_{c}^{*}}} .
$$

In many tidal embayments (e.g., the Wadden Sea), $\varepsilon$ is around $10^{-1}$ and here assumed to be a small parameter. The dimensionless length of the channel is $L=L^{*} / \mathcal{L}^{*}$. Furthermore,

$$
\beta=\frac{b_{\max }^{*}-b_{c}^{*}}{b_{c}^{*}}, \alpha \varepsilon=\frac{1}{2 q}, \text { and } r=\frac{r^{*} \mathcal{T}^{*}}{h_{c}^{*}} .
$$

The parameter $\beta$ is the ratio of the tidal flat width to the main channel width, the parameter $\alpha$ controls the steepness of the sides of the flats and $r$ is the dimensionless friction parameter. It is assumed that the area of the flats is of the same order as the channel area, that the slope of the tidal flats is steep and that friction is moderate. The consequence of these choices are that the magnitude of the momentum sink and mass storage are comparable with that of other nonlinear terms in the equations of motion, such as advection. Note that Friedrichs and Aubrey (1994) and Speer and Aubrey (1985) assume $\beta$ to be small. However, inlet systems like those in the Wadden Sea are characterized by $\beta \approx 2$ (Dronkers, 2005). Thus, it is assumed that

$$
\beta, \alpha, r=O(1)
$$

where the symbol $O(1)$ denotes that they are of asymptotic order 1 . Moreover, $O(\varepsilon)$ parts of the friction parameter $r$ and the $O\left(\varepsilon^{2}\right)$ parts of the last term in equation (2.3.4) are neglected. All dimensionless variables are assumed to be of order 1 , so the magnitude of the terms in the dimensionless equations is determined by the parameters they involve.

The Lorentz linearization condition (2.2.6) in dimensionless variables reads

$$
\frac{\mathcal{U}^{*} h_{c}^{*} c_{d}}{\mathcal{T}^{*}} \int_{-L / 2}^{L / 2}\left\langle|u| u^{2}\right\rangle d x=r \int_{-L / 2}^{L / 2}\left\langle u^{2}\right\rangle d x
$$


with the additional assumption that

$$
\frac{\mathcal{U}^{*} h_{c}^{*} c_{d}}{\mathcal{T}^{*}}=O(1) .
$$

Substituting the perturbation series,

$$
u=u_{0}+\varepsilon u_{1}+O\left(\varepsilon^{2}\right) \text { and } \zeta=\zeta_{0}+\varepsilon \zeta_{1}+O\left(\varepsilon^{2}\right)
$$

in equations (2.3.3)-(2.3.4) and collecting terms of order 1 , one obtains the so-called $O(1)$ problem. Likewise, collecting terms of order $\varepsilon$ yields the $O(\varepsilon)$ problem. The $O(1)$ and $O(\varepsilon)$ problem will be treated separately in the following sections.

\subsubsection{The $O(1)$ problem}

The $O(1)$ problem is linear and reads

$$
\begin{aligned}
& (1+\beta) \frac{\partial \zeta_{0}}{\partial t}+\frac{\partial u_{0}}{\partial x}=0 \\
& \frac{\partial u_{0}}{\partial t}+\frac{\partial \zeta_{0}}{\partial x}+r u_{0}=0
\end{aligned}
$$

hence it admits nontransient solutions of the form

$$
\left(\zeta_{0}(x, t), u_{0}(x, t)\right)=\operatorname{Re}\left\{\left(\hat{\zeta}_{0}(x), \hat{u}_{0}(x)\right) e^{-i t}\right\}
$$

with $\hat{\zeta}_{0}(x)$ and $\hat{u}_{0}(x)$ complex amplitudes and $\operatorname{Re}\{\cdot\}$ denoting the real part of a complex number. Equation (2.3.10) describes the primary tidal wave in the system. By eliminating $u_{0}$ from Eqs. (2.3.8)-(2.3.9) and substituting $\zeta_{0}$ from equation (2.3.10), an ordinary differential equation (ODE) for $\hat{\zeta}_{0}$ is found:

$$
\frac{\partial^{2} \hat{\zeta}_{0}}{\partial x^{2}}+k^{2} \hat{\zeta}_{0}=0
$$

with complex wave number $k$ satisfying $k^{2}=(1+\beta)(1+i r)$. This has the general solution

$$
\hat{\zeta}_{0}(x)=A e^{i k x}+B e^{-i k x},
$$

where $A$ and $B$ are complex-valued integration constants. When equation (2.3.12) is substituted into equation (2.3.10), and choosing the root with positive real part (i.e, $\operatorname{Re}\{k\}>0$ ), it follows that the first term on the right hand side of equation (2.3.12) describes the spatial structure of a right-propagating wave, while the second term describes the structure of a left-propagating wave. Equations (2.3.8)-(2.3.9) allow for solutions that consist of waves propagating in opposite directions. Following the considerations in Section 2.2, conditions at both the left $(x=-L / 2)$ and right $(x=L / 2)$ boundary are imposed on the 
waves that enter the domain. At the left boundary, the incoming wave progressing to the right has a sea surface elevation amplitude $z_{l}$. At the right boundary a left-propagating tidal wave enters with a sea surface elevation amplitude of $z_{r}$. The phase difference between the incoming tidal waves at the boundary is $\theta$. Hence, the sea surface variations corresponding to right-propagating wave at $x=-L / 2$ and the left-propagating wave at $x=L / 2$ are, respectively,

$$
z_{l} \cos (\operatorname{Re}\{k\} x-t) \text { and } z_{r} \cos (\operatorname{Re}\{k\} x+t-\theta) .
$$

Thus, high water related to the incoming wave at the left boundary occurs at time $t=-\operatorname{Re}\{k\} L / 2$ and at the right boundary it occurs a time $\theta$ later. These boundary conditions are met by choosing

$$
A=z_{l} e^{-\operatorname{Im}\{k\} \frac{L}{2}} \quad \text { and } \quad B=z_{r} e^{-\operatorname{Im}\{k\} \frac{L}{2}+i \theta},
$$

with $\operatorname{Im}\{\cdot\}$ denoting the imaginary part of a complex number. Note that, when $\theta=0$ and $z_{l}=z_{r}$, the two incoming waves have equal phase and amplitude. In that symmetric case, the velocity $u_{0}$ vanishes in the middle of the channel (at $x=0$ ) and water motion in half the embayment (from $x=-L / 2$ to $x=0$ ) behaves as if in a semi-enclosed embayment with the left-propagating wave representing the wave reflected at the closed boundary. Once the sea surface elevation $\zeta_{0}$ is known, the along-channel current velocity $u_{0}$ follows from either equation (2.3.8) or Eq. (2.3.9) using Eq. (2.3.10),

$$
\hat{u}_{0}(x)=\frac{i k}{i-r}\left(A e^{i k x}-B e^{-i k x}\right) .
$$

As mentioned in Section 2.2.2, the friction parameter $r$ is determined from the Lorentz linearization condition (2.3.7). First, $u_{0}$ is calculated with equation (2.3.14)-(2.3.10), using an initial guess for $r$. Next, $r$ is updated to satisfy equation (2.3.7), with $u=u_{0}$ and the integrals are calculated numerically. A new value for $u_{0}$ is calculated using this updated value for $r$, and the process is iterated until the relative change in $r$ between successive iterations is less than $0.0001 \%$. This $r$-value is also used in the $O(\varepsilon)$ problem below.

\subsubsection{The $O(\varepsilon)$ problem}

The $O(\varepsilon)$ problem reads

$$
\begin{aligned}
(1+\beta) \frac{\partial \zeta_{1}}{\partial t}+\frac{\partial u_{1}}{\partial x} & =\underbrace{-\frac{\partial}{\partial x}\left(u_{0} \zeta_{0}\right)}_{\mathrm{I}} \underbrace{+\beta \alpha\left(1-\zeta_{0}\right) \frac{\partial \zeta_{0}}{\partial t}}_{\mathrm{II}}, \\
\frac{\partial u_{1}}{\partial t}+\frac{\partial \zeta_{1}}{\partial x}+r u_{1} & =\underbrace{-u_{0} \frac{\partial u_{0}}{\partial x}}_{\mathrm{III}} \underbrace{r u_{0} \zeta_{0}}_{\mathrm{IV}} \underbrace{+\beta u_{0} \frac{\partial \zeta_{0}}{\partial t} \mathcal{H}\left(-\frac{\partial \zeta_{0}}{\partial t}\right)}_{\mathrm{V}} .
\end{aligned}
$$




\begin{tabular}{ll}
\hline Term & Source \\
\hline I & divergence of excess mass \\
II & mass storage \\
III & advection of momentum \\
IV & depth dependent bottom friction \\
V & momentum sink \\
\hline
\end{tabular}

Table 2.1: Source of the forcing terms.

These equations have the same form as those of the $O(1)$ problem, but with the known $O(1)$ solutions forcing the unknowns $u_{1}$ and $\zeta_{1}$ through the inhomogeneous terms on the right hand side of equations (2.3.15)-(2.3.16). Every term in the inhomogeneity originates from nonlinear terms in Eqs. (2.3.4)-(2.3.3). In Table 2.1, the physical source of every nonlinear term is presented.

Since the inhomogeneities I, II, III and IV in the $O(\varepsilon)$ problem consist of products of the $O(1)$ solutions, which themselves are $\mathrm{M}_{2}$ signals, they are composed of $\mathrm{M}_{0}$ and $\mathrm{M}_{4}$ harmonics. Term $\mathrm{V}$ is different from terms $\mathrm{I}-\mathrm{IV}$ in the sense that it is not quadratic. Because of the presence of the discontinuous step function $\mathcal{H}$, that term consists of an infinite number of harmonics,

$$
\beta u_{0} \frac{\partial \zeta_{0}}{\partial t} \mathcal{H}\left(-\frac{\partial \zeta_{0}}{\partial t}\right)=\sum_{m=-\infty}^{\infty} p_{m} e^{-i m t}
$$

where the complex coefficients $p_{m}$ are given in Appendix 2.7.2. Note that $p_{m}$ depends in a nonlinear way on $\beta$ through $\zeta_{0}$ and $u_{0}$. The nontransient solution of the $O(\varepsilon)$ problem has the form

$$
\left(\zeta_{1}(x, t), u_{1}(x, t)\right)=\left(\left\langle\zeta_{1}\right\rangle(x),\left\langle u_{1}\right\rangle(x)\right)+\sum_{m=1}^{\infty} \operatorname{Re}\left\{\left(\hat{\zeta}_{1, m}(x), \hat{u}_{1, m}(x)\right) e^{-i m t}\right\} .
$$

In the remainder of this section, $\left\langle\zeta_{1}\right\rangle,\left\langle u_{1}\right\rangle, \hat{\zeta}_{1, m}$ and $\hat{u}_{1, m}$ are calculated.

The residual current velocity $\left\langle u_{1}\right\rangle$ is determined by averaging equation (2.3.15) over a tidal cycle using the condition that there is no net transport of water, $\langle u(1+\varepsilon \zeta)\rangle=0$. So, the residual current only compensates for Stokes transport created by the tidal wave. This yields

$$
\left\langle u_{1}\right\rangle=-\left\langle u_{0} \zeta_{0}\right\rangle=\underbrace{-\frac{1}{2}\left|\hat{u}_{0}\right|\left|\hat{\zeta}_{0}\right| \cos \left(\phi_{\hat{u}_{0}}-\phi_{\hat{\zeta}_{0}}\right)}_{\text {I }},
$$

where $\left|\hat{u}_{0}\right|$ and $\phi_{\hat{u}_{0}}$ are the amplitude and phase of the $O(1)$ current velocity $\hat{u}_{0}$. Likewise, $\left|\hat{\zeta}_{0}\right|$ and $\phi_{\hat{\zeta}_{0}}$ are the amplitude and phase of the $O(1)$ sea surface elevation $\hat{\zeta}_{0}$. 
The residual sea surface elevation $\left\langle\zeta_{1}\right\rangle$ results from the tidal average of Eq. (2.3.16) and imposing the condition that the average volume of water over one tidal period remains the same, $\int_{-L / 2}^{L / 2}\left\langle\zeta_{1}\right\rangle d x=0$,

$$
\left\langle\zeta_{1}\right\rangle=-\frac{1}{L} \int_{-L / 2}^{L / 2} \int_{0}^{x} E(s) d s d x+\int_{0}^{x} E(s) d s
$$

with

$$
E(x)=\underbrace{-\frac{1}{4} \frac{\partial\left|\hat{u}_{0}\right|^{2}}{\partial x}}_{\text {III }}+\left|\hat{u}_{0}\right|\left|\hat{\zeta}_{0}\right|(\underbrace{r \cos \left(\phi_{\hat{u}_{0}}-\phi_{\hat{\zeta}_{0}}\right)}_{\text {IV }}-\underbrace{\frac{\beta}{4} \sin \left(\phi_{\hat{u}_{0}}-\phi_{\hat{\zeta}_{0}}\right)}_{\mathrm{V}}) .
$$

In equation (2.3.20), the variable $s$ is used as a dummy variable for $x$. It follows from equation (2.3.21) that advection of momentum (III), depth dependent bottom friction (IV) and momentum sink (V) lead to residual sea surface elevation.

In order to obtain solutions for $\hat{\zeta}_{1, m}$, equation (2.3.18) is substituted into equations (2.3.15)-(2.3.16). Subsequently, $u_{1}$ is eliminated, which results in an ODE for every $\hat{\zeta}_{1, m}$,

$$
\frac{\partial^{2} \hat{\zeta}_{1, m}}{\partial x^{2}}+\left(k_{m}\right)^{2} \hat{\zeta}_{1, m}=f_{m}(x),
$$

with $\left(k_{m}\right)^{2}=(1+\beta) m(m+r i)$ and

$$
\begin{aligned}
f_{1} & =\underbrace{2 \beta \alpha \hat{\zeta}_{0}}_{\mathrm{II}} \underbrace{+2 \frac{\partial p_{1}}{\partial x}}_{\mathrm{V}}, \\
f_{2} & =\underbrace{-i \frac{\partial\left(\hat{u}_{0} \hat{\zeta}_{0}\right)}{\partial x}}_{\mathrm{I}} \underbrace{-\frac{1}{4} \frac{\partial^{2}}{\partial x_{0}^{2}}}_{\mathrm{III}} \underbrace{+r \frac{\partial\left(\hat{u}_{0} \hat{\zeta}_{0}\right)}{\partial x}}_{\mathrm{IV}} \underbrace{-2 \beta \alpha \hat{\zeta}_{0}^{2}}_{\mathrm{II}} \underbrace{+2 \frac{\partial p_{2}}{\partial x}}_{\mathrm{V}}, \\
f_{m \geq 3} & =\underbrace{2 \frac{\partial p_{m}}{\partial x}}_{\mathrm{V}} .
\end{aligned}
$$

It follows from equations (2.3.23)-(2.3.25) that advection of momentum (III), depth dependent bottom friction (IV) and divergence of excess mass (I) create an $\mathrm{M}_{4}$ harmonic in the sea surface elevation. The mass storage on the tidal flats (II) generates $\mathrm{M}_{2}$ and $\mathrm{M}_{4}$. The momentum sink (V) generates $\mathrm{M}_{2}, \mathrm{M}_{4}$, $\mathrm{M}_{6}, \mathrm{M}_{10}, \mathrm{M}_{14}, \mathrm{M}_{18}, \ldots$ The momentum sinks skips generation of $\mathrm{M}_{8}, \mathrm{M}_{12}$, $\mathrm{M}_{16}, \ldots$, because $p_{m}=0$ if $m>2$ and even. Solutions of the ODEs in Eq. (2.3.22) are obtained using variation of parameters,

$$
\begin{aligned}
\hat{\zeta}_{1, m}= & C_{m} e^{i k_{m} x}+D_{m} e^{-i k_{m} x} \\
& +\frac{e^{i k_{m} x}}{2 i k_{m}} \int_{-L / 2}^{x} e^{-i k_{m} s} f_{m}(s) d s+\frac{e^{-i k_{m} x}}{2 i k_{m}} \int_{x}^{L / 2} e^{i k_{m} s} f_{m}(s) d s,
\end{aligned}
$$


in which $C_{m}$ and $D_{m}$ are complex-valued integration constants. The boundary conditions for the $O(\varepsilon)$ problem $(2.3 .15)-(2.3 .16)$ are similar as those for the $O(1)$ problem. At $x=-L / 2$, it is imposed that the $\mathrm{M}_{2 m}$ tidal wave that enters the domain has sea surface amplitude $z_{l, m}$. Similarly, at the right boundary $(x=L / 2)$ a left-propagating $\mathrm{M}_{2 m}$ tidal wave enters with a given sea surface amplitude $z_{r, m}$ and a phase shift $\theta_{m}$ with respect to the incoming wave at the left boundary. These conditions are met when

$$
C_{m}=z_{l, m} e^{-\operatorname{Im}\left\{k_{m}\right\} \frac{L}{2}} \quad \text { and } D_{m}=z_{r, m} e^{-\operatorname{Im}\left\{k_{m}\right\} \frac{L}{2}+i \theta_{m}} .
$$

To study the overtides generated inside the domain by incoming $\mathrm{M}_{2}$ waves, $z_{l, m}$ and $z_{r, m}$ are chosen to be zero, except when the comparison is made with a complex numerical model in Section 2.5.3.

The complex amplitudes $\hat{u}_{1, m}$ of the $O(\varepsilon)$ current velocity $u_{1}$ are found by substituting Eq. (2.3.18) in Eq. (2.3.16). This yields

$$
\begin{aligned}
\hat{u}_{1,2} & =\frac{1}{2 i-r}(\underbrace{\frac{1}{4} \frac{\partial \hat{u}_{0}^{2}}{\partial x}}_{\mathrm{III}}+\frac{\partial \hat{\zeta}_{1,2}}{\partial x} \underbrace{-\frac{r}{2} \hat{u}_{0} \hat{\zeta}_{0}}_{\mathrm{IV}} \underbrace{-2 p_{2}}_{\mathrm{V}}), \\
\hat{u}_{1, m \neq 2} & =\frac{1}{i m-r}(\frac{\partial \hat{\zeta}_{1, m}}{\partial x}-\underbrace{2 p_{m}}_{\mathrm{V}}) .
\end{aligned}
$$

It follows from these expressions that advection of momentum (III), depth dependent bottom friction (IV) create $\mathrm{M}_{4}$ velocities and the momentum sink (V) generates $\mathrm{M}_{2}, \mathrm{M}_{4}, \mathrm{M}_{6}, \mathrm{M}_{10}, \ldots$ velocities.

\subsection{Results}

In order to address the two objectives set out in Section 2.1, parameter values will be chosen that are representative of a prototype double inlet system, viz. the Marsdiep-Vlie system in the Western Wadden Sea. The embayment is roughly $60 \mathrm{~km}$ long, $15 \mathrm{~km}$ wide and the channel has an averaged depth of $10 \mathrm{~m}$. The primary tide is the semi-diurnal lunar tide $\mathrm{M}_{2}$ and its first overtide $\mathrm{M}_{4}$ is clearly present (Buijsman and Ridderinkhof, 2007). The total tidal range is around $1.4 \mathrm{~m}$ at the Marsdiep and $1.8 \mathrm{~m}$ at the Vlie and the moment of high water at the Marsdiep differs by approximately 90 minutes from that at the Vlie (Royal Netherlands Navy, Hydrographic Service, 2016). The default parameter values used are listed in Table 2.2.

\subsubsection{Impact of the momentum sink on tides in the de- fault setting}

In Figure 2.2, results are shown of the primary tide $\mathrm{M}_{2}$ and its first overtide $\mathrm{M}_{4}$ of the sea surface elevation and current velocity in the regime where all forcing 


\begin{tabular}{lll}
\hline Parameter & dimensionless & dimensional \\
\hline$L$ & $\frac{2 \pi}{7.8}$ & $57 \mathrm{~km}$ \\
$c_{d}$ & 0.0025 & \\
$z_{l}, z_{r}$ & 0.7 & $0.7 \mathrm{~m}$ \\
$d_{f}$ & 1 & $1 \mathrm{~m}$ \\
$z_{l, m}, z_{r, m}$ & 0 & $0 \mathrm{~m}$ \\
$\theta$ & $\frac{\pi}{4}$ & $93 \mathrm{~min}$ \\
$\theta_{m}$ & 0 & $0 \mathrm{~min}$ \\
$h_{c}$ & & $10 \mathrm{~m}$ \\
$g$ & & $10 \mathrm{~m} \mathrm{~s} \mathrm{~s}^{-1}$ \\
$\sigma$ & 0.1 & $0.00014 \mathrm{~s}^{-1}$ \\
$\varepsilon=\frac{\mathcal{Z}}{h_{c}}$ & 2 & \\
$\beta=\frac{b_{\max }-b_{c}}{b_{c}}$ & 2 & \\
$\alpha=\frac{1}{2 q \varepsilon}$ & 1 & \\
\hline
\end{tabular}

Table 2.2: Default setting of parameters, representative of the Marsdiep-Vlie embayment. Sources for these values are Dronkers (2005) and Royal Netherlands Navy, Hydrographic Service (2016).

terms in equations (2.3.15)-(2.3.16) are taken into account and for the case where the momentum sink is turned off (term $\mathrm{V}=0$ ). Panels (a) and (c) of this figure reveal that including the momentum sink lowers the $\mathrm{M}_{2}$ amplitude and increases the $\mathrm{M}_{4}$ amplitude of both the sea surface elevation and the current velocity of the order of centimeters, respectively centimeters per second. For the $\mathrm{M}_{2}$ amplitudes, that change is approximately $2 \%$, for the $\mathrm{M}_{4}$ amplitudes, approximately $25 \%$. The increase in absolute value of the residual sea surface elevation due to the momentum sink is of the order of $0.5 \mathrm{~cm}$, corresponding to a relative change of $30 \%$. Note that in Figure $2.2 \mathrm{a}$ and $2.2 \mathrm{c}$ the $\mathrm{M}_{2}$ amplitude of both the sea surface elevation and the current velocity at the left boundary differs from that at the right boundary, even though the amplitudes of the incoming tidal waves at the boundaries are equal.

Figures $2.2 \mathrm{~b}$ and $2.2 \mathrm{~d}$ reveal that the $\mathrm{M}_{2}$ phase of both the sea surface elevation and the current velocity is hardly influenced. However, the phase of the $\mathrm{M}_{4}$ component of the sea surface elevation is lowered by approximately 15 degrees. The phase of the $\mathrm{M}_{4}$ harmonic of the velocity is lowered by approximately 10 degrees. Figure 2.2b illustrates that the $\mathrm{M}_{2}$ sea surface elevation has the character of a propagating wave near the boundaries, but it turns into a standing wave in the middle of the channel as the phase becomes constant there. The $\mathrm{M}_{4}$ component of the sea surface elevation has the character of a standing wave with a jump in phase around $x^{*}=-6 \mathrm{~km}$. Both the $\mathrm{M}_{2}$ and $\mathrm{M}_{4}$ components of the current velocity have the character of standing waves with jumps in phase around $x^{*}=15 \mathrm{~km}$. 


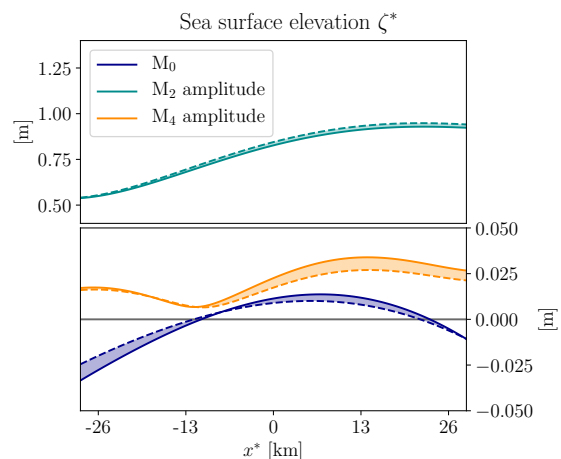

(a)

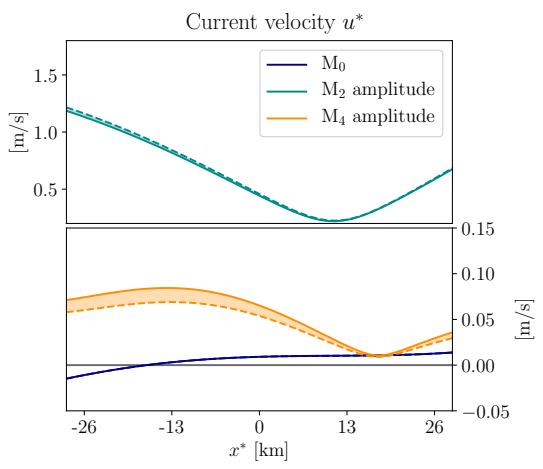

(c)

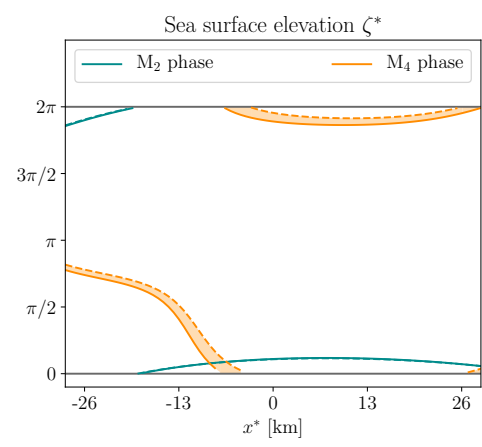

(b)

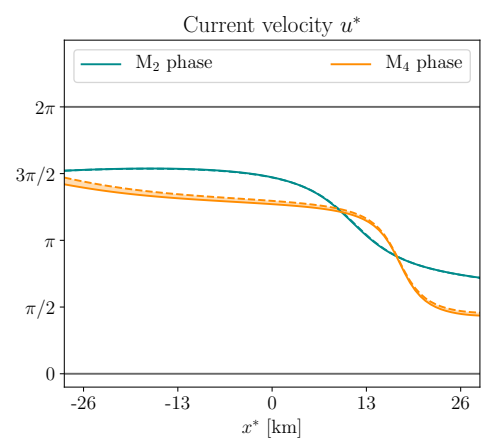

(d)

Figure 2.2: $(\mathrm{a}, \mathrm{b})$ Amplitude and phase of the sea surface elevation $\zeta^{*}$ versus distance $x^{*}$, for different harmonics $\mathrm{M}_{0}, \mathrm{M}_{2}$ and $\mathrm{M}_{4}$ and for the default parameter setting (Table 2.2). The solid line represents the solution accounting for all the nonlinear terms $(\mathrm{I}-\mathrm{V})$, including the momentum sink (term V). The dashed line represents the solution with the momentum sink neglected. The shaded area marks the difference between them. (c, d) As (a) and (b), but for the current velocity $u^{*}$. 


\subsubsection{Impact of the momentum sink on sediment trans- port}

Changes in tidal harmonics as described above may lead to a difference in sediment transport. The net sediment transport due to the primary tide and its first overtide is calculated as in de Swart and Zimmerman (2009) ${ }^{1}$ (after Groen, 1967),

$$
\begin{aligned}
\left\langle S^{*}\right\rangle= & \frac{\nu^{*}}{4 \gamma^{*}} U_{M_{2}}^{* 2} U_{M_{4}}^{*} \cos (\tau)\left(\frac{2}{1+a^{2}}+\frac{1}{1+4 a^{2}}\right) \\
& -a \frac{\nu^{*}}{2 \gamma^{*}} U_{M_{2}}^{* 2} U_{M_{4}}^{*} \sin (\tau)\left(\frac{1}{1+a^{2}}-\frac{1}{1+4 a^{2}}\right),
\end{aligned}
$$

with $\nu^{*}\left(\mathrm{~s} \mathrm{~m}^{-1}\right)$ an erosion parameter and $\gamma^{*}\left(\mathrm{~s}^{-1}\right)$ a settling parameter $\left(1 / \gamma^{*}\right.$ is the timescale in which sediment settles to the bed), $a=\sigma^{*} / \gamma^{*}, \tau=$ $\arg \left(\varepsilon \hat{u}_{1,2}\right)-2 \arg \left(\hat{u}_{0}+\varepsilon \hat{u}_{1,1}\right)$ the relative phase of the $\mathrm{M}_{2}$ and $\mathrm{M}_{4}$ harmonic, and

$U_{M_{2}}^{*}=\mathcal{U}\left|\hat{u}_{0}+\varepsilon \hat{u}_{1,1}\right|$ and $U_{M_{4}}^{*}=\mathcal{U}\left|\varepsilon \hat{u}_{1,2}\right|$ the $\mathrm{M}_{2}$ and $\mathrm{M}_{4}$ amplitude of the current velocity. Coarse sediment is characterized by a small $a \approx 0.01$, while $a \approx 5$ is representative for fine sediment. Figure 2.3 shows the tidally averaged volumetric sediment transport per unit mass and width $\left\langle S^{*}\right\rangle$, divided by $\nu^{*} / 4 \gamma^{*}$, both for when the momentum sink is included and when it is not. The purple lines shows the situation with coarse sediment and the red ones the situation with fine sediment. The arrows indicate the direction of the sediment transport. The figure reveals that including the momentum sink increases the absolute value of the transport of coarse sediment by approximately $35 \%$ and has little impact on the transport of fine sediment. On the left (right) side of the channel, the flood current is to the right (left). Hence, the momentum sink increases transport of coarse sediment in the flood direction, that is, it leads to an import of coarse sediment. Furthermore, Figure 2.3 shows that fine sediment is transported through the embayment, from the right boundary to the left boundary. Coarse sediment accumulates around $x^{*}=5 \mathrm{~km}$ and suggests the formation of a tidal watershed at this location.

\subsubsection{Sensitivity study}

In this section it is investigated how sensitive the results of the preceding section are to changes in model parameters. The difference in amplitude due to the momentum sink is henceforth denoted by $\Delta$ Ampl.

Figure 2.4 shows $\Delta \mathrm{Ampl}$ in sea surface elevation for various harmonics as a function of $x^{*}$ and for different values of flat-to-channel area ratio $\beta$, slope

\footnotetext{
${ }^{1}$ The second plus sign in $\left(1 /\left(1+a^{2}\right)+1 /\left(1+4 a^{2}\right)\right)$ is a typo in de Swart and Zimmerman (2009), copied in Hepkema et al. (2018). Figure 2.3 is made with the corrected equation (2.4.1); the difference is minimal.
} 


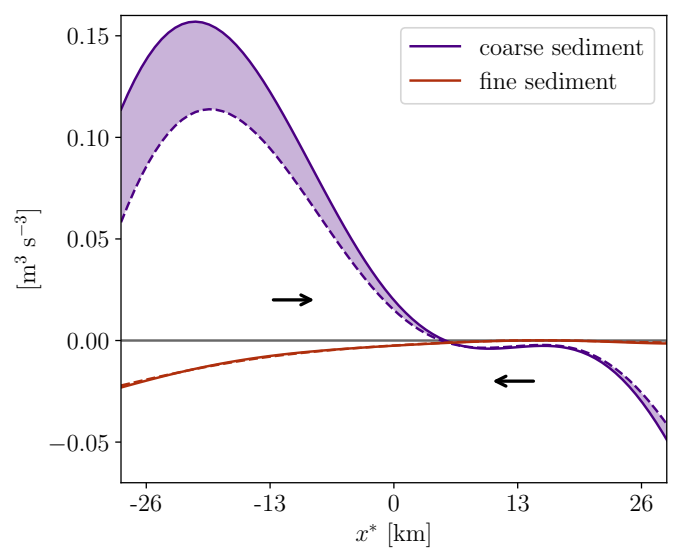

Figure 2.3: Tidally averaged sediment transport $\left\langle S^{*}\right\rangle$, divided by $(\nu / 4 \gamma)$, versus distance $x^{*}$. The purple lines shows the transport of coarse sediment, $a=0.01$ and red the one of fine sediment, $a=5$. The solid line represents the solution with the momentum sink taken into account and the dashed line the solution where it is not. The arrows illustrate the direction of the sediment transport and indicate the formation of a shoal at $x^{*}=5 \mathrm{~km}$. Values of the remaining parameters are those in Table 2.2.

parameter $\alpha$ and drag coefficient $c_{d}$. Red colours denote an increase and blue colours a decrease in amplitude. Figure 2.5 is similar to Figure 2.4, but for the current velocity instead of the sea surface elevation. The difference in phase due to the momentum sink is not shown since it remains close to zero for the $\mathrm{M}_{2}$ harmonic and close to 10-15 degrees for the $\mathrm{M}_{4}$ harmonic when the parameters $\beta, \alpha$ and $c_{d}$ are varied. The $\mathrm{M}_{0}$ component of the current velocity is also left out since equation (2.3.19) already shows that $\Delta \mathrm{Ampl}=0$.

What stands out in Figs. $2.4-2.5$ is that $\Delta \mathrm{Ampl}$ in both the sea surface elevation and current velocity is most sensitive to changes in flat-to-channel area ratio $\beta$, and less to changes in slope parameter $\alpha$ and drag coefficient $c_{d}$. Figure 2.4 reveals that the momentum sink lowers the $\mathrm{M}_{2}$ amplitude and increases the $\mathrm{M}_{4}$ one for almost all $\beta$-values considered. Furthermore, it illustrates that $\Delta \mathrm{Ampl}$ depends nonlinearly on $\beta$. In particular, the sensitivity of $\Delta \mathrm{Ampl}$ to $\beta$ decreases, as $\beta$ increases. Figure 2.5 shows that these conclusions also hold for the current velocity.

Furthermore, Figure 2.4 reveals that, although $\Delta \mathrm{Ampl}$ in $\mathrm{M}_{0}$ and $\mathrm{M}_{2}$ are rather insensitive to changes in $\alpha$, the response of the $\mathrm{M}_{4}$ harmonic of the sea surface elevation on changing $\alpha$-values is more interesting. On the right side of the embayment, for small $\alpha$ the momentum sink increases (red colours) the $\mathrm{M}_{4}$ sea surface elevation amplitude while for larger values of $\alpha$ the $\mathrm{M}_{4}$ amplitude 
is decreased (blue colours). From the bottom panels of Figs. 2.4-2.5 it follows that, for smaller values of the drag coefficient, the momentum sink leads to a larger difference in sea surface elevation and current velocity amplitude.

The sensitivity of $\Delta \mathrm{Ampl}$ in sea surface elevation and current velocity to the embayment length $L^{*}$ and the phase difference $\theta$ between the incoming tidal waves is presented in Figs. 2.6 and 2.7. For increasing $\theta$-values, the spatial pattern of $\Delta$ Ampl shifts towards the right, and the $\mathrm{M}_{2}$ and $\mathrm{M}_{4}$ sea surface elevation amplitude and $\mathrm{M}_{4}$ current velocity amplitude decreases. Particularly noticeable about the sensitivity of $\Delta$ Ampl on $L^{*}$ is that the $\mathrm{M}_{2}$ sea surface elevation amplitude has a local maximum at $L^{*} \approx 45 \mathrm{~km}$. 


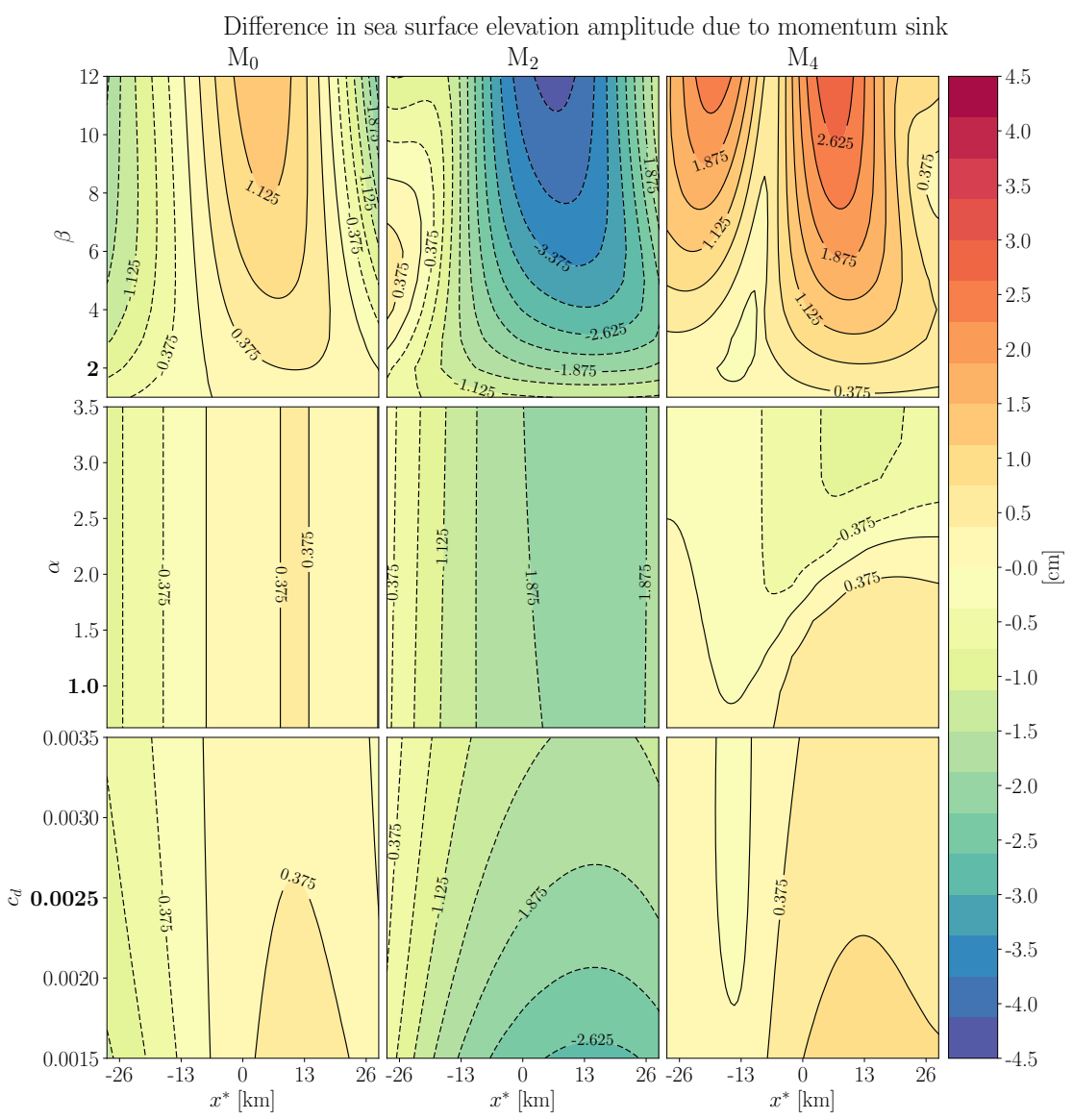

Figure 2.4: Contours of the momentum sink-induced amplitude difference $\Delta$ Ampl in sea surface elevation versus flat-to-channel area ratio $\beta$ (top row), slope parameter $\alpha$ (middle row), drag coefficient $c_{d}$ (bottom row) and along-channel variable $x^{*}$ for the $\mathrm{M}_{0}$ (left column), $\mathrm{M}_{2}$ (middle column) and $\mathrm{M}_{4}$ (right column) harmonics. The bold values on the vertical axis represent the default setting, $\beta=2, \alpha=1$ and $c_{d}=0.0025$. The remaining parameters have the same value as in Table 2.2. 
Difference in current velocity amplitude due to momentum sink

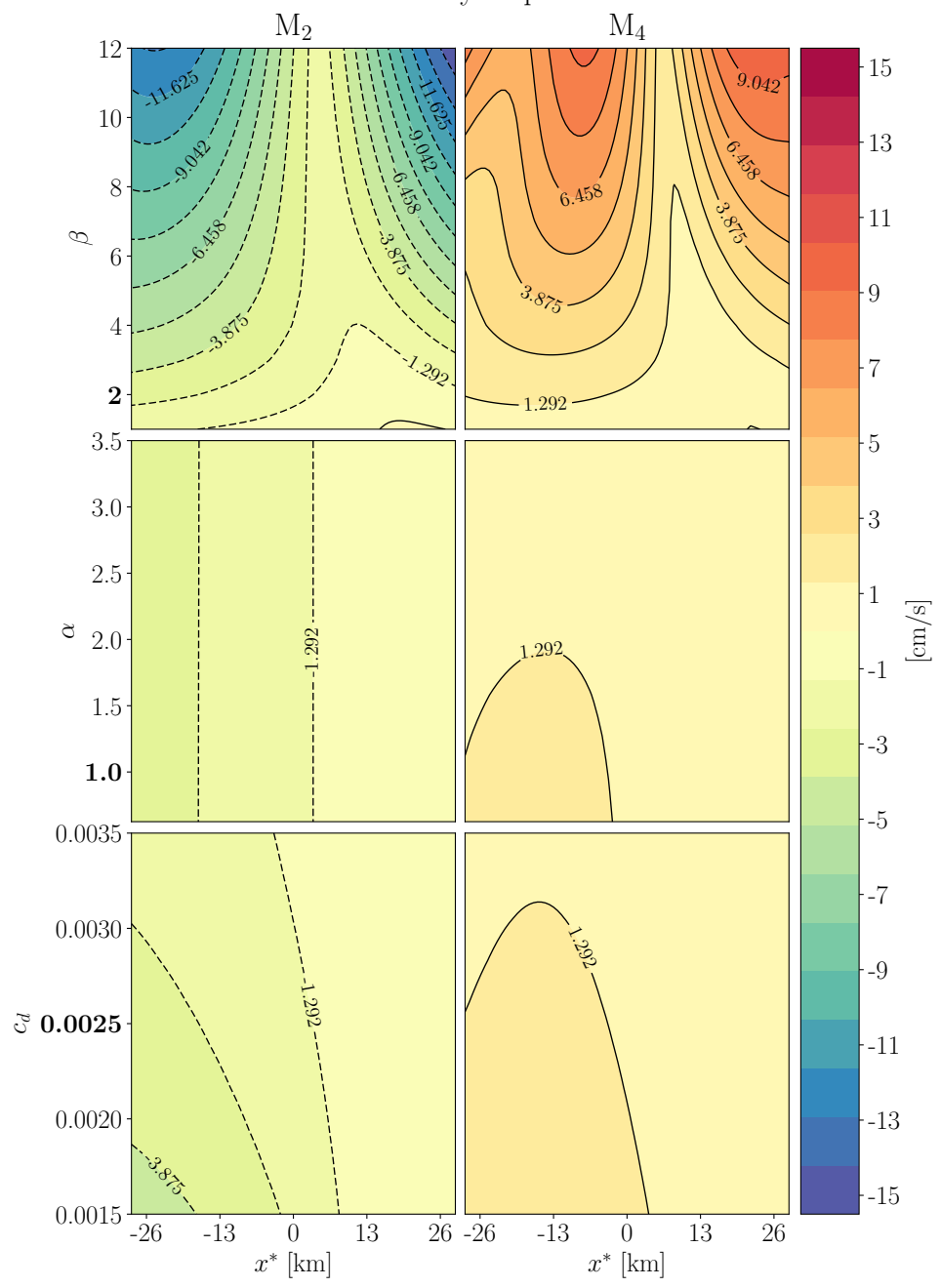

Figure 2.5: Contours of the momentum sink-induced amplitude difference $\Delta \mathrm{Ampl}$ in current velocity versus flat-to-channel area ratio $\beta$ (top row), slope parameter $\alpha$ (middle row), drag coefficient $c_{d}$ (bottom row) and along-channel variable $x^{*}$ for the $\mathrm{M}_{2}$ (left column) and $\mathrm{M}_{4}$ (right column) harmonics. The bold values on the vertical axis represent the default setting, $\beta=2, \alpha=1$ and $c_{d}=0.0025$. The remaining parameters have the same value as in Table 2.2 . 


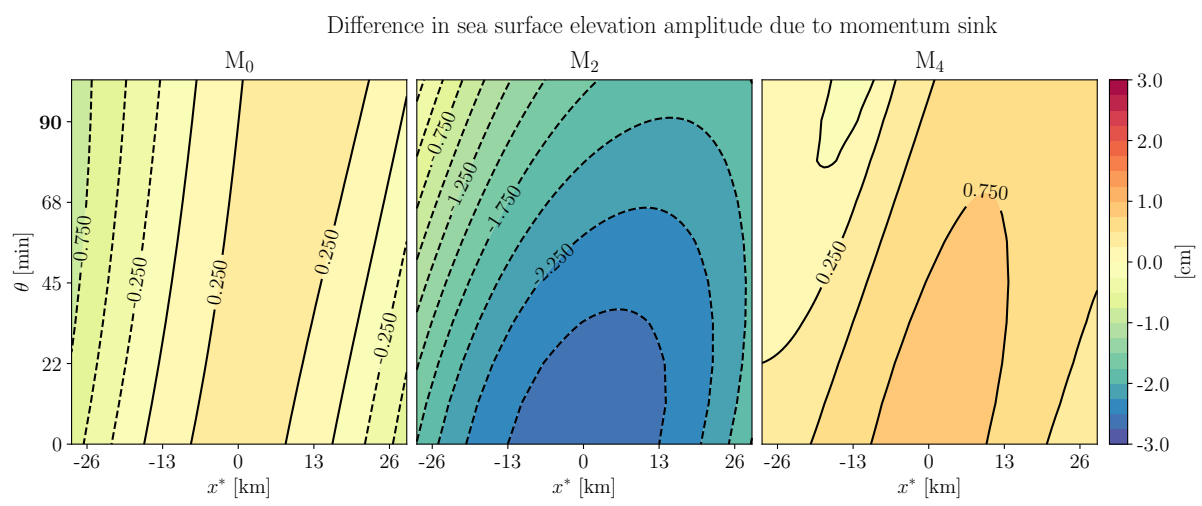

(a)

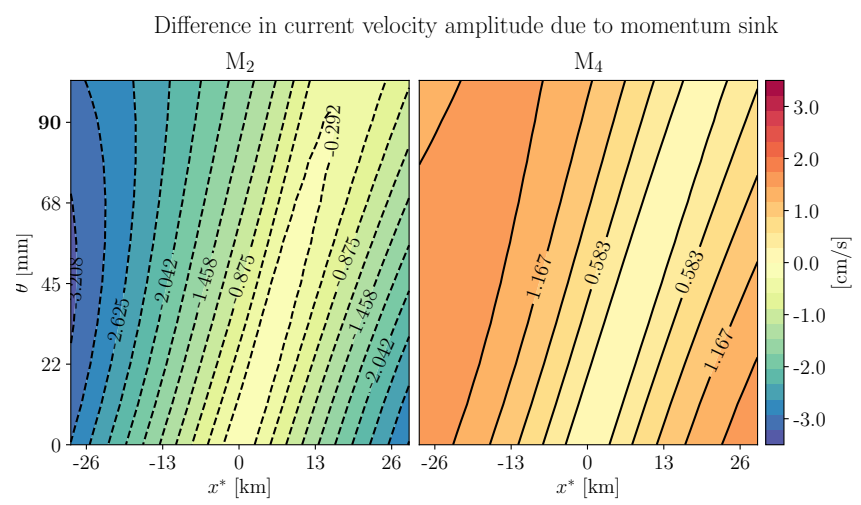

(b)

Figure 2.6: (a) Contours of the momentum sink-induced amplitude difference $\Delta$ Ampl, of the sea surface elevation versus the phase differences between the incoming tidal waves $\theta$ and along-channel variable $x^{*}$ for the $\mathrm{M}_{0}$ (left panel), $\mathrm{M}_{2}$ (middle panel) and $\mathrm{M}_{4}$ (right panel) harmonic. The bold value on the vertical axis represent the default setting $\theta=\pi / 4$. The remaining parameters have the same value as in Table 2.2. (b) As (a), but for the current velocity $u^{*}$. 

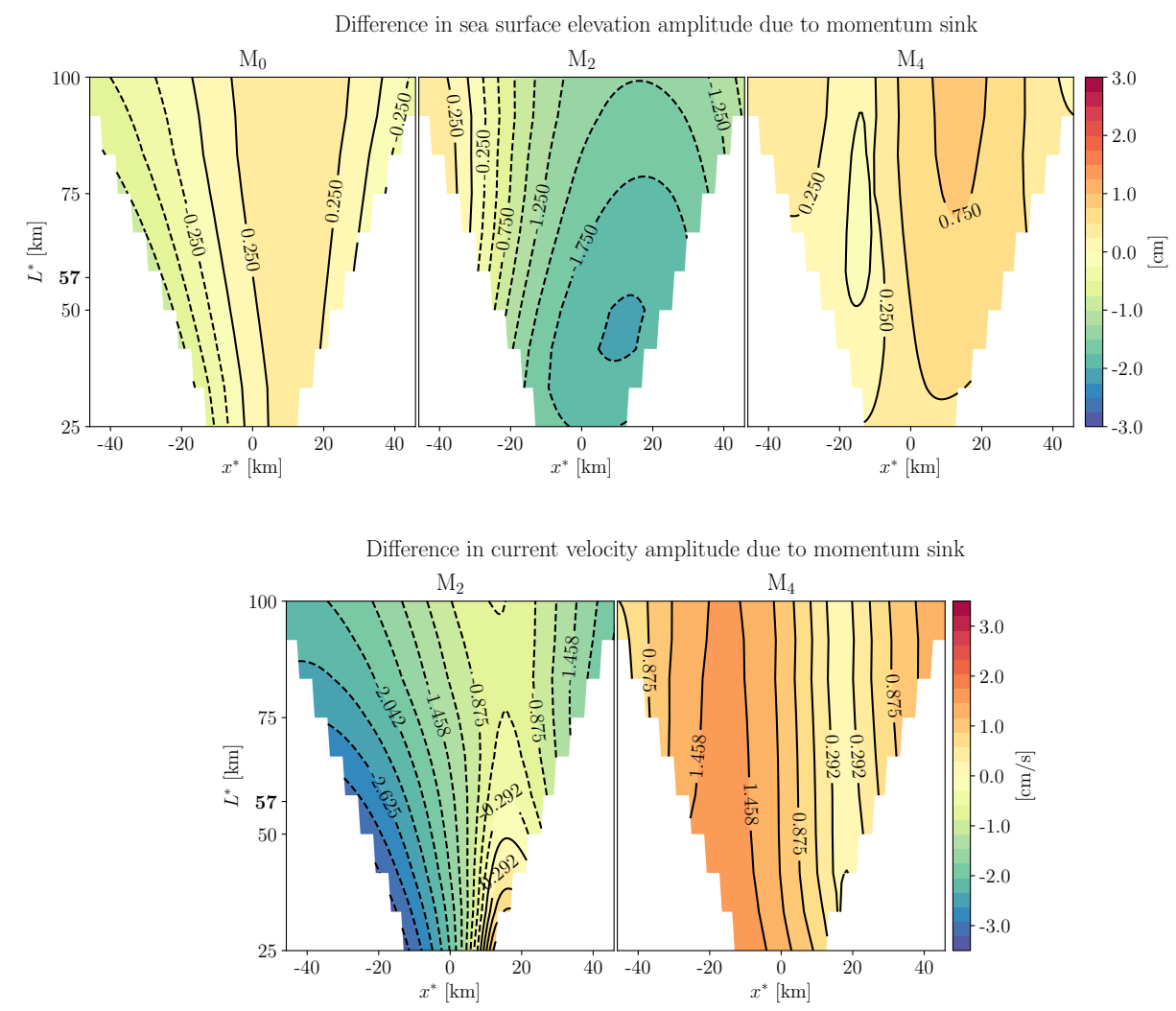

Figure 2.7: As Figure 2.6, but for different embayment lengths $L^{*}$. The bold value on the vertical axis represent the default setting $L^{*}=57 \mathrm{~km}$. 


\subsection{Discussion}

\subsubsection{Physical interpretation of the results}

In this section, some results obtained in the preceding section are interpreted by analyzing the expressions from Section 2.3. To that end, it is helpful to rewrite term $\mathrm{V}$ in the $O(\varepsilon)$ problem (2.3.16) using the continuity equation (2.3.8). That term becomes:

$$
\beta u_{0} \frac{\partial \zeta_{0}}{\partial t} \mathcal{H}\left(-\frac{\partial \zeta_{0}}{\partial t}\right)=\frac{-\beta}{1+\beta} u_{0} \frac{\partial u_{0}}{\partial x} \mathcal{H}\left(-\frac{\partial \zeta_{0}}{\partial t}\right) .
$$

This shows that the momentum sink is similar to the advection term when the tide falls and, in particular, that the momentum sink term is always smaller than the advection term, by virtue of $\beta /(1+\beta)=\left(b_{\max }^{*}-b_{c}^{*}\right) / b_{\max }^{*}<1$.

The impact of the momentum sink on the residual sea surface elevation becomes apparent by this similarity. In fact, since $\left\langle\mathcal{H}\left(-\partial \zeta_{0} / \partial t\right)\right\rangle=1 / 2$, integration over one tidal period of the $O(\varepsilon)$ momentum equation (2.3.16) using equation (2.5.1) results in

$$
\frac{\partial\left\langle\zeta_{1}\right\rangle}{\partial x}=-\frac{1}{4} \frac{\partial\left|\hat{u}_{0}\right|^{2}}{\partial x}(\underbrace{1}_{\text {III }}+\underbrace{\frac{\beta}{2(1+\beta)}}_{\text {V }})+\underbrace{\left|\hat{u}_{0}\right|\left|\hat{\zeta}_{0}\right| r \cos \left(\phi_{\hat{u}_{0}}-\phi_{\hat{\zeta}_{0}}\right)}_{\text {IV }} .
$$

It follows from this expression that the momentum sink increases the absolute value of the gradient of residual sea surface elevation. This clarifies the results in Figure 2.2a.

In Section 2.4, it was found that the momentum sink reduces the amplitude of the $\mathrm{M}_{2}$ harmonic for most parameter values. To understand why, note that term $M^{*}$ in equation (2.2.5) (which arises as term $\mathrm{V}$ in the $O(\varepsilon)$ momentum equation) represents a force per mass unit, as it appears on the right hand side in the dimensional momentum equation (2.2.4). During the falling tide, this force acts against the direction of the current (as shown in equation (2.5.1), term $\mathrm{V}$ and $u_{0}$ have opposite signs when $\partial \zeta_{0} / \partial t<0$ ), whilst during the rising of the tide it is inactive (term $\mathrm{V}$ is zero). In other words, when the water level drops, stagnant water enters the channel from the flats, thereby slowing the channel current. During the rising of the tide, water flows from the channel onto the flats, which leaves the channel current unaffected. All in all, this implies that term $\mathrm{V}$ reduces the current of the principal tidal component. The continuity equation (2.3.8) implies, in turn, that the same holds for the sea surface elevation.

In a system resembling the Marsdiep-Vlie system, the momentum sink increases the $\mathrm{M}_{4}$ amplitudes (Figure 2.2). This can also be explained using equation (2.5.1). This equation expresses that the momentum sink term is the product of a constant, the advection term and the step function. The advection 
term consists of an $\mathrm{M}_{0}$ and $\mathrm{M}_{4}$ harmonic and the step function consists of a $\mathrm{M}_{0}$ and odd harmonics. The $\mathrm{M}_{4}$ component of the momentum sink is therefore the product of the $\mathrm{M}_{4}$ harmonic of the advection term and the $\mathrm{M}_{0}$ harmonic of the step function. That is, the momentum sink generates an $\mathrm{M}_{4}$ harmonic with the same phase as the $\mathrm{M}_{4}$ generated by advection (see Appendix 2.7.2 for details). Ridderinkhof et al. (2014) showed that advection leads to stronger and shorter flood currents (flood dominance). The momentum sink therefore also favours flood dominance. Since Figure 2.3 shows that the transport of coarse sediment is in the flood direction, it implies that the current is flood dominant. Thus, the $\mathrm{M}_{4}$ generated by the momentum sink increases the total $\mathrm{M}_{4}$ current velocity amplitude.

Figure 2.4 showed that, when the slopes of the flats are small (large $\alpha$ ), the $\mathrm{M}_{4}$ amplitude of the sea surface elevation is decreased by the momentum sink while it is increased for the default value of $\alpha$. The reason for this is similar to the above. When $\alpha$ is large, the mass storage is the dominant mechanism producing the $\mathrm{M}_{4}$ overtide in the sea surface elevation. Since this term generates $\mathrm{M}_{4}$ that is out of phase with the $\mathrm{M}_{4}$ generated by the momentum sink, including the momentum sink reduces the total $\mathrm{M}_{4}$ amplitude of the sea surface elevation.

Figure 2.2 revealed that the amplitudes of the sea surface elevation (and the current velocity) are different at the two boundaries. In these experiments, the amplitudes of the incoming tidal waves are equal, so even then, the resulting amplitudes at the boundaries differ. This is already included in the linear dynamics. The $O(1)$ solution (2.3.12)-(2.3.14) of the linearized problem yields, if $z_{l}=z_{r}$, that

$$
\left|\hat{\zeta}_{0}(-L / 2)\right|^{2}-\left|\hat{\zeta}_{0}(L / 2)\right|^{2}=4 z_{l}^{2} e^{-\operatorname{Im}\{k\} L} \sin (\theta) \sin (\operatorname{Re}\{k\} L) .
$$

Hence, for equal amplitudes of incoming waves, the amplitude at the boundaries differ when $\theta$ and $\operatorname{Re}\{k(r, \beta)\} L$ are not a multiple of $\pi$, with the difference depending on the phase difference $\theta$, the friction parameter $r$, the length of the channel $L$ and the flat-to-channel area ratio $\beta$. Furthermore, it follows from equation (2.5.3) that, in a longer embayment, the difference in amplitude due to the phase difference of the incoming tidal waves, becomes smaller. Physically, this makes sense, since in a longer embayment, a wave needs more time to propagate from one side to the other. All this time it is subject to friction. Hence, for large $L, \hat{\zeta}_{0}( \pm L / 2)$ is mainly determined by the incoming wave. Figure 2.8 qualifies the difference $\left|\hat{\zeta}_{0}(-L / 2)\right|-\left|\hat{\zeta}_{0}(L / 2)\right|$ between the $O(1)$ sea surface elevation amplitude at the left and right boundary for different flatto-channel area ratios $\beta$ and phase differences $\theta$ between the incoming tidal waves. The difference varies between +10 and $-50 \mathrm{~cm}$ and is largest for small $\beta$ - and large $\theta$-values (blue area). 


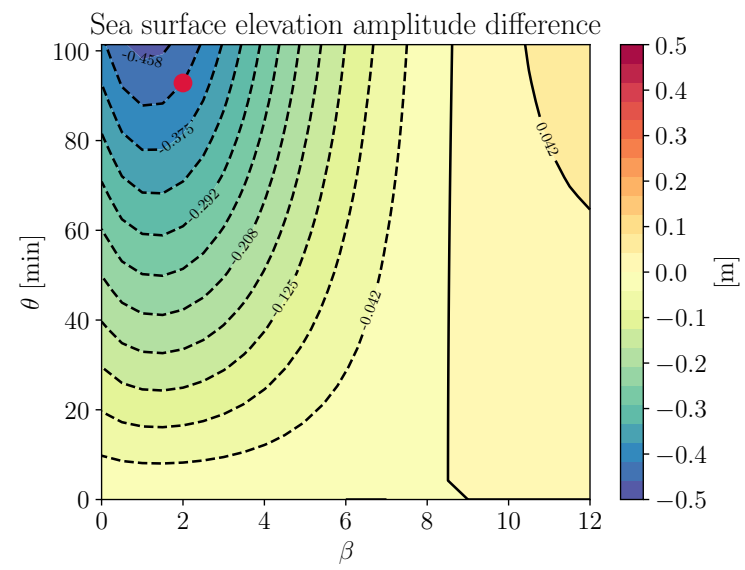

Figure 2.8: The difference between the $O(1)$ sea surface elevation amplitude at the left $(x=-L / 2)$ and right $(x=L / 2)$ boundary versus flat-to-channel area ratio $\beta$ and phase difference $\theta$ between the tidal waves entering the embayment at the left and right boundary. The remaining parameters have the same value as in Table 2.2 . In particular, the amplitudes $z_{l}$ and $z_{r}$ of the incoming tidal waves are equal. The red dot denotes the default value of $\beta$ and $\theta$.

\subsubsection{Model limitations}

In this study, the bottom stress is linearized to obtain analytically tractable (linear) $O(1)$ and $O(\varepsilon)$ problems. When the $\mathrm{M}_{2}$ tide is dominant, nonlinear bottom stress is known to especially generate odd overtides $\mathrm{M}_{6}, \mathrm{M}_{10}, \ldots$ (Parker, 1984). It is possible to include an approximation of the $\mathrm{M}_{6}$ generated by the nonlinear bottom stress. However, as stated in Friedrichs and Aubrey (1994), one-dimensional numerical models, even with nonlinear bottom stress, simulate observed $\mathrm{M}_{6}$ variations rather poorly. The authors remark that this might be due to the invalid assumption of a constant drag coefficient $c_{d}$. Therefore, as in Friedrichs and Aubrey (1994), the focus of this study is on the primary tide and its first overtide.

In natural tidal embayments, along-channel velocity $u$ is strongly reduced on the flats, but does not vanish completely. The choice of vanishing $u$ on the flats is made to consider the extreme case. As is shown in Section 2.4 there are (physically relevant) parameter regimes in which the momentum sink generates noticeably overtides. It would be interesting to relax this assumption in a future study.

The trapezoidal cross section is chosen to approximate concave-up tidal flats which typically occur in nature (Friedrichs, 2011). The approximation is such that the partly linear flat is tangential to the convex bed at $y^{*}= \pm b_{\max }^{*} / 2$ and $y^{*}= \pm b_{c}^{*} / 2$ (see Figure 2.9). In this study, steep slopes and wide flats 


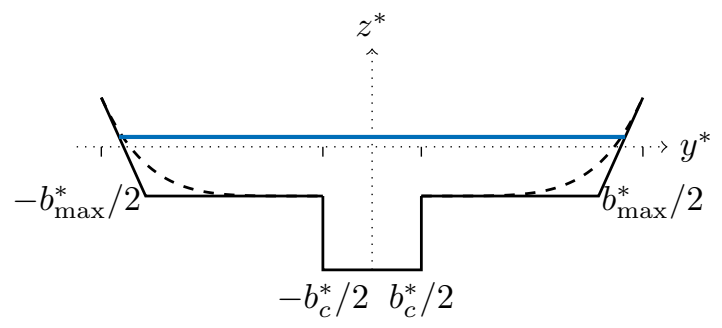

Figure 2.9: The trapezoidal cross section considered in the idealized model (solid line), which is an approximation of the concave-up flats (dashed line).

are chosen to obtain a system in which all nonlinear terms, and in particular both the momentum sink and the mass storage, are $O(\varepsilon)$, i.e., $\beta=O(1)$ and $q=O(1 / \varepsilon)$. Other choices are possible. For example, Speer and Aubrey (1985) considered moderate slopes and narrow flats, i.e., $\beta=O(\varepsilon)$ and $q=O(1)$. In that case, the momentum sink term is $O\left(\varepsilon^{2}\right)$ and the mass storage is $O(\varepsilon)$.

Furthermore, for simplicity, an embayment with a constant depth and width is chosen. Also, effects of wind, waves, density differences and radiation damping (as in Roos and Schuttelaars, 2015) are ignored. These are possible extensions of the model.

\subsubsection{Comparison with results of a complex numerical model}

In this section, the results of Sect. 2.4 are compared with hydrodynamics simulations of the Dutch Wadden Sea performed using the General Estuarine Transport Model (GETM). GETM is a three-dimensional finite difference model solving the primitive equations and includes a drying and flooding algorithm of the tidal flats. The resolution was $200 \mathrm{~m}$ horizontally, there were 30 vertical layers and a realistic bathymetry and forcing (see Duran-Matute et al. (2014) for further details and an extensive comparison with several tidal gauges).

A transect across the Western Wadden Sea is chosen (see Figure 2.10a) across which the tidal channels connect the Marsdiep and Vlie inlet (similar as in Ridderinkhof, 1988). In Figure 2.10b the $\mathrm{M}_{2}$ and $\mathrm{M}_{4}$ amplitude of the sea surface elevation in April 2009 (a calm month in terms of wind) along this transect are depicted together with the ones from the idealized model (as in Figure 2.2). The magnitude and spatial distribution of the $\mathrm{M}_{2}$ harmonic of the sea surface elevation as calculated by the idealized model and that of GETM roughly agree. The $\mathrm{M}_{4}$ amplitude calculated by GETM is approximately four times larger as that calculated by the idealized model. However, when the momentum sink is accounted for, the error becomes smaller (reduc- 


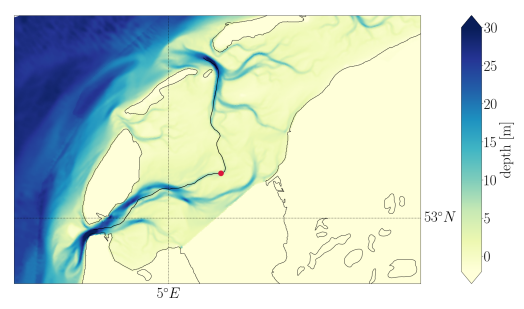

(a)

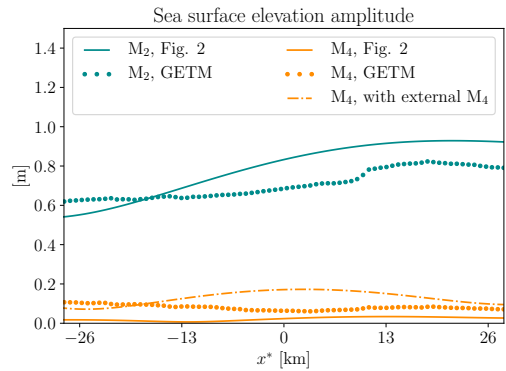

(b)

Figure 2.10: (a) Transect across the Marsdiep-Vlie inlet systems located in the Western Wadden Sea. Colours indicate the bathymetry used in GETM. (b) The $\mathrm{M}_{2}$ and $\mathrm{M}_{4}$ amplitudes of the sea surface elevation calculated by GETM on this transect (dotted lines). The solid lines are the $\mathrm{M}_{2}$ and $\mathrm{M}_{4}$ as in Figure 2.2 (including the momentum sink). The dashed-dotted line represents the $\mathrm{M}_{4}$ harmonic calculated by the idealized model when an external $\mathrm{M}_{4}$ tidal wave is included in the boundary conditions. The point $x^{*}=0$ is located by the red dot in panel (a).

tion of approximately 7\%). A possible explanation for this difference is that, in the idealized model, there is no $\mathrm{M}_{4}$ harmonic in the tidal wave entering the domain. In GETM, the $\mathrm{M}_{4}$ amplitude of the sea surface elevation $14 \mathrm{~km}$ northwest of Texel is approximately $0.082 \mathrm{~m}$. The dashed-dotted line in Figure $2.10 \mathrm{~b}$ represents the $\mathrm{M}_{4}$ harmonic of the sea surface elevation when such an $\mathrm{M}_{4}$ tidal wave entering at the boundaries is imposed. That is, the sea surface elevation of the right-propagating wave at $x=-L / 2$ and the left-propagating wave at $x=L / 2$ are chosen to be, respectively,

$$
\begin{aligned}
& z_{l} \cos (\operatorname{Re}\{k\} x-t)+\varepsilon z_{l, 2} \cos (\operatorname{Re}\{k\} x-t) \text { and } \\
& z_{r} \cos (\operatorname{Re}\{k\} x+t-\theta)+\varepsilon z_{r, 2} \cos \left(\operatorname{Re}\{k\} x+t-\theta_{m}\right),
\end{aligned}
$$

with $z_{l}=z_{r}=z_{l, 2}=z_{r, 2}=0.7$ and $\theta_{2}=0$. In that case, the difference between the $\mathrm{M}_{4}$ amplitude of the idealized model and that of GETM decreases at the boundaries, but increases in the middle of the channel.

Figure 2.2 suggests that the amplitude of the sea surface elevation at the Vlie will be higher than at the Marsdiep. This agrees with data presented in the Royal Netherlands Navy, Hydrographic Service (2016) and the results of GETM also demonstrate this behavior (Figure 2.10b). In Section 2.5.1 it was shown that this amplitude difference is also possible without a difference in the external forcing. 


\subsection{Conclusions}

The first objective of this study was to assess changes in tidal characteristics of sea surface elevation and current velocity due to the momentum sink in double inlet systems. It is found that the momentum sink decreases the $\mathrm{M}_{2}$ amplitude of both the current velocity and the sea surface elevation and favours flood dominant tides. In a prototype system, viz. the Marsdiep-Vlie system in the Western Wadden Sea, it implies a decrease of $\mathrm{M}_{2}$ amplitudes of approximately $2 \%$ and an increase of both $\mathrm{M}_{4}$ amplitudes by $25 \%$. The absolute value of the residual sea surface elevation increased by $30 \%$ due to the momentum sink, whereas the residual current velocity was unaffected. The phases of the $\mathrm{M}_{2}$ tidal harmonic of both sea surface elevation and current velocity were not influenced by the momentum sink; only the $\mathrm{M}_{4}$ phases were lowered by approximately 10-15 degrees. In total, this amounts to an increase of net import of coarse sediment by approximately 35\%. Accumulation of coarse sediment was found inside the domain, indicating the formation of a tidal watershed. The transport of fine sediment was hardly influenced.

It is found that the changes in tidal characteristics are most sensitive to the flat-to-channel area ratio. The change in amplitude depends nonlinearly on that ratio; the sensitivity is smaller for larger values of the ratio. Furthermore, it is found that the momentum sinks affects the tidal harmonics of the sea surface elevation and current velocity more strongly when the drag coefficient is small and when the slope of the tidal flats decreases, at the right side of the channel $(0<x<L / 2)$, the sea surface elevation $\mathrm{M}_{4}$ amplitude does the same. A phase difference between the incoming tidal waves leads to spatial shifts of the curves that show amplitudes and phases versus along-chanel distance and to a small decrease of the $\mathrm{M}_{2}$ and $\mathrm{M}_{4}$ amplitudes. Finally, it is found that the difference in $\mathrm{M}_{2}$ sea surface elevation amplitude due to the momentum sink has a local maximum for a length of the embayment at approximately $45 \mathrm{~km}$.

The second objective was to understand the mechanisms behind the changes in tidal characteristics due to the momentum sink. The reduction of the $\mathrm{M}_{2}$ harmonic amplitude of the sea surface elevation and current velocity by the momentum sink is attributable to that, during the fall of the tide, still water enters the channel and slows down the current. The increase in $\mathrm{M}_{4}$ amplitude of the sea surface elevation and current velocity is explained by noting that the momentum sink acts as an advective term during the fall of the tide, but with a smaller amplitude. Since advection is known to favour flood currents (e.g. Friedrichs and Aubrey, 1988, Ridderinkhof et al., 2014), the momentum sink does so as well and increases the $\mathrm{M}_{4}$ amplitude in a flood dominant system.

A comparison with the complex numerical model GETM showed that the two models produce similar $\mathrm{M}_{2}$ amplitudes of the sea surface elevation. When the momentum sink is accounted for and an $\mathrm{M}_{4}$ harmonic is added to the incoming tidal waves, the amplitude of the $\mathrm{M}_{4}$ harmonic of the sea surface 
elevation shows a closer resemblance to the one modelled by GETM.

\subsection{Appendix}

\subsubsection{Derivation of term $M^{*}$ in equation (2.2.4)}

In this section, the expression for the momentum sink term $M^{*}$ in equation (2.2.4) is derived. The derivation only concerns dimensional equations and the asterisks are omitted to keep notation as simple as possible. Equations (2.2.3)-(2.2.4) follow from integrating the depth averaged shallow water equations over the channel width. The latter equations read

$$
\begin{aligned}
\frac{\partial \zeta}{\partial t}+\frac{\partial(\zeta+h) \bar{u}}{\partial x}+\frac{\partial(\zeta+h) \bar{v}}{\partial y} & =0 \\
\frac{\partial(\zeta+h) \bar{u}}{\partial t}+\frac{\partial(\zeta+h) \bar{u} \bar{u}}{\partial x}+\frac{\partial(\zeta+h) \bar{u} \bar{v}}{\partial y} & =-g(\zeta+h) \frac{\partial \zeta}{\partial x}-\frac{\bar{\tau}_{b}}{\rho},
\end{aligned}
$$

where, $\bar{u}\left(\mathrm{~m} \mathrm{~s}^{-1}\right)$ is the depth averaged longitudinal velocity, $\bar{v}\left(\mathrm{~m} \mathrm{~s}^{-1}\right)$ the depth averaged lateral velocity and $\bar{\tau}_{b}\left(\mathrm{~N} \mathrm{~m}^{-2}\right)$ the bottom stress. Note that $\zeta$ does not depend on $y$.

Integration of equation (2.7.2) over the width of the channel $\left[-b_{c} / 2, b_{c} / 2\right]$, using the continuity equation (2.2.3) and neglecting the stresses $(\bar{u}-u)(\bar{u}-u)$ arising from averaging over the width, yields

$$
\begin{aligned}
& b_{c}\left(\zeta+h_{c}\right) \frac{\partial u}{\partial t}+\underbrace{b_{c} u \frac{\partial \zeta}{\partial t}}_{A}-\underbrace{u b \frac{\partial \zeta}{\partial t}}_{B}+b_{c}\left(\zeta+h_{c}\right) u \frac{\partial u}{\partial x} \\
& +\underbrace{\int_{-b_{c} / 2}^{b_{c} / 2} \frac{\partial(\zeta+h) \bar{u} \bar{v}}{\partial y} d y}_{C}+b_{c} g\left(\zeta+h_{c}\right) \frac{\partial \zeta}{\partial x}+b_{c} \frac{\tau_{b}}{\rho}=0,
\end{aligned}
$$

in which $u$ (without the bar) represents the cross-sectionally averaged velocity. Term $A$ arises from integration of the first term in equation (2.7.2) over the width of the channel and term $B$ from integrating the second term in equation (2.7.2) and subsequently substituting the continuity equation (2.2.3). Term $C$ represents the lateral exchange of longitudinal momentum between the channel and the flats, and it equals

$$
C=\left.(\zeta+h) \bar{v} \bar{u}\right|_{y=b_{c} / 2}-\left.(\zeta+h) \bar{v} \bar{u}\right|_{y=-b_{c} / 2} .
$$

This expression is subsequently rewritten in terms of cross-sectionally averaged velocities. Integration of the continuity equation (2.7.1) over the right and left flats yields the volume transport (per unit length) through the boundary of the main channel,

$$
\left.(\zeta+h) \bar{v}\right|_{y= \pm b_{c} / 2}= \pm \frac{b-b_{c}}{2} \frac{\partial \zeta}{\partial t} .
$$


During the rising of the water level, $\bar{v}$ is positive at $y=b_{c} / 2$ and along-channel momentum is transferred towards the flats. When the water level falls, water moves from the flats to the main channel. Since the momentum is dissipated on the flats, no along-channel momentum returns to the channel. This motivates the choice

$$
\left.\bar{u}\right|_{y= \pm b_{c} / 2}=u \mathcal{H}\left(\frac{\partial \zeta}{\partial t}\right) .
$$

Substitution of equations (2.7.5)-(2.7.6) in (2.7.4) yields

$$
C=\left(b-b_{c}\right) u \mathcal{H}\left(\frac{\partial \zeta}{\partial t}\right) \frac{\partial \zeta}{\partial t} .
$$

The momentum sink term $M$ as in the main text is now obtained by combining terms $A, B$ and $C$, using $1-\mathcal{H}(x)=\mathcal{H}(-x)$ and dividing by $b_{c}\left(\zeta+h_{c}\right)$, which yields

$$
M=\frac{A+B+C}{b_{c}\left(\zeta+h_{c}\right)}=\frac{b-b_{c}}{b_{c}} \frac{\bar{u}}{\zeta+h_{c}} \mathcal{H}\left(-\frac{\partial \zeta}{\partial t}\right) \frac{\partial \zeta}{\partial t} .
$$

If the momentum sink is neglected $\left(\left.\bar{u}\right|_{y= \pm b_{c} / 2}=u\right)$, then $A+B=-C$ and hence $M=0$.

To frame equations $(2.2 .3)-(2.2 .5)$ in context of previous studies, note that Dronkers (1964) suggested that

$$
M=j_{1} \frac{b-b_{c}}{b_{c}} \frac{u}{\zeta+h_{c}} \frac{\partial \zeta}{\partial t}
$$

where $j_{1}=0$ when the water level rises and otherwise depends on the velocities on the flats. Speer and Aubrey (1985) and Friedrichs and Aubrey (1988) assumed the term $\left(b-b_{c}\right) / b_{c}$ to be small, and $M$ was therefore neglected. Speer (1984) also considered the case with $j_{1}=1$. In that case the tidal flats act as momentum storage regions. The choice made in Alebregtse (2015), de Swart et al. (2011) and the current study is $j_{1}=\mathcal{H}\left(-\frac{\partial \zeta}{\partial t}\right)$.

\subsubsection{Fourier coefficients of momentum sink term}

In the main text term $\mathrm{V}$ is written as

$$
\beta u_{0} \frac{\partial \zeta_{0}}{\partial t} \mathcal{H}\left(-\frac{\partial \zeta_{0}}{\partial t}\right)=\sum_{m=-\infty}^{\infty} p_{m} e^{-i m t} .
$$

The Fourier coefficients $p_{m}$ are

$$
p_{m}=\frac{i \beta}{4}\left(c_{2+m}^{*} \hat{u}_{0}^{*} \hat{\zeta}_{0}^{*}-c_{2-m} \hat{u}_{0} \hat{\zeta}_{0}\right)-\frac{\beta}{2} c_{m}^{*}\left|\hat{u}_{0}\right|\left|\hat{\zeta}_{0}\right| \sin \left(\phi_{\hat{u}_{0}}-\phi_{\hat{\zeta}_{0}}\right),
$$


where (in this appendix) $*^{*}$ denotes the complex conjugate and

$$
c_{m}= \begin{cases}\frac{1}{2} & \text { if } m=0 \\ 0 & \text { if } m \neq 0 \text { and even } \\ \frac{-i}{m \pi} e^{-i m \phi_{\hat{\zeta}_{0}}} & \text { if } m \text { odd }\end{cases}
$$

such that

$$
\mathcal{H}\left(-\frac{\partial \zeta_{0}}{\partial t}\right)=\sum_{m=-\infty}^{\infty} c_{m} e^{i m t} .
$$

In order to see that the phase of the $\mathrm{M}_{4}$ harmonic of term $\mathrm{V}$ equals that of the advection term, note that term $\mathrm{V}$ can be written as a product of a constant, the advection term and the step function as in equation (2.5.1). From equations (2.3.10)-(2.3.14) it follows that the advection term consists of an $\mathrm{M}_{0}$ and $\mathrm{M}_{4}$ harmonic,

$$
u_{0} \frac{\partial u_{0}}{\partial x}=A_{M_{0}}+\operatorname{Re}\left\{A_{M_{4}} e^{-2 i t}\right\},
$$

where $A_{M_{0}}$ is a real number and $A_{M_{4}}$ a complex number. Since the even Fourier coefficients of the step function $c_{m}$ are zero, multiplying (2.7.9) and (2.7.8) yields that the $\mathrm{M}_{4}$ harmonic of the product equals

$$
c_{0} \operatorname{Re}\left\{A_{M_{4}} e^{-2 i t}\right\} .
$$

Hence, the phase of the $\mathrm{M}_{4}$ harmonic of term $\mathrm{V}$ equals $\arg \left(A_{M_{4}}\right)$ and thus the phase of the $\mathrm{M}_{4}$ harmonic of the advection term. 
Chapter 2. Momentum dissipation on tidal flats 


\section{3}

\section{Sensitivity of tidal bar wavelength to channel width}

T. M. Hepkema, H.E. de Swart and H. M. Schuttelaars.

Sensitivity of tidal bar wavelength to channel width. Journal of Geophysical Research: Earth Surface, 124, 2417-2436 (2019). https://doi.org/10.1029/2019JF005032 


\begin{abstract}
Tidal bars are repetitive estuarine bedforms with heights of several meters and wavelengths in the order $1-15 \mathrm{~km}$. Understanding their formation and sensitivity to changes in channel characteristics is important as they hamper marine traffic and play a crucial role in the local ecosystem. Recent observations suggest that the local width of the channel is dominant in determining the tidal bar wavelength. However, theoretical studies could not reproduce the sensitivity of the tidal bar wavelength to channel width. This discrepancy between theory and observations suggests that a mechanism is missing. In this study, one of the theoretical models is extended and results in tidal bar wavelengths, lateral mode numbers and growth rates that agree fairly well with those of natural tidal bars, including the wavelengths dependency on estuary width. An important extension of the model concerns the bed slope induced diffusive suspended load transport of sediments. With this, it is explained why previously the modelled tidal bar wavelengths depend only weakly on estuary width and why in the extended model it does. This has, from a modelling point of view, general implications for morphological models using a total load sediment transport formulation with a so-called bed slope parameter.
\end{abstract}




\subsection{Introduction}

Many tidal channels have a rhythmic bottom topography consisting of so-called tidal bars. Examples are the Ord River estuary in Australia, the Exe estuary in England, the Netarts bay in the USA and the Western Scheldt in the Netherlands (see Figure 3.1 and Leuven et al. (2016) for many more examples). These bars have wavelengths of $1-15 \mathrm{~km}$ and heights of several meters. As tidal bars hamper marine traffic and provide rich feeding grounds for many living organisms, it is important to understand their formation and sensitivity to tidal channel characteristics (i.e., channel width, depth, currents, etc.). These characteristics may change due to e.g., dredging, sea level rise or land reclamation. The sensitivity of the tidal bars to changes in channel characteristics was previously investigated by measurements, laboratory experiments, idealized models and complex numerical models.

By analyzing data of 25 tidal bars in creeks in South Carolina and in the Salmon River estuary, Dalrymple and Rhodes (1995) found that the tidal bar wavelength is typically six times the channel width. Also, Leuven et al. (2016) found that the tidal bar wavelength correlates the strongest with channel width, compared to other channel characteristics such as depth and current velocity. They came to this conclusion by measuring the wavelength of 190 tidal bars in Google Earth. Furthermore, Tambroni et al. (2005) investigated morphodynamic equilibria in tidal channels and inlets in a laboratory. Their tidal bars formed after 50 tidal cycles with wavelengths of approximately three times the channel width.

To gain fundamental understanding about tidal bars, Seminara and Tubino (2001) analysed a 3D idealized model. In their model the section of the channel, in which the tidal bars are studied, is assumed to be short with respect to the tidal wavelength, width variations and depth variations. This setting differs from, for example, Schuttelaars and de Swart (1999) and ter Brake and Schuttelaars (2011), where the formation of tidal bars in a short semi-enclosed channel was investigated. Seminara and Tubino (2001) demonstrated that tidal bars can form as a free instability of an equilibrium state that describes a horizontally uniform tidal current over a horizontal bed. They found that the wavelengths of their modelled bars are comparable with those of observed bars. It further follows from their results that the ratio of channel width over wavelength of the bars scales close to linearly with the width-to-depth ratio. This implies that for a constant depth, the wavelength hardly depends on channel width. Schramkowski et al. (2002) showed that a depth-averaged model is able to model characteristics of tidal bars and that a 3D model is not needed. They included the local inertia terms in the momentum balance and showed that the current velocity and the water depth are also important in determining the tidal bar wavelength. This model also yields a tidal bar wavelength that only depends weakly on channel width (Leuven et al., 2016). Garotta et al. (2006) 
confirmed that a 2D model is sufficient and moreover showed that forcing by a tidal current that is either ebb or flood dominant leads to a net migration of bars.

Thus, the idealized models are not able to reproduce the observed sensitivity of tidal bar length on channel width. This could be a consequence of the many assumptions made in these models. To clarify this, numerical models, which contain less constraints, are helpful. Such a study was carried out by Hibma et al. (2004). Their numerical experiments were designed such that comparison with the idealized studies was possible. They found tidal bar wavelengths that are comparable with those found by Schramkowski et al. (2002). However, despite expectations after the work of Schramkowski et al. (2002), the wavelength was, besides sensitive to current velocity and channel depth, also sensitive to channel width. Yet, they did not investigate this further. Also, van der Wegen and Roelvink (2008) found that the wavelength depends on both the estuary width, depth and the current velocity. In addition, van de Lageweg and Feldman (2018) found that, in a strongly convergent estuary, an increase in basin width results in longer bars.

The considerations above show in particular that the idealized models are not able to explain the observed (and numerically reproduced) dependence of tidal bar length on channel width. A likely explanation for this discrepancy is that one or more essential physical processes are missing in these models. Compared with numerical model formulations, it turns out that the idealized models do not account for the effects of turbulent exchange of momentum and sediment in the horizontal plane. The intensities of these processes are measured by a horizontal eddy viscosity coefficient and a horizontal eddy diffusion coefficient, respectively. Therefore, in the present study the model of Schramkowski et al. (2002) is extended with terms that describe horizontal turbulent exchange processes. Moreover, a critical current velocity for sediment erosion is added to achieve consistency with all other models.

The first aim of this work is to show that the extended model can mimic the pattern of observed tidal bars. The second aim is to show that the model can reproduce the sensitivity of tidal bar wavelength to channel width. The first and second aim are treated in Section 3.3. Finally, we aim at explaining why the incorporation of the new processes results in a dependence of the tidal bar wavelength to channel width (Section 3.4).

\subsection{Model and method}

\subsubsection{Governing equations}

The formation of tidal bars is modelled in a section of a tidal channel which is short compared to both the tidal wavelength and the $e$-folding length scale of channel width convergence. The model domain under consideration therefore 
consists of a 2D rectangular channel of fixed width $B$, mean depth $H$ and infinite length (Figure 3.2). Note that by channel width $B$ the total width of the channel is meant (see also Figure 3.1). The hydrodynamics is modelled by the depth-averaged shallow water equations including horizontal eddy viscosity and assuming the rigid-lid approximation. The latter constitutes to only retaining the free surface elevation in the pressure gradient. Coriolis effects are not considered and the bed shear stress and internal stresses are linearized. The depth-integrated sediment concentration is modelled by an advection-diffusion equation based on ter Brake and Schuttelaars (2010) and extended by including a critical velocity for erosion. The bed evolution (Exner) equation follows from the conservation of sediment mass. Since the convergence of sediment transport is almost periodic (with a period equal to the principal tidal constituent), the subtidal convergence is small compared to the instantaneous convergence of sediment transport. Therefore, the patterns evolve on a much larger time scale than the tidal period and is determined by the tidally averaged convergence of sediment transport (see Krol (1991) for a discussion on the errors introduced by this approximation). The differences with the model of Schramkowski et al. (2002) are the critical velocity for erosion, the horizontal eddy viscosity and

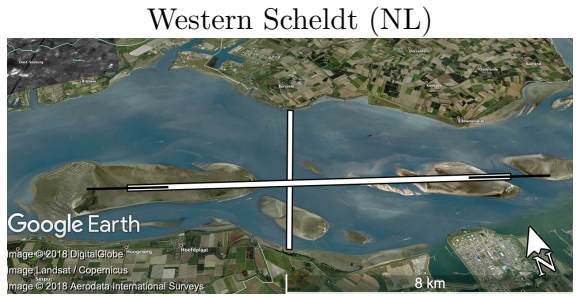

Exe estuary (UK)

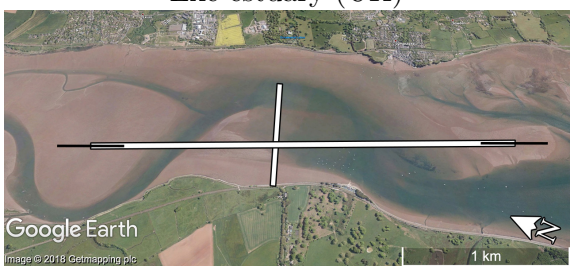

Ord River estuary (AU)

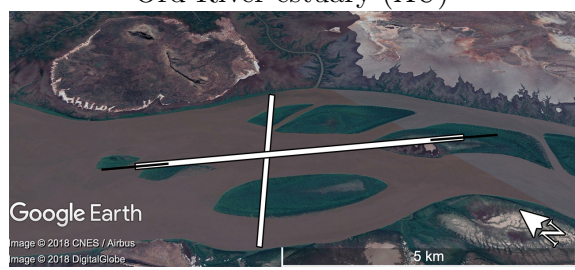

Netarts bay (USA)

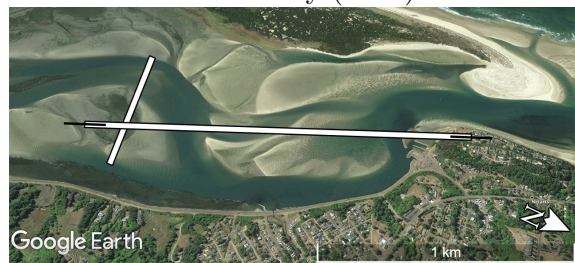

Figure 3.1: Measured tidal bar wavelength and channel width of four tidal channels in Google Earth. 

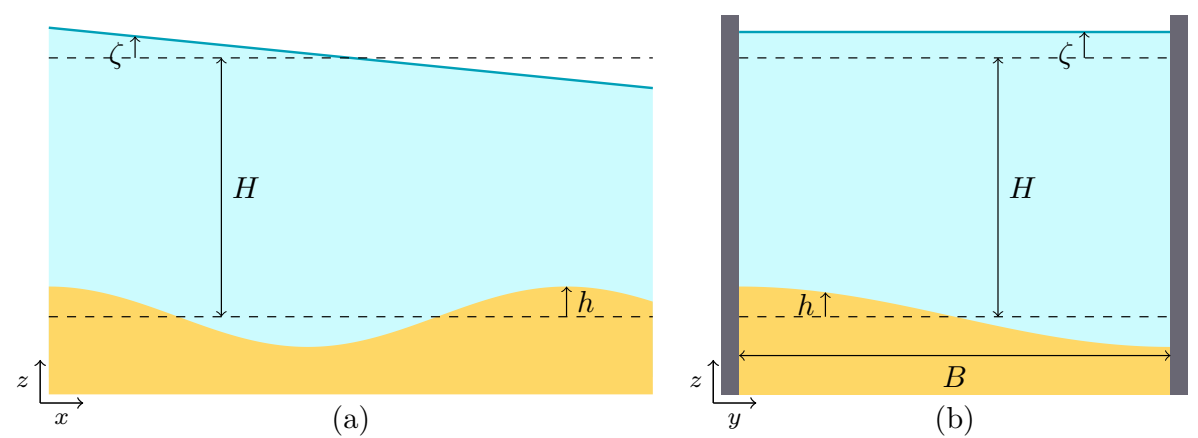

Figure 3.2: (a) Along-channel section in $(x, z)$-plane. (b) Cross-channel section in $(y, z)$-plane. $H$ denotes the undisturbed depth, $\zeta$ the free surface elevation, $h$ the bottom height and $B$ the channel width. The arrows with $x, y$ and $z$ denote the direction of the spatial coordinates.

horizontal eddy diffusivity. The resulting governing equations read

$$
\begin{aligned}
\boldsymbol{\nabla} \cdot((H-h) \boldsymbol{u}) & =0 \\
\frac{\partial \boldsymbol{u}}{\partial t}+(\boldsymbol{u} \cdot \boldsymbol{\nabla}) \boldsymbol{u}+\frac{r \boldsymbol{u}}{H-h}-\nu \nabla^{2} \boldsymbol{u} & =\boldsymbol{F}_{\boldsymbol{p}} \\
\frac{\partial C}{\partial t}+\nabla \cdot(\boldsymbol{u} C)-\boldsymbol{\nabla} \cdot\left(\mu \boldsymbol{\nabla} C+\mu \frac{w_{s}}{\kappa_{v}} \beta_{b} C \boldsymbol{\nabla} h\right) & =E-D, \\
(1-\hat{p}) \frac{\partial h}{\partial t}+\boldsymbol{\nabla} \cdot\left\langle\boldsymbol{q}_{\mathrm{s}}+\boldsymbol{q}_{\mathrm{b}}\right\rangle & =0 .
\end{aligned}
$$

The space and time coordinates are $\boldsymbol{x}=(x, y)[\mathrm{m}]$ and $t[\mathrm{~s}]$, respectively. Furthermore, $\boldsymbol{u}(t, \boldsymbol{x})=(u, v)\left[\mathrm{m} \mathrm{s}^{-1}\right]$ is the depth-averaged flow velocity vector, $C(t, \boldsymbol{x})\left[\mathrm{m}^{3} \mathrm{~m}^{-2}\right]$ the depth-integrated volumetric sediment concentration and $h(t, \boldsymbol{x})[\mathrm{m}]$ the height of the bottom with respect to a flat undisturbed bottom (see Fig. 3.2). $H[\mathrm{~m}]$ is the undisturbed water depth, $r\left[\mathrm{~m} \mathrm{~s}^{-1}\right]$ a friction coefficient and $\nu\left[\mathrm{m}^{2} \mathrm{~s}^{-1}\right]$ the horizontal eddy viscosity coefficient. Moreover, $\mu\left[\mathrm{m}^{2} \mathrm{~s}^{-1}\right]$ is the horizontal eddy diffusion coefficient, $w_{s}\left[\mathrm{~m} \mathrm{~s}^{-1}\right]$ the sediment settling velocity, $\kappa_{v}\left[\mathrm{~m}^{2} \mathrm{~s}^{-1}\right]$ the vertical eddy diffusivity coefficient, $\beta_{b}$ a deposition parameter and $\hat{p}$ the porosity parameter. The prescribed pressure gradient force per mass unit $\boldsymbol{F}_{p}=-g \boldsymbol{\nabla} \zeta\left[\mathrm{m} \mathrm{s}^{-2}\right]$, with $g\left[\mathrm{~m} \mathrm{~s}^{-2}\right]$ the gravitational acceleration and $\zeta(t, \boldsymbol{x})[\mathrm{m}]$ the free surface elevation, drives a tidal current in the channel of which the dominant constituent has radian frequency $\sigma\left[\mathrm{s}^{-1}\right]$ and a typical amplitude $U$. Furthermore, $\langle\cdot\rangle$ is the average over one tidal cycle with period $2 \pi / \sigma$. Erosion $E$ and deposition $D$ are defined as

$$
E=\alpha \mathcal{H}\left(\|\boldsymbol{u}\|^{2}-u_{c}^{2}\right)\left(\|\boldsymbol{u}\|^{2}-u_{c}^{2}\right) \quad \text { and } \quad D=\gamma C,
$$

with $\mathcal{H}$ the Heaviside function, which is zero (one) when its argument is (not) negative and $\|\cdot\|$ denotes the Euclidean norm of a vector. In these expressions, 
$\alpha\left[\mathrm{s} \mathrm{m}^{-1}\right]$ is an erosion parameter, $u_{c}\left[\mathrm{~m} \mathrm{~s}^{-1}\right]$ the critical velocity for erosion and $\gamma\left[\mathrm{s}^{-1}\right]$ a deposition parameter. Lastly, $\boldsymbol{q}_{\mathrm{s}}\left[\mathrm{m}^{2} \mathrm{~s}^{-1}\right]$ is the transport of suspended sediment and $\boldsymbol{q}_{\mathrm{b}}\left[\mathrm{m}^{2} \mathrm{~s}^{-1}\right]$ the transport of sediment as bed load. In Schramkowski et al. (2002) it is argued that the bed load transport is much smaller than the suspended load transport but that the bed slope contribution to the bed load transport should be maintained. Here, the suspended load transport is extended by a diffusivity term based on ter Brake and Schuttelaars (2010). The sediment transport formulations read

$$
\boldsymbol{q}_{\mathrm{s}}=\boldsymbol{u} C-\mu\left(\boldsymbol{\nabla} C+\frac{w_{s}}{\kappa_{v}} \beta_{b} C \boldsymbol{\nabla} h\right) \quad \text { and } \quad \boldsymbol{q}_{\mathrm{b}}=-\hat{s} \kappa_{\star}\|\boldsymbol{u}\|^{3} \nabla h,
$$

where $\hat{s}\left[\mathrm{~s}^{2} \mathrm{~m}^{-1}\right]$ is a bed load transport constant and $\kappa_{\star}$ a bed slope parameter.

The bottom friction coefficient $r=8 /(3 \pi) c_{d} \mathcal{U}$ is chosen to scale with a typical current velocity amplitude. The horizontal eddy diffusivity coefficients $\nu=c_{h}^{u} \mathcal{U} B$ and $\mu=c_{h}^{c} \mathcal{U} B$ scale with current velocity and the channel width. The vertical eddy diffusivity $\kappa_{v}=c_{v} \mathcal{U} H$ scales with current velocity and the undisturbed channel depth. Here, $c_{h}^{c}, c_{h}^{u}, c_{v}$ are constants and $\mathcal{U}$ is a typically velocity, which will be specified in the next section. All parameters are summarized in Table 3.1. The bed load transport formulation and constants $\hat{s}$ and $\kappa_{\star}$ are taken from Schramkowski et al. (2002) and based on Bailard (1981). The value of the erosion parameter $\alpha$ is also taken from Schramkowski et al. (2002) and based on Smith and McLean (1977), the deposition parameters $\gamma$ and $\beta_{b}$ are based on the derivation in ter Brake and Schuttelaars (2010) and the settling velocity $w_{s}$ corresponds to sediment with a median grain diameter $d_{50}=0.15 \mathrm{~mm}$. For the reasoning behind the values of the sediment parameters we refer to the books of Dyer (1986) and Soulsby (1997).

The lateral boundary conditions imposed are

$$
v=0, \quad \frac{\partial C}{\partial y}=0, \quad \frac{\partial h}{\partial y}=0 \quad \text { and } \quad \frac{\partial u}{\partial y}=0 \quad \text { at } y=0, B .
$$

This means that there is neither transport of water nor sediment through the solid boundaries of the channel. Furthermore, all variables are assumed periodic in the longitudinal direction. Further details and underlying assumptions of the model are given in Schramkowski et al. (2002).

\subsubsection{Linear stability analysis}

Similar to the system in Schramkowski et al. (2002), the system (3.2.1)-(3.2.4) admits a spatially uniform equilibrium solution $\left(\left(\boldsymbol{F}_{\boldsymbol{p}}\right)_{\text {eq }}, \boldsymbol{u}_{\text {eq }}, C_{\text {eq }}, h_{\text {eq }}\right)$, which is here chosen to be an $\mathrm{M}_{2}$ tidal flow

$$
\boldsymbol{u}_{\mathrm{eq}}=\left(u_{\mathrm{eq}}, v_{\mathrm{eq}}\right)=(U \cos (\sigma t), 0)
$$

over a flat bottom $h_{\mathrm{eq}}=0$, with $U\left[\mathrm{~ms}^{-1}\right]$ the constant amplitude of the longitudinal equilibrium velocity $u_{\mathrm{eq}}$. The corresponding pressure gradient 


\begin{tabular}{|c|c|c|c|}
\hline Parameter & value(s) & units & name \\
\hline$H$ & $1.2-10$ & $\mathrm{~m}$ & undisturbed water depth \\
\hline$B$ & $800-7000$ & $\mathrm{~m}$ & channel width \\
\hline$U$ & $0.6-1$ & $\mathrm{~m} \mathrm{~s}^{-1}$ & amplitude equilibrium velocity \\
\hline$r=\frac{8}{3 \pi} c_{d} \mathcal{U}$ & & $\mathrm{ms}^{-1}$ & friction coefficient \\
\hline$\kappa_{v}=c_{v} \mathcal{U} H$ & & $\mathrm{~m}^{2} \mathrm{~s}^{-1}$ & vertical eddy diffusivity coefficient \\
\hline$\nu=c_{h}^{u} \mathcal{U} B$ & & $\mathrm{~m}^{2} \mathrm{~s}^{-1}$ & horizontal eddy viscosity coefficient \\
\hline $\begin{array}{l}\mu=c_{h}^{c} \mathcal{U} B \\
\mathcal{U}=U\end{array}$ & & $\begin{array}{l}\mathrm{m}^{2} \mathrm{~s}^{-1} \\
\mathrm{~m} \mathrm{~s}^{-1}\end{array}$ & $\begin{array}{l}\text { horizontal eddy diffusion coefficient } \\
\text { typical current velocity }\end{array}$ \\
\hline$\gamma=\frac{w_{s}^{2}}{s} \beta_{b}$ & & $\mathrm{~s}^{-1}$ & deposition parameter \\
\hline$\Lambda=\kappa_{\star} \hat{s}\left\langle\left|u_{e q}^{3}\right|\right\rangle$ & & $\mathrm{m}^{2} \mathrm{~s}^{-1}$ & bed load bed slope parameter \\
\hline$\left.\beta_{b}=\left(1-e^{\frac{-w_{s}}{\kappa_{v}} H}\right)\right)^{-1}$ & & & deposition parameter \\
\hline$g$ & 9.81 & $\mathrm{~ms}^{-2}$ & gravitational acceleration \\
\hline$\sigma$ & $1.4 \cdot 10^{-4}$ & $\mathrm{~s}^{-1}$ & $\mathrm{M}_{2}$ tidal frequency \\
\hline$w_{s}$ & 0.013 & $\mathrm{~ms} \mathrm{~s}^{-1}$ & settling velocity \\
\hline$\alpha$ & $5 \cdot 10^{-6}$ & $\mathrm{~s} \mathrm{~m}^{-1}$ & erosion parameter \\
\hline$u_{c}$ & $0-0.3$ & $\mathrm{~ms}^{-1}$ & critical velocity for erosion \\
\hline$\hat{s}$ & $3 \cdot 10^{-4}$ & $\mathrm{~s}^{2} \mathrm{~m}^{-1}$ & bed load transport constant \\
\hline$\hat{p}$ & 0.4 & & porosity parameter \\
\hline$c_{d}$ & 0.0025 & & drag coefficient \\
\hline$\kappa_{\star}$ & 2 & & bed load bed slope parameter \\
\hline$c_{v}$ & 0.001 & & vertical diffusivity constant \\
\hline$c_{h}^{c}$ & $0-0.0035$ & & horizontal diffusivity constant \\
\hline$c_{h}^{n}$ & $0-0.001$ & & horizontal viscosity constant \\
\hline
\end{tabular}

Table 3.1: Parameters and their value (range) after ter Brake and Schuttelaars (2010) and Schramkowski et al. (2002).

$\left(\boldsymbol{F}_{\boldsymbol{p}}\right)_{\mathrm{eq}}=-g \boldsymbol{\nabla} \zeta_{\mathrm{eq}}$ and sediment concentration $C_{\text {eq }}$ are given in Appendix 3.6.1. The typical current velocity scale $\mathcal{U}$ is chosen to be the amplitude of the equilibrium velocity $U$. Other harmonic compositions can be added to $u_{\text {eq }}$, but are not considered here.

Tidal bars form as an instability on this spatially uniform equilibrium,

$$
\left(\left(\boldsymbol{F}_{\boldsymbol{p}}\right)_{\mathrm{eq}}, \boldsymbol{u}_{\mathrm{eq}}, C_{\mathrm{eq}}, h_{\mathrm{eq}}\right) .
$$

Perturbations in the bottom $h_{\mathrm{eq}}+h^{\prime}$, result in perturbations in the flow $\boldsymbol{u}_{\mathrm{eq}}+\boldsymbol{u}^{\prime}$, the pressure gradient $-g\left(\nabla \zeta_{\mathrm{eq}}+\nabla \zeta^{\prime}\right)$ and the sediment concentration $C_{\mathrm{eq}}+C^{\prime}$. These perturbations in turn, result in positive or negative feedbacks resulting in growth or decay of the bottom perturbations $h^{\prime}$. The pattern with the largest growth overtakes the others and yields the length and time scale of the pattern that is formed initially (Dodd et al., 2003).

Substituting $\zeta=\zeta_{\mathrm{eq}}+\zeta^{\prime}, u=u_{\mathrm{eq}}+u^{\prime}, v=v_{\mathrm{eq}}+v^{\prime}, C=C_{\mathrm{eq}}+C^{\prime}$ and $h=h_{\mathrm{eq}}+h^{\prime}$, in equations (3.2.1)-(3.2.4) and linearizing the resulting equations, yields a system of linear partial differential equations in the primed variables, which are perturbations on the equilibrium variables. The linearized equations are given in Appendix 3.6.2. This system of equations, together 
with the boundary conditions (3.2.5) in terms of the primed variables allow for solutions, which are linear combinations of

$$
\left(\begin{array}{c}
\zeta^{\prime} \\
u^{\prime} \\
v^{\prime} \\
C^{\prime} \\
h^{\prime}
\end{array}\right)=\operatorname{Re}\left\{\left(\begin{array}{c}
\hat{\zeta}(t) \cos \left(l_{n} y\right) \\
\hat{u}(t) \cos \left(l_{n} y\right) \\
\hat{v}(t) \sin \left(l_{n} y\right) \\
\hat{C}(t) \cos \left(l_{n} y\right) \\
\hat{h}(t) \cos \left(l_{n} y\right)
\end{array}\right) e^{i k x}\right\}
$$

for every natural number $n$ and real number $k>0$. Here, $l_{n}=n \pi / B$ and $\hat{\zeta}, \hat{u}, \hat{v}, \hat{C}$ and $\hat{h}$ are complex valued functions of time. The number $n$ is henceforth referred to as the mode number and is related to the number of bars and troughs in the lateral direction of the channel. For example, a pattern with mode number $n=1$ corresponds to an alternating bar pattern and the pattern with $n=2$ has one bar or a trough in the middle of the channel.

Since the perturbations in the bottom vary on a time scale that is much longer than the time scale on which perturbations of the other variables vary, the bottom is assumed to be constant during one tidal cycle. That is, $\hat{h}$ only depends on a long morphodynamic time scale while $\hat{\zeta}, \hat{u}, \hat{v}, \hat{C}$ depend on the short hydrodynamical time scale. This allows for calculating the fast variables $\hat{\zeta}, \hat{u}, \hat{v}$ and $\hat{C}$, assuming a fixed bottom. The so-called 'flow over topography problem' is solved during one tidal cycle.

Substituting equation (3.2.6) in the linearized continuity, momentum and concentration equation for a constant $\hat{h}$, results in a system of ordinary differential equations for $\hat{\zeta}, \hat{u}, \hat{v}$ and $\hat{C}$. The solution to this system of equations is approximated by a truncated Fourier series. This Fourier series contains the residual component and overtides with frequencies $p \sigma$, with $p$ an integer. Because the equations are linear and inhomogeneous, the resulting $\hat{\zeta}, \hat{u}, \hat{v}$ and $\hat{C}$ will be linear in the amplitude of the bed perturbations $\hat{h}$. Taking this into account, the amplitudes are approximated by choosing a large enough natural number $N$ and substituting

$$
(\hat{\zeta}, \hat{u}, \hat{v}, \hat{C})=\hat{h} \sum_{p=-N}^{N}\left(\tilde{\zeta}_{p}, \tilde{u}_{p}, \tilde{v}_{p}, \tilde{C}_{p}\right) e^{i p \sigma t}
$$

in the ordinary differential equations. Here, $\tilde{\zeta}_{p}, \tilde{u}_{p}, \tilde{v}_{p}$ and $\tilde{C}_{p}$ for $p=-N, \ldots, N$ are complex amplitudes, independent of $\hat{h}$. This results in a system of $4(2 N+1)$ algebraic equations in $\tilde{\zeta}_{p}, \tilde{u}_{p}, \tilde{v}_{p}$ and $\tilde{C}_{p}$, which can be solved numerically. Recall that, since the resulting velocity and concentration field is solved for a fixed $h^{\prime}$, the coefficients $\tilde{\zeta}_{p}, \tilde{u}_{p}, \tilde{v}_{p}$ and $\tilde{C}_{p}$ depend on $n$ and $k$. That is to say, the obtained $\left(\zeta^{\prime}, u^{\prime}, v^{\prime}, C^{\prime}\right)$ describe how the flow, the pressure gradient and the concentration react to the bottom perturbations $h^{\prime}=\operatorname{Re}\left\{\hat{h} \cos \left(l_{n} y\right) e^{i k x}\right\}$.

The next step is to determine the bed evolution. The linearized Exner 
equation reads

$$
(1-\hat{p}) \frac{\partial h^{\prime}}{\partial t}+\nabla \cdot\left\langle\boldsymbol{q}_{\mathrm{s}}^{\prime}+\boldsymbol{q}_{\mathrm{b}}^{\prime}\right\rangle=0
$$

with

$$
\boldsymbol{q}_{\mathrm{s}}^{\prime}=\boldsymbol{u}^{\prime} C_{\mathrm{eq}}+\boldsymbol{u}_{\mathrm{eq}} C^{\prime}-\mu\left(\nabla C^{\prime}+\frac{w_{s}}{\kappa_{v}} \beta_{b} C_{\mathrm{eq}} \nabla h^{\prime}\right) \quad \text { and } \quad \boldsymbol{q}_{\mathrm{b}}^{\prime}=-\Lambda \nabla h^{\prime},
$$

where $\Lambda=\hat{s} \kappa_{\star}\left\langle\left|u_{e q}^{3}\right|\right\rangle$. Since $\zeta^{\prime}, u^{\prime}, v^{\prime}, C^{\prime}$ are explicitly calculated in terms of $h^{\prime}$, substituting these expressions in equation (3.2.8) results in a single equation for $h^{\prime}$ and hence for $\hat{h}$,

$$
\frac{\partial \hat{h}}{\partial t}=\omega \hat{h}
$$

with complex growth rate

$$
\begin{aligned}
\omega=\frac{-1}{1-\hat{p}}( & \frac{i k U}{2}\left(\tilde{C}_{1}+\tilde{C}_{-1}\right)+\mu\left(k^{2}+l_{n}^{2}\right) \tilde{C}_{0}+\mu \frac{w_{s}}{\kappa_{v}} \beta_{b} \tilde{C}_{\mathrm{eq}, 0}\left(k^{2}+l_{n}^{2}\right) \\
& \left.+\Lambda\left(k^{2}+l_{n}^{2}\right)\right)
\end{aligned}
$$

in which the variables with the tildes are defined in equation (3.2.7) and $\tilde{C}_{\text {eq, } 0}$ in Appendix 3.6.1. The real part of the complex growth rate represents the actual growth rate of the bottom perturbation and $-\operatorname{Im}\{\omega\} / k$ its migration speed. Here, $\omega$ turns out to be real because $u_{\text {eq }}$ consists of one tidal constituent.

The preferred wavenumber and mode number $k_{\text {pref }}$ and $n_{\text {pref }}$ are defined as those for which the growth rate $\omega$ is maximal,

$$
\omega\left(k_{\text {pref }}, n_{\text {pref }}\right)=\max _{k, n}\{\omega(k, n)\} .
$$

The preferred growth rate $\omega_{\text {pref }}=\omega\left(k_{\text {pref }}, n_{\text {pref }}\right)$ and the preferred wavelength $\lambda_{\text {pref }}=2 \pi / k_{\text {pref }}$ is the wavelength that can be compared to the distance between natural tidal bars. The preferred wavenumber, mode number and growth rate are found using the optimization method 'Brent' (Press et al., 2007).

\subsection{Results}

\subsubsection{Verification}

The first objective is to verify that the model produces preferred wavelengths $\lambda_{\text {pref }}$, mode numbers $n_{\text {pref }}$ and $e$-folding growth time $1 / \omega_{\text {pref }}$ that resemble those of bars that are observed in nature. For this we selected four natural tidal channels. These four are chosen because they differ in width, depth and current 
velocities and roughly satisfy the model assumptions. Table 3.2 summarizes the geometric and current characteristics of these channels, as well as the observed wavelength $\lambda_{\text {pref }}^{M}$ and mode number $n_{\text {pref }}^{M}$ of the tidal bars. The wavelength, mode number and channel width are obtained from Google Earth (Figure 3.1). This is done by identifying the pattern of the form $\cos (n \pi y / B) \cos (k x)$ (as in equation (3.2.6)), which represent the observed channels in Google Earth most accurately. The wavelength $\lambda_{\text {pref }}^{M}$ is measured as the distance between successive crests. The uncertainty in the wavelength results from the uncertainty of the location of the crests of the bars. An alternating bar pattern $\left(n_{\text {pref }}^{M}=1\right)$ represents the patterns in the Exe estuary and the Netarts bay best. The patterns in the Ord and the Western Scheldt are described by patterns with a mode number close to three. The width of the pattern that fits best is as illustrated in Figure 3.1.

The currents and undisturbed depths of the Western Scheldt, Ord River estuary, Exe estuary and Netarts bay are based on Cancino and Neves (1999), Wolanski et al. (2001), Herrmann and Hübner (1982) and Glanzmann et al. (1971), respectively. Furthermore, these papers report that the considered channels are vertically well-mixed (i.e., small vertical salinity gradients) and the tides predominantly semi-diurnal with free surface elevation amplitudes of 1-2 m. The sediment properties are assumed to be similar in all channels (following Leuven et al., 2016). Typical time scales at which these bars evolve are of the order of decades.

The left column of Figure 3.3 shows the growth curves (i.e., graphs of $\omega(k, n))$ for the four different channels. The red vertical line represents the measured wavenumber and the red area the measurement uncertainty. The right column shows the bottom pattern corresponding to the preferred wavenumber and mode number on which the residual current is shown with arrows. The figure reveals that, using realistic parameter values, the model yields wavelengths of tidal bars that are of the same order of magnitude as those of bars observed in nature. Also, the preferred mode numbers $n_{\text {pref }}$ are in the right order of magnitude. They agree for the Exe estuary and the Netarts bay and for the Western Scheldt and the Ord River estuary the difference is one. Note that for the Ord River estuary, the maximal growth rates for the different mode numbers $n=1, n=2$ and $n=3$ are similar. Lastly, the $e$-folding growth time is in the order of decades.

\subsubsection{Sensitivity of tidal bar wavelength to channel width}

The second objective is to show that the extended model reproduces the sensitivity of the preferred wavelength to channel width. The lower lines in the top panels of Figure 3.4 (indicated by $c_{h}^{c}=0$ ) show the relation between the preferred wavelength $\lambda_{\text {pref }}$ and the channel width $B$ for the four channels when the horizontal eddy diffusivity $\mu$, horizontal eddy viscosity $\nu$ and the critical velocity for erosion $u_{c}$ are all zero. This case corresponds to the original model 

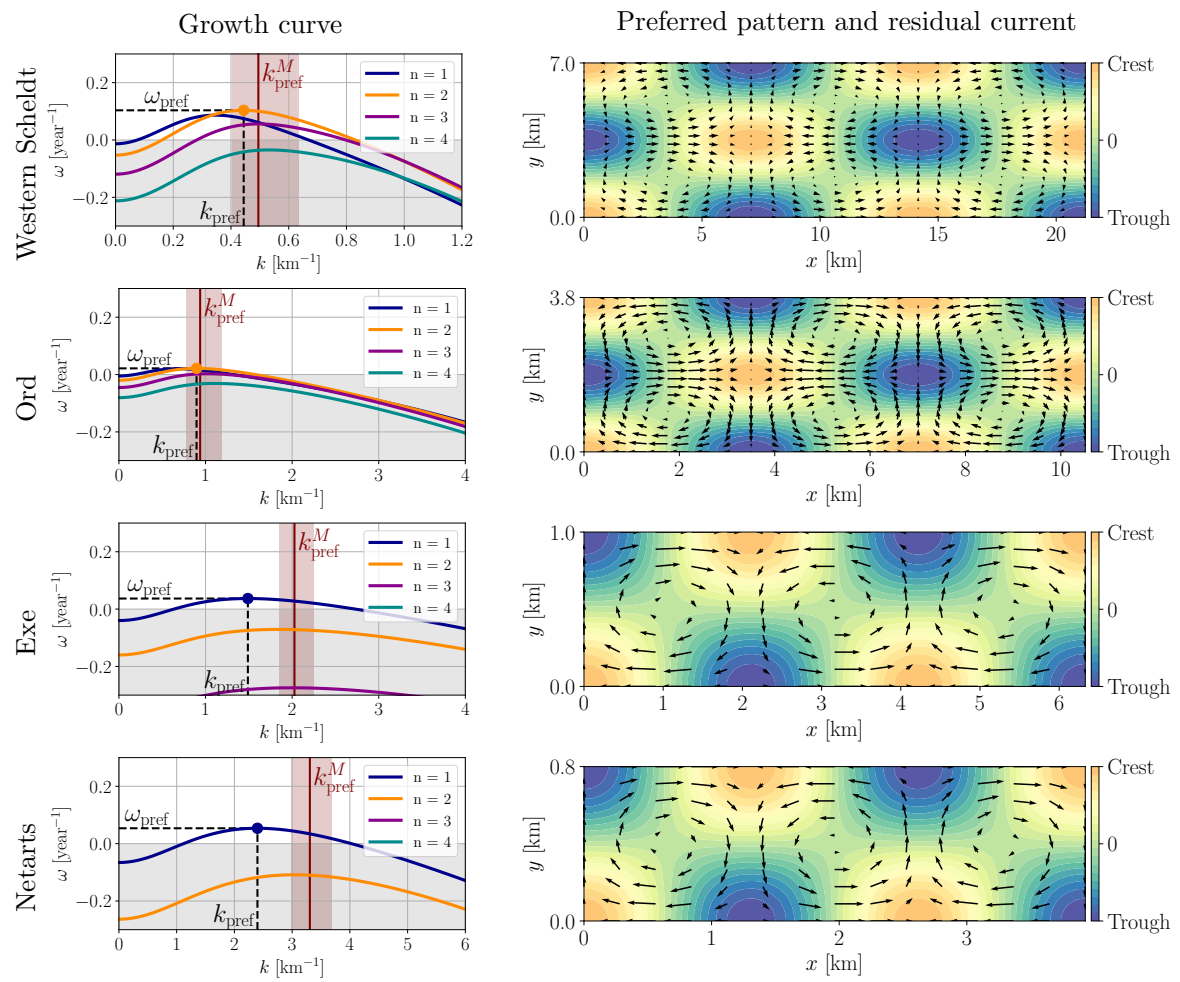

Figure 3.3: Left: Growth rate $\omega$ versus wavenumber $k$ for different mode numbers $n$ and four different channels (rows). The red vertical lines denote the measured wavenumber $k_{\mathrm{pref}}^{M}=2 \pi / \lambda_{\text {pref }}^{M}$ as in Table 3.2 and the red area the measurement uncertainty. Right: The preferred bottom pattern versus space for each channel. The arrows denote the residual current. Parameters are as in Table 3.1 and 3.2 with $c_{h}^{u}=c_{h}^{c}=0.001$ and $u_{c}=0.3 \mathrm{~m} \mathrm{~s}^{-1}$.

\begin{tabular}{lccccc}
\hline & $B[\mathrm{~km}]$ & $H[\mathrm{~m}]$ & $U\left[\mathrm{~m} \mathrm{~s}^{-1}\right]$ & $\lambda_{\text {pref }}^{M}[\mathrm{~km}]$ & $n_{\text {pref }}^{M}$ \\
\hline Western Scheldt & 7.0 & 10.0 & 1.0 & $12.7 \pm 2.8$ & 3 \\
Ord River estuary & 3.8 & 4.0 & 0.6 & $6.7 \pm 1.4$ & 3 \\
Exe estuary & 1.0 & 2.6 & 0.6 & $3.1 \pm 0.3$ & 1 \\
Netarts bay & 0.8 & 1.2 & 0.6 & $1.9 \pm 0.2$ & 1 \\
\hline
\end{tabular}

Table 3.2: Measured wavelength $\lambda_{\text {pref }}^{M}$, mode number $n_{\text {pref }}^{M}$, width $B$, undisturbed depth $H$ and $\mathrm{M}_{2}$ current amplitude $U$. 

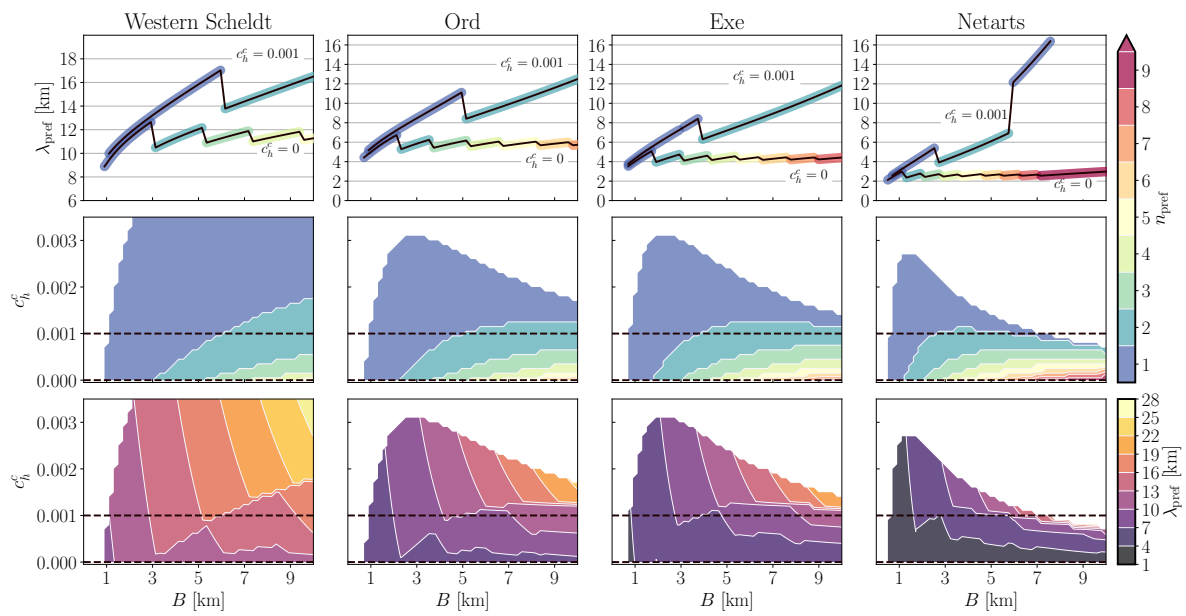

Figure 3.4: Top row: Preferred bar wavelength $\lambda_{\text {pref }}$ versus channel width $B$ for the four different channels and two values of the eddy diffusivity parameter $c_{h}^{c}$. The colors indicate the preferred mode number $n_{\text {pref }}$. Middle row: Preferred mode number $n_{\text {pref }}$ versus channel width $B$ and eddy diffusivity parameter $c_{h}^{c}$ for the four different channels. Bottom row: As middle row, but the preferred wavelength $\lambda_{\text {pref }}$ versus channel width $B$ and eddy diffusivity parameter $c_{h}^{c}$. The lines in the top row are two sections of the middle and bottom row (dashed lines). The white areas in the middle and bottom panels denote that for these parameters there does not exists a pair $(k, n)$ for which $\omega(k, n)>0$ (no bottom perturbation pattern grows). The parameters are as in Table 3.1 and 3.2 with $c_{h}^{u}=0$ and $u_{c}=0 \mathrm{~m} \mathrm{~s}^{-1}$.

of Schramkowski et al. (2002), where the preferred wavelength, as Leuven et al. (2016) noted, remains of the same order of magnitude over the whole range of channel widths. Small increases in channel width result in slight increases of the wavelength $\lambda_{\text {pref }}$. However, when the width $B$ is increased even more, a pattern with a larger mode number $n$ but with a smaller $\lambda_{\text {pref }}$ has the largest growth rate. That is, the preferred mode number jumps to a higher one.

The upper lines in the top panels of Figure 3.4 show the preferred wavelength versus channel width for the same parameter values, but with a non zero horizontal eddy diffusivity. They reveal that, for all four channels, when horizontal eddy diffusivity $\mu=c_{h}^{c} U B$, is non zero, the relative change of the preferred wavelength $\lambda_{\text {pref }}$ over the considered range of channel widths is larger than when the horizontal eddy diffusivity is neglected. That is, when $\mu \neq 0$ the preferred wavelength $\lambda_{\text {pref }}$ is more dependent on channel width $B$ than when $\mu=0$. The jumps in mode number occur later than in the case when horizontal eddy diffusivity is neglected. In fact, the middle (and top) row shows that the preferred mode number $n_{\text {pref }}$ decreases when the eddy diffusivity is taken into account. That is, the addition of horizontal eddy diffusivity reduces the growth 
of patterns with large gradients (high mode numbers and wavenumbers).

The addition of eddy viscosity or a critical velocity of erosion does not strongly alter the relation between the preferred wavelength and channel width. This is shown in Figure 3.5 for the Western Scheldt case. The right panels show the preferred wavelength versus channel width when eddy viscosity is taken into account. The left panels show the same when the critical velocity for erosion is non zero. The eddy diffusivity is neglected in the top panels while it is taken into account in the lower panels. The difference between the lines in the top panels is minimal compared to the lower line in the top left panel of Fig 3.4, where both the eddy viscosity and the critical velocity for erosion are zero.
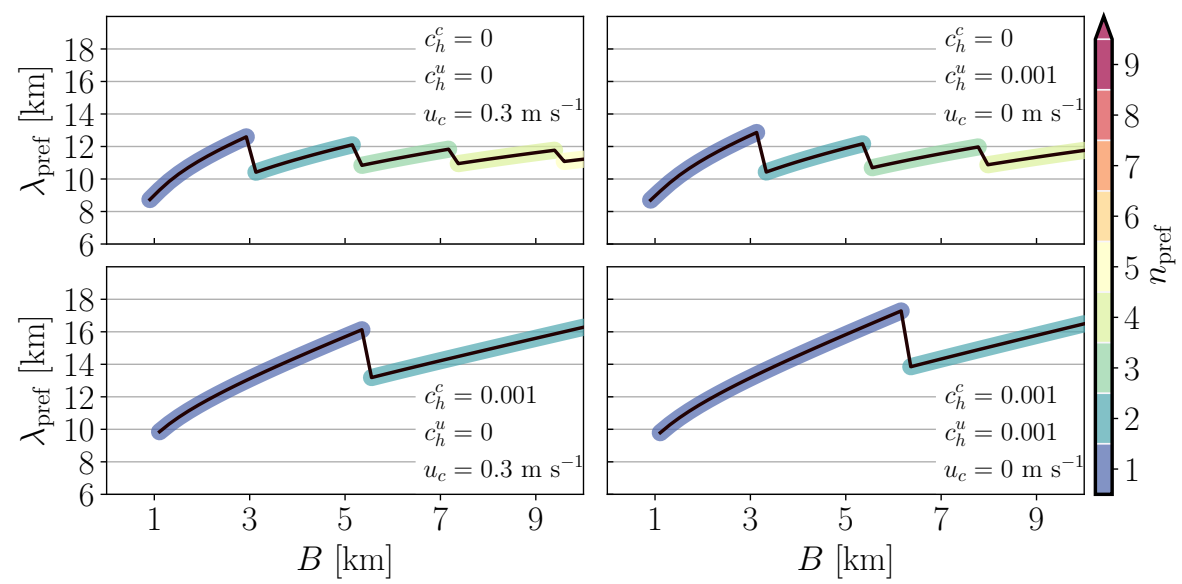

Figure 3.5: Preferred wavelength $\lambda_{\text {pref }}$ versus channel width $B$. In the left panels a critical erosion velocity is included and in the right panels eddy viscosity is included. In the top panels the eddy diffusivity is neglected while the lower panels it is not. The remaining parameters are as in Table 3.1 and 3.2 for the Western Scheldt.

\subsubsection{Different contributions to the growth rate $\omega$}

The third objective is to explain why the incorporation of the horizontal eddy diffusivity results in a dependence of the preferred wavelength $\lambda_{\text {pref }}$ on channel width $B$. To facilitate the subsequent discussion in which the third objective is met, we analyse the different terms of the growth rate $\omega$ in more depth. To simplify expressions, the critical velocity of erosion $u_{c}$ and the horizontal eddy viscosity $\nu$ are henceforth neglected, since in the previous section it is shown they do not significantly alter the relation between channel width and tidal bar wavelength. 


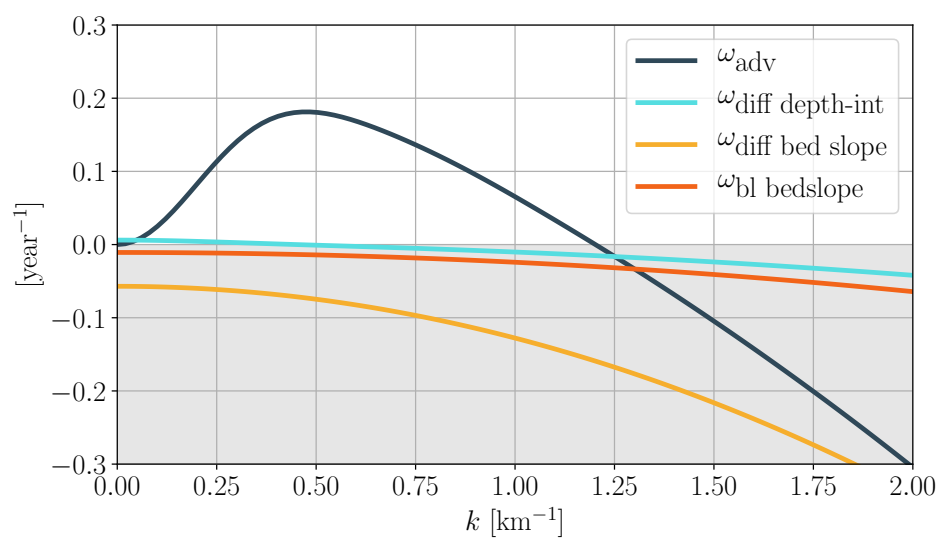

Figure 3.6: The four different parts of the growth rate $\omega$ vs wavenumber $k$ with $n=n_{\text {pref }}=2$. Parameters are as in Table 3.1 and 3.2 for the Western Scheldt case and $c_{h}^{u}=0$ and $u_{c}=0 \mathrm{~ms}^{-1}$.

The growth rate consists of four terms,

$$
\begin{aligned}
& \omega=\frac{-1}{1-\hat{p}}(\underbrace{\frac{i k U}{2}\left(\tilde{C}_{1}+\tilde{C}_{-1}\right)}_{\omega_{\mathrm{adv}}}+\underbrace{\mu\left(k^{2}+l_{n}^{2}\right) \tilde{C}_{0}}_{\omega_{\text {diff depth-int }}}+\underbrace{\mu \frac{w_{s}}{\kappa_{v}} \beta_{b} \tilde{C}_{\mathrm{eq}, 0}\left(k^{2}+l_{n}^{2}\right)}_{\omega_{\text {diff bed slope }}} \\
& +\underbrace{\Lambda\left(k^{2}+l_{n}^{2}\right)}_{\omega_{\mathrm{bl} \mathrm{bed} \mathrm{slope}}})
\end{aligned}
$$

where the factor $-1 /(1-\hat{p})$ is understood to be part of the different terms. The first term $\omega_{\mathrm{adv}}$, results from advective sediment transport. The second and third term, $\omega_{\text {diff depth-int }}$ and $\omega_{\text {diff bed slope, are due to diffusive sediment }}$ transport and the fourth term $\omega_{\mathrm{bd}}$ bedslope originates from the effect of bed slopes on bed load sediment transport. Figure 3.6 shows the contribution of the different mechanisms to the growth rate versus wavenumber $k$ for parameter values representative for the Western Scheldt. Next, we discuss the physics of the different terms of the growth rate $\omega$.

The first term in equation (3.3.1) is the advective part of the growth rate $\omega_{\text {adv }}$ and relates to convergence of tidally averaged advective sediment transport, i.e.,

$$
-\left\langle\nabla \cdot\left(\boldsymbol{u}^{\prime} C_{\mathrm{eq}}+\boldsymbol{u}_{\mathrm{eq}} C^{\prime}\right)\right\rangle
$$

To analyze $\omega_{\mathrm{adv}}$, consider the $p$-th Fourier mode of the linearized concentration 


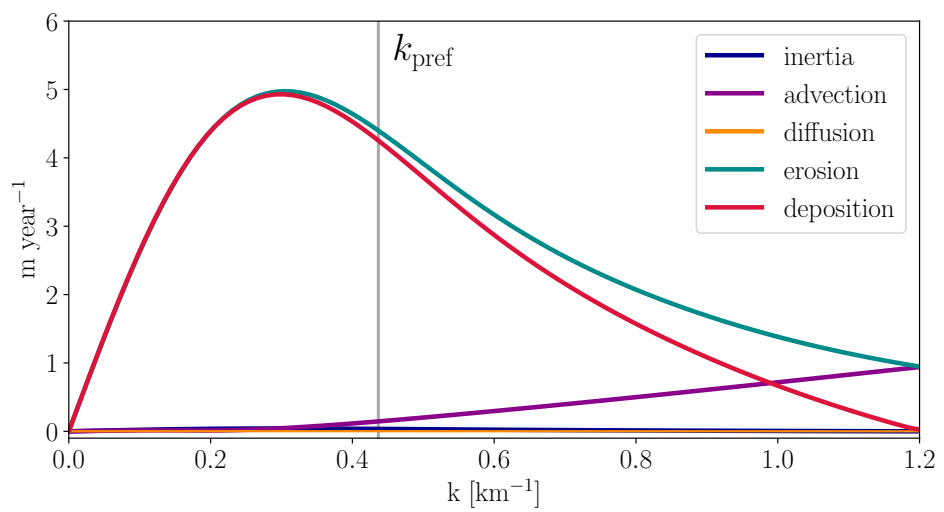

Figure 3.7: The absolute value of each term in one of the $\mathrm{M}_{2}(p=1)$ concentration equations (3.3.2). Parameters are as in Table 3.1 and 3.2 for the Western Scheldt case and $c_{h}^{u}=0, u_{c}=0 \mathrm{~ms}^{-1}$ and $n=n_{\text {pref }}=2$. The blue curve (inertia) lies below the yellow line (diffusion). When horizontal eddy diffusivity is neglected (as in Schramkowski et al., 2002), the main balance remains between erosion and deposition for $k \approx k_{\text {pref }}$, but $n_{\text {pref }}=3$ and $k_{\text {pref }}$ is larger.

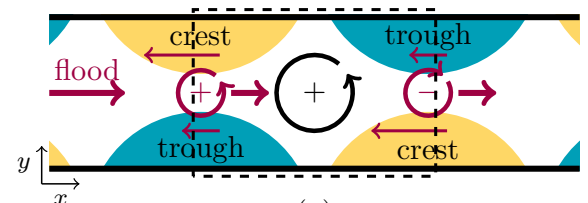

(a)

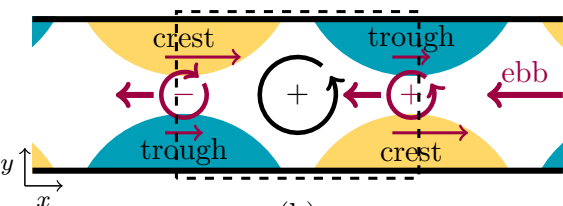

(b)

Figure 3.8: Generation of tidal residual vorticity cells for bottom pattern with mode number $n=1$. (a) Situation during flood $\left(u_{\mathrm{eq}}>0\right)$. (b) Situation during ebb $\left(u_{\mathrm{eq}}<\right.$ $0)$. Friction (thin red arrows) experienced by water column is larger on the crests than on the troughs and generates tidal vorticity (red circles). Both during ebb and flood, positive vorticity is transported into the dashed box and the negative vorticity is transported out, resulting in the build-up of positive vorticity (black circle).

equation (3.2.3) with $u_{c}=0$,

$$
\begin{aligned}
& \underbrace{i p \sigma \tilde{C}_{p}}_{\text {inertia }}+\underbrace{\frac{i k U}{2}\left(\left(\tilde{C}_{p+1}+\tilde{C}_{p-1}\right)+\tilde{C}_{\mathrm{eq}, p+1}+\tilde{C}_{\mathrm{eq}, p-1}\right)}_{\text {advection }} \\
& +\underbrace{\mu\left(k^{2}+l_{n}^{2}\right)\left(\tilde{C}_{p}+\frac{w_{s}}{\kappa_{v}} \beta_{b} \tilde{C}_{\mathrm{eq}, p}\right)}_{\text {diffusion }}=\underbrace{\alpha U\left(\tilde{u}_{p+1}+\tilde{u}_{p-1}\right)}_{\text {erosion }}-\underbrace{\gamma \tilde{C}_{p} .}_{\text {deposition }}
\end{aligned}
$$


The dominant balance in the $\mathrm{M}_{2}$ concentration equation for $k \approx k_{\text {pref }}$ is between erosion and deposition in all four considered cases. For the Western Scheldt this is shown in Figure 3.7. Then, neglecting the $\mathrm{M}_{4}$ components in the perturbed current $u^{\prime}$ (as they are small compared to the $\mathrm{M}_{2}$ components), the $\mathrm{M}_{2}$ components of the perturbed concentration reads

$$
\tilde{C}_{ \pm 1} \approx \frac{\alpha U}{\gamma} \tilde{u}_{0}
$$

Substituting the latter in $\omega_{\mathrm{adv}}$ shows that the contribution of the advective transport scales with the magnitude of the residual current $\tilde{u}_{0}$ :

$$
\omega_{\mathrm{adv}} \approx \frac{-1}{1-\hat{p}} \frac{\alpha i k U^{2}}{\gamma} \tilde{u}_{0}
$$

The physical mechanism can now be understood by analyzing the residual current $u_{0}$ via vorticity arguments that follow from Zimmerman (1981). For this, consider a longitudinal tidal current that moves over a bottom that consists of bars and troughs as illustrated in Figure 3.8 (here the $n=1$ pattern is assumed). The lateral depth variations result in frictional torques that generate tidal vorticity, as is indicated by the red circles. This vorticity is subsequently transported by the unperturbed tidal flow. As a result, both during ebb and flood there is an influx of vorticity of the same sign into a region between crests and troughs. Thus, residual vorticity builds up in these areas (black circles in the figure), which is balanced by dissipation due to bottom friction. The resulting residual currents turn out to be directed from the troughs to the crests in the longitudinal direction and from the crest to the troughs in the lateral direction (see also right column in Figure 3.3). To understand why this leads to growth of bars and deepening of troughs, consider the convergence of the tidally averaged advective sediment transport (see equation (3.2.8) and (3.2.9)). This reads, using that the equilibrium velocity $u_{\mathrm{eq}}$ and the equilibrium sediment concentration $C_{\text {eq }}$ are spatially uniform,

$$
-\left\langle C_{\mathrm{eq}} \boldsymbol{\nabla} \cdot \boldsymbol{u}^{\prime}\right\rangle-\left\langle\boldsymbol{u}_{\mathrm{eq}} \cdot \boldsymbol{\nabla} C^{\prime}\right\rangle=-\left\langle C_{\mathrm{eq}} u_{\mathrm{eq}}\right\rangle \frac{1}{H} \frac{\partial h^{\prime}}{\partial x}-\left\langle u_{\mathrm{eq}} \frac{\partial C^{\prime}}{\partial x}\right\rangle=-\left\langle u_{\mathrm{eq}} \frac{\partial C^{\prime}}{\partial x}\right\rangle,
$$

where for the first equality sign, the perturbed continuity equation is used and for the second one the fact that $\left\langle u_{\text {eq }} C_{\text {eq }}\right\rangle=0$. Equation (3.3.5) shows that convergence of sediments occurs when $u_{\text {eq }}$ and $\partial C^{\prime} / \partial x$ are negatively correlated. From equation (3.3.3) it follows that the perturbed concentration reads

$$
C^{\prime} \approx \frac{2 \alpha}{\gamma} u_{0} u_{\mathrm{eq}}
$$

Since $u_{0}$ is directed towards the bars, this implies that both during ebb and flood the perturbed concentration $C^{\prime}$ is positive upstream from a bar and negative downstream. On the other hand, $C^{\prime}$ is negative upstream a trough and 


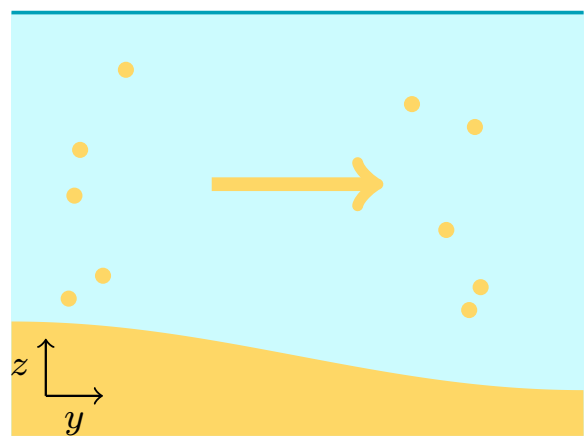

Figure 3.9: Consider a uniform depth-integrated concentration over a sloping bottom. On the left, the water column is shallower than on the right. Hence, the concentration $C_{3}(t, x, y, z)$ is larger on the left than on the right. As a result, sediments diffuse from left to right even though the depth-integrated concentrations are equal. In this case, it is the gradient in bed level that induces diffusive sediment transport instead of a gradient in depth-integrated sediment concentration.

positive downstream of it. Hence, the convergence of advective transport causes bars to grow and troughs to deepen for patterns with wavenumber $k$ and mode number $n$ close to the preferred ones. Figure 3.6 reveals that for larger $k, \omega_{\text {adv }}$ is negative. The reason is that for these patterns the dominant balance in the concentration equation is not between erosion and deposition but that the advection term also becomes significant (Figure 3.7).

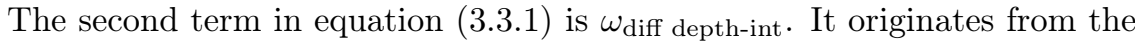
fact that when at one location the depth-integrated concentration $C$ is larger than at another, a transport occurs from high to low $C$. Figure 3.6 reveals that $\omega_{\text {diff depth-int }}$ is slightly positive for small $k$ and negative for larger $k$ and close to zero for wavenumbers close to the preferred wavenumber $k_{\text {pref }}$. That is, for patterns with large wavenumbers, the depth-integrated concentration is larger above the troughs than above the crests, while the opposite is the case for patterns with small wavenumbers. Therefore, the depth-integrated diffusive transport results in a small contribution of the growth of patterns with large wavenumbers and slightly causes decay of patterns with small wavenumbers.

The third term in equation (3.3.1) $\omega_{\text {diff bed slope, }}$ relates to a suspended load bed slope effect. It arises in the derivation of the depth-integrated concentration equation (3.2.3) from the $3 \mathrm{D}$ concentration equation when the diffusion term is integrated over depth,

$$
\int_{h(\boldsymbol{x})}^{H} \nabla \cdot\left(\mu \nabla C_{3}\right) \mathrm{d} z=\nabla \cdot(\mu \nabla C)+\nabla \cdot\left(\mu c_{b} \nabla h\right),
$$

where $C_{3}$ is the ' $3 \mathrm{D}$ concentration' depending on $x, y$ and $z$, and $c_{b}$ the concentration at the reference level above the bottom $z=h(\boldsymbol{x})$. When a balance 
is assumed between vertical turbulent mixing (with vertical eddy diffusivity $\kappa_{v}$ constant over the vertical) and downward settling, the concentration at the bottom reads $c_{b}=\left(w_{s} / \kappa_{v}\right) \beta_{b} C$ (ter Brake and Schuttelaars, 2010). The first term in equation (3.3.6) results in $\omega_{\text {diff depth-int }}$ and the second in $\omega_{\text {diff bed slope }}$ The physics behind the second term becomes apparent when considering for example, a sloping bed and a uniform depth-integrated concentration. Then, the first term $\boldsymbol{\nabla} \cdot(\mu \boldsymbol{\nabla} C)$, is zero. However, in the shallow region the concentration $C_{3}$ is higher than in the deep region, leading to sediment transport from the shallow to the deep region (Figure 3.9). The second term models the convergence of this transport. As the magnitude of sediment transport from crests to troughs scales with the slope, $\omega_{\text {diff bed slope }}$ is negative and becomes increasingly more negative if bottom gradients increase.

The last term of equation (3.3.1), $\omega_{\mathrm{bl}}$ bed slope , relates to bottom slope effects on bed load transport: sediment moves easier downslope than upslope. Therefore contributes $\omega_{\mathrm{bl}}$ bed slope, like the third term, to convergence of sediments in the troughs and divergence of sediments on the crests. Hence, it dampens the growth of the pattern. That is, $\omega_{\mathrm{bl}}$ bed slope $<0$ and is stronger negative

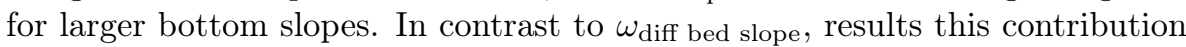
from bed load transport processes. Figure 3.6 reveals that $\omega_{\text {bl bed slope }}$ is much smaller than $\omega_{\text {diff bed slope, }}$ which, based on the values from Table 3.1, directly follows from the fact that

$$
\frac{\mu w_{s} \beta_{b} \tilde{C}_{\mathrm{eq}, 0} / \kappa_{v}}{\Lambda} \approx 5
$$

The above gives a physical argument for using a larger bed load bottom slope parameter $\Lambda$ than suggested by literature (Baar et al., 2018), when a total load formula is used. It then also represents the suspended load bed slope effect when this is not explicitly taken into account.

\subsection{Discussion}

\subsubsection{Mechanism behind sensitivity of tidal bar wave- length to channel width}

Here, we address the third objective by discussing three important aspects of Figure 3.4, which shows tidal bar wavelength $\lambda_{\text {pref }}$ versus channel width $B$.

A1. In the range of channel widths with constant $n_{\text {pref }}, \lambda_{\text {pref }}$ increases with increasing $B$.

A2. While increasing $B$, the preferred wavelength $\lambda_{\text {pref }}$ decreases when $n_{\text {pref }}$ jumps to a higher mode number. 
A3. The range of channel widths for which $n_{\text {pref }}$ is the same is wider when horizontal eddy diffusivity $\mu$ in the sediment mass balance is taken into account.

Together, this shows that the wavelength depends on channel width when horizontal eddy diffusivity is taken into account and why the wavelength remains of the same order of magnitude for a large range of channel widths when the horizontal eddy diffusivity is neglected.

To analyse the three aspects above, the growth rate is first approximated as

$$
\tilde{\omega} \approx \omega_{\text {adv }}+\omega_{\text {eff bed slope }}
$$

Here, the two bed slope effects $\omega_{\text {diff bed slope }}$ and $\omega_{\mathrm{bl}}$ bed slope are combined into an effective bed slope term

$$
\omega_{\text {eff bed slope }}=\frac{-1}{1-\hat{p}} \Lambda_{\text {eff }}\left(k^{2}+l_{n}^{2}\right), \quad \text { with } \quad \Lambda_{\text {eff }}=\Lambda+\mu \frac{w_{s}}{\kappa_{v}} \beta_{b} \tilde{C}_{\text {eq }, 0},
$$

and $\omega_{\text {diff depth-int }}$ is neglected.

The first two aspects (A1 and A2) follow from the fact that, for a fixed mode number, the growth rate is sensitive to changes in channel width. For a fixed mode number $n$, the wavelength $\tilde{\lambda}$ for which $\tilde{\omega}$ is maximal is approximately (see Appendix 3.6.3)

$$
\tilde{\lambda}(n)=2^{3 / 4}\left(\frac{\pi B}{n}\right)^{1 / 2}\left(\mathcal{L}_{\mathrm{f}}^{-2}+\mathcal{L}_{\mathrm{t}}^{-2}\right)^{-1 / 4},
$$

where

$$
\mathcal{L}_{\mathrm{f}}=\frac{H}{8 /(3 \pi) c_{d}} \quad \text { and } \quad \mathcal{L}_{\mathrm{t}}=\frac{U}{\sigma} .
$$

Here, $\mathcal{L}_{\mathrm{f}}$ is a friction length scale and $\mathcal{L}_{\mathrm{t}}$ the tidal excursion length. When $n=$ $n_{\text {pref }}$ in equation $(3.4 .3), \tilde{\lambda}\left(n_{\text {pref }}\right)$ is a first order approximation for the preferred wavelength $\lambda_{\text {pref }}$ (A higher order approximation is given in Appendix 3.6.3). Equation (3.4.3) therefore yields an approximate relation between the preferred wavelength and three internal length scales of the system: the channel width $B$, the friction length scale $\mathcal{L}_{\mathrm{f}}$ and the tidal excursion length $\mathcal{L}_{\mathrm{t}}$. In its derivation the approximate balance between erosion and deposition, see equation (3.3.3), is used and $\nu$ and $u_{c}$ are assumed to be zero, since including horizontal eddy viscosity $\nu$ in the momentum balance or critical erosion velocity $u_{c}$ was shown to be important for the dependence on channel width. The first aspect (A1) is revealed by equation (3.4.3); in wider channels with the same mode number, $\tilde{\omega}$ is maximal for larger wavelengths $\tilde{\lambda}$. The second aspect (A2) also becomes apparent from equation (3.4.3). It shows that the preferred wavelength of patterns with higher mode number is smaller and hence $\lambda_{\text {pref }}$ decreases when the preferred mode number $n_{\text {pref }}$ jumps to a higher mode number. 
The third aspect (A3) boils down to the statement that for a fixed channel width $B$, the preferred mode number $n_{\text {pref }}$ is lower when $\mu \neq 0$ than when $\mu=0$. This follows from a competition between the two mechanisms related to the first and second term in equation (3.4.1). The first term increases while the second term decreases with increasing mode number $n$. That is, the advective term 'promotes' strong lateral gradients in the bed, while the bed slope term does the opposite. As a result, if $\Lambda_{\text {eff }}$ increases, $n_{\text {pref }}$ decreases. In fact, as shown in Schramkowski et al. (2002), if $\Lambda_{\text {eff }}=0$, the preferred mode number $n_{\text {pref }}$ tends to infinity, but when $\Lambda_{\text {eff }} \neq 0$, the preferred mode number is finite. The essential difference between the situation where $\mu \neq 0$ compared to the case where $\mu=0$ is that $\Lambda_{\text {eff }}$ is larger in the former case and thereby a lower preferred mode $n_{\text {pref }}$ is selected. Hence, the range of channel widths for which $n_{\text {pref }}$ is the same is wider when horizontal eddy diffusivity $\mu$ in the sediment mass balance is taken into account.

Van der Wegen and Roelvink (2008) found a wavelength of bar patterns that, besides its dependence on current velocity and depth, scales with the square root of the channel width. In their model, the sediment transport is governed by a total load formulation (after Engelund and Hansen, 1967), where the total transport is adjusted for bed slope effects. They state that the bed slope effect is overestimated in order to obtain realistic bottom patterns. Hence, their findings correspond to the ones above, where the dependence of the wavelength to channel width is only found if the bed slope effects are relatively strong. Furthermore, equation (3.4.3) suggests that the preferred wavelength scales with the square root of channel width. Hibma et al. (2004) used a concentration equation with the bed slope effect induced by the horizontal eddy diffusivity present in their formulation. They also found tidal bar wavelength to increase with channel width.

\subsubsection{Sensitivity of fluvial bar wavelength to river width}

Leuven et al. (2016) analysed the fluvial bar model of Crosato and Mosselman (2009), based on Struiksma et al. (1985). They showed that, like in the tidal bar case, the number of fluvial bars and channels in the lateral direction of the channel increases with increasing river width. Furthermore, the model of Crosato and Mosselman (2009) reveals that, for a constant mode number, the increase of bar wavelength with increasing river width and also the drop in bar wavelength when the mode number increases. Moreover, when their bed slope parameter is increased, the mode number decreases. This suggests that in rivers where suspended sediment transport is significant, the horizontal eddy diffusivity will increase the sensitivity of fluvial bar wavelength to river width by the same mechanism as described above. Note there are differences: fluvial bars migrate, whilst tidal bars can be steady (as is the case in this study with a symmetric equilibrium current). 

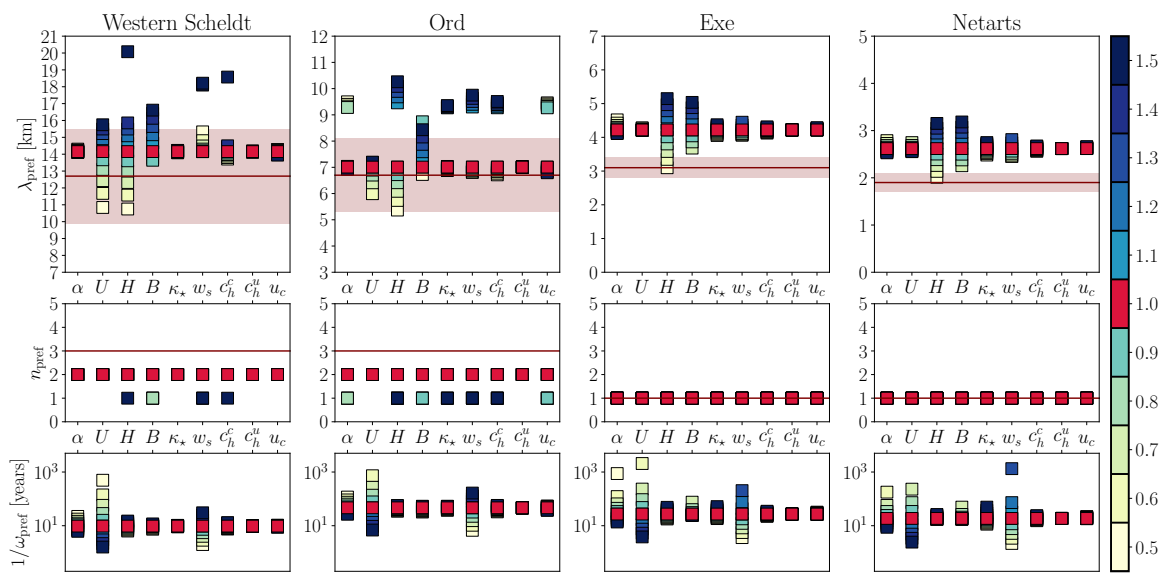

Figure 3.10: The preferred wavelength $\lambda_{\text {pref }}$, mode number $n_{\text {pref }}$ and $e$-folding growth time $1 / \omega_{\text {pref }}$ versus different parameter values for modelled tidal bars in four different tidal channels. The colors indicate the factor with which the default parameter value is multiplied, with the red squares representing the default setting for that channel. The red lines denote the measured wavelength $\lambda_{\text {pref }}^{M}$ and mode number $n_{\text {pref }}^{M}$ as in Table 3.2 and the red area the measurement uncertainty (as in Figure 3.3). Parameter $\alpha$ affects the erosion, $U$ the amplitude of the basic state tidal velocity, $H$ the undisturbed depth, $B$ the channel width, $\kappa_{\star}$ the bed load bed slope term, $w_{s}$ the settling velocity, $c_{h}^{c}$ the horizontal eddy diffusivity, $c_{h}^{u}$ the horizontal eddy viscosity and $u_{c}$ the critical erosion. The parameters values are as in Table 3.1 and 3.2 with $c_{h}^{u}=c_{h}^{c}=0.001$ and $u_{c}=0.3 \mathrm{~m} \mathrm{~s}^{-1}$.

\subsubsection{Sensitivity bar wavelength to other parameters}

In concurrence with findings of Hibma et al. (2004), Leuven et al. (2016) and van der Wegen and Roelvink (2008), it follows from equation (3.4.3) that the preferred wavelength is, besides the channel width, sensitive to changes in current velocity and channel depth. It is therefore relevant to assess the sensitivity of the modelled $\lambda_{\text {pref }}$ to these other parameters. Figure 3.10 shows the preferred wavelength $\lambda_{\text {pref }}$, the preferred mode number $n_{\text {pref }}$ and the $e$-folding time scale $1 / \omega_{\text {pref }}$ for the four tidal channels that were introduced in Section 3.3 (see also Figure 3.1). The red squares denote the results using the default parameter values for that channel (corresponding to Figure 3.3). In Figure 3.10, each default value is multiplied by factors between 0.5 and 1.5 to illustrate the sensitivity of $\lambda_{\text {pref }}, n_{\text {pref }}$ and $1 / \omega_{\text {pref }}$ to these parameters. The figure reveals that, in particular for the Western Scheldt and Ord river estuary, $\lambda_{\text {pref }}$ is sensitive to both current velocity $U$, depth $H$ and channel width $B$. In the Exe estuary and 
Netarts bay, the current has less influence on the preferred wavelength than in the other two channels. The jumps in $\lambda_{\text {pref }}$ in the top panels correspond to jumps in mode number $n_{\text {pref }}$. The growth rate is most sensitive to current velocity and settling velocity.

To further analyse the sensitivity of the preferred wavelength to the current velocity and the depth, differentiate the wavelength $\tilde{\lambda}$ with respect to the length scales $B, \mathcal{L}_{\mathrm{t}}$ and $\mathcal{L}_{\mathrm{f}}$ to obtain, respectively,

$$
\begin{aligned}
\frac{\partial \tilde{\lambda}}{\partial B} & =2^{-1 / 4} \sqrt{\frac{\pi}{n B}}\left(\mathcal{L}_{\mathrm{t}}^{-2}+\mathcal{L}_{\mathrm{f}}^{-2}\right)^{-1 / 4}, \\
\frac{\partial \tilde{\lambda}}{\partial \mathcal{L}_{\mathrm{t}}} & =2^{-1 / 4} \sqrt{\frac{\pi B}{n}} \frac{1}{\mathcal{L}_{\mathrm{t}}^{3}}\left(\mathcal{L}_{\mathrm{t}}^{-2}+\mathcal{L}_{\mathrm{f}}^{-2}\right)^{-5 / 4}, \\
\frac{\partial \tilde{\lambda}}{\partial \mathcal{L}_{\mathrm{f}}} & =2^{-1 / 4} \sqrt{\frac{\pi B}{n}} \frac{1}{\mathcal{L}_{\mathrm{f}}^{3}}\left(\mathcal{L}_{\mathrm{t}}^{-2}+\mathcal{L}_{\mathrm{f}}^{-2}\right)^{-5 / 4} .
\end{aligned}
$$

The first expression approximates the slopes of the lines in the upper panels of Figure 3.4 for a fixed $n_{\text {pref }}$. It shows that when one of the length scales $B$, $\mathcal{L}_{\mathrm{t}}$ and $\mathcal{L}_{\mathrm{f}}$ is very small (large), the sensitivity of $\tilde{\lambda}$ to that length scale is large (small) compared to the others. The bottom lines in the top row of Figure 3.4 also show this behavior; the slope of the lines decreases with increasing width $B$. This was also observed by Leuven et al. (2016), who found that for estuaries where the current velocity is large, the bar length hardly depends on the current velocity. Also, it agrees with the results of Dalrymple and Rhodes (1995), who found that the wavelength correlates strongly with channel width when considering channels of only a few hundred meters wide.

\subsubsection{Formulation of horizontal eddy diffusivity}

The horizontal eddy diffusivity $\mu$ is chosen to scale with the product of a velocity scale (equilibrium current velocity $U$ ) and a length scale (channel width $B$ ) such that its order of magnitude is $10 \mathrm{~m}^{2} \mathrm{~s}^{-1}$ (Deltares, 2019). One observation from Figure 3.4 was that the relative change of the tidal bar wavelength over the considered range of channel widths is larger when horizontal eddy diffusivity $\mu$ was taken into account. Figure 3.11 reveals that is also the case when the parameterization of $\mu$ is independent of channel width $B$.

\subsection{Conclusions}

The model of Schramkowski et al. (2002) for tidal bars in channels is extended to include horizontal eddy diffusivity, horizontal eddy viscosity and a critical velocity for erosion. It is shown that this model is able to mimic tidal bar patterns with length scales and growth times that are of the same order 


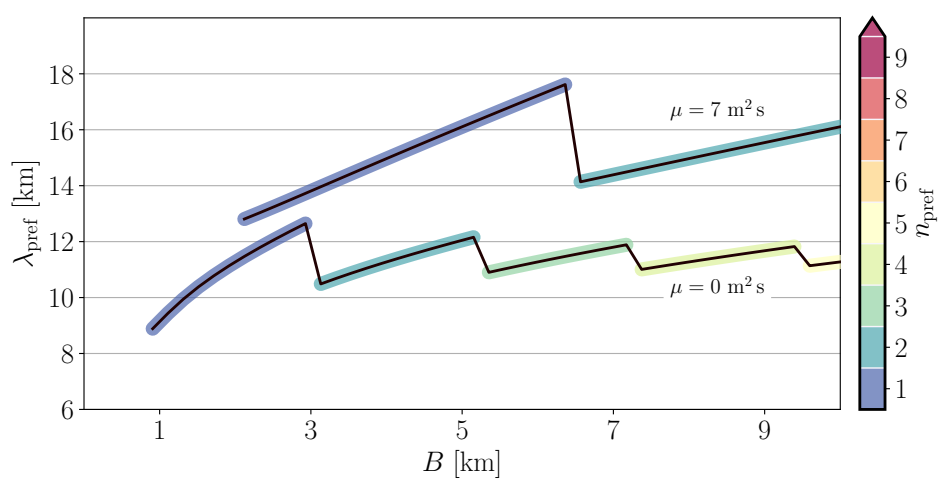

Figure 3.11: Preferred bar wavelength $\lambda_{\text {pref }}$ versus channel width $B$ for two values of eddy diffusivity $\mu$ (independent of channel width $B$ ). The colors denote the preferred mode number $n_{\text {pref }}$. Parameters are as in Table 3.1 and 3.2 for the Western Scheldt case and $c_{h}^{u}=0$ and $u_{c}=0 \mathrm{~ms}^{-1}$.

of magnitude as those of observed bars, including the sensitivity of tidal bar wavelength to channel width. In particular, the observed sensitivity of tidal bar wavelength to channel width was reproduced by taking the eddy diffusivity into account. The inclusion of horizontal eddy viscosity and critical erosion velocity did not significantly alter the relation between the tidal bar wavelength and the channel width.

The reason the extended model shows a clear dependence of tidal bar wavelength on channel width, whilst in the original model of Schramkowski et al. (2002) this dependence was weak, is the following. First of all, in wider channels, the pattern with the largest growth rate (the preferred tidal bar pattern) has more bars in the lateral direction. That is, it has a higher preferred mode number. Second, in the range of channel widths with constant preferred mode number, the preferred wavelength increases with increasing channel width. However, at the width where the preferred mode number changes (it increases by one), the preferred wavelength decreases. In the model of Schramkowski et al. (2002), the range of channel widths with constant preferred mode number is relatively short. This means that, with increasing channel width, before the preferred wavelength can increase significantly, the preferred mode number increases and with that, the wavelength decreases again. As a result, the tidal bar wavelength remains of the same order of magnitude for different values of channel width. In the present study, the effects of horizontal eddy diffusivity are added to the model. As a result, the effective bed slope effect is stronger. Since bed slope effects decrease the growth rate of bottom patterns with large gradients, the bottom pattern with the largest growth rate has a smaller mode number. This implies that the preferred mode number remains the same for a wider range of channel widths than in the model of Schramkowski et al. (2002). 
The width dependence now follows from the fact that, in this range, the tidal bar wavelength increases with increasing channel width and that this increase is stronger for smaller mode numbers.

\subsection{Appendix}

\subsubsection{Equilibrium pressure gradient and concentration}

The equilibrium pressure gradient and sediment concentration are

$$
\left(\boldsymbol{F}_{\boldsymbol{p}}\right)_{\mathrm{eq}}=\left(\frac{\partial u_{\mathrm{eq}}}{\partial t}+\frac{r u_{\mathrm{eq}}}{H-h}, 0\right) \text { and } C_{\mathrm{eq}}=\sum_{p=-\infty}^{\infty} \tilde{C}_{e q, p} e^{i p \sigma t},
$$

with

$$
\tilde{C}_{e q, p}=\frac{\alpha}{i p \sigma+\gamma}\left[\frac{U^{2}}{4}\left(\tilde{\mathcal{H}}_{p-2}+2 \tilde{\mathcal{H}}_{p}+\tilde{\mathcal{H}}_{p+2}\right)-\tilde{\mathcal{H}}_{p} u_{c}^{2}\right] .
$$

In this expression

$$
\mathcal{H}\left(\left\|\boldsymbol{u}_{\text {eq }}\right\|^{2}-u_{c}^{2}\right)=\sum_{p=-\infty}^{\infty} \tilde{\mathcal{H}}_{p} e^{i p \sigma t}
$$

with

$$
\tilde{\mathcal{H}}_{p}= \begin{cases}\frac{i}{2 \pi p}\left(\left[e^{-i p \sigma t}\right]_{-\pi / \sigma}^{t_{3}}+\left[e^{-i p \sigma t}\right]_{t_{2}}^{t_{1}}+\left[e^{-i p \sigma t}\right]_{t_{4} / \sigma}^{\pi / \sigma}\right) & p \neq 0 \\ \frac{\sigma}{2 \pi}\left(t_{3}+\frac{\pi}{\sigma}+t_{1}-t_{2}+\frac{\pi}{\sigma}-t_{4}\right) & p=0\end{cases}
$$

and

$$
t_{1}=\frac{1}{2 \sigma} \cos ^{-1}\left(2\left(\frac{u_{c}}{U}\right)^{2}-1\right), \quad t_{2}=-t_{1}, \quad t_{3}=t_{1}-\frac{\pi}{\sigma}, \quad t_{4}=-t_{3} .
$$

\subsubsection{Linearized equations}

The system of partial differential equations in the perturbed variables reads

$$
\begin{array}{ll}
H\left(\boldsymbol{\nabla} \cdot \boldsymbol{u}^{\prime}\right)-\boldsymbol{u}_{\mathrm{eq}} \cdot \boldsymbol{\nabla} h^{\prime} & =0 \\
\frac{\partial \boldsymbol{u}^{\prime}}{\partial t}+\left(\boldsymbol{u}_{\mathrm{eq}} \cdot \boldsymbol{\nabla}\right) \boldsymbol{u}^{\prime}-\nu \nabla^{2} \boldsymbol{u}^{\prime}+\boldsymbol{F}_{\boldsymbol{r}}^{\prime}+g \boldsymbol{\nabla} \zeta^{\prime} & =0 \\
\frac{\partial C^{\prime}}{\partial t}+C_{\mathrm{eq}}\left(\boldsymbol{\nabla} \cdot \boldsymbol{u}^{\prime}\right)+\boldsymbol{u}_{\mathrm{eq}} \cdot \nabla C^{\prime}-\mu\left(\nabla^{2} C^{\prime}+\frac{w_{s}}{\kappa_{v}} \beta_{b} C_{\mathrm{eq}} \nabla^{2} h^{\prime}\right) & \\
-\left(E^{\prime}-D^{\prime}\right) & =0 \\
(1-\hat{p}) \frac{\partial h^{\prime}}{\partial t}+\boldsymbol{\nabla} \cdot\left\langle\boldsymbol{q}_{\mathrm{s}}^{\prime}+\boldsymbol{q}_{\mathrm{b}}^{\prime}\right\rangle & =0
\end{array}
$$


with

$$
\boldsymbol{F}_{\boldsymbol{r}}^{\prime}=\left(\frac{r u_{\mathrm{eq}} h^{\prime}}{H^{2}}+\frac{r u^{\prime}}{H}, \frac{r v^{\prime}}{H}\right), \quad E^{\prime}=2 \alpha u_{\mathrm{eq}} u^{\prime} \mathcal{H}\left(\left\|\boldsymbol{u}_{\mathrm{eq}}\right\|^{2}-u_{c}\right), \quad D^{\prime}=\gamma C^{\prime},
$$

and

$$
\boldsymbol{q}_{\mathrm{s}}^{\prime}=\boldsymbol{u}^{\prime} C_{\mathrm{eq}}+\boldsymbol{u}_{\mathrm{eq}} C^{\prime}-\mu\left(\nabla C^{\prime}+\frac{w_{s}}{\kappa_{v}} \beta_{b} C_{\mathrm{eq}} \nabla h^{\prime}\right) \quad \text { and } \quad \boldsymbol{q}_{\mathrm{b}}^{\prime}=-\Lambda \nabla h^{\prime} .
$$

The boundary conditions are

$$
v^{\prime}=0, \quad \frac{\partial C^{\prime}}{\partial y}=0, \quad \frac{\partial h^{\prime}}{\partial y}=0 \quad \text { and } \quad \frac{\partial u^{\prime}}{\partial y}=0 \quad \text { at } y=0, B .
$$

As variables $\boldsymbol{u}, \boldsymbol{F}_{p}$ and $C$ are assumed bounded, this must also hold for their primed parts.

\subsubsection{Approximation of preferred wavelength}

In this appendix, we approximate the wavelength for which

$$
\tilde{\omega}=\omega_{\mathrm{adv}}+\omega_{\text {bed slope }}
$$

is maximal for a fixed mode number $n$. For this, an approximate expression is derived for

$$
\omega_{\mathrm{adv}}=\frac{-1}{1-\hat{p}} \frac{i k U}{2}\left(\tilde{C}_{1}+\tilde{C}_{-1}\right) .
$$

Assume that $\mu=0$ and $u_{\mathrm{eq}}=U \cos (\sigma t)$. In the main text it is shown that around $k_{\text {pref }}$ the main balance in the concentration equation is between erosion and deposition so that, with equation (3.3.3),

$$
\omega_{\mathrm{adv}} \approx \frac{-1}{1-\hat{p}} \frac{\alpha i k U^{2}}{\gamma} \tilde{u}_{0}
$$

Next, we will approximate $\tilde{u}_{0}$ via the residual vorticity. Denote the perturbed vorticity by

$$
\Omega^{\prime}=\frac{\partial v^{\prime}}{\partial x}-\frac{\partial u^{\prime}}{\partial y}=\operatorname{Re}\left\{\hat{h} \sum_{p=-\infty}^{\infty} \tilde{\Omega}_{p} \sin \left(l_{n} y\right) e^{i(k x+p \sigma t)}\right\}
$$

where $\tilde{\Omega}_{p}=i k \tilde{v}_{p}+l_{n} \tilde{u}_{p}$. From the tidally averaged continuity equation $\nabla$. $\left\langle\boldsymbol{u}^{\prime}\right\rangle=0$, it follows that the residual velocity $\tilde{u}_{0}$ reads, in terms of the residual vorticity $\tilde{\Omega}_{0}$,

$$
\tilde{u}_{0}=\frac{l_{n}}{k^{2}+l_{n}^{2}} \tilde{\Omega}_{0} .
$$


To approximate the residual vorticity $\tilde{\Omega}_{0}$ consider the vorticity equation,

$$
\frac{\partial \Omega^{\prime}}{\partial t}+u_{\mathrm{eq}} \frac{\partial \Omega^{\prime}}{\partial x}+\frac{r}{H} \Omega^{\prime}=\frac{r u_{\mathrm{eq}}}{H^{2}} \frac{\partial h^{\prime}}{\partial y} .
$$

It shows that vorticity is advected by the current $u_{\text {eq }}$, dissipated by friction and generated by the flow which experiences friction and lateral depth variations. For every integer $p$, the Fourier coefficients $\tilde{\Omega}_{p}$ then satisfy

$$
i p \sigma \tilde{\Omega}_{p}+\frac{i k U}{2}\left(\tilde{\Omega}_{p+1}+\tilde{\Omega}_{p-1}\right)+\frac{r}{H} \tilde{\Omega}_{p}=-\frac{r l_{n}}{H^{2}}\left(\tilde{u}_{\mathrm{eq}}\right)_{p},
$$

where $\left(\tilde{u}_{\mathrm{eq}}\right)_{p}$ denotes the $p$-th Fourier component of $u_{\mathrm{eq}}$. Since $\left(\tilde{u}_{\mathrm{eq}}\right)_{p} \neq 0$ only for $p= \pm 1$, the lateral depth gradients only generate vorticity at the $M_{2}$ frequency. However, via the advective terms, vorticity is transported from the $\mathrm{M}_{2}$ components to the residual component (as described in Figure 3.8). Neglecting the $\mathrm{M}_{4}$ components $\Omega_{ \pm 2}$, for $p=0$ and $p= \pm 1$, equation (3.6.8) yields

$$
\tilde{\Omega}_{0}=-\frac{i k U H}{2 r}\left(\tilde{\Omega}_{-1}+\tilde{\Omega}_{1}\right) \quad \text { and } \quad \tilde{\Omega}_{ \pm 1} \approx \frac{-U}{2\left(\frac{r}{H} \pm i \sigma\right)}\left(\frac{r l_{n}}{H^{2}}+i k \tilde{\Omega}_{0}\right) .
$$

Plugging the latter in the former and solving for $\tilde{\Omega}_{0}$ results in

$$
\tilde{\Omega}_{0} \approx \frac{r l_{n} i k}{H^{2}\left(k^{2}+\tau^{2}\right)} .
$$

where $\tau^{2}=2\left(\mathcal{L}_{\mathrm{f}}^{-2}+\mathcal{L}_{\mathrm{t}}^{-2}\right)$ with $\mathcal{L}_{\mathrm{f}}=H /\left(8 /(3 \pi) c_{d}\right)$ and $\mathcal{L}_{\mathrm{t}}=U / \sigma$. Combining this with equation $(3.6 .7)$ results in

$$
\tilde{u}_{0} \approx \frac{r l_{n}^{2} i k}{H^{2}\left(k^{2}+l_{n}^{2}\right)\left(k^{2}+\tau^{2}\right)} .
$$

Hence,

$$
\omega_{\mathrm{adv}} \approx \frac{-1}{1-\hat{p}} \frac{\alpha i k U^{2}}{\gamma} \tilde{u}_{0} \approx \frac{\alpha U^{2} k^{2} l_{n}^{2} r}{(1-\hat{p}) \gamma H^{2}\left(k^{2}+l_{n}^{2}\right)\left(k^{2}+\tau^{2}\right)} .
$$

Plugging this in equation (3.6.6) together with the expression for $\omega_{\text {bed slope }}$ in equation (3.4.2) yields

$$
\tilde{\omega} \approx a \frac{k^{2} l_{n}^{2}}{\left(k^{2}+l_{n}^{2}\right)\left(k^{2}+\tau^{2}\right)}-\Lambda_{\mathrm{eff}}\left(k^{2}+l_{n}^{2}\right),
$$

where

$$
a=\frac{\alpha U^{2} r}{(1-\hat{p}) \gamma H^{2}} \quad \text { and } \quad \Lambda_{\mathrm{eff}}=\Lambda+\mu \frac{w_{s}}{\kappa_{v}} \beta_{b} \tilde{C}_{\mathrm{eq}, 0}
$$


To asymptotically approximate the wavenumber for which $\tilde{\omega}$ is maximal, scale $k, l_{n}$ and $\tau$ with the tidal excursion length $\mathcal{L}_{\mathrm{t}}$,

$$
k^{*}=k \mathcal{L}_{\mathrm{t}}, \quad l_{n}^{*}=l_{n} \mathcal{L}_{\mathrm{t}} \quad \text { and } \quad \tau^{*}=\tau \mathcal{L}_{\mathrm{t}} .
$$

Substituting these in equation (3.6.11) and divide by $a$ results in

$$
\omega^{*} \approx \frac{k^{* 2} l_{n}^{* 2}}{\left(k^{*^{2}}+l_{n}^{* 2}\right)\left(k^{*^{2}}+\tau^{* 2}\right)}-\varepsilon\left(k^{* 2}+l_{n}^{* 2}\right),
$$

where $\omega^{*}=\tilde{\omega} / a$ and $\varepsilon=\Lambda_{\text {eff }} /\left(a \mathcal{L}_{t}^{2}\right)$, which is much smaller than one. To find the $k^{*}$ for which $\omega^{*}$ is maximal, differentiate $\omega^{*}$ with respect to $k^{*}$ and equate with zero to obtain

$$
l_{n}^{* 2}\left(l_{n}^{* 2} \tau^{* 2}-k^{* 4}\right)-\varepsilon\left(k^{* 2}+l_{n}^{* 2}\right)^{2}\left(k^{* 2}+\tau^{* 2}\right)^{2}=0 .
$$

Here, we divided by $2 k^{*}$, thereby excluding the pattern that is uniform in the longitudinal direction $\left(k^{*}=0\right)$, since $\omega^{*}$ obtains a local minimum there. Plugging the asymptotic expansion $k^{*}=k_{0}^{*}+\varepsilon k_{1}^{*}+O\left(\varepsilon^{2}\right)$ in equation (3.6.13) and equate terms of $O(1)$ yields, $l_{n}^{* 2}\left(\left(l_{n}^{*} \tau^{*}\right)^{2}-k_{0}^{* 4}\right)=0$. Therefore, looking only for real and positive wavenumbers,

$$
k_{0}^{*}=\sqrt{l_{n}^{*} \tau^{*}} .
$$

The corresponding dimensional wavelength $\tilde{\lambda}=\mathcal{L}_{\mathrm{t}} 2 \pi / k_{0}^{*}$ reads

$$
\tilde{\lambda}=\frac{2 \pi}{\sqrt{l_{n} \tau}}=2^{3 / 4}\left(\frac{\pi B}{n}\right)^{1 / 2}\left(\mathcal{L}_{\mathrm{f}}^{-2}+\mathcal{L}_{\mathrm{t}}^{-2}\right)^{-1 / 4},
$$

which is the same as equation (3.4.3) in the main text. For a higher order approximation, equate the $O(\varepsilon)$ terms of equation (3.6.13) to obtain

$$
2\left(l_{n}^{*} k_{1}^{*} k_{0}^{*}\right)^{2}+\left(k_{0}^{* 2}+l_{n}^{* 2}\right)^{2}\left(k_{0}^{* 2}+\tau^{* 2}\right)^{2}=0
$$

and hence,

$$
k_{1}^{*}=-\left(l_{n}^{*}+\tau^{*}\right)^{2} \sqrt{\frac{\tau^{*}}{4 l_{n}^{*}}} .
$$

Thus, the wavelength $\lambda^{*}=2 \pi / k^{*}$ for which $\omega^{*}$ is maximal is

$$
\lambda^{*}=\frac{2 \pi}{k_{0}^{*}+\varepsilon k_{1}^{*}+O\left(\varepsilon^{2}\right)}=\frac{2 \pi}{k_{0}^{*}}\left(1-\varepsilon \frac{k_{1}^{*}}{k_{0}^{*}}+O\left(\varepsilon^{2}\right)\right),
$$

which reads in dimensional form

$$
\mathcal{L}_{\mathrm{t}} \lambda^{*}=\frac{2 \pi}{\sqrt{l_{n} \tau}}+\frac{\Lambda_{\mathrm{eff}}}{a} \frac{\left(l_{n}+\tau\right)^{2}}{2 l_{n}} .
$$




\section{Oblique sand ridges in confined tidal channels}

T. M. Hepkema, H.E. de Swart, A. Nnafie, G. P. Schramkowski and H. M. Schuttelaars. Oblique sand ridges in confined tidal channels due to Coriolis and frictional torques. Ocean Dynamics, 70(12), 1505-1513 (2020). https://doi.org/10.1007/s10236-020-01413-0 


\begin{abstract}
The role of the Coriolis effect in the initial formation of bottom patterns in a tidal channel is studied by means of a linear stability analysis. The key finding is that the mechanism generating oblique tidal sand ridges on the continental shelf is also present in confined tidal channels. As a result, the Coriolis effect causes the fastest growing pattern to be a combination of tidal bars and oblique tidal sand ridges. Similar as on the continental shelf, the Coriolis-induced torques cause anticyclonic residual circulations around the ridges, which lead to the accumulation of sand above the ridges. Furthermore, an asymptotic analysis indicates that the maximum growth rate of the bottom perturbation is slightly increased by the Coriolis effect, while its preferred wavelength is hardly influenced.
\end{abstract}



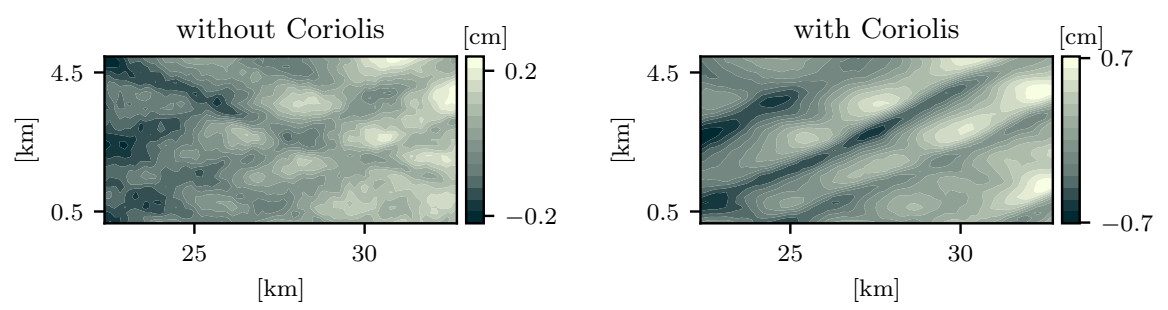

Figure 4.1: Bottom perturbation patterns obtained after approximately 15 years of morphodynamic simulation in a section of a semi-enclosed channel. In the left panel the Coriolis effect is neglected, whereas it is taken into account in the right panel. The simulations are done with the numerical model Delft3D in its depth-averaged mode (see online supplementary information).

\subsection{Introduction}

Tidal bars are rhythmic bottom patterns that occur in many tidal channels (e.g., the Western Scheldt in the Netherlands, the Exe Estuary in England, the Ord River Estuary in Australia and the Venice Lagoon in Italy). These bars are several meters high and have wavelengths of $1-15 \mathrm{~km}$. Their characteristics are determined by channel properties (depth, width, tidal amplitude, etc.), which may change due to, for example, dredging, sea level rise and land reclamation. Tidal bars are invaluable for many organisms that feed on their rich grounds, but they also may hamper marine traffic. For proper management of tidal channels, it is therefore important to understand their behavior.

Seminara and Tubino (2001), Schramkowski et al. (2002) and Chapter 3, among others, studied the physical mechanism that causes tidal bars to form, as well as the sensitivity of their wavelength to channel properties. They explained that the initial formation of tidal bars can be understood by analyzing the residual currents generated by the topography (using arguments similar to those by Zimmerman (1981)). Hibma et al. (2004) showed that the results of the linear stability analysis of Schramkowski et al. (2002) compare well with results of a numerical morphodynamic model, Delft3D.

In these linear stability studies and in the study by Hibma et al. (2004), the Coriolis effect was neglected. However, tidal bars occur in natural systems (e.g. Western Scheldt) where the Coriolis force is a first order term in the momentum balance. The importance of Coriolis on the hydro-morphodynamics in tidal channels is supported by several other studies, e.g., Valle-Levinson (2008), Winant (2008), Xie et al. (2017) and Olabarrieta et al. (2018). Furthermore, 2D morphological simulations similar (but now with and without the Coriolis effect) to those performed by Hibma et al. (2004) show clear differences between the initial formation of bottom patterns with and without 

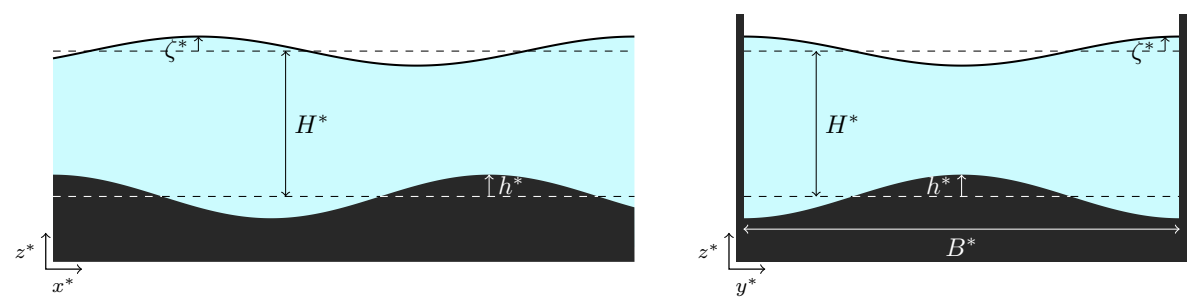

Figure 4.2: Model domain of semi-analytical model. Along-channel view (left). Crosschannel view (right). Here, $H^{*}$ is the undisturbed depth, $\zeta^{*}$ the free surface elevation, $h^{*}$ the bottom height and $B^{*}$ the channel width. The $x^{*}, y^{*}$ and $z^{*}$ arrows denote the direction of the coordinate axes $\left(x^{*}\right.$ is along-channel and $y^{*}$ cross-channel).

the Coriolis effect taken into account (see Figure 4.1 and online supplementary information). In the left panel of Figure 4.1, perturbations have a braided tidal bar pattern, whereas a ridge-like pattern emerges when the Coriolis effect is neglected (right panel).

This motivates an investigation into the physical mechanisms that explain how the initial formation of bottom patterns in a tidal channel is affected by the Coriolis effect. To this end, a semi-analytical model is developed in Section 4.2 and investigated by means of a linear stability analysis. In Section 4.3 the results of the linear stability analysis are presented and further analyzed with an asymptotic expansion considering a weak Coriolis force. Section 4.3 is followed by a discussion (Section 4.4) and the conclusions (Section 4.5).

\subsection{Model}

\subsubsection{Governing equations}

The semi-analytical model developed in this section is similar to those used by Schramkowski et al. (2002) and Chapter 3, which successfully explained the emergence of tidal bars in confined channels. The main extension here is that the Coriolis effect is taken into account.

The domain shape consists of an open section of a straight channel distant from the seaward and landward boundaries. The channel has a uniform width $B^{*}$, depth $H^{*}$ and a length which is small compared to the tidal wavelength and the length scale of channel width variations (Figure 4.2). These choices imply that we consider a local model, ignoring sloping background topography and apply the rigid-lid approximation (sea surface elevation only appears in the pressure gradient force). The hydrodynamics is governed by the depth-averaged shallow water equations, including the Coriolis effect. The flow is driven by a spatially uniform pressure gradient that oscillates with the principal tidal frequency $\sigma^{*}$. The dimensional (henceforth denoted with an asterisk) continuity 
and momentum equations read

$$
\begin{aligned}
\boldsymbol{\nabla}^{*} \cdot\left(\left(H^{*}-h^{*}\right) \boldsymbol{u}^{*}\right) & =0, \\
\frac{\partial \boldsymbol{u}^{*}}{\partial t^{*}}+\left(\boldsymbol{u}^{*} \cdot \boldsymbol{\nabla}^{*}\right) \boldsymbol{u}^{*}+\frac{r^{*} \boldsymbol{u}^{*}}{H^{*}-h^{*}}+\mathbf{F}^{*} \boldsymbol{u}^{*} & =-g^{*} \boldsymbol{\nabla} \zeta^{*} .
\end{aligned}
$$

Here, $t^{*}$ and $\boldsymbol{x}^{*}=\left(x^{*}, y^{*}\right)$ are the time and space coordinate. Furthermore, $\boldsymbol{\nabla}^{*}=\left(\partial / \partial x^{*}, \partial / \partial y^{*}\right), \boldsymbol{u}^{*}=\left(u^{*}, v^{*}\right)$ is the depth-averaged current velocity, $\zeta^{*}$ the free surface elevation and $h^{*}$ the bottom elevation (with respect to the undisturbed bed). In equation (4.2.2), the bottom stress is linearized (see e.g., Zimmerman, 1982, Terra et al., 2005), i.e., it is modelled as $\rho^{*} r^{*} \boldsymbol{u}^{*}$, where $\rho^{*}$ is the water density and $r^{*}=c_{\mathrm{d}} U^{*} 8 /(3 \pi)$, with $c_{\mathrm{d}}$ the drag coefficient and $U^{*}$ a typical amplitude of the tidal current in the along channel direction. The parameter $g^{*}$ is the gravitational acceleration and

$$
\mathbf{F}^{*}=f^{*}\left(\begin{array}{cc}
0 & -1 \\
1 & 0
\end{array}\right)
$$

with $f^{*}$ the Coriolis parameter (here assumed constant).

The bed elevation $h^{*}$ evolves due to the divergence of volumetric sediment transport $\boldsymbol{q}^{*}$,

$$
\left(1-p_{\star}\right) \frac{\partial h^{*}}{\partial t^{*}}=-\nabla^{*} \cdot \boldsymbol{q}^{*}
$$

Here, $p_{\star}$ is a porosity parameter and

$$
\boldsymbol{q}^{*}=s_{1}^{*}\left\|\boldsymbol{u}^{*}\right\|^{b_{1}} \boldsymbol{u}^{*}-s_{2}^{*}\left\|\boldsymbol{u}^{*}\right\|^{b_{2}} \nabla h^{*},
$$

where $\left\|\boldsymbol{u}^{*}\right\|^{2}=u^{* 2}+v^{* 2}$ and $s_{1}^{*}, s_{2}^{*}, b_{1}$ and $b_{2}$ are positive real numbers. The first term in equation (4.2.4) represents the advective transport of sediment and the second term accounts for bed slope effects. Equation (4.2.4) corresponds, with different choices for $s_{1}^{*}, s_{2}^{*}, b_{1}$ and $b_{2}$, to most bed load and total load sediment transport formulations (Soulsby, 1997).

The boundary conditions imposed at the sides of the channel are

$$
v^{*}=0 \text { and } \frac{\partial h^{*}}{\partial y^{*}}=0 \text { at } y^{*}=0, B^{*} .
$$

Equations (4.2.1)-(4.2.4) are made dimensionless (no asterisk) by first scaling time $t^{*}$ and space $\boldsymbol{x}^{*}$ as

$$
t=\sigma^{*} t^{*} \quad \text { and } \quad \boldsymbol{x}=\frac{\boldsymbol{x}^{*}}{B^{*}} .
$$

Subsequently, the depth-averaged velocity $\boldsymbol{u}^{*}=\left(u^{*}, v^{*}\right)$, the free surface elevation $\zeta^{*}$ and the bottom elevation $h^{*}$ are scaled as

$$
\boldsymbol{u}=\frac{\boldsymbol{u}^{*}}{U^{*}}, \quad \zeta=\frac{g^{*}}{U^{* 2}} \zeta^{*} \quad \text { and } \quad h=\frac{h^{*}}{H^{*}} .
$$


The dimensionless continuity and momentum equations read

$$
\begin{aligned}
\boldsymbol{\nabla} \cdot((1-h) \boldsymbol{u}) & =0 \\
\sigma \frac{\partial \boldsymbol{u}}{\partial t}+(\boldsymbol{u} \cdot \boldsymbol{\nabla}) \boldsymbol{u}+\frac{r \boldsymbol{u}}{1-h}+\mathbf{F} \boldsymbol{u}+\boldsymbol{\nabla} \zeta & =0
\end{aligned}
$$

where $\boldsymbol{\nabla}=(\partial / \partial x, \partial / \partial y), \sigma=\sigma^{*} B^{*} / U^{*}$, $r=r^{*} B^{*} /\left(U^{*} H^{*}\right)$ and

$$
\mathbf{F}=\frac{B^{*}}{U^{*}} \mathbf{F}^{*}=\frac{1}{R o}\left(\begin{array}{cc}
0 & -1 \\
1 & 0
\end{array}\right),
$$

with $R o=U^{*} /\left(f^{*} B^{*}\right)$ the Rossby number.

The dimensionless bed elevation equation reads

$$
\frac{\partial h}{\partial t}=-\varepsilon \boldsymbol{\nabla} \cdot\left(\|\boldsymbol{u}\|^{b_{1}} \boldsymbol{u}-\tilde{\Lambda}\|\boldsymbol{u}\|^{b_{2}} \nabla h\right) .
$$

Here, $\tilde{\Lambda}=s_{2}^{*} U^{* b_{2}-\left(b_{1}+1\right)} H^{*} /\left(s_{1}^{*} B^{*}\right)$ is a bed slope parameter and $\varepsilon$ the ratio of the tidal time scale $1 / \sigma^{*}$ and the morphological time scale $\left(1-p_{\star}\right) H^{*} B^{*} / Q^{*}$, with $Q^{*}=s_{1}^{*} U^{* b_{1}+1}$ a typical volumetric sediment transport magnitude. Given that the bed evolves slowly and that the sediment transport varies almost periodically (with the tidal frequency), the bed evolution is approximated by the tidal average of the divergence of the sediment transport,

$$
\frac{\partial h}{\partial \tau}=-\langle\nabla \cdot \boldsymbol{q}\rangle \quad \text { with } \quad \boldsymbol{q}=\|\boldsymbol{u}\|^{b_{1}} \boldsymbol{u}-\tilde{\Lambda}\|\boldsymbol{u}\|^{b_{2}} \nabla h
$$

where $\tau=\varepsilon t$ and $\langle\cdot\rangle$ denotes the average over one dimensionless tidal period $2 \pi$.

The dimensionless boundary conditions read

$$
v=\frac{\partial h}{\partial y}=0 \quad \text { at } \quad y=0,1 .
$$

\subsubsection{Linear stability analysis}

The bottom pattern that initially forms when a tidal current flows over a horizontal sandy bed, is analysed with a linear stability analysis. An equilibrium solution $\left(u_{0}, v_{0}, \zeta_{0}, h_{0}\right)$ to the system of equations $(4.2 .6)-(4.2 .9)$, is described by a spatially uniform symmetrical tidal current $\boldsymbol{u}_{0}=\left(u_{0}, v_{0}\right)=(\cos (t), 0)$, driven by a spatially uniform pressure gradient

$$
-\nabla \zeta_{0}=\sigma \frac{\partial \boldsymbol{u}_{0}}{\partial t}+r \boldsymbol{u}_{0}+\mathbf{F} \boldsymbol{u}_{0}
$$

over a horizontal bed $h_{0}=0$. 
Small perturbations on the flat bed result in perturbations of the flow variables and vice versa. Let $\xi$ be small and substitute

$$
\begin{aligned}
h & =h_{0}+\xi h_{1}+O\left(\xi^{2}\right), \\
\boldsymbol{u} & =\boldsymbol{u}_{0}+\xi \boldsymbol{u}_{1}+O\left(\xi^{2}\right), \\
\zeta & =\zeta_{0}+\xi \zeta_{1}+O\left(\xi^{2}\right)
\end{aligned}
$$

in equations (4.2.6)-(4.2.8). At $O(\xi)$ this results in a linear system of partial differential equations,

$$
\begin{aligned}
\frac{\partial u_{1}}{\partial x}+\frac{\partial v_{1}}{\partial y}-u_{0} \frac{\partial h_{1}}{\partial x} & =0 \\
\sigma \frac{\partial u_{1}}{\partial t}+u_{0} \frac{\partial u_{1}}{\partial x}+r\left(u_{1}+u_{0} h_{1}\right)-\frac{v_{1}}{R o}+\frac{\partial \zeta_{1}}{\partial x} & =0 \\
\sigma \frac{\partial v_{1}}{\partial t}+u_{0} \frac{\partial v_{1}}{\partial x}+r v_{1}+\frac{u_{1}}{R o}+\frac{\partial \zeta_{1}}{\partial y} & =0 \\
\frac{\partial h_{1}}{\partial \tau}+b_{1}\left\langle\left|u_{0}\right|^{b_{1}} \frac{\partial u_{1}}{\partial x}\right\rangle-\Lambda \nabla^{2} h_{1} & =0 .
\end{aligned}
$$

Here, $\Lambda=\tilde{\Lambda}\left\langle\left|u_{0}\right|^{b_{2}}\right\rangle$ is a bed slope parameter and $u_{1}$ and $v_{1}$ are the components of $\boldsymbol{u}_{1}=\left(u_{1}, v_{1}\right)$. In the derivation of equation (4.2.13) the continuity equation (4.2.10) and $\left\langle\left|u_{0}\right|^{b_{1}} u_{0}\right\rangle=0$ are used to simplify the expression. Combining the continuity and momentum equations (substituting (4.2.10) in $\partial / \partial x(4.2 .12)-\partial / \partial y(4.2 .11))$ eliminates $\zeta_{1}$ and yields an equation for the vorticity $\Omega_{1}$ :

$$
\sigma \frac{\partial \Omega_{1}}{\partial t}+u_{0} \frac{\partial \Omega_{1}}{\partial x}+r \Omega_{1}=u_{0}\left(r \frac{\partial h_{1}}{\partial y}-\frac{1}{R o} \frac{\partial h_{1}}{\partial x}\right)
$$

with

$$
\Omega_{1}=\frac{\partial v_{1}}{\partial x}-\frac{\partial u_{1}}{\partial y} .
$$

The first term on the right hand side of equation (4.2.14) is the torque due to bottom friction. The second term on the right hand side is the Coriolis torque. The terms on the left hand side of equation (4.2.14) describe the vorticity's inertia, its advection by the equilibrium current $u_{0}$ and its dissipation due to bottom friction, respectively.

Equations (4.2.10), (4.2.13), (4.2.14) and (4.2.15), together with the boundary conditions, allow for solutions of the form

$$
\left(u_{1}, v_{1}, \Omega_{1}, h_{1}\right)=(\hat{u}, \hat{v}, \hat{\Omega}, \hat{h}) e^{\mathrm{i} k x}+\text { c.c. }
$$

Here, c.c. stands for complex conjugate, $\hat{u}(t, y, k), \hat{v}(t, y, k), \hat{\Omega}(t, y, k), \hat{h}(\tau, y, k)$ are complex valued functions and $k$ is a dimensionless along-channel wavenumber, which relates to a dimensional wavenumber $k^{*}=k / B^{*}$. Substituting 
(4.2.16) in the continuity equation (4.2.10) and the vorticity equation (4.2.14) yields

$$
\begin{aligned}
\mathrm{i} k \hat{u}+\frac{\partial \hat{v}}{\partial y} & =u_{0} \mathrm{i} k \hat{h} \\
\frac{\sigma}{r} \frac{\partial \hat{\Omega}}{\partial t}+\left(\frac{u_{0} \mathrm{i} k}{r}+1\right) \hat{\Omega} & =u_{0}\left(\frac{\partial \hat{h}}{\partial y}-\gamma \mathrm{i} k \hat{h}\right) .
\end{aligned}
$$

The parameter $\gamma=(r R o)^{-1}$ denotes the relative importance of the vorticity producing torque due to the Coriolis effect and the torque due to the bottom friction. Thus, when the Coriolis effect is neglected, $\gamma=0$ and only the torque due to bottom friction produces vorticity. Substituting (4.2.16) in the bottom evolution equation $(4.2 .13)$ results in

$$
\frac{\partial \hat{h}}{\partial \tau}=-b_{1} \mathrm{i} k\left\langle\left|u_{0}\right|^{b_{1}} \hat{u}\right\rangle+\Lambda\left(\frac{\partial^{2} \hat{h}}{\partial y^{2}}-k^{2} \hat{h}\right) .
$$

Inspired by the boundary conditions and the fact that without the Coriolis effect, $\hat{h} \sim \cos (n \pi y)$ with $n$ a natural number (Schramkowski et al., 2002), we write $\hat{h}$ as a cosine series,

$$
\hat{h}=\sum_{n=0}^{\infty} \tilde{h}_{n} \cos (n \pi y),
$$

with $\tilde{h}_{n}(\tau, k)$ complex valued functions for every natural number $n$. In appendix 4.6.1, the current $\hat{u}$ is expressed in terms of the bottom elevation $\hat{h}$ by solving (4.2.17)-(4.2.18). Furthermore, it is shown in the appendix that substituting the cosine series (4.2.20) into the bottom evolution equation (4.2.19) and truncating the summation at $N$ results in

$$
\frac{\partial \tilde{\boldsymbol{h}}}{\partial \tau}=(\mathbf{D}+\gamma \mathbf{A}) \tilde{\boldsymbol{h}} .
$$

Here, $\tilde{\boldsymbol{h}}=\left(\tilde{h}_{0}, \ldots, \tilde{h}_{N}\right), \mathbf{D}=\operatorname{diag}\left(\omega_{0}^{0}, \ldots, \omega_{N}^{0}\right)$ is a diagonal matrix and $\mathbf{A}$ is a matrix with elements $a_{m n}$, where

$$
\begin{aligned}
\omega_{m}^{0} & =\alpha \frac{(m \pi)^{2}}{k^{2}+(m \pi)^{2}}-\Lambda\left((m \pi)^{2}+k^{2}\right), \\
a_{m n} & = \begin{cases}0 & \text { if } m+n \text { is even, } \\
\frac{4 \alpha i k m^{2}}{\left(m^{2}-n^{2}\right)\left((m \pi)^{2}+k^{2}\right)} & \text { otherwise, }\end{cases}
\end{aligned}
$$

with $\alpha=b_{1} \mathrm{i} k\left\langle\left|u_{0}\right|^{b_{1}} \varphi\right\rangle$ a positive real number, where $\varphi(t)$ is the time-dependent part of $\hat{\Omega}$ (see Appendix 4.6.1). Equation (4.2.21) admits solutions of the form

$$
\tilde{\boldsymbol{h}}=e^{\omega \tau} \boldsymbol{h}
$$




\begin{tabular}{llll}
\hline parameter & value & definition & description \\
\hline$\sigma$ & 0.7 & $\sigma^{*} B^{*} / U^{*}$ & relative importance of inertia to advection \\
$r$ & 1 & $r^{*} B^{*} /\left(U^{*} H^{*}\right)$ & relative importance of friction to advection \\
$R o^{-1}$ & 0.5 & $f^{*} B^{*} / U^{*}$ & relative importance of Coriolis to advection \\
$\Lambda$ & 0.005 & $\tilde{\Lambda}\left\langle\left|u_{0}\right|^{\left.b_{2}\right\rangle}\right.$ & bed slope parameter \\
$b_{1}$ & 2 & & advective sediment transport parameter \\
$b_{2}$ & 2 & & bed slope effect parameter \\
$N+1$ & 10 & & number of terms in cosine series of $\hat{h}$ \\
\hline
\end{tabular}

Table 4.1: Model parameters and their default values. These values are representative for the Western Scheldt (values for the dimensional parameters are given in the text).

with $\omega$ an eigenvalue of $\mathbf{D}+\gamma \mathbf{A}$ and $\boldsymbol{h}$ the corresponding eigenvector. The superscript zero of the elements in the diagonal matrix $\mathbf{D}$ represents the fact that these are the eigenvalues when $\gamma=0$ (no Coriolis effect).

For every wavenumber $k$, we calculate the eigenvector $\boldsymbol{h}_{j}(k)$ corresponding to the $j$-th eigenvalue $\omega_{j}(k)$. The eigenvalues and corresponding eigenvectors are sorted such that $\omega_{0} \geq \cdots \geq \omega_{N}$. The wavenumber $k$ for which the largest growth rate $\omega_{0}(k)$ is attained, is called the preferred wavenumber $k_{\text {pref }}$. The corresponding eigenvector $\boldsymbol{h}_{0}\left(k_{\text {pref }}\right)=\left(p_{0}, \ldots, p_{N}\right)$, sets the spatial structure of the fastest growing bottom pattern:

$$
h_{\text {pref }}=\sum_{n=0}^{N} p_{n} \cos (n \pi y) e^{\mathrm{i} k_{\text {pref }} x}+\text { c.c. }
$$

The second and third largest eigenvalues are denoted by $\omega_{1}$ and $\omega_{2}$, respectively. The dimensionless growth rates $\omega$ relate to dimensional ones by $\omega^{*}=\omega \varepsilon \sigma^{*}$ (since $\tau=\varepsilon t=\varepsilon \sigma^{*} t^{*}$ ).

\subsection{Results}

In the following experiments, we chose model parameters based on the Western Scheldt. The dimensionless model parameters are summarized in Table 4.1. They correspond to a channel width of $B^{*}=5 \mathrm{~km}$, channel depth of $H^{*}=$ $10 \mathrm{~m}$ and with a typical current velocity with amplitude $U^{*}=1 \mathrm{~ms}^{-1}$ and radian frequency $\sigma^{*}=1.4 \cdot 10^{-4} \mathrm{~s}^{-1}\left(\mathrm{M}_{2}\right.$ tide). The gravitational constant is $g^{*}=10 \mathrm{~m} \mathrm{~s}^{-2}$, the friction parameter $r^{*}=2 \cdot 10^{-3} \mathrm{~ms}^{-1}$ and the (default) Coriolis parameter $f^{*}=10^{-4} \mathrm{~s}^{-1}$. The sediment transport parameters are (following Schramkowski et al. (2002) and Chapter 3), $s_{1}^{*}=3 \cdot 10^{-4} \mathrm{~m}^{1-b_{1}} \mathrm{~s}^{b_{1}}$, $s_{2}^{*}=1.5 \cdot 10^{-3} \mathrm{~m}^{2-b_{2}} \mathrm{~s}^{b_{2}-1}, b_{1}=b_{2}=2$ (see Section 4.4.2) and porosity $p_{\star}=0.4$. 

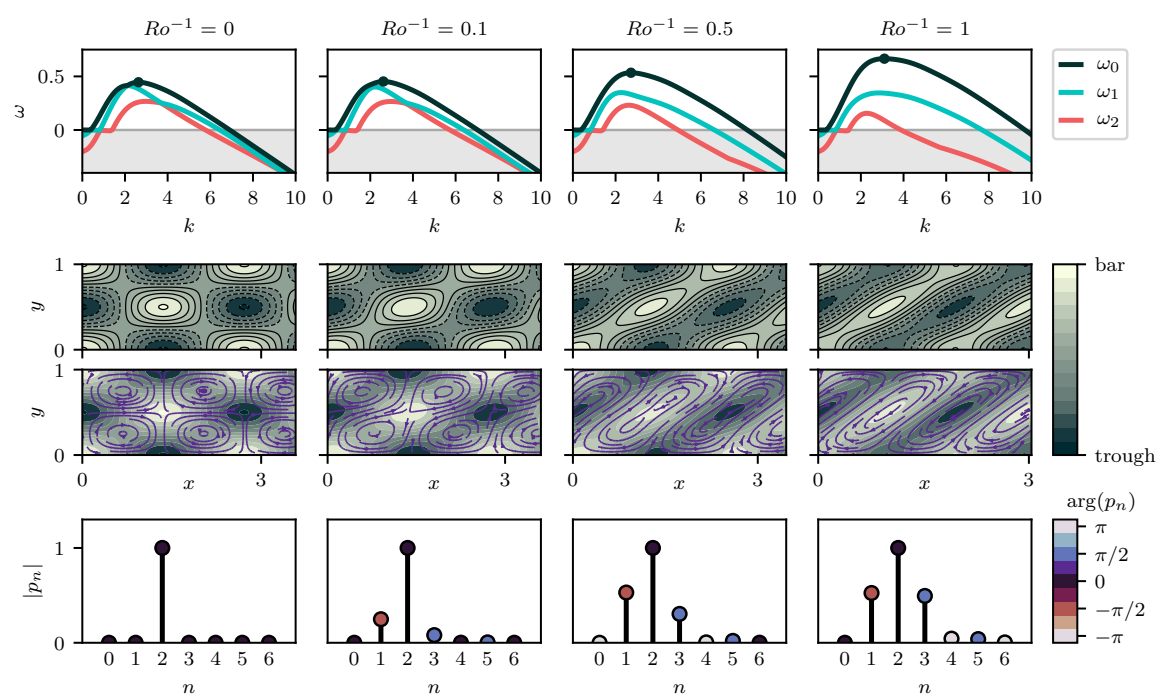

Figure 4.3: Top row: dimensionless growth rates of the three fastest growing modes, $\omega_{0}, \omega_{1}$ and $\omega_{2}$, versus dimensionless along-channel wavenumber $k$ for different values of the inverse Rossby number $R^{-1}$. The dots in the top panels indicate the maximum growth rate and corresponds to the figures in the rows below. Second row: fastest growing bottom pattern for different values of $R^{-1}$. Third row: residual currents over the bottom pattern in the second row. Bottom row: amplitudes and arguments of cosine series coefficients that correspond to the the bottom patterns in the second and third row (see equation (4.2.25)). A value $R o^{-1}=0.5$ is representative for the Western Scheldt. A dimensionless growth rate $\omega=0.5$ corresponds to a dimensional growth rate of approximately $0.16 \mathrm{yr}^{-1}$ and a dimensionless wavelength $2 \pi / k=3$ corresponds to a dimensional wavelength of $15 \mathrm{~km}$. 


\subsubsection{Sensitivity to Rossby number}

To investigate the role of the Coriolis effect in the initial formation of bars, the inverse Rossby number $R o^{-1}$ is varied. All other parameters are kept fixed such that varying $R o^{-1}$ corresponds to varying the Coriolis parameter $f^{*}$. The results are summarized in Figure 4.3. The top panels of the figure show the first three growth curves (i.e., $\omega_{0}, \omega_{1}$ and $\omega_{2}$ versus wavenumber $k$ ) for different values of the inverse Rossby number $R o^{-1}$. The figure reveals that the growth rate of the fastest growing pattern slightly increases with increasing $R o^{-1}$. The wavenumber $k_{\text {pref }}$ for which the growth rate attains its maximum value is hardly influenced by the Coriolis effect.

The panels in the two middle rows in Figure 4.3 illustrate the patterns and the residual current that correspond to the fastest growing patterns for different values of the inverse Rossby number. When the Coriolis effect is neglected $\left(R o^{-1}=0\right)$, the patterns have a tidal bar structure of $\cos (n \pi y) \cos (k x)$, with $n$ a natural number and $k$ the wavenumber. However, the pattern significantly changes when the Coriolis effect is considered. In that case, the fastest growing pattern seems a combination of tidal bars and oblique tidal ridges. Moreover, when the Coriolis effect is neglected or very weak, the cells of residual current are in between troughs and bars, whereas the residual currents go around the bars and troughs when the Coriolis effect is taken into account.

The bottom row of panels in Figure 4.3 shows, for different values of the inverse Rossby number $R o^{-1}$, the components $p_{n}$ of the eigenvector $\boldsymbol{h}_{0}\left(k_{\text {pref }}\right)$. The figure reveals that the spectrum widens with increasing $R o^{-1}$. Moreover, looking at the phases of the components $p_{n}$, it appears that in all cases considered, the patterns are to a good approximation of the form

$$
\begin{aligned}
h_{\text {pref }} & \approx 2\left|p_{1}\right| \cos (\pi y) \sin \left(k_{\text {pref }} x\right) \\
& +2\left|p_{2}\right| \cos (2 \pi y) \cos \left(k_{\text {pref }} x\right) \\
& -2\left|p_{3}\right| \cos (3 \pi y) \sin \left(k_{\text {pref }} x\right) .
\end{aligned}
$$

The fact that only a narrow part of the spectrum is involved follows from expression (4.2.23). Since the coefficients $a_{m n}$ decrease when $\left(m^{2}-n^{2}\right)$ increases, the equation of $\partial \tilde{h}_{m} / \partial \tau$ is mostly dependent on the coefficients $\tilde{h}_{n}$ for which $n$ is close to $m$.

\subsubsection{Asymptotic analysis for weak Coriolis force}

Above, it was shown that when the Coriolis effect is considered, the maximum growth rate slightly increases and the fastest growing pattern changes significantly. An explanation for this is sought by analyzing the system for small values of $\gamma$ (while fixing $r$ ), which corresponds to small values of the inverse Rossby number $R o^{-1}$ (i.e., weak Coriolis force). 


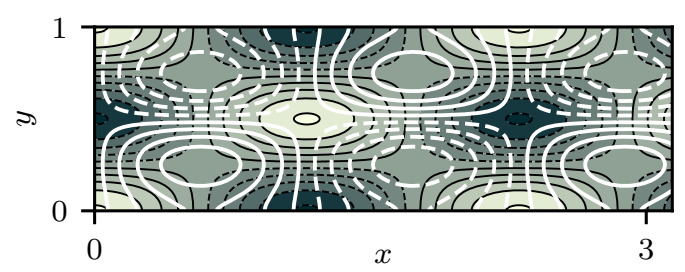

Figure 4.4: The $O(1)$ (as $\gamma \downarrow 0$ ) bottom pattern (colors). The white contour lines denote the $O(\gamma)$ correction due to the Coriolis effect. Solid lines denote positive values (bar) and dashed lines negative ones (troughs).

Let, as before, $\omega_{j}$ and $\boldsymbol{h}_{j}$ for $j=0, \ldots N$ be eigenvalues and eigenvectors of the (perturbed) eigenvalue problem

$$
\omega \boldsymbol{h}=(\mathbf{D}+\gamma \mathbf{A}) \boldsymbol{h}
$$

and expand these eigenvalues and eigenvectors in powers of $\gamma$ :

$$
\begin{aligned}
& \omega_{j}=\omega_{j}^{0}+\gamma \omega_{j}^{1}+\gamma^{2} \omega_{j}^{2}+O\left(\gamma^{3}\right), \\
& \boldsymbol{h}_{j}=\boldsymbol{h}_{j}^{0}+\gamma \boldsymbol{h}_{j}^{1}+\gamma^{2} \boldsymbol{h}_{j}^{2}+O\left(\gamma^{3}\right),
\end{aligned}
$$

for $j=0, \ldots, N$. The eigenvectors of the $O(1)$ eigenvalue problem are $\boldsymbol{h}_{j}^{0}=\boldsymbol{e}_{j}$ with $\boldsymbol{e}_{j}$ the standard basis vectors of $\mathbb{R}^{N+1}$ (i.e., the $j$-th entry of $\boldsymbol{e}_{j}$ equals one and the others zero). The eigenvalues $\omega_{j}^{0}$ are given in (4.2.22).

At $O(\gamma)$, equation (4.3.1) reads

$$
\omega_{j}^{0} \boldsymbol{h}_{j}^{1}+\omega_{j}^{1} \boldsymbol{e}_{j}=\mathbf{D} \boldsymbol{h}_{j}^{1}+A \boldsymbol{e}_{j}
$$

The $O(\gamma)$ correction to the eigenvalues is now computed by taking the standard inner product of (4.3.2) with $\boldsymbol{e}_{j}$, resulting in

$$
\omega_{j}^{1}=a_{j j} .
$$

Here, it is used that $\omega_{j}^{0} \boldsymbol{h}_{j}^{1} \cdot \boldsymbol{e}_{j}=\mathbf{D} \boldsymbol{h}_{j}^{1} \cdot \boldsymbol{e}_{j}$. Given that $a_{j j}=0$, it follows that there is no $O(\gamma)$ correction to the eigenvalues. However, there is an $O(\gamma)$ correction to the eigenvectors. To see this, take the inner product of (4.3.2) with $\boldsymbol{e}_{m}(m \neq j)$ to obtain the $m$-th component of $\boldsymbol{h}_{j}^{1}$,

$$
\boldsymbol{h}_{j}^{1} \cdot \boldsymbol{e}_{m}=\frac{a_{m j}}{\omega_{j}^{0}-\omega_{m}^{0}}
$$

Figure 4.4 shows that the the superposition of the tidal bar pattern (colors) and the perturbation induced by the Coriolis effect (white contour lines) results in the tidal sand ridges by connecting the bars and troughs. Since the perturbation 

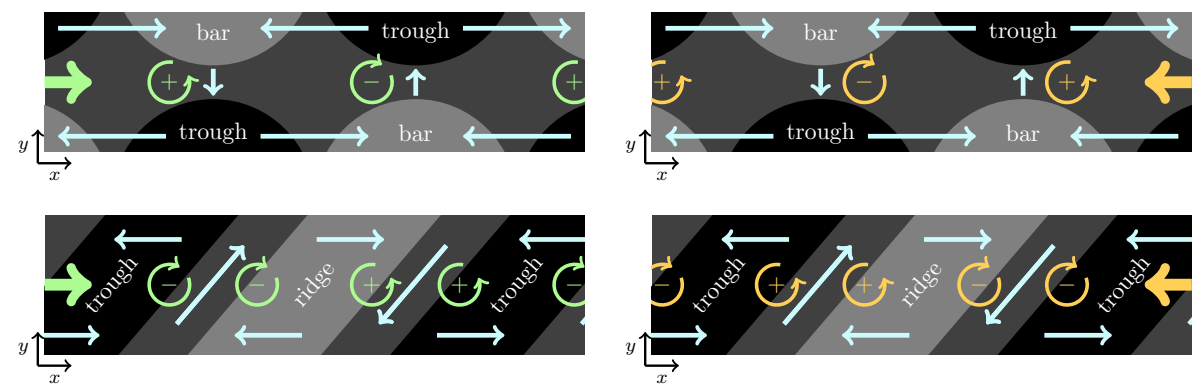

Figure 4.5: The effect of bottom perturbations in the form of tidal bars (top panels) and of tidal sand ridges (bottom panels) on the tidal current. The left panel depicts the situation during flood (fat green arrow) and the right panel the one during ebb (fat orange arrow). The green arrows and orange round arrows denote the vorticity at the principle tidal frequency $\mathrm{M}_{2}$. The light blue arrows depicts the residual tidal vorticity. (After Zimmerman (1981) and Chapter 3).

due to the Coriolis effect is anti-symmetric with respect to the channel axes, it breaks the reflective symmetry of the tidal bar pattern (with respect to the middle of the channel).

Lastly, we show that the correction to the largest eigenvalue is positive by considering the $O\left(\gamma^{2}\right)$ problem,

$$
\omega_{j}^{0} \boldsymbol{h}_{j}^{2}+\omega_{j}^{1} \boldsymbol{h}_{j}^{1}+\omega_{j}^{2} \boldsymbol{e}_{j}=\mathbf{D} \boldsymbol{h}_{j}^{2}+A \boldsymbol{h}_{j}^{1} .
$$

Taking the inner product with $\boldsymbol{e}_{j}$ and substituting $\omega_{j}^{1}=0$ results in

$$
\begin{aligned}
\omega_{j}^{2} & =A \boldsymbol{h}_{j}^{1} \cdot \boldsymbol{e}_{j}=\sum_{m=1}^{N}\left(\boldsymbol{h}_{j}^{1} \cdot \boldsymbol{e}_{m}\right)\left(A \boldsymbol{e}_{m} \cdot \boldsymbol{e}_{j}\right) \\
& =\sum_{m=1}^{N} \frac{a_{m j} a_{j m}}{\omega_{j}^{0}-\omega_{m}^{0}}
\end{aligned}
$$

where again, it is used that $\omega_{j}^{0} \boldsymbol{h}_{j}^{2} \cdot \boldsymbol{e}_{j}=\mathbf{D} \boldsymbol{h}_{j}^{2} \cdot \boldsymbol{e}_{j}$. For all $m, a_{m j} a_{j m}>0$ and if $\omega_{j}^{0}$ is the largest eigenvalue of the unperturbed system, $\omega_{j}^{0}-\omega_{m}^{0}>0$. Hence, a weak Coriolis effect increases the maximum growth rate of the bottom perturbations, which is consistent with the findings in Figure 4.3.

\subsection{Discussion}

\subsubsection{Oblique tidal ridges versus tidal bars}

The differences in patterns when the Coriolis effect is taken into account or not, follow from the additional torque exerted by the Coriolis effect on water motion over the longitudinally sloping bed. When the Coriolis effect is neglected 
and only the frictional torque due to lateral bottom slopes is considered, the fastest growing bottom patterns consist of tidal bar patterns, identical to those obtained by Seminara and Tubino (2001), among others. An elaborate explanation of the physical mechanism of the formation of tidal bars is given in Chapter 3. When the Coriolis torque is taken into account, the joint action of the two torques results in the formation of oblique tidal sand ridges. This mechanism is the same as the one responsible for the initial formation of offshore tidal sand ridges, as explained by Huthnance (1982). For completeness, we summarize the two mechanisms below.

Both the morphodynamic instability leading to tidal bars as the instability leading to tidal sand ridges result from the fact that perturbations of the flat bottom in the form of tidal bars or tidal sand ridges alter the tidal currents flowing over them (or vice versa). This tide-topography interaction results in residual currents, such that both during the ebb and flood phase, the currents become stronger upstream of the bars/ridges, whereas they are weakened downstream of the bars/ridges (and vice versa for the troughs) (Zimmerman, 1981). This results in sediment transport converging at the bars or ridges and diverging at the troughs, hence, the instability.

Figure 4.5 illustrates the tide-topography interaction. In the top panels a tidal bar pattern is considered with a tidal current flowing over it. Due to lateral gradients in the bottom, a frictional torque generates vorticity at the principal tidal frequency, between the bars and troughs (green round arrows in the figure). The $\mathrm{M}_{2}$ vorticity is transported by the background $\mathrm{M}_{2}$ current, resulting in a residual tidal vorticity as indicated by the light blue arrows in the figure. Adding the background current to the residual current results in higher velocities upstream of a bar and lower velocities downstream of the bar (and vice versa for the troughs).

In the bottom panels of Figure 4.5 a tidal sand ridge pattern is shown with the same background current. Also here, due to lateral bottom slopes, a frictional torque generates vorticity at the $\mathrm{M}_{2}$ frequency. Now, in addition, the longitudinal bottom slopes result in a Coriolis torque. On the Northern Hemisphere, the Coriolis and frictional torque are in the same direction when the ridges are rotated anti-clockwise with respect to the background current. Also in this case, the background $\mathrm{M}_{2}$ current transports the $\mathrm{M}_{2}$ vorticity, resulting in residual vorticity as indicated by the light blue arrows in the figure. Again, when the residual current is added to the background current the velocities are higher upstream of the ridge than downstream and vice versa for the troughs.

An essential difference between the residual currents due to tidal bars and those due to tidal ridges, is that in the later case the residual currents are around the crests, whereas in the former case they are directed towards the crests. Note that pure tidal ridges as in the figure can not form in a confined channel, because they violate the lateral boundary conditions, but a similar pattern is possible. 

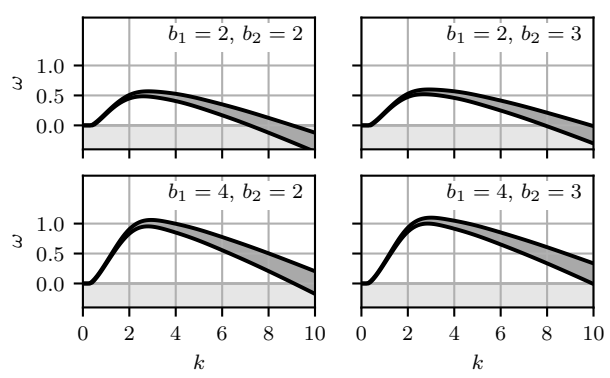

Figure 4.6: Dimensionless growth rates of the fastest growing mode $\omega_{0}$, versus dimensionless along-channel wavenumber $k$ for different values of the sediment transport parameters $b_{1}$ and $b_{2}$. Here, $R o^{-1}=0.5$ and the values of $s_{1}^{*}$ and $s_{2}^{*}$ are their default values $\pm 10 \%$ (shaded area).

\subsubsection{Sediment transport formulation}

In the sediment transport formulation $\boldsymbol{q}$ in equation (4.2.8) we chose $b_{1}=b_{2}=$ 2. This corresponds to advective bed load transport as in Bailard (1981) and a bed slope effect due to eddy diffusivity, which is larger than the bed load bed slope effect as in the Bailard formulation (see Chapter 3). Following the later study, the bed slope parameter $s_{2}^{*}$ is calculated as

$$
s_{2}^{*}=\frac{\mu^{*} w_{s}^{*} \alpha^{*}}{\kappa_{v}^{*} \gamma^{*}}\left(1-e^{-\frac{w_{s}^{*}}{\kappa_{v}^{*} H^{*}}}\right)^{-1} \approx 0.0015 \mathrm{~s}
$$

where $\mu^{*}\left(\approx 5 \mathrm{~m}^{2} \mathrm{~s}^{-1}\right)$ is the horizontal eddy diffusion coefficient, $w_{s}^{*}(\approx$ $\left.0.013 \mathrm{~m} \mathrm{~s}^{-1}\right)$ the sediment settling velocity, $\kappa_{v}^{*}\left(\approx 0.01 \mathrm{~m}^{2} \mathrm{~s}^{-1}\right)$ the vertical eddy diffusivity coefficient, $\alpha^{*}\left(\approx 4 \cdot 10^{-6} \mathrm{~s} \mathrm{~m}^{-1}\right)$ an erosion parameter and $\gamma^{*}$ $\left(\approx 0.017 \mathrm{~s}^{-1}\right)$ a deposition parameter.

When $b_{1}=2$ and $b_{2}=3$, the transport is the same as for the Bailard formulation for bed load sediment transport (Bailard, 1981). When $b_{1}=4$, advective transport is similar to the Engelund Hansen parametrization for total load transport (Engelund and Hansen, 1967).

The magnitude of the dimensionless growth rate depends on the choice of $b_{1}$ and $b_{2}$ (see Figure 4.6), but the shape of the fastest growing patterns is hardly affected by this choice (see supplementary information). When $b_{1}=4$ the growth rates increase. When $b_{2}=3$ the differences are minimal for $k \approx k_{\text {pref }}$. For larger wavenumbers the growth rate is smaller when $b_{2}=3$ compared to $b_{2}=2$, due to an increased bed slope effect. However, for those wavenumbers advection of suspended sediment becomes relevant and the total load sediment transport formulation (4.2.4) is no longer valid (see Chapter 3). 


\subsubsection{Comparison with a numerical model}

The results of Section 4.3, obtained with the semi-analytical model, correspond qualitatively with those of the numerical model that has a similar set-up as the one from Hibma et al. (2004), but includes the Coriolis effect (see online SI). When the Coriolis effect is taken into account, oblique sand ridges also form in this numerical model. Moreover, their growth rate is larger than that of the tidal bars that form in the absence of Coriolis (see the color bars in Figure 4.1), whilst their wavelength is hardly affected.

The wavelengths of the tidal bars in Figure 4.1 produced with the numerical model are approximately $5-7 \mathrm{~km}$. The average depth in the area considered is $6.5 \mathrm{~m}$ and the current velocities amplitudes are close to $1.0 \mathrm{~m} \mathrm{~s}^{-1}$. In the semi-analytical model, when $H^{*}=6.5 \mathrm{~m}$ and $U^{*}=1 \mathrm{~ms}^{-1}$, the preferred wavenumber is $k_{\text {pref }} \approx 3.6$, corresponding to dimensional wavelengths of approximately $8.7 \mathrm{~km}$. Hence, the wavelength of the semi-analytical model is slightly larger, but in the same order of magnitude as those simulated by the numerical model. The similarities between the two different models confirm robustness of the discussed mechanism.

\subsection{Conclusions}

To study the role of the Coriolis effect in the initial formation of bottom patterns in a tidal channel, a semi-analytical model is extended to include the Coriolis effect. It was shown that the Coriolis effect breaks the (anti-)symmetry of the bottom pattern that initially forms. The fastest growing bed perturbation can be characterized as a combination of tidal bars and oblique ridges, unlike the case without Coriolis effect where only tidal bars form. The Coriolis effect also modifies the residual current so that it drives anticyclonic circulations around the ridges. The mechanism behind these modifications is the same as the one causing the formation of oblique tidal sand ridges on the continental shelf. Compared to the case where the Coriolis effect is neglected, the preferred wavenumber is similar, while the maximum growth rate slightly increases.

\subsection{Appendix}

\subsubsection{Derivation of bed evolution equation}

The current $\hat{u}$ in equation (4.2.19) is eliminated by first solving the vorticity equation (4.2.18). Homogeneous solutions decay due to the bottom friction. Therefore, a nontransient particular solution reads

$$
\hat{\Omega}=\mathrm{i} k \hat{v}-\frac{\partial \hat{u}}{\partial y}=\varphi\left(\frac{\partial \hat{h}}{\partial y}-\gamma \mathrm{i} k \hat{h}\right) .
$$


Here, $\varphi(t)$ is the time dependent part of $\hat{\Omega}$, which is found by solving

$$
\frac{\sigma}{r} \frac{\partial \varphi}{\partial t}+\left(\frac{u_{0} \mathrm{i} k}{r}+1\right) \varphi=u_{0}
$$

for each harmonic component by means of a truncated Fourier series. Multiplying equation (4.6.1) by $\mathrm{i} k$ and adding it to the $\partial / \partial y$ of equation (4.2.17) results in a differential equation for $\hat{v}$,

$$
\frac{\partial^{2} \hat{v}}{\partial y^{2}}-k^{2} \hat{v}=\underbrace{\mathrm{i} k\left(\left(\varphi+u_{0}\right) \frac{\partial \hat{h}}{\partial y}-\gamma \varphi \mathrm{i} k \hat{h}\right)}_{F}
$$

with boundary conditions $\hat{v}=0$ at $y=0$ and $y=1$. Variation of parameters yields a solution for $\hat{v}$ and $\partial \hat{v} / \partial y$,

$$
\hat{v}=\int_{0}^{1} G(y, s) F(t, s) \mathrm{d} s \text { and } \frac{\partial \hat{v}}{\partial y}=\int_{0}^{1} \frac{\partial G}{\partial y}(y, s) F(t, s) \mathrm{d} s,
$$

with $F(t, y)$ the right hand side of (4.6.2) and Green's function

$$
G(y, s)= \begin{cases}\frac{-\sinh (k(1-y)) \sinh (k s)}{k \sinh (k)} & \text { if } s<y, \\ \frac{-\sinh (k y) \sinh (k(1-s))}{k \sinh (k)} & \text { if } s>y .\end{cases}
$$

The current $\hat{u}$ in terms of $\hat{h}$, now follows from the continuity equation (4.2.17). Multiplying $\hat{u}$ by $\left|u_{0}\right|^{b_{1}}$, taking the tidal average and substituting this in equation (4.2.19), results in the bed evolution equation

$$
\frac{\partial \hat{h}}{\partial \tau}=\alpha \int_{0}^{1} \frac{\partial G}{\partial y}\left(\frac{\partial \hat{h}}{\partial s}-\gamma \mathrm{i} k \hat{h}\right) \mathrm{d} s+\Lambda\left(\frac{\partial \hat{h}}{\partial y^{2}}-k^{2} \hat{h}\right),
$$

with $\alpha=b_{1} \mathrm{i} k\left\langle\left|u_{0}\right|^{b_{1}} \varphi\right\rangle$. Substituting the cosine series (4.2.20) for $\hat{h}$ results in

$$
\int_{0}^{1} \frac{\partial G}{\partial y} \frac{\partial \hat{h}}{\partial s} \mathrm{~d} s=\sum_{n=1}^{\infty} P_{n}^{1} \tilde{h}_{n} \text { and } \int_{0}^{1} \frac{\partial G}{\partial y} \hat{h} \mathrm{~d} s=\sum_{n=1}^{\infty} P_{n}^{2} \tilde{h}_{n}
$$

with

$$
\begin{aligned}
P_{n}^{1} & =\frac{(n \pi)^{2} \cos (n \pi y)}{(n \pi)^{2}+k^{2}} \\
P_{n}^{2} & =\frac{k\left((-1)^{n}-\cos (k)\right) \frac{\cosh (k y)}{\sinh (k)}+k \sinh (k y)+n \pi \sin (n \pi y)}{(n \pi)^{2}+k^{2}} .
\end{aligned}
$$


The inner products of $P_{n}^{1}$ and $P_{n}^{2}$ with $2 \cos (m \pi y)$ read

$$
\begin{aligned}
& \int_{0}^{1} 2 \cos (m \pi y) P_{n}^{1} \mathrm{~d} y= \begin{cases}\frac{(m \pi)^{2}}{(m \pi)^{2}+k^{2}} & \text { if } m=n, \\
0 & \text { otherwise }\end{cases} \\
& \int_{0}^{1} 2 \cos (m \pi y) P_{n}^{2} \mathrm{~d} y= \begin{cases}0 & \text { if } m+n \text { is even } \\
\frac{-4 m^{2}}{\left(m^{2}-n^{2}\right)\left((m \pi)^{2}+k^{2}\right)} & \text { otherwise }\end{cases}
\end{aligned}
$$

Therefore, substituting the cosine series in the bed evolution equation and taking the inner product with $2 \cos (m \pi y)$ yields

$$
\frac{\partial \tilde{h}_{m}}{\partial \tau}=\omega_{m}^{0} \tilde{h}_{m}+\gamma \sum_{n=1}^{\infty} a_{m n} \tilde{h}_{n}
$$

with $\omega_{m}^{0}$ and $a_{m n}$ as in (4.2.22)-(4.2.23). Truncating the sums at $N$ results in equation (4.2.21). 
5

\section{Local nonlinear tidal bar dynamics}




\begin{abstract}
The long-term dynamics of tidal bars in a confined tidal channel is studied by a new numerical model of intermediate complexity. The simplicity of the model allows a comparison of its results with those obtained from a linear stability analysis of a flat bottom. The aim of this chapter is to study the sensitivity of the tidal bar evolution and possible equilibria to channel width and the Coriolis parameter. For this a configuration that crudely mimics the Exe estuary is chosen. The model simulates tidal bars that extend beyond the middle axis of the channel, as is also observed in nature and in a laboratory. The simulations suggest that while the Coriolis effect strongly alters the initial growth of tidal bars, its impact on the equilibrium patterns is smaller. In one simulation (in which the channel is twice as wide as in the default setting) the bars seem to have reached a morphodynamic equilibrium after approximately a century, but changed to a drastically different pattern after approximately two and a half centuries. The pattern after a century was strongly influenced by the Coriolis effect, however, the pattern after 2.5 centuries was not. This highlights the problem of studying the long-term evolution using time integrations: it is unclear when and if a pattern corresponds to a morphodynamic equilibrium. To overcome this problem, equilibrium patterns and their stability are analysed by means of Newton's method. This results in morphodynamic equilibria for different channel widths. For increasing widths, the bars extend further over the channel axes and the maximum bottom height of the patterns moves away from the channel boundary.
\end{abstract}




\subsection{Introduction}

In the preceding chapters, only the initial formation of tidal bars was studied. In that stage of their formation the heights of the bars are assumed to be small compared to the water depth. To gain insight in the evolution of bars after this initial stage, various approaches can be taken. One such approach employs high-complexity numerical models. In this approach, typically an entire channel from the sea (an open boundary) up to the landward boundary (closed or river) is considered. Usually, an initially sloping bottom topography is imposed and a tidal wave enters the channel from the open boundary. This approach is followed in, for example, Hibma et al. (2003, 2004), van der Wegen and Roelvink (2008), Tambroni et al. (2010), Xie et al. (2017) and Olabarrieta et al. (2018). However, the complexity of such models hinders a detailed analysis of the dynamics that are responsible for the observed bottom patterns and their sensitivity to model parameters.

The models described in the paragraph above are global models. So-called local models can be employed, typically in an idealized setting, when aiming at gaining a more fundamental understanding about the dynamics of tidal bars. In these models, only a short section of a long channel, with a constant width and depth is considered. The water motion in this domain is driven by a spatially uniform pressure gradient and periodic boundary conditions are imposed. This allows one to study the formation of tidal bars without considering the effects of the open and closed boundaries or the morphodynamic adjustment of the sloping bottom topography. Such local models were successful in unraveling the physical mechanism of the initial growth of tidal bars (Seminara and Tubino, 2001, Schramkowski et al., 2002, see also Chapters 3 and 4).

When tidal bars mature, their heights are no longer small compared to the water depth and their dynamics become nonlinear. Nonlinear dynamics can result in complex phenomena, such as multiple equilibria, limit cycles (i.e., bottom patterns that evolve periodically in time) and/or chaos. Schramkowski et al. (2004) investigated whether such phenomena occur in the dynamics of tidal bars. In contrast to most studies on nonlinear bars, they did this by using a local, mathematically tractable model in which the governing equations were projected on Fourier modes. Next, they considered the possible morphodynamic equilibria and their stability properties by performing a bifurcation analysis in two bifurcation parameters, the bottom friction coefficient and the channel width. The starting point of this analysis was the trivial morphodynamic equilibria that describes a uniform tide over a flat horizontal bottom. They found that, depending on the channel width, the flat bottom equilibrium becomes linearly unstable through a critical or subcritical pitchfork bifurcation when increasing the friction parameter beyond a critical value. The manifestation of a subcritical bifurcation implies the existence of multiple equilibria. Further increasing the friction parameter resulted in a Hopf bifurcation that 
leads to time periodic solutions. However, their model approach was limited to weak friction; it turned out that for (realistically) large friction parameter values, too many (and unclear which) Fourier modes were needed. This made the continuation to realistic values of bottom friction computationally unfeasible.

In this chapter, we build a numerical local model, which is not limited to weak bottom friction. This model allows us to explore the long-term evolution and, if existent, nontrivial morphodynamic equilibria that represent tidal bars, where the search for morphodynamic equilibria is restricted to steady states. The study of Schramkowski et al. (2004) motivates the first question that we aim to answer in this chapter: 1) In the new model, do nontrivial tidal bar equilibria exist in the parameter regime with realistic values of the friction parameter? The second question concerns the sensitivity of the longterm dynamics to model parameters. Field observations of Dalrymple and Rhodes (1995) and Leuven et al. (2016) suggest that the channel width is an important parameter for the tidal bar dynamics. This finding is also observed in the available local models and complex numerical models (see earlier citations). Moreover, numerical studies (Xie et al., 2017, Olabarrieta et al., 2018) and the results of Chapter 4 show that there are physically relevant parameter regimes (for moderate to small values of the Rossby number) where the Coriolis force significantly affects the initial growth of the spatial patterns of tidal bars. This motivates the second question: 2) How does the long-term evolution and possibly equilibria patterns depend on channel width and the Coriolis parameter? Lastly, in the linear stability analyses of Seminara and Tubino (2001) and Schramkowski et al. (2002) (see also Chapters 3 and 4) the fastest growing bottom perturbation of a flat bottom was studied, because this pattern dominates the spatial structure of the pattern that initially forms. This raises the third question: 3) To what extend do bottom patterns on the long-term resemble the fastest growing patterns found with the linear stability analysis of the flat bottom?

This chapter is organized as follows. In Section 5.2, the model and the numerical implementation is described. This section is followed by a description of the numerical experiments and their results. It starts with verifying that the model reproduces results of Chapter 3. Subsequently, the output of four time integration simulations of the long-term evolution with different channel width and latitude values is presented. The results of the time integration runs are followed by the (preliminary) results obtained using a numerical continuation approach. Here, the sensitivity of the morphodynamic equilibria to channel width is systematically investigated. In Section 5.4, a comparison of the model results with observed tidal bars is discussed together with the extent to which the long-term evolution and possibly equilibria patterns resemble the fastest growing patterns found with the linear stability analysis of the flat bottom. Section 5.4 ends with discussing limitations of the model and it is followed by the conclusions in Section 5.5. 

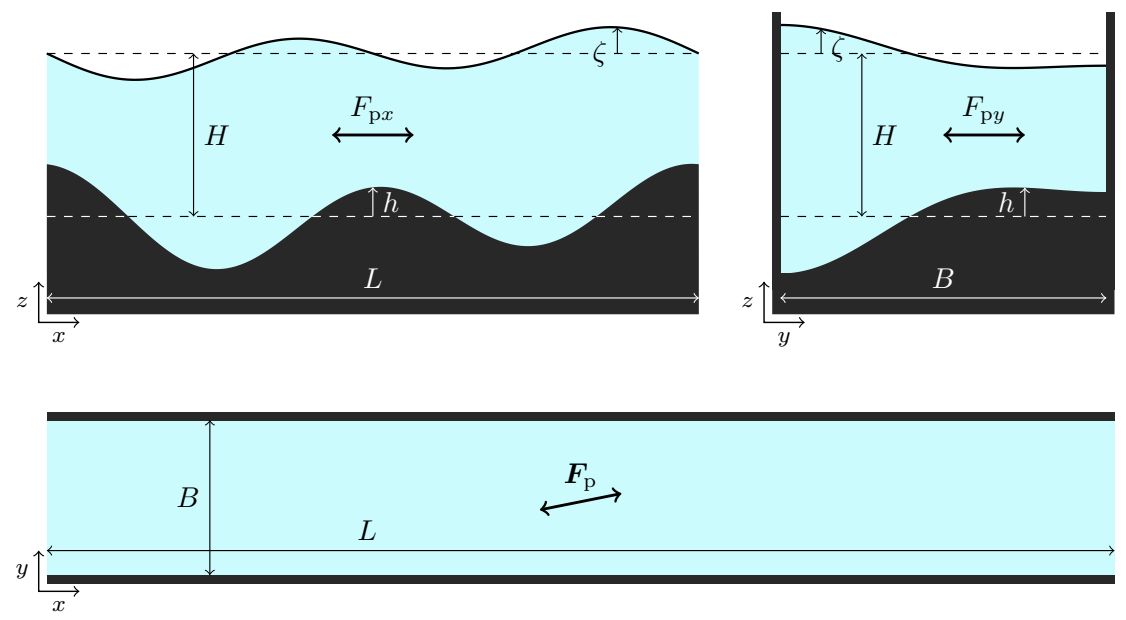

Figure 5.1: Model domain viewed from an along-channel cross-section (top-left panel), a lateral cross-section (top-right panel) and from the top (bottom panel). Here, $x, y$ and $z$ are the along-channel, lateral and vertical coordinate, $L$ denotes the length, $B$ the width and $H$ the undisturbed channel depth. The bottom height is denoted by $h$, the sea surface elevation by $\zeta$. Lastly, $\boldsymbol{F}_{\mathrm{p}}=\left(F_{\mathrm{p} x}, F_{\mathrm{p} y}\right)$ denotes the imposed tidal pressure gradient force.

\subsection{Methods}

\subsubsection{Model}

The local numerical morphodynamic model is based on the Morfo55 model, described in Yuan et al. (2016) (originally developed by Caballeria et al., 2002, Garnier et al., 2006). Here, we present a new model, adopted from the Morfo55 model, to study the dynamics of bottom patterns in a section of a confined tidal channel. Because the original Morfo55 model was designed to study bottom patterns at the coast or on the continental shelf (in a periodic domain), a second closed boundary condition had to be added to allow for a channel geometry. Furthermore, other formulations for sediment transport are added for consistency with the previous two chapters.

The model domain consists of a short section of a long channel. The section has length $L$, width $B$ and undisturbed water depth $H$ (see Figure 5.1). The the domain length is assumed to be short compared to the tidal wavelength and the length scale on which the channel width varies. Moreover, the shallow water approximation is used, the tidal amplitude is assumed to be small compared to the undisturbed depth and the bottom is assumed to evolve on a timescale much longer than the tidal period. Finally, the sediment is assumed to be uniform and the water motion is forced by an imposed spatially uniform tidal 
pressure gradient force.

Let $\boldsymbol{x}=(x, y)$ denote the spatial coordinate, with $x$ the along-channel coordinate and $y$ the lateral coordinate of the domain $[0, L] \times[0, B]$ and let $t$ denote the time coordinate. The evolution of the bottom height $h(t, \boldsymbol{x})$, with respect to the mean bed level $z=-H$, is governed by

$$
\frac{\partial h}{\partial t}=-\frac{1}{1-p} \nabla \cdot\left\langle\boldsymbol{q}_{\mathrm{b}}+\boldsymbol{q}_{\mathrm{s}}\right\rangle .
$$

Here, $\boldsymbol{\nabla}=(\partial / \partial x, \partial / \partial y), p$ is the porosity parameter and $\langle\cdot\rangle$ denotes the average over one tidal period $2 \pi / \sigma$ with $\sigma$ the principal frequency of the imposed tidal pressure gradient force $\mathbf{F}_{\mathrm{p}}$. The right-hand side of the bottom evolution equation (5.2.1) contains the tidally-averaged convergence of the bedload sediment transport $\boldsymbol{q}_{\mathrm{b}}=\left(q_{\mathrm{b} x}, q_{\mathrm{b} y}\right)$ and the suspended load sediment transport $\boldsymbol{q}_{\mathrm{s}}=\left(q_{\mathrm{s} x}, q_{\mathrm{s} y}\right)$.

The formulation of the bedload sediment transport is based on the formulation from Bagnold (1963) with a bed slope term adopted from Bailard (1981), and reads

$$
\boldsymbol{q}_{\mathrm{b}}=\alpha_{\mathrm{b}} \mathcal{H}\left(u_{\mathrm{e}}^{2}-u_{\mathrm{c}}^{2}\right)\left(u_{\mathrm{e}}^{2}-u_{c}^{2}\right)\left(\boldsymbol{u}-\Lambda u_{\mathrm{e}} \nabla h\right),
$$

where $\alpha_{\mathrm{b}}$ is a bed load constant, $\mathcal{H}$ the Heaviside function (which is one when its argument is positive and zero otherwise), $u_{\mathrm{c}}$ the critical velocity for erosion and $\Lambda$ is a bed slope constant. Furthermore, $u_{e}$ is the effective velocity given by (Roos et al., 2004),

$$
u_{e}^{2}=\|\boldsymbol{u}\|^{2}+\frac{1}{2}\left(\frac{u_{w} H}{H+\zeta-h}\right)^{2},
$$

with $\boldsymbol{u}(t, \boldsymbol{x})=(u, v)$ the depth-averaged current velocity, $u_{w}$ the near-bed wave orbital velocity amplitude (taken constant here) and $\zeta(t, \boldsymbol{x})$ the free surface elevation induced by the bottom perturbations. The second term crudely accounts for the effect of wave stirring on sediment transport.

The suspended load sediment transport and concentration is calculated following (the appendix of) ter Brake and Schuttelaars (2010). The suspended load transport reads

$$
\boldsymbol{q}_{\mathrm{s}}=\boldsymbol{u} C-\mu\left(\boldsymbol{\nabla} C+c_{\mathrm{b}} \boldsymbol{\nabla} h+c_{\mathrm{t}} \boldsymbol{\nabla} \zeta\right) .
$$

Here, $C(t, \boldsymbol{x})$ is the depth-integrated volumetric sediment concentration. The last three terms in equation (5.2.3) represent horizontal diffusion of sediment and result from assuming an approximate balance between the vertical turbulent mixing and downward settling which fixes the vertical distribution of sediment concentration. Furthermore, $\mu$ is the horizontal eddy diffusivity (taken constant here) and $c_{\mathrm{b}}$ and $c_{\mathrm{t}}$ are the sediment concentration at the surface and at the bottom, respectively. The latter two are given by

$$
c_{b}=\frac{w_{\mathrm{s}} C}{\kappa_{v}}\left(1-e^{-\frac{w_{\mathrm{s}}}{\kappa_{v}}(H+\zeta-h)}\right)^{-1} \quad \text { and } \quad c_{t}=\frac{w_{\mathrm{s}} C}{\kappa_{v}}\left(e^{\frac{w_{\mathrm{s}}}{\kappa_{v}}(H+\zeta-h)}-1\right)^{-1},
$$


with $w_{\mathrm{s}}$ the settling speed and $\kappa_{v}$ the vertical eddy diffusivity (also taken constant). The concentration $C$ is calculated using an advection-diffusion equation,

$$
\frac{\partial C}{\partial t}+\nabla \cdot \boldsymbol{q}_{\mathrm{s}}=\alpha_{\mathrm{s}} \mathcal{H}\left(u_{\mathrm{e}}^{2}-u_{\mathrm{c}}^{2}\right)\left(u_{\mathrm{e}}^{2}-u_{\mathrm{c}}^{2}\right)-w_{\mathrm{s}} c_{\mathrm{b}},
$$

where the first term on the right hand side models the erosion of sediment from the bed, with $\alpha_{\mathrm{s}}$ an erosion parameter. The second term models the deposition of suspended sediment.

To obtain the sediment transport, the tidal current $\boldsymbol{u}$ and sea surface elevation $\zeta$ are needed. These are calculated by solving the depth-averaged shallow water equations, which consist of the continuity and momentum equations,

$$
\begin{aligned}
\frac{\partial \zeta}{\partial t}+\boldsymbol{\nabla} \cdot((H+\zeta-h) \boldsymbol{u}) & =0, \\
\frac{\partial \boldsymbol{u}}{\partial t}+(\boldsymbol{u} \cdot \boldsymbol{\nabla}) \boldsymbol{u}+\frac{c_{\mathrm{d}}\|\boldsymbol{u}\| \boldsymbol{u}}{H+\zeta-h}+g \boldsymbol{\nabla} \zeta+f \mathbf{E} \boldsymbol{u} & =\boldsymbol{F}_{\mathrm{p}} .
\end{aligned}
$$

Here, $c_{\mathrm{d}}$ is a drag coefficient, $g$ the gravitational acceleration, $\boldsymbol{F}_{\mathrm{p}}(t)=\left(F_{\mathrm{p} x}, F_{\mathrm{p} y}\right)$ an externally prescribed pressure gradient force that drives the tidal flow and $f=2 \Omega \sin (\varphi)$ the Coriolis parameter, where $\Omega$ the rotation rate of the Earth and $\varphi$ the latitude (both taken as constant within the domain). The matrix $\mathbf{E}$ reads

$$
\mathbf{E}=\left(\begin{array}{cc}
0 & -1 \\
1 & 0
\end{array}\right)
$$

Following Yuan et al. (2016), the external pressure gradient force per unit mass is given by

$$
\boldsymbol{F}_{\mathrm{p}}=\frac{\partial \boldsymbol{u}_{0}}{\partial t}+\frac{c_{\mathrm{d}}\left\|\boldsymbol{u}_{0}\right\| \boldsymbol{u}_{0}}{H}+f \mathbf{E} \boldsymbol{u}_{0},
$$

with $\boldsymbol{u}_{0}=(U \sin (\sigma t), 0)$ the current with amplitude $U$ and frequency $\sigma$ induced by the external pressure gradient in the absence of bottom patterns. That is, $\boldsymbol{F}_{\mathrm{p}}$ is chosen such that when $h=0$ the current $\boldsymbol{u}(t, \boldsymbol{x})=\boldsymbol{u}_{0}(t)$.

To solve the system of equations (5.2.1)-(5.2.6), periodic boundary conditions are imposed in the along-channel direction with period $L$ for all variables $h, \zeta, \boldsymbol{u}$ and $C$. Furthermore, there is no transport of water and sediment through the channel walls, resulting in the lateral boundary conditions

$$
v=0 \quad \text { and } \quad q_{\mathrm{s} y}=q_{\mathrm{b} y}=0 \quad \text { at } \quad y=0, B .
$$

\subsubsection{Numerical implementation}

While Morfo55 was implemented in Fortran, the current model is implemented in the Julia programming language (Bezanson et al., 2017). As a result, compared to the Fortran code, the model is easier to read, less prone to errors and it integrates well with existing scientific computing Julia software. Following the 


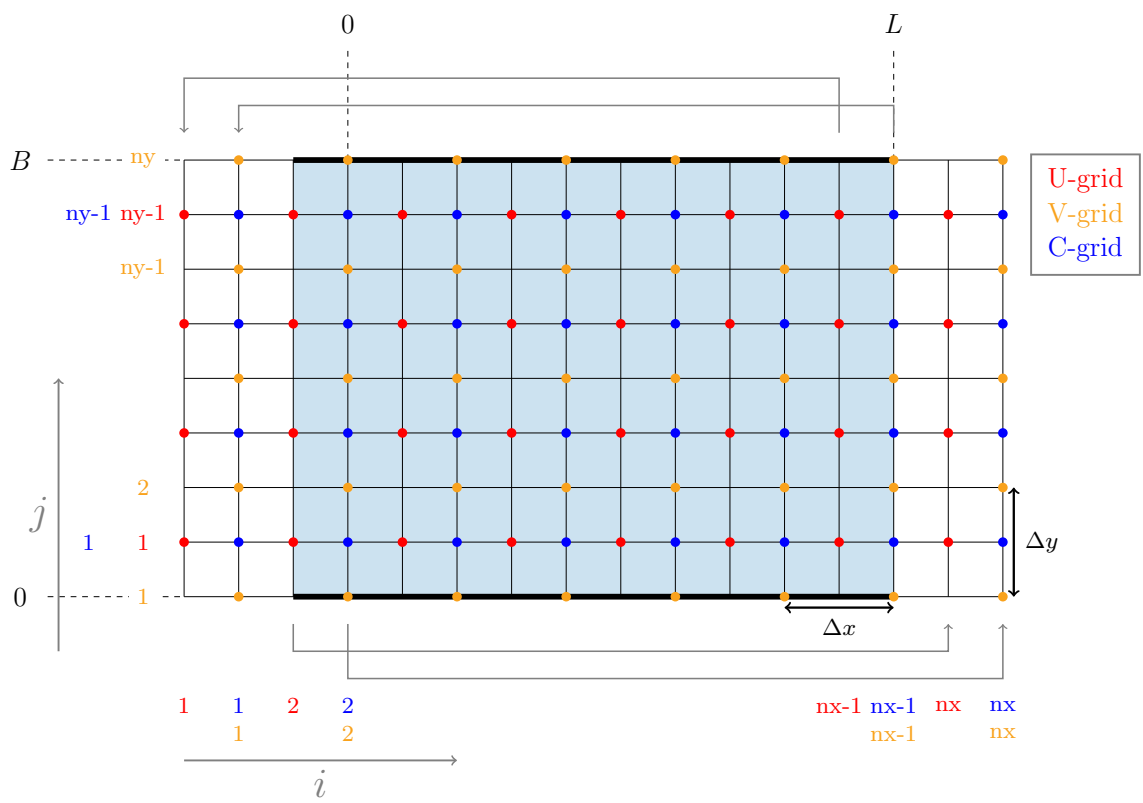

Figure 5.2: Top view of the discretized model domain. The shaded blue area denotes the physical domain. The points outside the shaded area are the ghost points to implement the periodic boundary condition. To that end, the values at the end and beginning of the physical domain are copied to the ghost points (represented by the gray arrows). 
Morfo55 model, the equations are discretized in space by second-order finite difference methods on a regular, rectangular staggered Arakawa C-grid with resolution $\Delta x$ by $\Delta y$ in the along-channel and lateral direction, respectively. This grid consists of three subgrids, the $U-, V$ - and $C$-grid, which are shifted with respect to each other (see Figure 5.2). The along-channel current $u$ and the sediment transports $q_{\mathrm{s} x}$ and $q_{\mathrm{b} x}$, are calculated at the $U$-grid. The lateral current $v$ and sediment transports $q_{\mathrm{s} y}$ and $q_{\mathrm{b} y}$ are calculated at the $V$-grid. The concentration $C$, the free surface elevation $\zeta$ and the bottom height $h$ are calculated at the $C$-grid. The $U$ - and $C$-grid have $N=\left(n_{x}-2\right)\left(n_{y}-1\right)$ grid points in the physical domain (denoted with shaded blue in the figure), while the $V$-grid contains $N_{v}=\left(n_{x}-2\right) n_{y}$ grid points.

Apart from the grid points in the physical domain, so-called ghost points are defined. These ghost points are used to implement the periodic boundary conditions (in the $x$-direction). That is, the values at $\left(x_{2}, y_{j}\right)$ are copied to $\left(x_{n_{x}}, y_{j}\right)$ for all $j$, as well as the values at $\left(x_{n x-1}, y_{j}\right)$ to $\left(x_{1}, y_{j}\right)$ (depicted in Figure 5.2 by the gray arrows). The lateral boundaries are imposed at the $V$-grid (i.e., the grid where the lateral velocity $v$ and the lateral sediment transports $q_{\mathrm{s} y}$ and $q_{\mathrm{b} y}$ are calculated) by requiring: $v=0, q_{\mathrm{s} y}=0$ and $q_{\mathrm{b} y}=0$.

In the bed evolution equation (5.2.1) the convergence of sediment transport is averaged over one tidal cycle (see Chapter 3 or 4 for a discussion). This separates the long morphodynamical timescale from the short hydrodynamical timescale and allows us to calculate the convergence of sediment transport during one tidal cycle in which the bottom height is held fixed. Let $\boldsymbol{\Psi}_{\boldsymbol{h}}(t)$ be a $\left(3 N+N_{v}\right)$-dimensional vector consisting of the free surface elevation $\zeta$, along-channel current velocity $u$, lateral velocity $v$ and the suspended sediment concentration $C$ at time $t$ at every grid point $\left(x_{i}, y_{j}\right)$ in the physical domain. The subscript of $\boldsymbol{\Psi}_{\boldsymbol{h}}$ refers to an $N$-dimensional vector $\boldsymbol{h}$ containing the bottom height $h$ at every grid point $\left(x_{i}, y_{j}\right)$ in the physical domain. It denotes that $\boldsymbol{\Psi}_{\boldsymbol{h}}$ is calculated given fixed values of the elements of $\boldsymbol{h}$. The discretized hydrodynamic equations (5.2.5)-(5.2.6) and the concentration equation (5.2.4), read

$$
\frac{\partial \boldsymbol{\Psi}_{\boldsymbol{h}}}{\partial t}=\boldsymbol{g}_{\boldsymbol{h}}\left(\boldsymbol{\Psi}_{\boldsymbol{h}}\right),
$$

with $\boldsymbol{g}_{\boldsymbol{h}}: \mathbb{R}^{3 N+N_{v}} \rightarrow \mathbb{R}^{3 N+N_{v}}$ a nonlinear function, where the subscript again denotes the fixed values of the bottom height. Solving equation (5.2.8) can be thought of as writing $\zeta, \boldsymbol{u}$ and $C$ in terms of $h$, which allows us to write the the full system of discretized equations as one system of ordinary differential equations (ODEs),

$$
\frac{\partial \boldsymbol{h}}{\partial t}=\boldsymbol{f}(\boldsymbol{h}),
$$

with $\boldsymbol{f}: \mathbb{R}^{N} \rightarrow \mathbb{R}^{N}$ a nonlinear function representing the convergence of tidally averaged sediment transport at every grid point. 


\subsubsection{Time integration}

To solve $\boldsymbol{\Psi}_{\boldsymbol{h}}(t)$ for a fixed bottom height $\boldsymbol{h}$, the ODEs in (5.2.8) are integrated over one tidal cycle with the Runge-Kutta 4 method (RK4) with fixed time steps of $\Delta t$ (Shampine, 2005, Rackauckas and Nie, 2017). Knowing $\boldsymbol{\Psi}_{\boldsymbol{h}}(t)$ over a complete tidal cycle allows the calculation of the convergence of the tidally averaged sediment transport $\boldsymbol{\nabla} \cdot\left\langle\boldsymbol{q}_{\mathrm{s}}+\boldsymbol{q}_{\mathrm{b}}\right\rangle$, resulting in $\boldsymbol{f}(\boldsymbol{h})$. After a certain number of spin-up cycles of equation (5.2.8) (for realistic values of model parameters, this is typically 2), equation (5.2.9) is subsequently integrated in time by the Euler Forward method with time steps of $\Delta \tau$.

\subsubsection{Continuation}

To systematically investigate the dependency of morphodynamic equilibria and their stability to model parameters, such as channel width and latitude, a predictor-corrector continuation technique is used (e.g., Kuznetsov, 2004). Given a parameter setting $P$ and a morphodynamic equilibrium $\boldsymbol{h}$, the predictor predicts a new equilibrium $\boldsymbol{h}^{\prime}$ for the parameter setting $P^{\prime}$ in which the parameters are slightly changed. That is, it transforms the equilibrium $\boldsymbol{h}$, of parameter setting $P$ to a pattern $\boldsymbol{h}^{\prime}$ for which $\boldsymbol{f}\left(P^{\prime}, \boldsymbol{h}\right)$ is close to zero. The corrector, in turn, corrects this pattern such that it is in fact an equilibrium and satisfies

$$
\boldsymbol{f}\left(P^{\prime}, \boldsymbol{h}^{\prime}\right)=0 .
$$

Using the continuation methodology, equation (5.2.9) is replaced by equation (5.2.10). Hence, only the time integration of equation (5.2.8) is still necessary. When the channel width is varied, not only a parameter, but also the grid changes. The predictor in that case consists of stretching or squeezing the previous solution by $\Delta y$, followed by an interpolation onto the numerical $C$-grid. The corrector step is done with the Newton method implemented in BifurcationKit.jl (Veltz, 2020).

The boundary conditions are such that the total amount of sediment is not allowed to change. When using Newton's method, this restriction is not necessarily satisfied. Moreover, the phase of the bottom pattern is not uniquely determined: if $h(x, y)$ is an equilibrium, also $h(x+c, y)$, with $c$ a constant real number, will result in a zero right hand side of equation (5.2.1) since the problem is translational invariant. The nonuniqueness of solutions to equation (5.2.10) hinders the Newton method. To remedy this, $f$ is adapted in two ways. Denote the adapted $\boldsymbol{f}$ with a tilde: $\tilde{\boldsymbol{f}}$. The $i$-th component of $\boldsymbol{h}, \boldsymbol{f}$ and $\tilde{\boldsymbol{f}}$ are denoted by $\boldsymbol{h}_{i}, \boldsymbol{f}_{i}$ and $\tilde{\boldsymbol{f}}_{i}$, respectively for $i=1, \ldots, N$. For $i=2, \ldots, N-1$, the components of $\boldsymbol{f}$ and $\tilde{\boldsymbol{f}}$ are the same, $\boldsymbol{f}_{i}=\tilde{\boldsymbol{f}}_{i}$. The first adaptation of $\boldsymbol{f}$ is that its first component is replaced by a Dirichlet condition that determines the phase of the pattern: $\tilde{\boldsymbol{f}}_{1}(\boldsymbol{h})=\boldsymbol{h}_{1}$. This results in equilibria where $\boldsymbol{h}_{1}=h(0,0)=0$. The second adaptation assures mass conservation 
by imposing a zero mean bottom height: $\tilde{\boldsymbol{f}}_{N}(\boldsymbol{h})=\sum_{i} \boldsymbol{h}_{i}$. That is, the last equation in (5.2.10) is replaced by an integral condition.

To find equilibria with the Newton method, the Jacobian matrix of $\tilde{\boldsymbol{f}}$ is needed. This matrix, evaluated at a bottom pattern $\boldsymbol{h}$, reads

$$
\tilde{\mathbf{J}}(\boldsymbol{h})=\left(\begin{array}{ccc}
\frac{\partial \tilde{\boldsymbol{f}}_{1}}{\partial \boldsymbol{h}_{1}}(\boldsymbol{h}) & \cdots & \frac{\partial \tilde{\boldsymbol{f}}_{1}}{\partial \boldsymbol{h}_{N}}(\boldsymbol{h}) \\
\vdots & \ddots & \vdots \\
\frac{\partial \tilde{\boldsymbol{f}}_{N}}{\partial \boldsymbol{h}_{1}}(\boldsymbol{h}) & \cdots & \frac{\partial \tilde{\boldsymbol{f}}_{N}}{\partial \boldsymbol{h}_{N}}(\boldsymbol{h})
\end{array}\right) .
$$

Since $\tilde{\boldsymbol{f}}$ contains a time integral, calculating $\tilde{\mathbf{J}}$ by hand is complicated. Options to calculate the Jacobian are the use of symbolic software or by employing finite differences (Dijkstra et al., 2014). Here, we opt for the (forward) automatic differentiation method (see e.g., Griewank and Walther, 2008). This boils down to using the chain rule consistently and smart bookkeeping. The idea is to save both the result and the derivative after every elementary operation of the code that calculates $\tilde{\boldsymbol{f}}(\boldsymbol{h})$ (e.g., addition, multiplication, etc.). When $\tilde{\boldsymbol{f}}$ is differentiable at $\boldsymbol{h}$, this results in the calculation of the Jacobian matrix $\tilde{\mathbf{J}}(\boldsymbol{h})$ without making truncation errors. The automatic differentiation is done with the ForwardDiff.jl package (Revels et al., 2016).

The stability of an equilibrium $\boldsymbol{h}$ follows from the spectral decomposition of the Jacobian matrix $\mathbf{J}(\boldsymbol{h})$ (no tilde). This matrix is calculated in the same way as $\tilde{\mathbf{J}}(\boldsymbol{h})$ but by taking the derivative of $\boldsymbol{f}$ rather than $\tilde{\boldsymbol{f}}$. That is, without the integral and Dirichlet condition. The eigenvalue problem to be solved reads

$$
\mathbf{J}(\boldsymbol{h}) \boldsymbol{v}=\omega \boldsymbol{v}
$$

with $\boldsymbol{v} \neq 0$ the normalized eigenvectors and $\omega$ the corresponding eigenvalues. The eigenvalues are sorted by their real part: $\operatorname{Re}\left\{\omega_{1}\right\} \geq \cdots \geq \operatorname{Re}\left\{\omega_{N}\right\}$. If the real part of $\omega_{1}$ is negative, the equilibrium $\boldsymbol{h}$ is linearly stable. The corresponding eigenvector $\boldsymbol{v}_{1}$ is in the direction in which perturbations of the equilibrium dampens the slowest. On the other hand, when there are eigenvalues with positive real part, the equilibrium is linearly unstable. The eigenvector $\boldsymbol{v}_{1}$, corresponding to the eigenvalue with the largest real part $\omega_{1}$, points in the direction in which perturbations grow the fastest.

For $\tilde{\boldsymbol{f}}$ and $\boldsymbol{f}$ to be differentiable with respect to $\boldsymbol{h}$, two modifications to the governing equations are made. The first is that the Heaviside function $\mathcal{H}$ in equation (5.2.4) and (5.2.2) is replaced by a hyperbolic tangent in the continuation code,

$$
\mathcal{H}\left(u_{e}^{2}-u_{c}^{2}\right) \approx \frac{1+\tanh \left(\delta_{1}\left(u_{e}^{2}-u_{c}^{2}\right)\right)}{2}
$$

where $\delta_{1}=10 \mathrm{~s}^{2} \mathrm{~m}^{-2}$ tunes the steepness of the smoothed jump. The second is that $\|\boldsymbol{u}\|$ in the quadratic friction term is replaced by $\left(u^{2}+v^{2}+\delta_{2}\right)^{1 / 2}$ with $\delta_{2}=10^{-5} \mathrm{~m}^{2} \mathrm{~s}^{-2}$. These two modifications are only made for the continuation runs, because especially the first is computationally expensive. 


\subsubsection{Analysis of model output}

In Section 5.3, the results are analysed by calculating the root-mean square height at time $t$, which is a measure of the kinetic energy of the bottom pattern and defined as

$$
h_{\mathrm{rms}}(t)=\frac{\|\boldsymbol{h}(t)\|}{\sqrt{N}} \approx\left(\frac{1}{L B} \int_{0}^{L} \int_{0}^{B} h(t, x, y)^{2} \mathrm{~d} x \mathrm{~d} y\right)^{1 / 2},
$$

where || || denotes the Euclidean norm. Moreover, the patterns are analysed with respect to an appropriate Fourier basis. That is, the bottom height at time $t$ is written as

$$
h(t, x, y)=\sum_{m, n=-\infty}^{\infty} h_{m, n}(t) e^{2 \pi \mathrm{i}\left(\frac{n}{2 B} y+\frac{m}{L} x\right)},
$$

with $h_{m, n}$ a complex number for every integer $m$ and $n$. This allows one to analyse the time evolution of the different Fourier coefficients $h_{m, n}$ in both the initial formation and the long-term evolution of the bottom patterns.

\subsubsection{Design of experiments}

In Section 5.3, three types of model experiments will be performed. The setup of each type is discussed below. We base the channel characteristics on those of the Exe estuary in England. That is, the channel is of the order of $3 \mathrm{~m}$ deep, about $1 \mathrm{~km}$ wide and the tidal currents are in the order of $0.5 \mathrm{~m} \mathrm{~s}^{-1}$. A complete description of the default parameter values is listed in Table 5.1. The sections below contain the differences from the default setting for each type of experiment.

\section{Verification}

We verify that the model reproduces results of Chapter 3 in three different manners. First, the growth rates calculated in Chapter 3 are compared with the eigenvalues of the Jacobian matrix $\mathbf{J}(0)$. Second, the eigenvectors of this Jacobian are compared with the eigenfunctions in Chapter 3. The former are supposed to be finite dimensional (i.e., discretized) representations of the latter. Third, the morphodynamic time integration is tested by projecting the bottom height resulting from a time integration run onto the eigenvector of the Jacobian $\mathbf{J}(0)$ with the largest growth rate. The time series of the amplitude of this pattern is compared with the time series of the exponential growth of the corresponding eigenvalue. The morphodynamic time integration is initialized with a random bottom perturbations between -10 and $10 \mathrm{~cm}$ (with mean zero and satisfying the boundary conditions). 


\begin{tabular}{|c|c|c|c|}
\hline Parameter & Value & Unit & Description \\
\hline$B$ & 1 & $\mathrm{~km}$ & channel width \\
\hline$H$ & 3 & $\mathrm{~m}$ & undisturbed channel depth \\
\hline$L$ & 10 & $\mathrm{~km}$ & domain length \\
\hline$\Delta y$ & 50 & $\mathrm{~m}$ & lateral grid space \\
\hline$\Delta x$ & 100 & $\mathrm{~m}$ & longitudinal grid space \\
\hline$\Delta t$ & 5 & $\mathrm{~s}$ & hydrodynamical time step \\
\hline$\Delta \tau$ & 1 & week & morphological time step \\
\hline$U$ & 0.5 & $\mathrm{~ms}^{-1}$ & background current amplitude \\
\hline$\sigma$ & $2 \pi / 44700$ & $\mathrm{~s}^{-1}$ & $\mathrm{M}_{2}$ tidal frequency \\
\hline$g$ & 9.81 & $\mathrm{~ms}^{-2}$ & gravitational acceleration \\
\hline$c_{d}$ & 0.0025 & & drag coefficient \\
\hline$\Omega$ & $7.29 \cdot 10^{-5}$ & $\mathrm{~s}^{-1}$ & angular speed of Earth rotation \\
\hline$\varphi$ & 50 & $\circ \mathrm{N}$ & latitude \\
\hline$u_{c}$ & 0.3 & $\mathrm{~ms}^{-1}$ & critical erosion velocity \\
\hline$w_{s}$ & 0.013 & $\mathrm{~ms}^{-1}$ & settling velocity \\
\hline$\mu$ & 10 & $\mathrm{~m}^{2} \mathrm{~s}^{-1}$ & horizontal eddy diffusivity \\
\hline$\kappa_{v}$ & 0.01 & $\mathrm{~m}^{2} \mathrm{~s}^{-1}$ & vertical eddy diffusivity \\
\hline$\alpha_{\mathrm{s}}$ & $5 \cdot 10^{-6}$ & $\mathrm{~s} \mathrm{~m}^{-1}$ & erosion suspended sediment parameter \\
\hline$\alpha_{\mathrm{b}}$ & $3 \cdot 10^{-4}$ & $\mathrm{~s} \mathrm{~m}^{-1}$ & bedload parameter \\
\hline$u_{w}$ & 0.25 & $\mathrm{~ms}^{-1}$ & near-bed wave orbital velocity amplitude \\
\hline$\Lambda$ & 2 & & bed slope parameter \\
\hline$p$ & 0.4 & & porosity \\
\hline
\end{tabular}

Table 5.1: Model parameters. Their values are based on the Exe estuary (see Chapter 3 and references therein). The value of the near-bed wave orbital velocity amplitude $u_{w}$ is the same as in Yuan et al. (2016) and Roos et al. (2004). 
To compare the model output of the nonlinear model with that of the linear model of Chapter 3, a number of simplifications are made to the current model. First of all, the Coriolis effect is neglected, the quadratic bottom shear stress replaced by a linear one,

$$
\frac{c_{d}\|\boldsymbol{u}\| \boldsymbol{u}}{H+\zeta-h} \rightarrow \frac{8 c_{d} U \boldsymbol{u}}{3 \pi(H+\zeta-h)}
$$

and only the bed slope (diffusive) part in the bedload transport is considered:

$$
\boldsymbol{q}_{\mathrm{b}}=-\alpha_{b} \Lambda\|\boldsymbol{u}\|^{3} \nabla h
$$

Furthermore, the last term in the suspended sediment transport (5.2.3) is neglected, $c_{t}=0$, and in the expression for $c_{b}$, the depth $H+\zeta-h$ is replaced by the constant undisturbed channel depth $H$. Moreover, the critical current velocity for sediment movement and the near-bed wave orbital velocity are set to zero, $u_{c}=0$ and $u_{w}=0$, the channel depth is $H=5 \mathrm{~m}$, the background current velocity amplitude is $U=1 \mathrm{~m} \mathrm{~s}^{-1}$, the eddy diffusivity $\mu=1 \mathrm{~m}^{2} \mathrm{~s}^{-1}$ and the latitude $\varphi=0^{\circ}$. Note that a difference between the two model formulations remains, namely the sea surface elevation $\zeta$ induced by the bottom topography.

\section{Time integration}

To compare the fastest growing patterns obtained from the linear stability analysis with the long-term evolution and to study the (transient) long-term evolution of tidal bars for different values of the channel width $B$ and the Coriolis parameter, four time integration runs are performed. The channel width is chosen to be equal to $B=1 \mathrm{~km}$ or $B=2 \mathrm{~km}$ and the latitude $\varphi=0^{\circ}$ or $\varphi=50^{\circ} \mathrm{N}$. The bottom height is again initialized with random values between -10 and $10 \mathrm{~cm}$ with mean zero and satisfying the boundary conditions. In contrast to the verification experiments, the full model is used, with parameter values as in Table 5.1. The length $L$ of the domain is $10 \mathrm{~km}$. This value is chosen such that multiple bars in the along-channel direction can form. However, its choice remains somewhat arbitrary and the sensitivity of the model results to this choice will be discussed in Section 5.4.

\section{Continuation}

To systematically investigate the effect of channel width on the spatial pattern of the morphodynamic equilibria, a numerical continuation is performed. To speed up the calculations, a slightly simplified model setting is used for this: $u_{c}=0, u_{w}=0, \varphi=0^{\circ}, c_{\mathrm{t}}=0$ and the depth $H-h+\zeta$ in $c_{\mathrm{b}}$ is replaced by the undisturbed depth $H$. The stability of the equilibria is calculated using the Jacobian matrix $\tilde{\mathbf{J}}$, which was calculated during the Newton iteration. That 


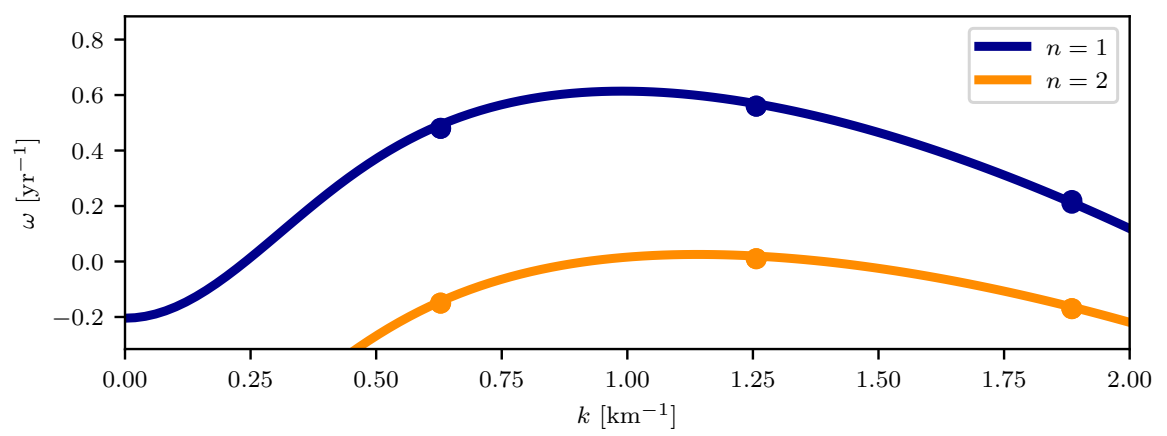

Figure 5.3: Growth rate of perturbations of the trivial equilibrium $h=0$ versus alongchannel wavenumber $k$. The different colors denote different lateral mode numbers. The solid lines are calculated with the model in Chapter 3. The colored dots are the eigenvalues of the Jacobian $\mathbf{J}(0)$ at the trivial equilibrium $\boldsymbol{h}=0$ calculated by the model described in this chapter. The parameters values are as in Table 5.1 with $U=1 \mathrm{~m} / \mathrm{s}, H=5 \mathrm{~m}, u_{c}=0, u_{w}=0, \mu=1 \mathrm{~m}^{2} \mathrm{~s}^{-1}, \varphi=0^{\circ}, c_{\mathrm{t}}=0$, the depth $H-h+\zeta$ in $c_{\mathrm{b}}$ is replaced by the undisturbed depth $H$, the bottom stress is linear in $\boldsymbol{u}$ and the only the bed slope part in the bedload transport is considered.

is, instead of computing $\mathbf{J}$ and solving the eigenvalue problem (5.2.11), the stability is determined by solving the generalized eigenvalue problem $\tilde{\mathbf{J}}(\boldsymbol{h}) \boldsymbol{v}=$ $\omega \mathbf{M} \boldsymbol{v}$, where $\mathbf{M}=\operatorname{diag}(0,1, \ldots, 1,0)$ is a singular diagonal matrix with zeros at the first and last element of the diagonal and ones elsewhere. In the future this could be redone by using $\mathbf{J}$, as described in Section 5.2.4.

\section{$5.3 \quad$ Results}

\subsubsection{Verification}

Using the the model setting discussed in Section 5.2.6, the eigenvalues of the Jacobian are plotted in Figure 5.3, together with the growth rates calculated with the model discussed in Chapter 3 for various along-channel wavenumbers $k$. The figure shows that the correspondence is good. Since the Coriolis effect is neglected, the eigenvectors of $\mathbf{J}(0)$ are discretized versions of

$$
\cos (k(x-\phi))) \cos \left(\frac{n \pi}{B} y\right),
$$

with $k$ and $\phi$ real numbers and $n$ an integer (see Chapter 3). In Figure 5.4 the eigenvectors obtained with the numerical model are plotted. Figure 5.4 shows that that the patterns can be associated with a real number $k$ and integer $n$. Moreover, the presence of two independent eigenvectors per eigenvalue, show that the patterns are translational invariant and that the value of $\phi$ is 


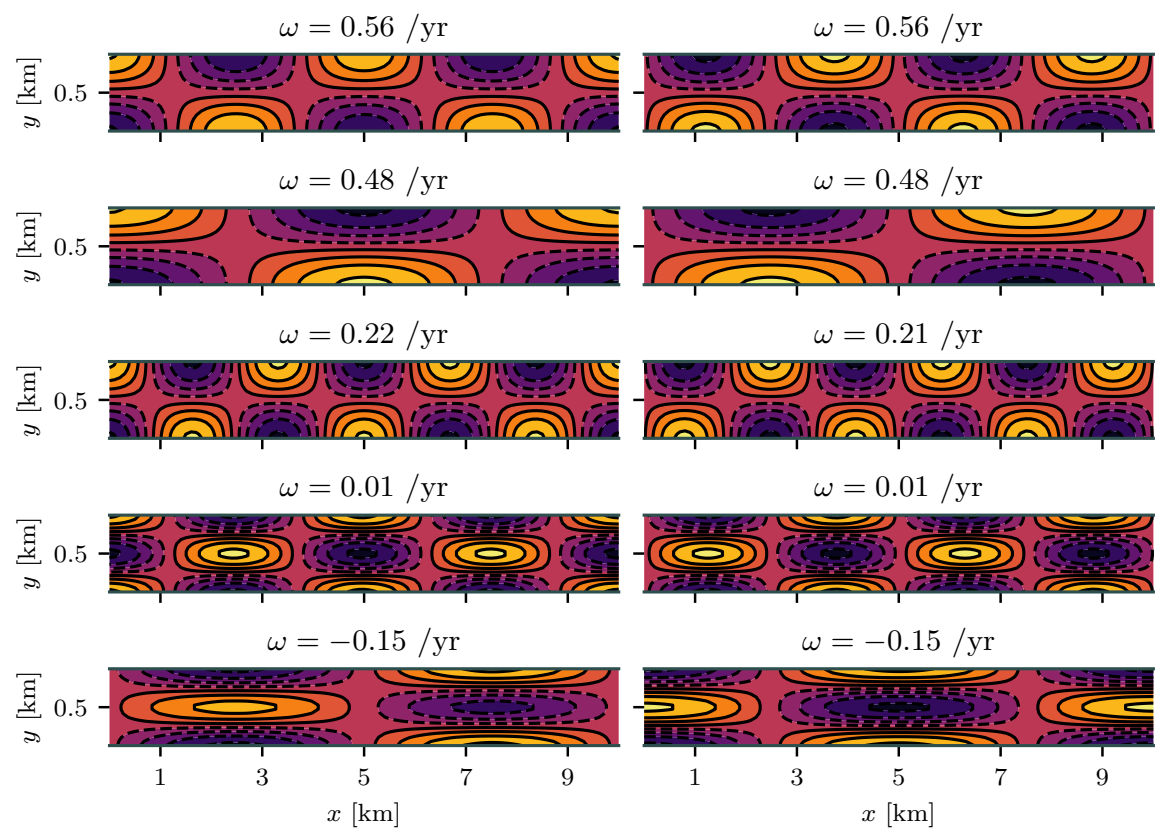

Figure 5.4: Contour-color plots of eigenvectors of the Jacobian $\mathbf{J}(0)$ at the trivial equilibrium $\boldsymbol{h}=0$ corresponding to the largest (in real part) eigenvalues for lateral mode number $n=1$ and $n=2$. The parameters values are as in Table 5.1 with $U=1 \mathrm{~m} / \mathrm{s}, H=5 \mathrm{~m}, u_{c}=0, u_{w}=0, \mu=1 \mathrm{~m}^{2} \mathrm{~s}^{-1}, \varphi=0^{\circ}, c_{\mathrm{t}}=0$, the depth $H-h+\zeta$ in $c_{\mathrm{b}}$ is replaced by the undisturbed depth $H$, the bottom stress is linear in $\boldsymbol{u}$ and the only the bed slope part in the bedload transport is considered. 


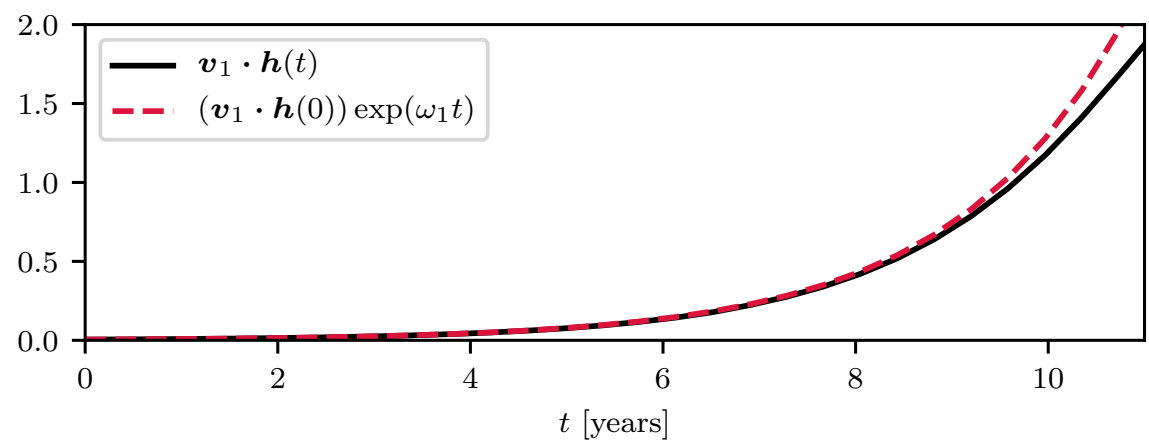

Figure 5.5: The solid black line is the bottom height $\boldsymbol{h}(t)$ projected onto the first eigenvector $\boldsymbol{v}_{1}$ versus time $t: \boldsymbol{v}_{1} \cdot \boldsymbol{h}(t)$ (i.e., the amplitude of the fastest growing bottom pattern versus time). The dashed red line shows the exponential function $\left(\boldsymbol{v}_{1} \cdot \boldsymbol{h}(0)\right) e^{\omega_{1} t}$ (red dashed line), where $\omega_{1}$ is the eigenvalue corresponding to $\boldsymbol{v}_{1}$ The parameters values are as in Table 5.1 with $U=1 \mathrm{~m} / \mathrm{s}, H=5 \mathrm{~m}, u_{c}=0$, $u_{w}=0, \mu=1 \mathrm{~m}^{2} \mathrm{~s}^{-1}, \varphi=0^{\circ}, c_{\mathrm{t}}=0$, the depth $H-h+\zeta$ in $c_{\mathrm{b}}$ is replaced by the undisturbed depth $H$, the bottom stress is linear in $\boldsymbol{u}$ and the only the bed slope part in the bedload transport is considered.

arbitrary. To test the morphodynamic time integration, the flat bottom profile is perturbed with random disturbances and integrated in time. The solid black line in Figure 5.5 shows the time evolution of the bottom height vector $\boldsymbol{h}(t)$, projected onto the first eigenvector (top left panel in Figure 5.4). This evolution compares well with the exponential growth of the eigenvector corresponding to the largest eigenvalue of the Jacobian (dashed red line).

\subsubsection{Time integration}

Figure 5.6 shows the time evolution of the root-mean square height of the four simulations described in Section 5.2.6. In the first two simulations, indicated by the solid black and red lines in the figure, the channel width $B$ is $1 \mathrm{~km}$, while in the third and fourth simulations, indicated by the blue and cyan line, the channel is $2 \mathrm{~km}$ wide. In the first and third simulation the Coriolis parameter is zero (i.e., latitude $\varphi=0^{\circ}$ ), whereas in the second and fourth simulation the latitude is $\varphi=50^{\circ} \mathrm{N}$. After an initial growth, the root-mean square height of the pattern becomes approximately constant in time. This period is longer for the wider channels. The root-mean square height of patterns after 1000 years in the wider channels are larger than those in the narrow channels. Moreover, in the $1 \mathrm{~km}$ wide channels the root-mean square height after 1000 years is slightly larger if the Coriolis effect is included compared to the case where it is not, while the opposite holds for the $2 \mathrm{~km}$ wide channels. 


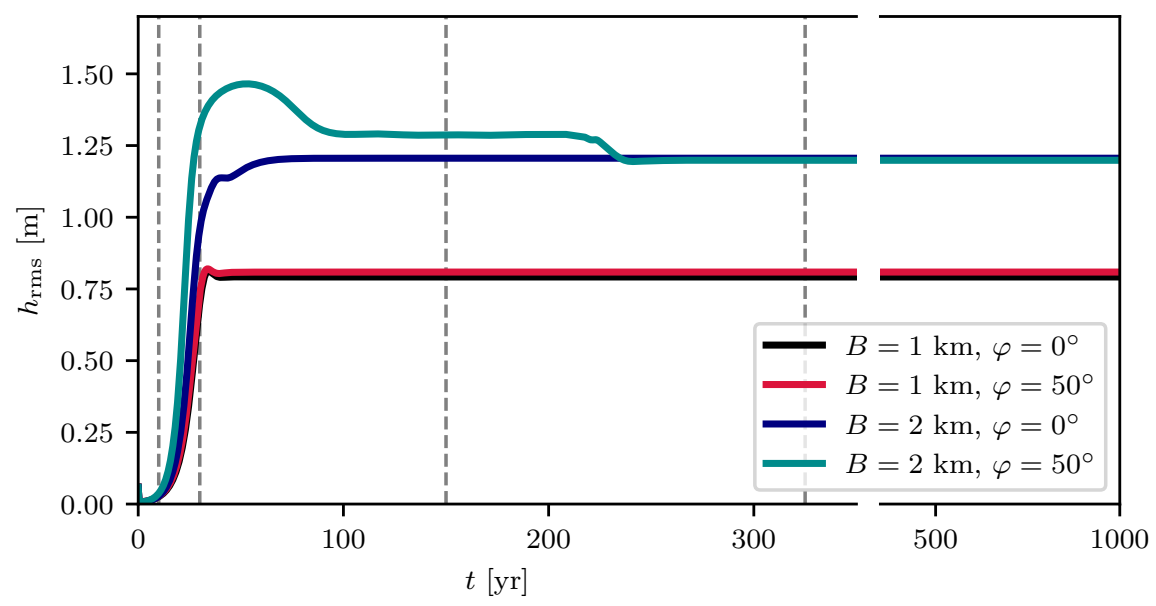

Figure 5.6: Root-mean square height $h_{\mathrm{rms}}$ versus time $t$ for four different simulations. The vertical lines denote the moments in time at which the patterns are shown in Figures 5.7-5.10. Parameter values used are as in Table 5.1. 


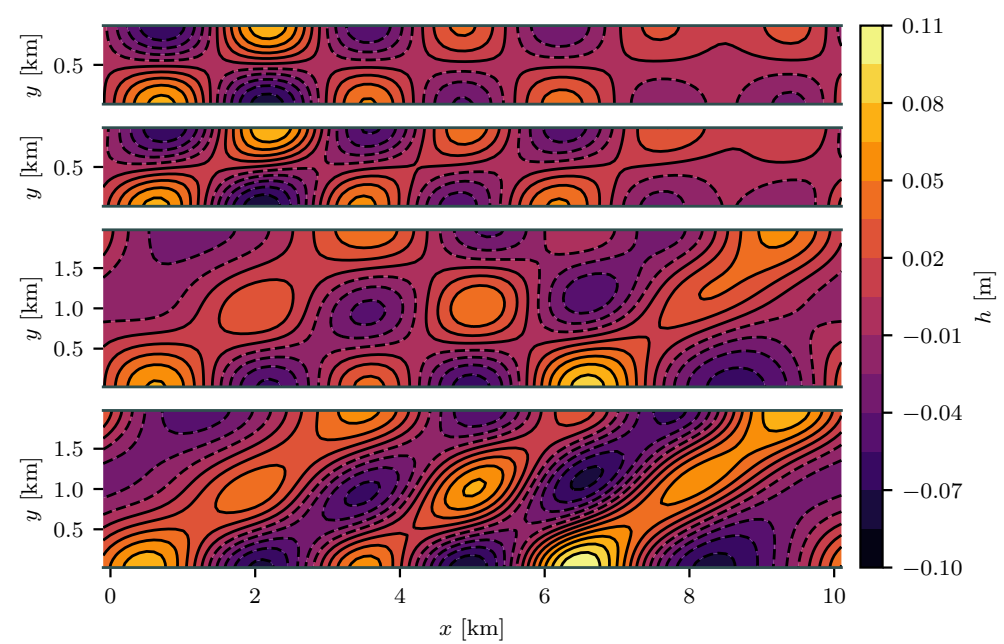

Figure 5.7: Bottom height versus space after $t=10$ years for four different simulations. In the top two panels the channel width $B=1 \mathrm{~km}$ and in the bottom two the channel width is $B=2 \mathrm{~km}$. In the first and third panel the latitude $\varphi=0^{\circ}$, while in the second and bottom panel the latitude $\varphi=50^{\circ}$. Parameters values used are as in Table 5.1.

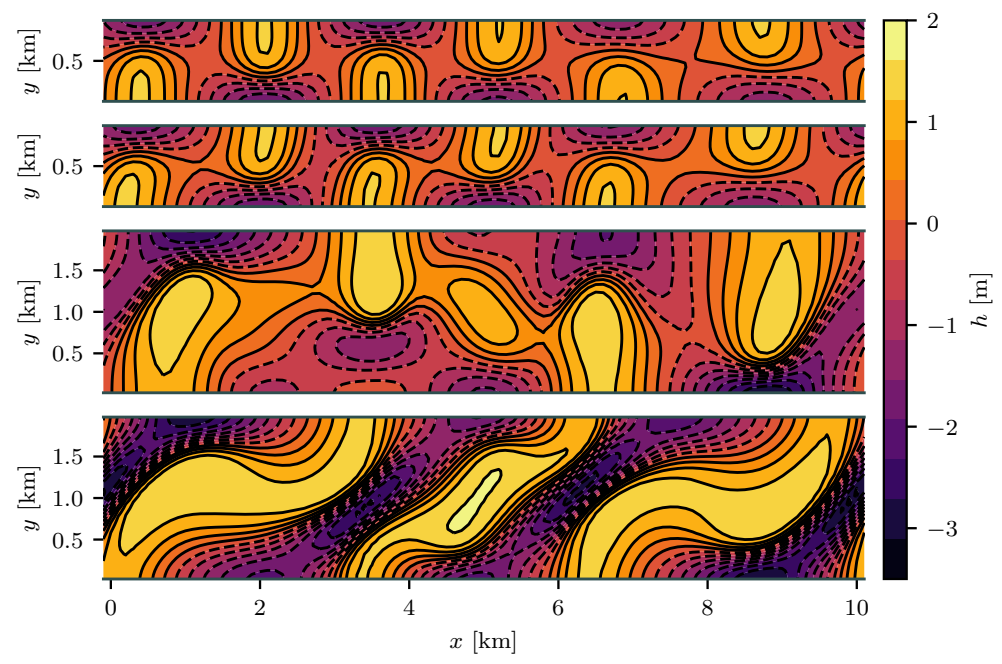

Figure 5.8: As Figure 5.7 but after $t=30$ years. From top to bottom panel: $(B, \varphi)=\left(1 \mathrm{~km}, 0^{\circ}\right),\left(1 \mathrm{~km}, 50^{\circ}\right),\left(2 \mathrm{~km}, 0^{\circ}\right)$ and $\left(2 \mathrm{~km}, 50^{\circ}\right)$. 


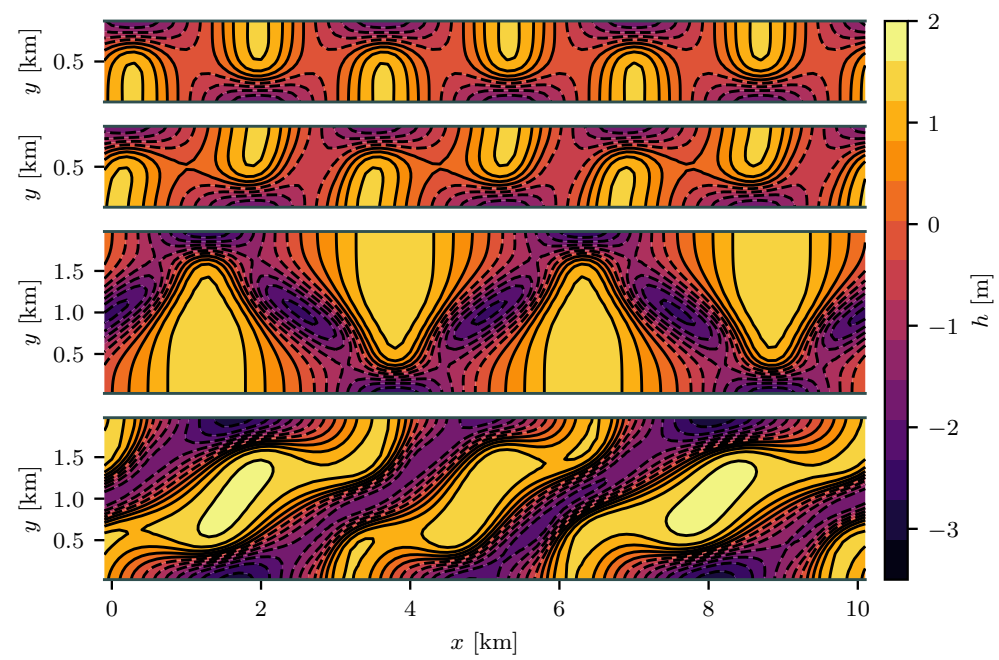

Figure 5.9: As Figure 5.7 but after $t=150$ years. From top to bottom panel: $(B, \varphi)=\left(1 \mathrm{~km}, 0^{\circ}\right),\left(1 \mathrm{~km}, 50^{\circ}\right),\left(2 \mathrm{~km}, 0^{\circ}\right)$ and $\left(2 \mathrm{~km}, 50^{\circ}\right)$.

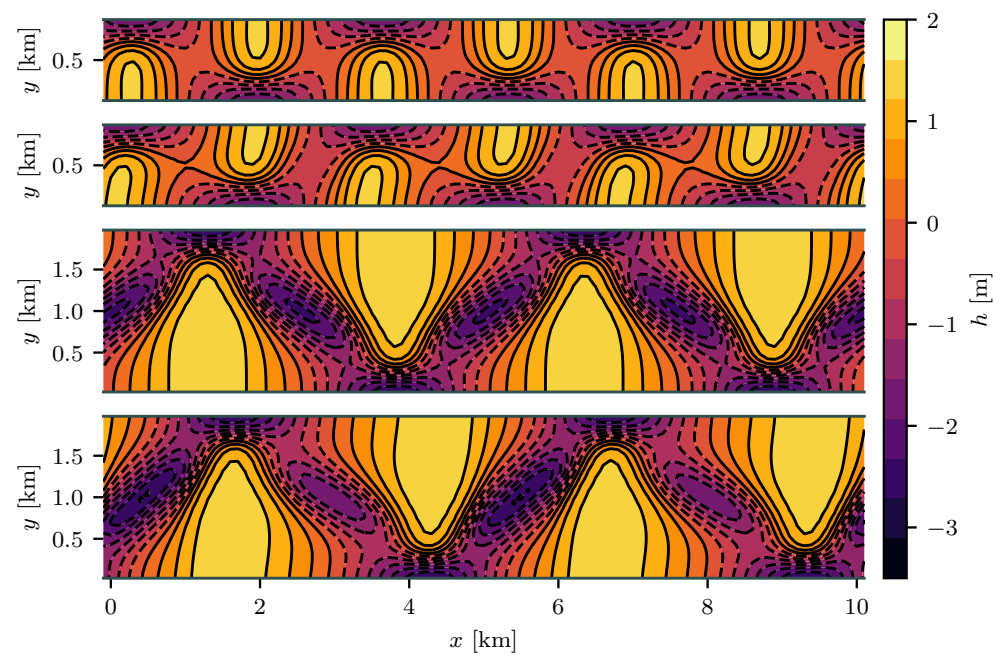

Figure 5.10: As Figure 5.7 but after $t=325$ years. From top to bottom panel: $(B, \varphi)=\left(1 \mathrm{~km}, 0^{\circ}\right),\left(1 \mathrm{~km}, 50^{\circ}\right),\left(2 \mathrm{~km}, 0^{\circ}\right)$ and $\left(2 \mathrm{~km}, 50^{\circ}\right)$. 
Figure 5.7-5.10 show snapshots of the bottom patterns of the four simulations at four different moments in time: $t=10,30,150$ and $325 \mathrm{yr}$. After 10 years (Figure 5.7), the patterns resemble the patterns of the eigenvectors of the Jacobian at the flat bottom corresponding to the largest eigenvalue (see Chapter 4). When the Coriolis effect is neglected $\left(\phi=0^{\circ}\right)$, in the narrow channels $(B=1 \mathrm{~km})$, an alternating bar pattern forms, while in the wider channels ( $B=2 \mathrm{~km})$, a braided pattern with one bar/trough in the middle forms. As suggested by the results of Chapter 4 , in the simulations where the Coriolis effect is included $\left(\phi=50^{\circ} \mathrm{N}\right)$, the pattern is a combination of bars and oblique ridges which are rotated anticlockwise with respect to the main channel axis. The ridges are more pronounced in the wider channel.

After 30 years (Figure 5.8), the narrow channels are close to equilibrium (see Figure 5.6). The wider channels are still in their transient phase. During that phase, the bars in the middle connect to those at the sides. In the simulation with the Coriolis effect included, the bars on the right and left side of the channel are connected, thereby forming meandering ridges.

After 150 years (Figure 5.9), all patterns seem to be stationary (see Figure 5.6). In the first simulations $\left(B=1 \mathrm{~km}, \varphi=0^{\circ}\right)$, a pattern forms with bars extends over the middle axis of the channel. In the second simulation $\left(B=1 \mathrm{~km}, \varphi=50^{\circ} \mathrm{N}\right)$, the pattern is fairly similar to that in the first simulation, but the Coriolis effect skews the pattern slightly. The latter results in the formation of a shallow sill between the alternating bars on one side and a deeper channel on the other side of the bars. In the third simulation $(B=2 \mathrm{~km}$, $\varphi=0^{\circ}$ ), the bars in the middle fully merged with those on the side. Compared to the first simulation, the bars are wider at the closed boundaries and form somewhat triangular bars opposed to the thin finger shaped bars in the narrow channels. In the fourth simulation $\left(B=2 \mathrm{~km}, \varphi=50^{\circ} \mathrm{N}\right)$ the pattern remains in the shape of meandering ridges. They are not completely static, but their evolution is very slow. This period of slow evolution is followed by a quick adjustment after 2.5 centuries (see Figure 5.6). At that time, the ridges break into separate bars and form a pattern similar to the pattern observed in the third simulation in which the Coriolis effect is neglected. The main difference is that in the fourth simulation the pattern is slightly skewed (Figure 5.10).

Figure 5.11 shows the amplitudes of the Fourier coefficients $h_{m, n}$ versus time. The colored lines are the four coefficients with the largest amplitude after 500 years. Their along-channel and lateral wavenumbers are shown in the top panel of the figure. The gray lines are the amplitudes of the remaining coefficients. The figure reveals that in the narrow channel $(B=1 \mathrm{~km})$ the Fourier mode that initially grows the fastest (red line) remains dominant over time. However, in the wider channel this is not the case. Moreover, the bottom panels show the initial difference and eventual similarity between the Fourier spectrum of the $2 \mathrm{~km}$ wide channel with and without the Coriolis effect taken into account. 

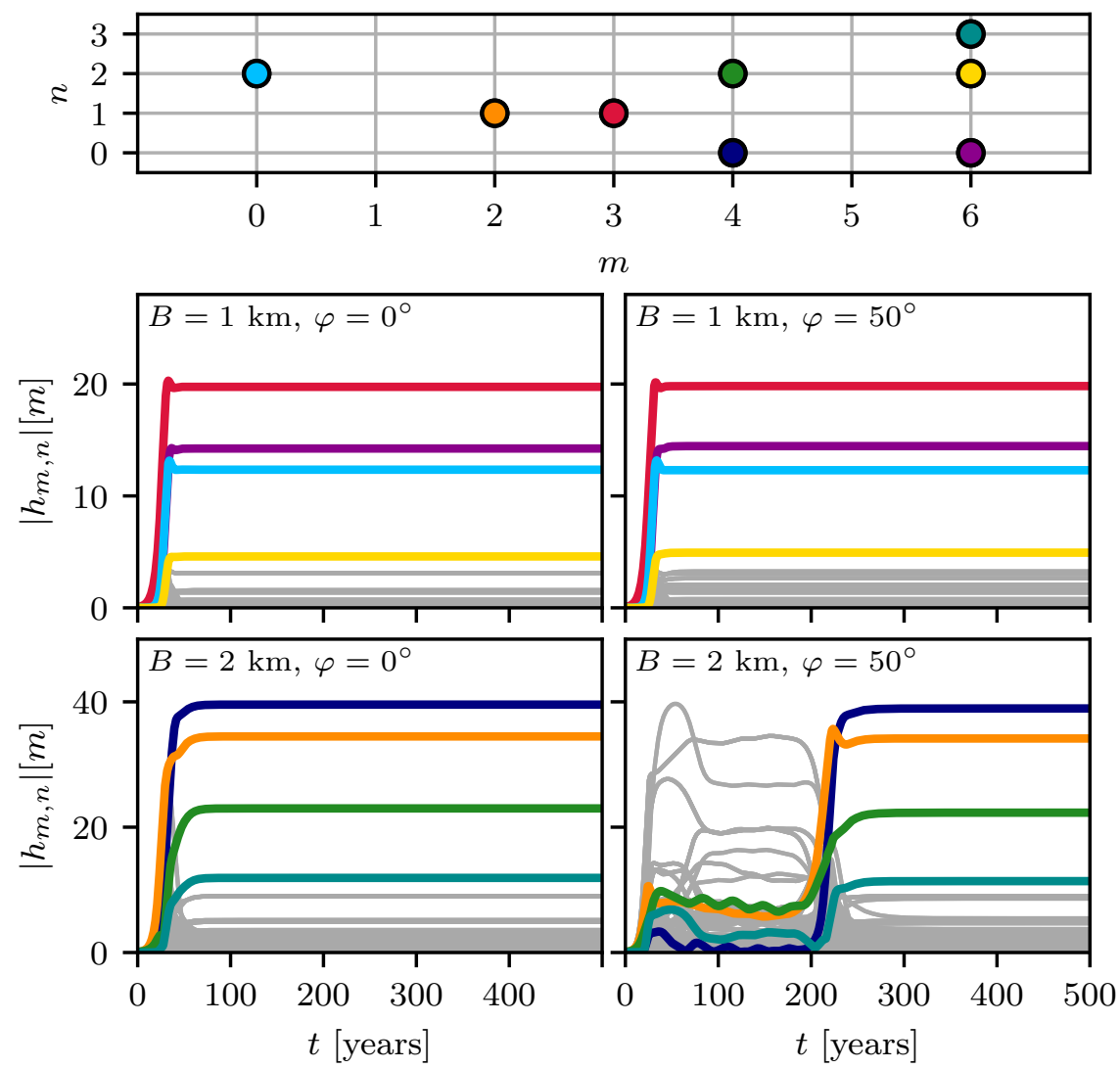

Figure 5.11: Bottom four panels: amplitude of Fourier coefficient $h_{m, n}$ versus time $t$ for the four simulations. The four Fourier modes that have the largest amplitude after 500 years are colored. The corresponding modenumbers $m$ and $n$ are shown in the top panel. Parameter values used are as in Table 5.1. 


\subsubsection{Continuation}

The top panel of Figure 5.12 shows the root-mean square height $h_{\mathrm{rms}}$ of morphodynamic equilibria as a function of channel width $B$. The white dots are unstable equilibria and the gray dots are stable ones. For this parameter setting, the critical channel width at which the flat bottom loses its stability is between $B=0.8$ and $B=0.85 \mathrm{~km}$. The bottom panels of Figure 5.12 show the morphodynamic equilibria for channel widths of $B=0.5,0.85,1,1.5$ and $1.8 \mathrm{~km}$ (indicated by the stars in the top panel of the figure). The first morphodynamic equilibrium is a flat bottom, which is linearly stable for these parameter values. For $B=0.85 \mathrm{~km}$, the equilibrium bathymetry is nontrivial, with the bars slightly extending over the mid-channel axis. For wider channels, the bars are further extended over the channel axes. Moreover, whereas the bars attain their maximum height on the side of the channel for $B=0.85$ and $B=1 \mathrm{~km}$, the location of maximum bottom height is detached from the channel boundary for larger channel widths. The second panel in Figure 5.12 shows the amplitudes of the Fourier coefficients $\left|h_{m, n}\right|(>0.25 \mathrm{~m})$ as a function of channel width $B$. The colors correspond to those in Figure 5.11. The four Fourier modes with the largest amplitude after 500 years in the first time integration run $\left(B=1 \mathrm{~km}\right.$ and $\varphi=0^{\circ}$, see Figure 5.11) are the same as the ones that form the morphodynamic equilibria for $850 \leq B \leq 1800 \mathrm{~m}$. Furthermore, Figure 5.12 reveals that the number of relevant Fourier modes increases for increasing channel width.

\subsection{Discussion}

\subsubsection{Results of LSA of flat bottom as indicators for long- term behavior}

The linear stability analyses (LSA) (Seminara and Tubino, 2001, Schramkowski et al., 2002, Chapters 3 and 4) have provided fundamental knowledge about the mechanisms that explain the initial formation of tidal bars as perturbations of a flat bed. The time evolutions in the previous section allow us to assess the predictive capability of long-term tidal bar patterns by the LSA of the flat bottom. When we compare the pattern that initial forms and the final patterns in the time integration simulations, we observe that in the narrow (1 km wide) channels the wavelength and number of bars in the lateral direction do not change over time. That is, the Fourier mode that initially grows the fastest remains the dominant one in the spectrum in the long-term. So in these cases, the fastest growing pattern from the LSA resembles the final pattern of the time integration reasonably well, although the other Fourier modes are not negligible. However, in the wider channels $(2 \mathrm{~km})$, the dominant tidal bar wavelength in the initial formation (Figure 5.7) is smaller than that in the final 

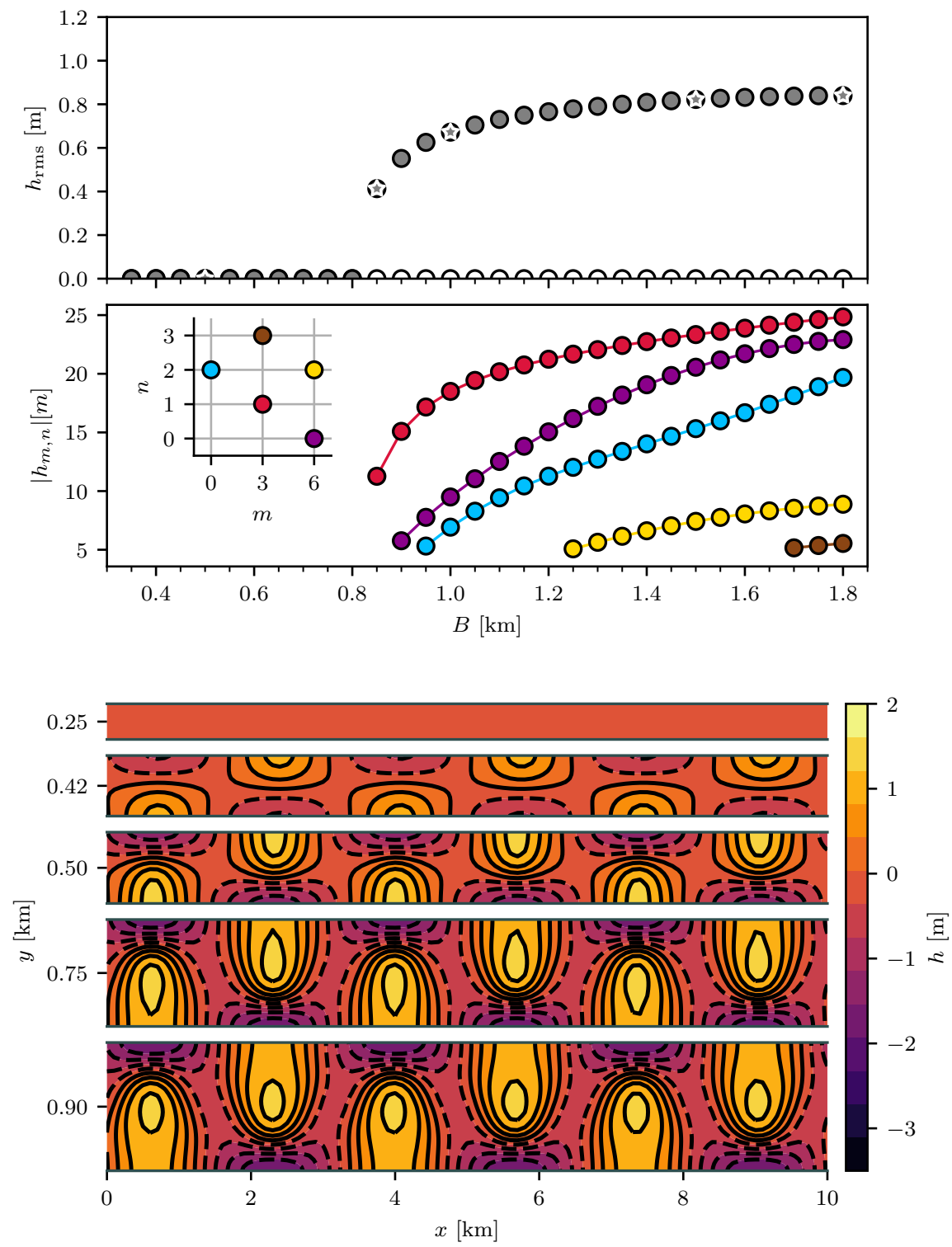

Figure 5.12: Top panel: root-mean square height $h_{\mathrm{rms}}$ of morphodynamic equilibria versus channel width $B$. The gray dots denote stable equilibria, while the white dots represent unstable ones. The patterns corresponding to the dots with a white star are shown in the bottom panels: $B=0.5,0.85,1,1.5,1.8 \mathrm{~km}$. The middle panel shows the amplitudes of the Fourier coefficients of the nontrivial patterns for $B \geq 850 \mathrm{~m}$. The parameter values used are $u_{c}=0, u_{w}=0, \varphi=0^{\circ}, c_{\mathrm{t}}=0$ and the depth $H-h+\zeta$ in $c_{\mathrm{b}}$ is replaced by the undisturbed depth $H$. Other parameter values are as in Table 5.1. 
pattern (Figure 5.10). Furthermore, the bottom has a braided pattern during the initial formation, while in the final pattern bars have merged to form an alternating tidal bar pattern. The difference between the long-term bottom patterns and the fastest growing pattern following from the LSA studies is the largest in the simulation with $B=2 \mathrm{~km}$ and $\varphi=50^{\circ} \mathrm{N}$. Here, the the oblique tidal sand ridges that initially form hardly resemble the final bed forms that show an alternating pattern.

\subsubsection{Comparison with field observations and numerical studies}

Van Veen (1950) described bottom patterns in tidal channels with ebb and flood channels around the bars (see also van den Berg et al., 1996). In the ebb channels the maximum ebb current is stronger than the maximum flood current and vice versa. Such channels were also identified in the numerical results of Hibma et al. (2003). However, the current study seems to lack the mechanism responsible for the formation of such channels. Figure 5.13 shows the locations where the along-channel current is ebb- or flood-dominant at the end of the time integration (after 1000 years). That is, where $\left|\max _{t}(u)\right|-\left|\min _{t}(u)\right|$ is positive (warmer colors) or negative (colder colors). Furthermore, it shows the pattern of the residual currents (arrows). The figure reveals that on the negative $x$-side of the bar the current is flood dominant (red), while at the positive $x$-side it is ebb dominant (blue). So, rather than having ebb or flood dominated channels, the channels are divided in a flood and ebb dominant part. Even though the ebb and flood channels are not present, the structure of the residual current is in agreement with the schematic diagram of estuarine meanders in Ahnert (1960) (Figure 3 in the latter study and Figure 5b of Hibma et al., 2003). In this diagram only at some locations there is a submerged bar between the ebb and flood current trajectories. They attribute the occurrence of the ebb and flood channels to the phase difference between the free surface elevation and the current velocity. In particular, at the locations where the ebb and flood channels occur, there is an overlap in water levels present at high ebb and flood currents (see Figures 6 in both Ahnert (1960) and Hibma et al. (2003)). Due to the local nature (with the rigid-lid assumption) of the current model, this overlap is always there. However, to study the effect of variations in the phase difference between the currents and the free surface elevation, a global model is needed.

The tidal bars simulated in the laboratory by Leuven and Kleinhans (2019) and those observed in the Exe estuary (Figure 1.1), extend over the middle axis of the channel. This corresponds to the results above. Moreover, the laboratory bars in Leuven and Kleinhans (2019) resemble the triangular shape of the bars obtained with our model in $2 \mathrm{~km}$ wide channels (Figure 5.10). However, in the laboratory they do not seem to form by merging two bars on the side and one in the middle of the channel, as observed in the result of the time integration with 


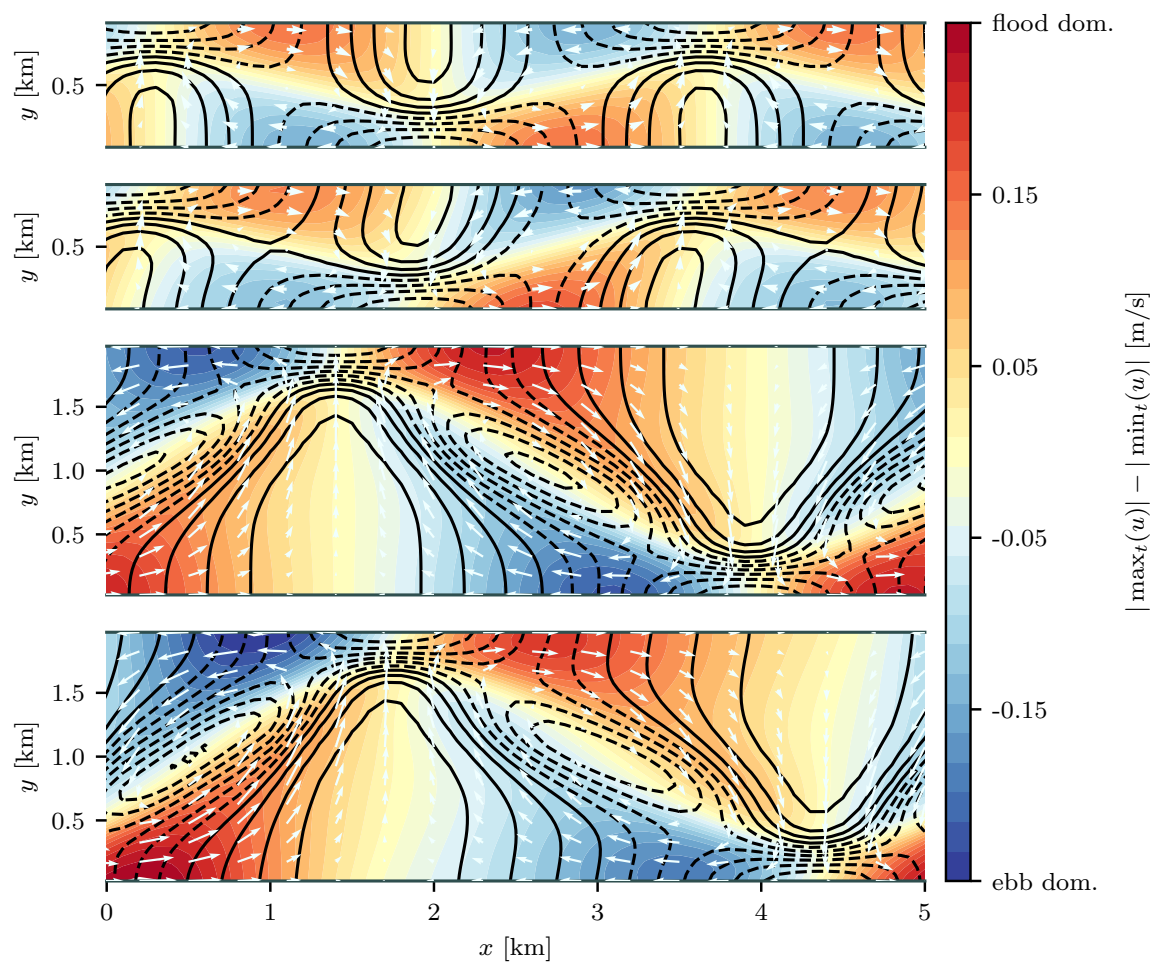

Figure 5.13: Black contour lines denote the bottom height as in Figure 5.9, but for only $0 \leq x \leq 5 \mathrm{~km}$. Dashed lines denote a negative bottom height, while solid lines denote a positive bottom height. The colors denote the (absolute) maximum value of the along-channel current velocity minus the absolute value of its minimum over a tidal cycle. Red colors denote stronger maximum flood currents, while blue colors denote stronger maximum ebb currents. The white arrows depict the spatial distribution of the residual current. 


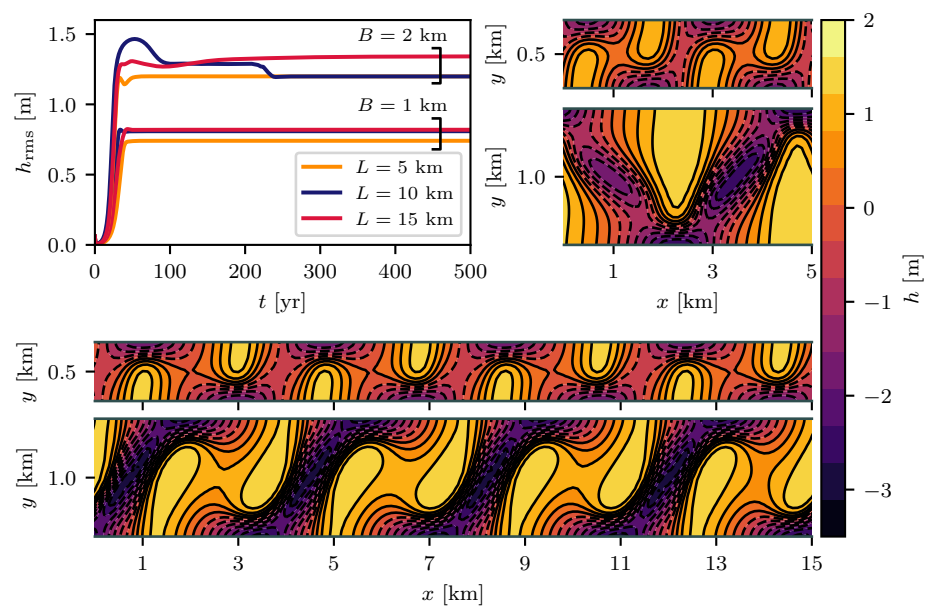

Figure 5.14: Root-mean square height $h_{\mathrm{rms}}$ versus time $t$ for different channel widths $B$ and domain lengths $L$ (top left panel). The dark blue curves $(L=10 \mathrm{~km})$ correspond with those (cyan and red) in Figure 5.6. The top right and bottom panels show the bottom pattern after 500 years for domain lengths of $L=5 \mathrm{~km}$ and $L=15 \mathrm{~km}$, respectively. Parameters are as in Table 5.1.

$B=2 \mathrm{~km}$ and $\varphi=0^{\circ}$. Moreover, the sills connecting the bars, as observed in the laboratory, are not reproduced in the current numerical simulations. Only when the Coriolis effect was taken into account sills formed, but only on one side of the bars.

In the four time integration simulations, the value of the Coriolis parameter seems to be mostly affecting the transient behavior and to a much lesser extent the equilibrium patterns. This is in good correspondence with the fact that the bathymetries both in the numerical studies of, on one hand van der Wegen and Roelvink (2012), who ignore Coriolis, and on the other hand Dam et al. (2016) and Nnafie et al. (2018), who include Coriolis, reproduced the observed bottom topography in the Western Scheldt reasonably well. No clear signature of the Coriolis effect was observed.

\subsubsection{Model limitations}

The first important limitation of the model in this study is that it does not allow for intertidal areas, while natural tidal bars often are only submerge during a part of the tidal cycle. The second limitation is that the contribution of the free surface elevation due to the external pressure gradient on the water depth is in many systems significant, whereas in this model only the free surface elevation as a result of the bottom height is considered. These two choices are 
numerically rather than physically motivated and therefore deserve additional attention. A third limitation is the dependency of the model results on the choice of the domain length $L$. When $L$ is too long, the assumption of alongchannel uniformity is violated. However, when the domain length $L$ is too short, the number of along-channel Fourier modes is strongly restricted thereby limiting the dynamics. Figure 5.14 shows the root-mean square height and the bottom pattern after 500 years for a channel domain of $L=5$ and $L=15 \mathrm{~km}$ ( $\pm 50 \%$ of the default channel length). The channels are 1 and $2 \mathrm{~km}$ wide and the latitude $\varphi=50^{\circ} \mathrm{N}$. The comparison of the result in Figure 5.10 with those in Figure 5.14 reveals that the results in a 5, 10 or $15 \mathrm{~km}$ long channel agree quantitatively with those obtained in the $1 \mathrm{~km}$ wide channel. Quantitatively, the root-mean square height and the along-channel wavelength are slightly different. In the $2 \mathrm{~km}$ wide channel, the results show a stronger sensitivity to the choice of channel length. When the channel length is halved $(L=5 \mathrm{~km})$, the time interval in which the root-mean square height temporary stopped changing, as in the simulation with $L=10 \mathrm{~km}$ (between 100 and 200 years), is not present anymore. When the channel length is increased by $50 \%(L=15 \mathrm{~km})$, the rapid adjustment, as observed around 250 years in the simulation of $L=10 \mathrm{~km}$, is not found in the first 500 years of simulation. In fact, the pattern after 500 years in the long channel $(15 \mathrm{~km})$ resembles that of the original simulation $(L=10 \mathrm{~km})$ after 150 years (bottom panel Figure 5.9. It is unclear whether this pattern will eventually also change to a pattern similar
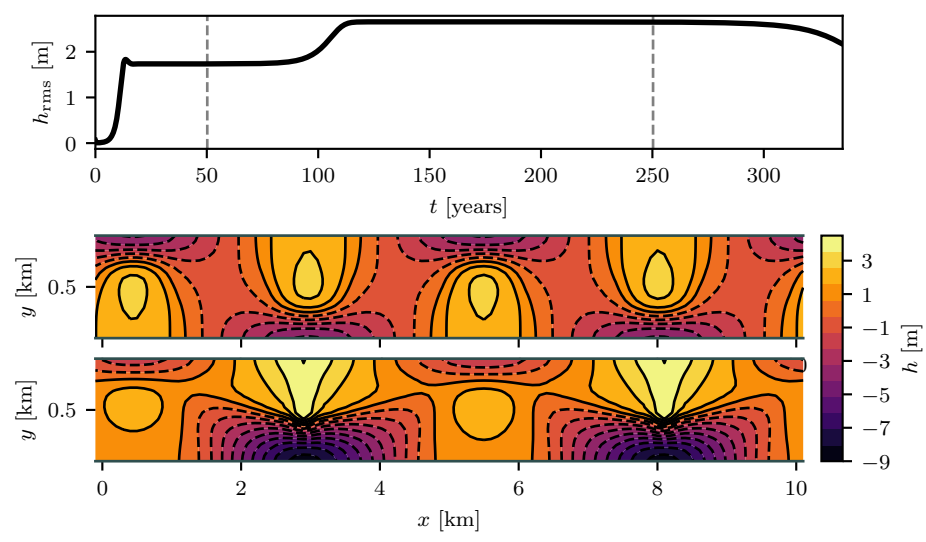

Figure 5.15: Top panel shows the root-mean square height $h_{\mathrm{rms}}$ versus time $t$. The bottom panels show the bottom height versus space after 50 and 250 years, corresponding to the vertical gray lines in the top panel. The solid black line in Figure 5.15 corresponds with the black line in the top panel. The simplified model, as described in the validation section of 5.2.6, is used with parameters values as in Table 5.1 with $B=1000 \mathrm{~m}, U=1 \mathrm{~m} / \mathrm{s}, H=5 \mathrm{~m}, u_{c}=0, u_{w}=0, \mu=1 \mathrm{~m}^{2} \mathrm{~s}^{-1}$ and $\varphi=0^{\circ}$. 
to the one in the $L=10 \mathrm{~km}$ simulation (bottom panel in Figure 5.10) if the simulation time would be increased.

A further systematic sensitivity analysis to model parameters is needed before robust conclusions can be made about timescales at which patterns settle in a morphodynamical equilibrium and their associated final heights. Figure 5.15 reveals that in different regions of the parameter space, the dynamics can be vastly different. The top panel shows the time evolution of the root-mean square height of the bottom for the simulation used in Figure 5.5 (i.e., the simplified model as discribed in the verification section in 5.2.6). The bottom panels show the patterns after 50 and 250 years. As in the simulation with $B=2 \mathrm{~km}$ and $\varphi=50^{\circ}$, the bottom height is almost constant after an initial adjustment (in this case after a few decades), but then (after a few centuries) changes drastically again. After 350 years the model crashed (likely due to a numerical instability). The pattern after 250 years is characterized by an asymmetric distribution of sediments with respect to the channel axis; most sediments are deposited on left side of the channel (viewed in the positive $x$ direction). This is another example where the system seems to have found an equilibrium (twice), but then quickly evolves after some time. Other possible extensions of the model are to consider multiple tidal constituents in the tidal forcing and to include the role of river runoff, wind waves, vegetation, sand-mud mixtures and stratification on the hydro- and morphodynamics.

\subsection{Conclusions}

The long-term dynamics of tidal bars in a confined tidal channel is studied with a numerical model of intermediate complexity. Its formulation allows a comparison of the bottom evolution with that predicted by linear stability analyses of a flat bottom. The evolution of the bathymetry starting from a randomly perturbed flat bottom is simulated over a few centuries for tidal channels of different widths and at different latitudes. Eventually, in all simulations the tidal bars stopped evolving after a certain amount of time. Also, in all simulations the tidal bars extended over the middle axis of the channel; a characteristic also observed in nature and in the laboratory. The differences between the simulations yields a first insight in the sensitivity of the tidal bar dynamics to channel width and the Coriolis effect. In the $1 \mathrm{~km}$ wide channels, the initially forming patterns are only slightly adjusted over time (by extending over the middle axis). The Coriolis effect only slightly skews this pattern, thereby forming shallow sills between the bars. In the $2 \mathrm{~km}$ wide channels the dynamics is richer. The initially braiding pattern evolves in an alternating bar pattern by merging tidal bars in the middle of the channel with those on the side, thereby also increasing the tidal bar wavelength. Moreover, in the $2 \mathrm{~km}$ wide channel located at a latitude of $50^{\circ} \mathrm{N}$, the bars seem to reach a morphodynamic equilibrium after a century, but rapidly change, after approximately 2.5 centuries, 
into a drastically different pattern. The pattern observed after a century is very different from the pattern in the $2 \mathrm{~km}$ wide channel when the Coriolis effect was neglected. However, the pattern after 2.5 centuries is, like the one observed in the $1 \mathrm{~km}$ wide channel merely a skewed version of the case with the channel located at latitude $0^{\circ}$. This highlights one of the the difficulties of studying equilibrium tidal bar patterns by means of a time integration; it is unclear when and if a pattern is in morphodynamic equilibrium. A numerical continuation shows the morphodynamic equilibria for different channel widths. For increasing widths, the bars extend further over the channel axes and the maximum bottom height of the patterns moves away from the channel boundary. 


\section{Summary and outlook}

The overarching goal of the thesis is to contribute to the understanding of the dynamics of tides and tidal bars in tidal channels. Below, a summary is presented in which the main findings of the preceding chapters and suggestions for future research are given.

\subsection{Summary}

\subsubsection{Objective 1}

The aim of Chapter 2 was to understand the sensitivity of tidal characteristics (such as times of high and low water, tidal range, ratio between maximum flood and ebb currents, etc.) to momentum dissipation on tidal flats. When the tide rises, water flows from the channel onto the adjacent tidal flats, carrying with it longitudinal momentum. Some hours later, when the tides falls, water flows back into the channel. However, its longitudinal momentum is by then largely dissipated due to high friction on the tidal flats. A model was developed and analysed that describes tides in an open channel with tidal flats along its sides. The water motion is forced by $\mathrm{M}_{2}$ tidal elevations at both open ends. By assuming these elevations to be small compared to the water depth and the width of flats of the same order as the width of the main channel, approximate solutions of the model could be constructed by means of a perturbation analysis. It was found that this so-called momentum sink decreases the $\mathrm{M}_{2}$ amplitude of both the current velocity and the sea surface elevation and favours flood dominant tides. The decrease of the $\mathrm{M}_{2}$ amplitudes is caused by the fact that, during the fall of the tide, still water enters the channel and slows down the 
current. The finding that the momentum sink favors flood-dominant tides is explained by noting that the momentum sink acts as an advective term during the fall of the tide, which is known to favour flood currents. As a result, the momentum sink increases the $\mathrm{M}_{4}$ amplitudes in a flood-dominant system. The impact of the momentum sink on tidal characteristics becomes larger with increasing ratio of intertidal area and channel area. Moreover it is, to a much lesser extent, sensitive to the slope of the flats, drag coefficient, embayment length and the phase difference between incoming tidal waves at the boundary of the embayment.

\subsubsection{Objective 2}

After the discussion about the dissipation of momentum on tidal flats, the attention turned to the initial formation of tidal bars. In literature, there was an inconsistency about the possible dependence of the tidal bar wavelength on channel width. Observations and numerical models showed this dependency. However, Leuven et al. (2016) showed that the semi-analytical models of Schramkowski et al. (2002) and Seminara and Tubino (2001), which were intended to explain the initial formation of tidal bars, could not reproduce this dependency. The aim of Chapter 3 was therefore to extend the model of Schramkowski et al. (2002) such that the tidal bar wavelength of the fastest growing pattern (the preferred wavelength) does depend on channel width, while still roughly reproducing natural (observed) tidal bar patterns.

The key extension was to include the effects of horizontal eddy diffusivity and in particular the resulting bed slope effect. The reason the extended model shows a clear dependence of tidal bar wavelength on channel width, whilst in the original model of Schramkowski et al. (2002) this dependence was weak, is the following. First of all, in wider channels, the pattern with the largest growth rate (the preferred tidal bar pattern) has more bars in the lateral direction. That is, it has a higher preferred mode number. Second, in the range of channel widths with constant preferred mode number, the preferred wavelength increases with increasing channel width. However, at the width where the preferred mode number changes (it increases by one), the preferred wavelength decreases. In the model of Schramkowski et al. (2002), the range of channel widths with constant preferred mode number is relatively short. This means that, with increasing channel width, before the preferred wavelength can increase significantly, the preferred mode number increases and with that, the wavelength decreases again. As a result, the tidal bar wavelength remains of the same order of magnitude for different values of channel width. In the present study, the effects of horizontal eddy diffusivity are added to the model. As a result, the effective bed slope effect is stronger. Since bed slope effects decrease the growth rate of bottom patterns with large gradients, the bottom pattern with the largest growth rate has a smaller mode number. This implies that the preferred mode number remains the same for a wider range of channel 
widths than in the model of Schramkowski et al. (2002). The width dependence now follows from the fact that, in this range, the tidal bar wavelength increases with increasing channel width and that this increase is stronger for smaller mode numbers.

A sensitivity analysis confirmed the finding of earlier studies that the tidal bar wavelength depends on the current velocity, the channel depth and the channel width. Moreover, an approximate expression of the tidal bar wavelength, in terms of three internal length scales (channel width, tidal excursion length, friction length scale), revealed that when one of the length scales is very small (large), the sensitivity of the tidal bar wavelength to that length scale is large (small) compared to the others.

\subsubsection{Objective 3}

Besides the effects of horizontal turbulent exchange of sediments, also the role of the Coriolis effect on the initial formation of tidal bars was not studied previously. However, tidal bars are present in systems for which the Rossby number is close to one, implying that the Coriolis effect should be taken into account. Moreover, results of a numerical model showed that the initial formation of tidal bars is sensitive to the value of the Coriolis parameter. The aim of Chapter 4 was to study the role of the Coriolis effect on the initial formation of bottom patterns in a tidal channel. Using the insights gained in the study to the channel width dependence, a new model was formulated that accounts for the Coriolis effect on the hydrodynamics. The key finding is that the mechanism generating oblique tidal sand ridges on the continental shelf is also present in confined tidal channels. When the inverse Rossby number exceeds values of approximately 0.5 , this causes the fastest growing pattern to be a combination of tidal bars and oblique tidal sand ridges. Moreover, it causes the pattern to grow faster, without significantly altering the preferred wavelength.

\subsubsection{Objective 4}

After the two studies of the initial formation of tidal bars, the aim of Chapter 5 concerned the long-term evolution of tidal bars. This long-term evolution was previously studied in laboratory settings and in numerical models. Most of the numerical models are so-called global models; they simulate an entire channel from sea to the landward (or river) boundary. In contrast to global models, simulate local models only a short section of a long channel, which makes them easier to analyse. Only one of the available numerical models was a local model. However, this local model was restricted to small friction parameters. This motivated the fourth objective, which was to investigate the long-term dynamics of tidal bars in realistic parameter regimes with a local model. In particular, the presence of nontrivial morphodynamic equilibria was studied, the long-term patterns compared with those that initially form and 
the sensitivity of the results to the Coriolis parameter and channel width was assessed. To this end, a new numerical model of intermediate complexity was developed. This model aimed to bridge the gap between the local models that study the initial formation, the local model that was restricted to weak friction and the global numerical models that study the long-term dynamics. The model domain is periodic in the along-channel direction and represents a section of a tidal channel of approximately $3 \mathrm{~m}$ deep, $1-2 \mathrm{~km}$ wide and with currents of approximately $0.5 \mathrm{~m} \mathrm{~s}^{-1}$ (roughly representing the Exe estuary).

Four time integration runs showed that, for the parameter values considered (including realistic values of the friction parameter), the bottom height seems to stop evolving after a certain time. These final patterns showed similarities with the results of global numerical studies, observations in nature and bars simulated in a laboratory. In particular, the matured bars exceed the middle axis of the channel. The Coriolis effect only moderately affects these longterm patterns. However, the transient dynamics is sometimes vastly altered by the Coriolis effect. Moreover, the results reveal that for some parameter settings (in particular, a channel width of $1 \mathrm{~km}$ ), the initial formation, as predicted by the linear stability analyses in the previous chapters, resembles the long-term patterns reasonably well, while in other cases the long-term pattern differs strongly from the one that forms initially (in the $2 \mathrm{~km}$ wide channels). Furthermore, the results highlight difficulties with studying morphodynamic equilibria by means of a time integration methodology: it is unclear when and if a pattern is converged to a morphodynamic equilibrium. As an alternative, a root-finding algorithm was used to find morphodynamic equilibria for different channel widths. For increasing channel widths, the bars extend further over the channel axes and the maximum bottom height of the patterns moves away from the channel boundary. The results are preliminary, but serve as a proof of concept to further systematically investigate the tidal bar dynamics in different parameter regimes.

\subsection{Outlook}

Below, a few options for further research are suggested.

In Chapter 2, the model domain was chosen such that the depth and width of the main channel were constant. There are many natural channels where this is not a realistic assumption. An extension could therefore be to consider a channel geometry that includes depth and width variations in the along-channel direction. Moreover, the effect of different tidal constituents at the boundary could be included and different scaling choices could be explored. The latter could be done, for example, by making different choices about the size and shape of the tidal flats. Furthermore, it would be interesting to compare the results with those of the $2 \mathrm{D}$ model by Boelens et al. (2018).

In Chapter 3-5, tidal channels are considered in which stratification due 
to vertical differences in water density is negligible. Olabarrieta et al. (2018) showed that in stratified channels, the bottom patterns are strongly affected by the density-driven dynamics. It would be interesting to see if this could be further investigated in a mathematically tractable model. For this a 3D model is needed, because the density-driven dynamics requires a vertical dimension and the bottom patterns discussed in this thesis require two horizontal dimensions. Moreover, in the models presented in Chapter 3-5, the sides of the channel are not erodible. However, many natural channels have sides that are erodible and it is known that the evolution of bottom patterns and of erodible sides (meandering) becomes coupled (Solari et al., 2002). After Chapter 4, it would be interesting to study the role of the Coriolis force in such a system. To account for transverse circulations induced by channel curvature and the Coriolis effect, also in this case, a 3D model is needed. In addition to modelling studies, laboratory experiments, such as those performed by Leuven and Kleinhans (2019), in a meandering channel could also be insightful.

The results in Chapter 5 were preliminary and suggest multiple options to further research. First of all, the model settings in the time integration runs and the continuation runs should be made consistent. A second option is to perform more numerical continuation experiments of morphodynamic equilibria for different bifurcation parameters. Here, only the channel width was varied and only up to $1.8 \mathrm{~km}$. However, the results of the time integration runs of a channel width of $2 \mathrm{~km}$ (Figure 5.10) suggest that the pattern as in the bottom panel of Figure 5.12 becomes unstable when the channel width exceeds a certain value. The methodology described in Chapter 5 allows one to perform a linear stability analysis of the unstable equilibrium. This could explain why the finger shaped tidal bar patterns as shown in Figure 5.12 would change to the more triangular shaped patterns in Figure 5.10.

Moreover, in the discussion of Chapter 5 (Section 5.4) the effect of the domain length $L$ on the results is briefly discussed. Since, the choice of the domain length is rather arbitrary, this deserves more attention. This could also be done by means of a continuation. Furthermore, the effect of other model parameters (that do not change the numerical grid) on the spatial patterns of morphodynamic equilibria could be investigated by means of a pseudo-arc length continuation (e.g, using BifurcationKit.jl). Examples of possibly interesting parameters to vary are the latitude $\varphi$, the drag coefficient $c_{d}$ and the critical velocity for erosion $u_{c}$. Concerning the latitude $\varphi$, one could aim to answer the question why the signature of the Coriolis effect present in the initial formation, disappears after a while. Is there another mechanism that overtakes the one responsible for the ridge like structure?

Numerically there is also possibly room for improvement. The current code integrates the hydrodynamics in time by means of a Runge-Kutta 4 scheme with fixed time steps of 5 seconds. This is computationally the most expensive part of the code. Perhaps, other time integration methods can speed up these calculations. To experiment with implicit time integrators (implemented in 
DifferentialEquations.jl), the automatic differentiation package ForwardDiff.jl could also be used here. Another option would be to solve the (spatially discretized) continuity, momentum and concentration equation(s) by means of spectral methods (here ApproxFun.jl could potentially be useful). Lastly, the Julia language makes the investment to adapt the model to run on GPUs or in parallel relatively cheap, so perhaps this could also be a fruitful thing to try. Once such a model is fast enough, it would be interesting to extent it to a global (rather than local) model which is still capable of performing a numerical bifurcation analysis. 


\section{Bibliography}

Ahnert, F. (1960). Estuarine meanders in the Chesapeake Bay area. Geographical Review, 50(3):390-401.

Alebregtse, N. C. (2015). Modeling the hydrodynamics in tidal networks. PhD thesis, Utrecht University.

Aubrey, D. G. (1986). Hydrodynamic controls on sediment transport in wellmixed bays and estuaries. In van de Kreeke, J., editor, Physics of Shallow Estuaries and Bays, volume 16 of Lecture Notes on Coastal and Estuarine Studies, pages 245-258. American Geophycisal Union.

Baar, A. W., de Smit, J., Uijttewaal, W. S. J., and Kleinhans, M. G. (2018). Sediment transport of fine sand to fine gravel on transverse bed slopes in rotating annular flume experiments. Water Resources Research, 54(1):19-45.

Bagnold, R. A. (1963). Mechanics of marine sedimentation. In Hill, M. N., editor, The Sea: Ideas and Observations, volume 3. Wiley-Ineterscience, New York.

Bailard, J. A. (1981). An energetics total load sediment transport model for a plane sloping beach. Journal of Geophysical Research: Oceans, 86(C11):10938-10954.

van den Berg, J. H., Jeuken, C. J. L., and van der Spek, A. J. F. (1996). Hydraulic processes affecting the morphology and evolution of the Westerschelde estuary, chapter 7. Esutarine Shores: Evolution, Enviornments and Human Alterations. John Wiley \& Sons.

Bezanson, J., Edelman, A., Karpinski, S., and Shah, V. B. (2017). Julia: A fresh approach to numerical computing. SIAM Review, 59(1):65-98. 
Boelens, T., Schuttelaars, H., Schramkowski, G., and De Mulder, T. (2018). The effect of geometry and tidal forcing on hydrodynamics and net sediment transport in semi-enclosed tidal basins. Ocean Dynamics, 68(10):1285-1309.

Boon, J. D. and Byrne, R. J. (1981). On basin hypsometry and the morphodynamic response of coastal inlet systems. Marine Geology, 40:27-48.

Bouma, H., de Jong, D. J., Twisk, F., and Wolfstein, K. (2005). A Dutch ecotope system for coastal waters. Technical Report 2005.024, Rijksinstituut voor Kust en Zee.

ter Brake, M. C. and Schuttelaars, H. M. (2010). Modeling equilibrium bed profiles of short tidal embayments. Ocean Dynamics, 60(2):183-204.

ter Brake, M. C. and Schuttelaars, H. M. (2011). Channel and shoal development in a short tidal embayment: an idealized model study. Journal of Fluid Mechanics, 667:503-529.

Brown, J. M. and Davies, A. G. (2010). Flood/ebb tidal asymmetry in a shallow sandy estuary and the impact on net sand transport. Geomorphogly, 114:431-439.

Buijsman, M. C. and Ridderinkhof, H. (2007). Long-term ferry-adcp observations of tidal currents in the Marsdiep inlet. Journal of Sea Research, $57: 237-256$.

Caballeria, M., Coco, G., Falqués, A., and Huntley, D. A. (2002). Selforganization mechanisms for the formation of nearshore crescentic and transverse sand bars. Journal of Fluid Mechanics, 465:379-410.

Cancino, L. and Neves, R. (1999). Hydrodynamic and sediment suspension modelling in estuarine systems: Part II: Application to the Western Scheldt and Gironde estuaries. Journal of Marine Systems, 22(2):117-131.

Crosato, A. and Mosselman, E. (2009). Simple physics-based predictor for the number of river bars and the transition between meandering and braiding. Water Resources Research, 45(3).

Dalrymple, R. W. and Choi, K. (2007). Morphologic and facies trends through the fluvial-marine transition in tide-dominated depositional systems: A schematic framework for environmental and sequence-stratigraphic interpretation. Earth-Science Reviews, 81(3):135 - 174.

Dalrymple, R. W. and Rhodes, R. M. (1995). Estuarine dunes and bars. In Perillo, G., editor, Geomorphology and Sedimentology of Estuaries. Developments in Sedimentology, pages 359-422. Elsevier Science B.V. 
Dam, G., van der Wegen, M., Labeur, R. J., and Roelvink, D. (2016). Modeling centuries of estuarine morphodynamics in the Western Scheldt estuary. Geophysical Research Letters, 43(8):3839-3847.

David, L. T. and Kjerfve, B. (1998). Tides and currents in a two-inlet coastal lagoon: Laguna de Términos, México. Continental Shelf Research, 18:10571079 .

Deltares (2019). User manual Delft3d-Flow: Simulation of multi-dimensional hydrodynamic flows and transport phenomena, including sediments (version 3.15). Delft, The Netherlands.

Dijkstra, H. A., Wubs, F. W., Cliffe, A. K., Doedel, E., Dragomirescu, I. F., Eckhardt, B., Gelfgat, A. Y., Hazel, A. L., Lucarini, V., Salinger, A. G., and et al. (2014). Numerical bifurcation methods and their application to fluid dynamics: Analysis beyond simulation. Communications in Computational Physics, 15(1):1-45.

Dodd, N., Blondeaux, P., Calvete, D., de Swart, H. E., Falqués, A., Hulscher, S. J. M. H., Różyński, G., and Vittori, G. (2003). Understanding coastal morphodynamics using stability methods. Journal of Coastal Research, 19(4):849-865.

Dronkers, J. (2005). Dynamics of coastal systems. World Scientific Publishing Co. Pte. Ltd.

Dronkers, J. J. (1964). Tidal computations in rivers and coastal waters. Amsterdam: North-Holland Publishing Co.

Dronkers, J. J. and Schönfeld, J. C. (1959). Tidal computations in rivers and coastal waters. In Waalewijn, A., editor, Report on hydrostatic levelling across the Westerschelde, number 1 . Rijkswaterstaat.

Duran-Matute, M., Gerkema, T., de Boer, G. J., Nauw, J. J., and Gräwe, U. (2014). Residual circulation and freshwater transport in the Dutch Wadden Sea: a numerical modelling study. Ocean Science, 10:611-632.

Dyer, K. R. (1986). Coastal and Estuarine Sediment Dynamics. John Wiley \& Sons.

Engelund, F. and Hansen, E. (1967). A monograph on sediment transport in alluvial streams. Technical report, Teknisk Forlag.

Friedrichs, C. T. (2011). Tidal flat morphodynamics: a synthesis. In Hansom, J. D. and Fleming, B. W., editors, Treatise on Estuarine and Coastal Science, volume 3: Estuarine and Coastal Geology and Geomorphology, pages 137170. Elsevier. 
Friedrichs, C. T. and Aubrey, D. G. (1988). Non-linear tidal distortion in shallow well-mixed estuaries, a synthesis. Estuarine, Coastal and Shelf Science, $27: 521-545$.

Friedrichs, C. T. and Aubrey, D. G. (1994). Tidal propagation in strongly convergent channels. Journal of Geophysical Research., 99:3321-3336.

Garnier, R., Calvete, D., Falques, A., and Caballeria, M. (2006). Generation and nonlinear evolution of shore-oblique/transverse sand bars. Journal of Fluid Mechanics, 567:327-360.

Garotta, V., Bolla Pittaluga, M., and Seminara, G. (2006). On the migration of tidal free bars. Physics of Fluids, 18(9):096601.

Gerkema, T. (2019). An Introduction to Tides. Cambridge University Press.

Glanzmann, C. F., Blenne, B., and Burgess, F. J. (1971). Tidal hydraulics, flushing characteristics and water quality of Netarts bay. Technical report, Engineering Experiment Station, Oregon State University, Corvallis, Oregon.

Griewank, A. and Walther, A. (2008). Evaluating derivatives. Principles and techniques of Algorithmic Differentiation. Society for Industiral and Applied Mathematics (SIAM), 2nd edition.

Groen, P. (1967). On the residual transport of suspended matter by an alternating tidal current. Netherlands Journal of Sea Research, 3:564-574.

Hepkema, T. M., de Swart, H. E., Zagaris, A., and Duran-Matute, M. (2018). Sensitivity of tidal characteristics in double inlet systems to momentum dissipation on tidal flats: a perturbation analysis. Ocean Dynamics, 68(4):439 455.

Herrmann, R. and Hübner, D. (1982). Behaviour of polycyclic aromatic hydrocarbons in the Exe estuary, Devon. Netherlands Journal of Sea Research, $15(3): 362-390$.

Hibma, A., de Vriend, H., and Stive, M. (2003). Numerical modelling of shoal pattern formation in well-mixed elongated estuaries. Estuarine, Coastal and Shelf Science, 57(5):981 - 991.

Hibma, A., Schuttelaars, H. M., and de Vriend, H. J. (2004). Initial formation and long-term evolution of channel-shoal patterns. Continental Shelf Research, 24:1637-1650.

Huthnance, J. M. (1982). On one mechanism forming linear sand banks. Estuarine, Coastal and Shelft Science, 14:79-99.

IPCC (2019). Special report on the ocean and cryoshpere in a changing climate. https://www.ipcc.ch/srocc/. 
van de Kreeke, J. and Robaczewska, K. (1993). Tide-induced residual transport of coarse sediment; application to the Ems estuary. Netherlands Journal of Sea Research, 31:209-220.

Krol, M. (1991). On the averaging method in nearly time-periodic advectiondiffusion problems. SIAM Journal on Applied Mathematics, 51(6):1622-1637.

Kuznetsov, Y. (2004). Elements of Applied Bifurcation Theory, volume 112. Springer-Verlag New York.

van de Lageweg, W. and Feldman, H. (2018). Process-based modelling of morphodynamics and bar architecture in confined basins with fluvial and tidal currents. Marine Geology, 398:35-47.

Leuven, J. R. F. W. and Kleinhans, M. G. (2019). Incipient tidal bar and sill formation. Journal of Geophysical Research: Earth Surface, 124(7):17621781.

Leuven, J. R. F. W., Kleinhans, M. G., Weisscher, S. A. H., and van der Vegt, M. (2016). Tidal sand bar dimensions and shapes in estuaries. Earth-Science Reviews, 161:204-223.

Lorentz, H. A. (1922). Het in rekening brengen van den weerstand bij schommelende vloeistofbewegningen. De Ingenieur, 37:695-696.

Nnafie, A., Van Oyen, T., De Maerschalck, B., van der Vegt, M., and van der Wegen, M. (2018). Estuarine channel evolution in response to closure of secondary basins: An observational and morphodynamic modeling study of the Western Scheldt estuary. Journal of Geophysical Research: Earth Surface, 123(1):167-186.

Olabarrieta, M., Geyer, W. R., Coco, G., Friedrichs, C. T., and Cao, Z. (2018). Effects of density-driven flows on the long-term morphodynamic evolution of funnel-shaped estuaries. Journal of Geophysical Research: Earth Surface, 123(11).

Parker, B. B. (1984). Frictional effects on the tidal dynamics of a shallow estuary. $\mathrm{PhD}$ thesis, University Baltimore.

Parker, B. B., editor (1991). Tidal hydrodynamics. Wiley, New York.

Press, W. H., Teukolsky, S. A., Vetterling, W. T., and Flannery, B. P. (2007). Numerical Recipes: The Art of Scientific Computing. Cambridge University Press, 3rd edition.

Rackauckas, C. and Nie, Q. (2017). Differentialequations.jl-a performant and feature-rich ecosystem for solving differential equations in julia. Journal of Open Research Software, 5(1). 
Revels, J., Lubin, M., and Papamarkou, T. (2016). Forward-mode automatic differentiation in julia. CoRR.

Ridderinkhof, H. (1988). Tidal and residual flows in the Western Dutch Wadden Sea I: Numerical model results. Netherlands Journal of Sea Research, 22:121.

Ridderinkhof, W., de Swart, H. E., van der Vegt, M., Alebregtse, N. C., and Hoekstra, P. (2014). Geometry of tidal basin systems: A key factor for the net sediment transport in tidal inlets. Journal of Geophysical Research: Oceans, 119:6988-7006.

Roos, P. C., Hulscher, S. J. M. H., Knaapen, M. A. F., and Van Damme, R. M. J. (2004). The cross-sectional shape of tidal sandbanks: Modeling and observations. Journal of Geophysical Research: Earth Surface, 109(F2).

Roos, P. C. and Schuttelaars, H. M. (2015). Resonance properties of tidal channels with multiple retention basins: role of adjacent sea. Ocean Dynamics, $65: 311-324$.

Royal Netherlands Navy, Hydrographic Service (2016). HP33 Tidal heights and streams along the Netherlands coast and adjacent areas. .

Schramkowski, G. P., Schuttelaars, H. M., and de Swart, H. E. (2002). The effect of geometry and bottom friction on local bed forms in a tidal embayment. Continental Shelf Research, 22(11):1821 -1833. Proceedings from the Tenth Biennial Conference on the Physics of Estuaries and Coastal Seas.

Schramkowski, G. P., Schuttelaars, H. M., and de Swart, H. E. (2004). Nonlinear channel-shoal dynamics in long tidal embayments. Ocean Dynamics, 54(3):399-407.

Schuttelaars, H. M. and de Swart, H. E. (1999). Initial formation of channels and shoals in a short tidal embayment. Journal of Fluid Mechanics, 386:1542 .

Seminara, G. and Tubino, M. (2001). Sand bars in tidal channels. Part 1. Free bars. Journal of Fluid Mechanics, 440:49-74.

Serrano, D., Ramírez-Félix, E., and Valle-Levinson, A. (2013). Tidal hydrodynamics in a two-inlet coastal lagoon in the Gulf of California. Continental Shelf Research, 63:1-12.

Shampine, L. F. (2005). Solving odes and ddes with residual control. Applied Numerical Mathematics, 52(1):113-127.

Smith, J. D. and McLean, S. R. (1977). Spatially averaged flow over a wavy surface. Journal of Geophysical Research, 82(12):1735-1746. 
Solari, L., Seminara, G., Lanzoni, S., Marani, M., and Rinaldo, A. (2002). Sand bars in tidal channels part 2. tidal meanders. Journal of Fluid Mechanics, 451:203-238.

Soulsby, R. (1997). Dynamics of marine sands. Thomas Telford.

Speer, P. E. (1984). Tidal Distortion in Shallow Estuaries. PhD thesis, Woods Hole Oceanographic Institution Massachusetts Institute of Technology.

Speer, P. E. and Aubrey, D. G. (1985). A study of non-linear tidal propagation in shallow inlet/estuarine systems Part II: Theory. Estuarine, Coastal and Shelf Science, 21:207-224.

Struiksma, N., Olesen, K. W., Flokstra, C., and de Vriend, H. J. (1985). Bed deformation in curved alluvial channels. Journal of Hydraulic Research, 23(1):57-79.

de Swart, H. E., Alebregtse, N. C., and Zimmerman, J. T. F. (2011). Tidal asymmetry in basins due to channel-flat interactions, simple model. In Shao, X., Wang, Z., and Wang, G., editors, Proceedings of the 7th IAHR Symposium on River, Coastal and Estuarine Morphodynamics, pages 523-531. Tsinghua University Press.

de Swart, H. E. and Zimmerman, J. T. F. (2009). Morphodynamics of tidal inlet systems. Annual Review of Fluid Mechanics, 41:203-29.

Talke, S. A. and Jay, D. A. (2020). Changing tides: The role of natural and anthropogenic factors. Annual Review of Marine Science, 12(1):121-151.

Tambroni, N., Ferrarin, C., and Canestrelli, A. (2010). Benchmark on the numerical simulations of the hydrodynamic and morphodynamic evolution of tidal channels and tidal inlets. Continental Shelf Research, 30(8):963983.

Tambroni, N., Pittaluga, M. B., and Seminara, G. (2005). Laboratory observations of the morphodynamic evolution of tidal channels and tidal inlets. Journal of Geophysical Research: Earth Surface, 110(F04009).

Terra, G. M., van de Berg, W. J., and Maas, L. R. M. (2005). Experimental verification of Lorentz' linearization procedure for quadratic friction. Fluid Dynamics Research, 36:175-188.

Valle-Levinson, A. (2008). Density-driven exchange flow in terms of the Kelvin and Ekman numbers. Journal of Geophysical Research: Oceans, 113(C4).

van Veen, J. (1950). Eb- en vloedschaarsystemen in de nederlandse getijwateren. Tijdschrift Koninklijk Nederlands Aardrijkskundig Genootschap, 67:303-325. 
Veltz, R. (2020). BifurcationKit.jl. https://hal.archives-ouvertes.fr/ hal- 02902346.

van der Wegen, M. (2013). Numerical modeling of the impact of sea level rise on tidal basin morphodynamics. Journal of Geophysical Research: Earth Surface, 118(2):447-460.

van der Wegen, M. and Roelvink, J. A. (2008). Long-term morphodynamic evolution of a tidal embayment using a two-dimensional, process-based model. Journal of Geophysical Research: Oceans, 113(C03016).

van der Wegen, M. and Roelvink, J. A. (2012). Reproduction of estuarine bathymetry by means of a process-based model: Western Scheldt case study, the Netherlands. Geomorphology, 179:152 - 167.

Winant, C. D. (2008). Three-dimensional residual tidal circulation in an elongated, rotating basin. Journal of Physical Oceanography, 38(6):1278-1295.

Wolanski, E., Moore, K., Spagnol, S., D’Adamo, N., and Pattiaratchi, C. (2001). Rapid, human-induced siltation of the macro-tidal Ord River estuary, Western Australia. Estuarine, Coastal and Shelf Science, 53(5):717-732.

Xie, D., Gao, S., Wang, Z. B., Pan, C., Wu, X., and Wang, Q. (2017). Morphodynamic modeling of a large inside sandbar and its dextral morphology in a convergent estuary: Qiantang estuary, china. Journal of Geophysical Research: Earth Surface, 122(8):1553-1572.

Yuan, B., de Swart, H. E., and Panadès, C. (2016). Sensitivity of growth characteristics of tidal sand ridges and long bed waves to formulations of bed shear stress, sand transport and tidal forcing: A numerical model study. Continental Shelf Research, 127:28 - 42.

Zimmerman, J. T. F. (1981). Dynamics, diffusion and geomorphological significance of tidal residual eddies. Nature, 290:549-555.

Zimmerman, J. T. F. (1982). On the lorentz linearization of a quadratically damped forced oscillator. Physics letters, 89A(3):123-124. 


\section{Nederlandse samenvatting}

Het onderzoek dat heeft geleid tot dit proefschrift had tot doel om bij te dragen aan het begrip van de dynamica van getij en zandbanken in getijdengeulen.

Hoofdstuk 2 beschrijft hoe het verlies van impuls op wadplaten de karakteristieken van het getij in de geul beïnvloedt. Voorbeelden hiervan zijn de tijdstippen van hoog- en laagwater, het verval en de verhouding tussen de maximale stroomsnelheid van de eb en de vloed. Bij opkomend water stroomt er water (met impuls) van de diepe geulen naar de ondiepe platen. Een aantal uur later, bij afgaand tij, stroomt het water weer terug de geulen in. Een groot deel van het impuls in de langsgeulrichting is dan echter verloren gegaan door bodemwrijving op de platen. Om dit te analyseren is een semi-analytisch model ontwikkeld dat de getijbeweging simuleert van een geul die aan beide uiteinden is verbonden met open zee en aan de zijkanten ondiepe platen heeft. De waterbeweging werd geforceerd door een tweemaaldaagsgetij $\left(\mathrm{M}_{2}\right.$-getij) aan beide open uiteinden. Door aan te nemen dat het verval klein is ten opzichte van de waterdiepte en dat de breedte van de geul vergelijkbaar is met die van de platen aan de zijkant, kon de waterbeweging bij benaderding opgelost worden met behulp van een perturbatieve analyse. De resultaten laten zien dat het impulsverlies in de geul leidt tot een afname van de $\mathrm{M}_{2}$-amplitudes van zowel de stroomsnelheid als de waterhoogte en dat de verhouding tussen de maximale vloed- en ebstromingen toeneemt. De afname van de $\mathrm{M}_{2}$-amplitudes komt doordat het water dat de geul instroomt tijdens het afgaand tij nog nauwelijks stroomt in de langsrichting van de geul. Dit remt de stroming in de geul af. Het feit dat de verhouding tussen de maximale eb- en vloedstromingen toeneemt, kan worden verklaard door de term die het impulsverlies beschrijft om te schrijven tot een term die gedurende het afgaand tij advectie van impuls beschrijft. Van de laatste is het bekend dat die de verhouding als dusdanig beïnvloedt. Het gevolg hiervan is dat, wanneer het getij vloeddominant is, de $\mathrm{M}_{4}$-amplitude toeneemt door het impulsverlies op de platen. Het effect van het impulsverlies is groter wanneer de verhouding van de breedte van de platen en 
die van de geul groter is. Het effect is minder gevoelig voor de helling van de platen, de bodemwrijvingscoëfficiënt, de lengte van de geul en het faseverschil tussen de twee inkomende getijgolven aan de open uiteinden.

Het onderzoek naar het impulsverlies op wadplaten in hoofdstuk 2 wordt in hoofdstuk 3 gevolgd door een studie naar de initiële vorming van getijdenzandbanken in geulen. In de literatuur was er tot nog toe inconsistentie over de mogelijke afhankelijkheid van de zandbankgolflengte en de geulbreedte. Observaties en numerieke modellen toonden deze afhankelijkheid wel aan, maar Leuven et al. (2016) lieten zien dat de semi-analytische modellen van Schramkowski et al. (2002) en Seminara and Tubino (2001), die bedoeld waren om de initiële vorming van zandbanken te verklaren, deze afhankelijkheid niet kon reproduceren. Het doel van hoofdstuk 3 was daarom om het model van Schramkowski et al. (2002) zodanig uit te breiden dat de zandbankgolflengte van het snelst groeiende patroon (de voorkeursgolflengte) afhangt van de geulbreedte en nog steeds patronen reproduceert die grofweg overeenkomen met natuurlijke (waargenomen) zandbankpatronen.

De belangrijkste uitbreiding van het model van Schramkowski et al. (2002) betreft het meenemen van de effecten van horizontale turbulente uitwisseling van sediment en in het bijzonder het resulterende bodemhellingseffect. De reden dat het uitgebreide model, in tegenstelling tot het model van Schramkowski et al. (2002), een duidelijke afhankelijkheid tussen de zandbankgolflengte en de geulbreedte laat zien, is als volgt. Ten eerste heeft het patroon met de grootste groeisnelheid (het voorkeurspatroon) in bredere geulen meer banken in de laterale richting. Dat wil zeggen dat dit patroon een hoger preferent aantal dwarsoscillaties heeft. Ten tweede neemt, in het interval van geulbreedtes waarvoor het preferente aantal dwarsoscillaties constant is, de voorkeursgolflengte toe met toenemende breedte. Wanneer het preferente aantal dwarsoscillaties echter verandert (met 1 toeneemt), neemt de voorkeursgolflengte weer af. In het model van Schramkowski et al. (2002) is het interval van breedtes met een constant preferent aantal dwarsoscillaties relatief klein. Dat wil zeggen dat met toenemende breedte, voordat de voorkeursgolflengte significant kan toenemen, het preferente aantal dwarsoscillaties stijgt en daarmee de voorkeursgolflengte weer afneemt. Het gevolg hiervan is dat de voorkeursgolflengte vrijwel gelijk blijft bij een toenemende geulbreedte. In het huidige model is de horizontale turbulente uitwisseling van sediment toegevoegd met als gevolg een sterker bodemhellingseffect. Aangezien bodemhellingseffecten de groeisnelheden van patronen met sterke gradiënten verlagen, heeft het voorkeurspatroon in het huidige model een lager preferent aantal dwarsoscillaties. Dit betekent ook dat het preferente aantal dwarsoscillaties constant blijft over een groter interval van geulbreedtes dan in het model van Schramkowski et al. (2002). De breedteafhankelijkheid volgt nu uit het feit dat in dit interval de voorkeursgolflengte toeneemt met toenemende breedte en dat de voorkeursgolflengte bovendien sterker toeneemt voor lagere preferent aantal dwarsoscillaties.

Een gevoeligheidsanalyse bevestigt de bevindingen van eerdere studies die 
lieten zien dat de zandbankgolflengte afhangt van de stroomsnelheid, de waterdiepte en de geulbreedte. Verder laat een benadering voor de voorkeursgolflengte in termen van drie interne lengteschalen (geulbreedte, getijexcursielengte, wrijvingslengteschaal) zien dat wanneer een van deze lengteschalen klein is, de gevoeligheid voor de desbetreffende lengteschaal groot is (en andersom) vergeleken met de andere twee lengteschalen.

Naast het effect van horizontale turbulente uitwisseling van sediment was ook de rol van het Corioliseffect op de initiële vorming van zandbanken tot nog toe nog niet bestudeerd. Zandbanken zijn echter ook te vinden in systemen waar het Rossbygetal dichtbij één ligt. Dit impliceert dat het Corioliseffect niet verwaarloosd kan worden. Bovendien lieten numerieke modellen zien dat de initiële vorming van zandbanken gevoelig is voor de waarde van de Coriolisparameter. Het doel van hoofdstuk 4 was daarom de rol van het Corioliseffect op de initiële vorming van zandbanken in een getijdengeul zichtbaar te maken. Met behulp van de inzichten verkregen in hoofdstuk 3, is een nieuw model ontwikkeld waar het Corioliseffect in meegenomen wordt. Het belangrijkste inzicht dat hieruit naar voren komt, is dat het mechanisme dat verantwoordelijk is voor het ontstaan van cyclonaal gedraaide zandbanken op de continentale plaat ook aanwezig is in getijdengeulen. Wanneer het inverse Rossbygetal groter is dan ongeveer een half, zorgt dit ervoor dat het snelst groeiende patroon een combinatie is van de zandbankpatronen uit hoofdstuk 3 en cyclonaal gedraaide zandbanken. Ten slotte verhoogt het Corioliseffect de initiële groeisnelheid zonder de voorkeursgolflengte significant te beïnvloeden.

Volgend op de twee studies over de initiële vorming van getijdenzandbanken behandelt hoofdstuk 5 de langetermijndynamica van de zandbanken. Deze langetermijndynamica is eerder bestudeerd in een laboratorium en in numerieke modellen. De meeste van de numerieke modellen waren zogenaamde globale modellen en simuleerden een volledige geul van de zee tot aan een gesloten uiteinde (of rivier). In tegenstelling tot globale modellen, beschrijven lokale modellen alleen een kort stukje van de geul. Dit maakt een diepere analyse mogelijk. In slechts één van de eerdere studies over dit specifieke onderwerp met numerieke modellen was sprake van een dergelijk lokaal model. Dit model beperkte zich echter tot (vaak onrealistisch) kleine waarden van de wrijvingsparameter. Het doel van hoofdstuk 5 was om de langetermijndynamica van zandbanken in een getijdengeul in realistische parameterregimes bloot te leggen. In het bijzonder werd bekeken of niet-triviale morfodynamische evenwichten bestonden, werd de langetermijndynamica vergeleken met de initiële dynamica en de gevoeligheid voor geulbreedte en de Coriolisparameter bestudeerd. Hiervoor is een nieuw lokaal numeriek model ontwikkeld. Dit model heeft tot doel een brug te slaan tussen de modellen die de initiële vorming bestuderen, het lokale numerieke model met kleine wrijving en de globale numerieke modellen die de langetermijndynamica bestuderen. Het modeldomein is periodiek in de langsgeulrichting en representeert een deel van een getijdengeul die ongeveer 3 meter diep en 1-2 kilometer breed is en stromingen vertoont van ongeveer 
$0.5 \mathrm{~m} / \mathrm{s}$ (grofweg representatief voor het Exe estuarium).

Vier tijdsintegratiesimulaties laten zien dat voor de gekozen parameterwaarden (inclusief een realistische bodemwrijvingsparameter) de bodemhoogte na verloop van tijd zo goed als ophoudt te veranderen. Deze uiteindelijke patronen vertonen overeenkomsten met de resultaten van globale numerieke modellen, observaties in de natuur en de banken in het laboratorium. In het bijzonder groeien de gemodelleerde zandbanken over het midden van de geul. In de vier tijdsintegratiesimulaties heeft het Corioliseffect maar geringe invloed op deze langetermijnspatronen, maar de manier waarop het patroon tot deze uiteindelijke patronen komt is wel sterk beïnvloed door het Corioliseffect. In sommige gevallen - in het bijzonder in een geul van 1 kilometer breedte - komen de patronen die initieel vormen, zoals voorspeld door de lineaire stabiliteitsanalyses in de voorgaande hoofdstukken, overeen met langetermijnspatronen. In andere gevallen - in het bijzonder in een geul van 2 kilometer breedte - is dit niet het geval. Verder laten de resultaten zien dat het bestuderen van morfodynamische evenwichten met behulp van tijdsintegratoren problematisch is: het is onduidelijk of en wanneer een patroon is geconvergeerd naar een stabiel evenwicht. Als alternatief is hiertoe een nulpuntzoeker gebruikt om morfodynamische evenwichten te vinden voor verschillende geulbreedtes. Voor toenemende breedtes kruisen de banken in toenemende mate het midden van de geul en beweegt de maximumhoogte van de bank weg van de rand van de geul. Deze voorlopige resultaten moeten worden beschouwd als proof-of-concept om de langetermijndynamica van zandbanken systematisch verder te onderzoeken. 


\section{Acknowledgements}

I would like to first thank my two supervisors: Huib and Henk. Huib, thank you for all your help and for safeguarding the progress of this thesis. I greatly appreciate your personal style of supervision. Your teaching inspired me and besides our regular meetings, I enjoyed our discussions about 'hobby questions' concerning the Wadden Sea. Henk, thank you for being the mathematician in my team. Your enthusiasm is contagious and it was great to notice we enjoyed the same type of methodologies and discussions. I've always felt supported by you and I am very grateful for the numerous times you helped me with questions I had about the methods we are using. The thursday mornings in 'de Ontdekking' were always meetings I looked forward to.

IMAU was a great place to work. Floor, Clara and Marcel thank you for all the support. Bo, Bing, Wei and Wim, thank you for welcoming me in the coastal group. Bo and Getachew, it was a pleasure to share my initial office with you. Bo, you were a big help in settling in. Bing, thank you for the ping-pong games and the initial help with the morfo-code. George, thank you for your interest in my work. I enjoyed working together in Delft and Utrecht. Steffie, Youri and Qing-yi thank you for the coffee breaks in Delft. Eltjo, thank you for the coffee breaks at J and G. Albert Falqués and Daniel Calvete thank you for your hospitality at UPC in Barcelona and Roland Garnier thank you for introducing me to the morfo model. Theo, thanks a lot for the nice discussions and the NIOZ trip.

Abdel, Jinyang and Janneke, it was great to be in the coastal group together. Abdel, thank you for all the (overly sugary) tea and coffee. Jinyang, I greatly enjoyed our discussions, ping-pong games and sharing the office with you was a pleasure. Janneke, I am very grateful for all the Skype talks we had during the pandemic. Besides the coastal group many others contributed to my time at IMAU. Leo, thank you for our discussions. Erik van Sebille, thank you for your friendly enthusiasm. Peter, thank you for the conversations about flat bottomed boats. Philippe, thank you for the discussions about Python. David, 
your constant change is fun. Martina, thank you for the rice plant supply for the office. André, thank you for all your physics knowledge. Malika, speed iceskating, sailing and morning plonses were great. Verena, thank you for all the swims, talks and dinners. Clara, thank you for your point of views. Daniele, thank you for all the serious and bullshit talks and for showing us around in Italy. Erik, thank you for joining me buitengaats, for the discussions about scientific computing and for bringing a bit of Groningen to IMAU. Annerose, thank you for all your Dutch. Simone, thank you for the great pictures. Oscar, thank you for convincing me to learn Julia. Maxim, thank you for all nice talks on data-driven methods. Laura, thank you for being the perfect roommate, this was especially valuable when working from home. Lastly, I would like to thank my parents, Marjan en Bouke, and my sister, Wytske, for all their support in the last 29 years! 


\section{Curriculum vitae}

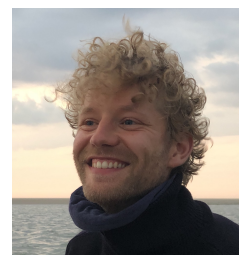

Tjebbe Hepkema was born in Eelde on the 21st of October, 1991. In 1992, his parents introduced him to the tidal channels in the Wadden Sea. In 2013 he obtained his bachelor's degree in mathematics from Utrecht University, where he wrote his bachelor thesis under the supervision of André Henriques. In 2016 he obtained his master's degree in mathematics at the same university. The topic of his master thesis was nonlinear tides in a tidal channel and was written under the supervision of Antonios Zagaris and Huib de Swart. Afterwards, he continued to work with Huib de Swart and co-promotor Henk Schuttelaars to write the current $\mathrm{PhD}$ thesis. 